\title{
Molecular evolution and \\ functional characterisation of \\ tunicate xenobiotic receptors
}

BY

INGRID RICHTER

A thesis

submitted to the Victoria University of Wellington

in fulfilment of the requirements for the degree of

Doctor of Philosophy
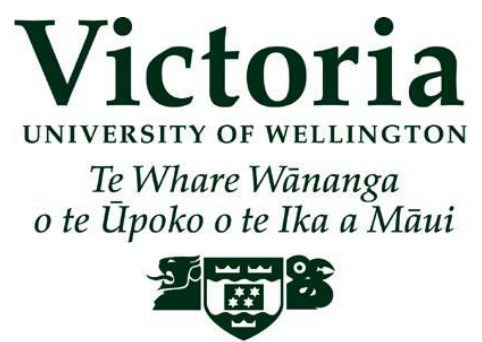

www.vuw,ac.nz

2015 


\section{Abstract}

Marine microorganisms generate a wide range of 'bioactive' compounds that can have far-reaching effects on biological and ecological processes. Metazoans have developed specialised biochemical pathways that metabolise and eliminate potentially toxic chemicals (xenobiotics) from their bodies. The vertebrate xenobiotic receptor, pregnane $X$ receptor (PXR), is a ligand-activated nuclear receptor transcription factor regulating expression of multiple detoxification genes. Ligand-binding domains (LBDs) of vertebrate PXR orthologues may have adaptively evolved to bind toxins typically encountered by these organisms. Marine invertebrate filter-feeders are exposed to relatively high concentrations of xenobiotics associated with their diet. Tunicates (phylum: Chordata) are of particular interest as they form the sister clade to the Vertebrata. Genomes of the solitary tunicate Ciona intestinalis and the colonial tunicate Botryllus schlosseri both encode at least two xenobiotic receptors that are orthologues to both the vertebrate vitamin D receptor (VDR) and PXR.

Pursuing the idea that tunicate xenobiotic receptors (VDR/PXR) may adaptively evolve to bind toxic chemicals commonly present in an organism's environment, this thesis aims to identify if: (i) adaptive evolution is acting on putative tunicate VDR/PXR orthologues to enhance binding of dietary xenobiotics; (ii) these receptors are activated by dietary xenobiotics (e.g. microalgal biotoxins) and; (iii) tunicate VDR/PXR LBDs can be used as sensor elements in yeast bioassays for the detection of both natural and synthetic bioactive compounds. To identify genetic variation and to search for evidence of positive selection, next-generation sequencing was performed on three tunicate VDR/PXR orthologues genes. Recombinant yeast (Saccharomyces cerevisiae) cell lines were developed for the functional characterisation of tunicate VDR/PXR LBDs. These tunicate VDR/PXR LBD-based yeast bioassays were utilised to detect known microalgal biotoxins, natural bioactive compounds, and environmental contaminants.

Next-generation sequencing revealed both an unusually high genetic diversity and strong purifying selection in VDR/PXR orthologues from $C$. intestinalis and 
B. schlosseri. Single-base-deletion allelic variants were found in $C$. intestinalis VDR/PXR orthologues resulting in predicted proteins having a DNA-binding domain but lacking a LBD. The persistence of these variants may reflect constitutive expression of detoxification genes as a selective advantage in the marine environment.

To assess the functional characteristics of tunicate VDR/PXR orthologues, recombinant yeast cell lines were developed that express VDR/PXR $\alpha B D s$ from $C$. intestinalis and $B$. schlosseri. These chimeric proteins mediate liganddependent expression of a lac $Z$ reporter gene which encodes an easily assayed enzyme ( $\beta$-galactosidase). These yeast bioassays were highly sensitive towards both synthetic and natural toxins (coefficients of variance, CV $<25 \%$ ). Microalgal biotoxins (okadaic acid and portimine) were two orders of magnitude more potent than synthetic chemicals, which was consistent with the hypothesis that tunicate xenobiotic receptors can bind marine bioactive compounds frequently present in a filter-feeder's diet.

Following these functional studies, the yeast bioassays were tested in a more applied context by screening the following compounds: (i) natural bioactive compounds that represent promising compounds for drug development and; (ii) synthetic chemicals that are common environmental pollutants. Of the 34 compounds tested, 30 were active in the tunicate yeast bioassays. The yeast bioassays were particularly sensitive towards a small number $(n=11)$ of marine and terrestrial bioactive compounds (CJ-13-014, CJ-13-104, thysanone and naringin) and emerging contaminants such as pharmaceuticals (ketoconazole), antifungals (radicicol), preservatives (butylated hydroxtoluene) and surfactants (oil dispersants), generating CV values $<25 \%$. Activities of the remaining 19 compounds were highly variable and appeared to depend on several factors, such as solvent used, duration of exposure and type of recombinant protein expressed (e.g. C. intestinalis versus B. schlosseri VDR/PXRa).

In conclusion, the yeast bioassay developed in this thesis, with further development, may provide a template for novel bioassays that may find application in routine microalgal biotoxin testing and environmental monitoring. These bioassays may also assist in the identification of marine bioactive compounds as drug lead compounds. 


\section{Table of Contents}

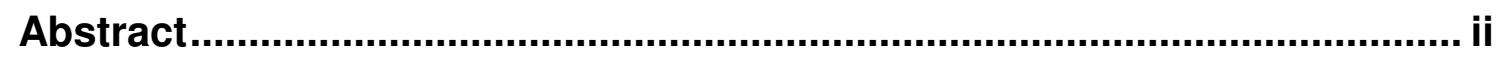

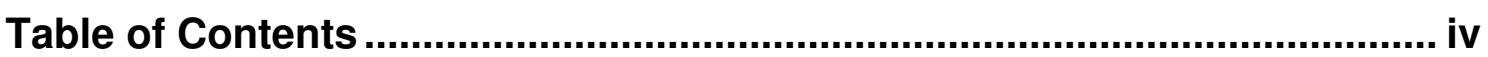

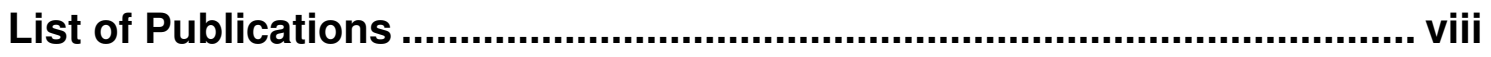

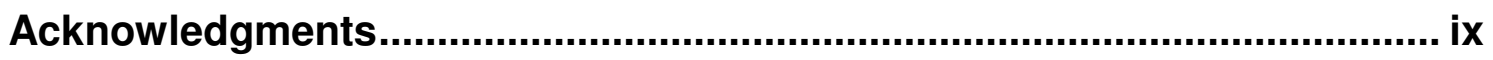

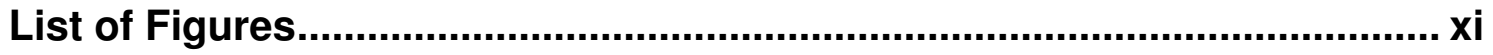

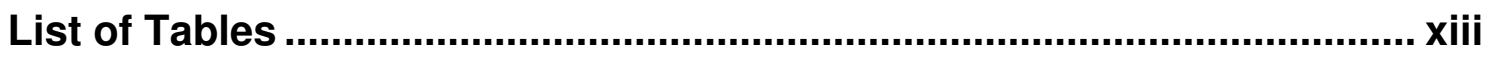

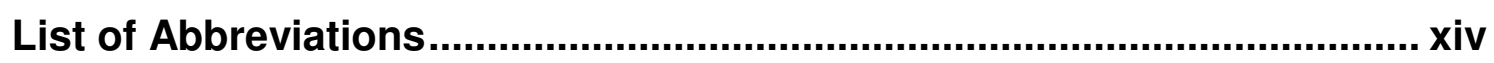

List of Appendices ............................................................................. xviii

CHAPTER ONE: Molecular evolution and functional characterisation of tunicate xenobiotic receptors and their use as sensor elements in yeast bioassays for bioactive compounds: an overview ..................................... 21

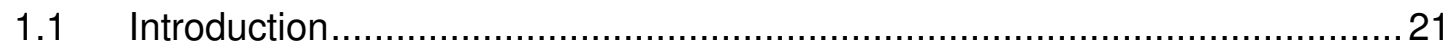

1.1.1 Dietary bioactive chemicals .......................................................22

1.1.2 Detoxification pathways and their transcription level regulation .............25

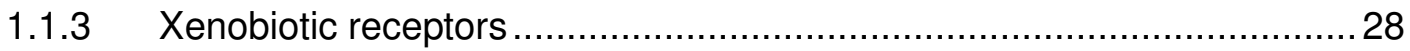

1.1.4 Suitable expression systems for functional studies of nuclear receptors 32

1.2 Thesis structure and research hypotheses ………......................................... 39

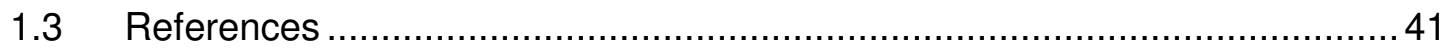

PART ONE: Detection of adaptive evolution in tunicate xenobiotic receptors using molecular tools

CHAPTER TWO: Transcripts of tunicate orthologues of the pregnane $X$ receptor: characterisation and natural variation ........................................ 55

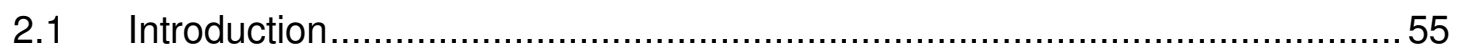

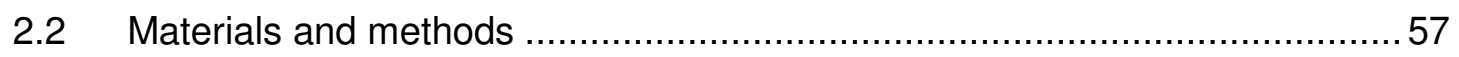

2.2.1 Tunicate collection and tissue sampling ...........................................57

2.2.2 Amplification and Sanger sequencing of cDNA sequences....................58

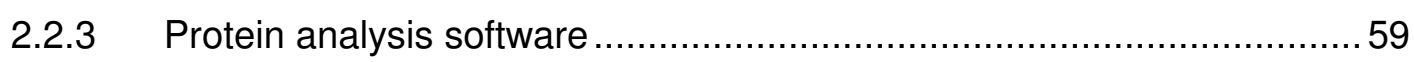

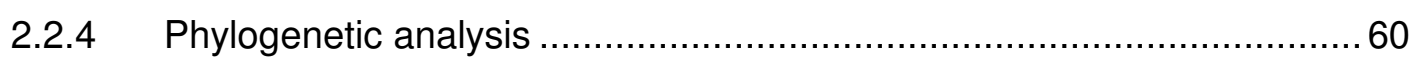


2.2.5 Detection of Ciona intestinalis VDR/PXR $\alpha$ and $\beta$ gene transcripts .........60 60

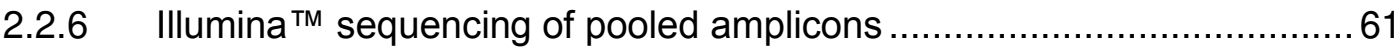

2.2.7 Identification and analysis of polymorphisms in pooled amplicon

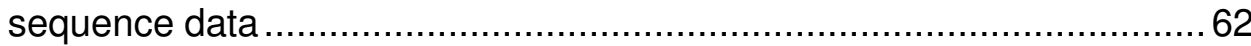

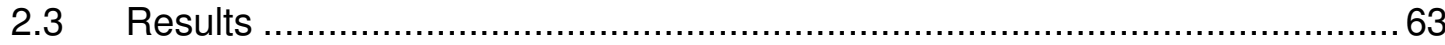

2.3.1 Determination of tunicate VDR/PXR $\alpha$ and $\beta$ partial coding sequences .. 63

2.3.2 Detection of VDR/PXR $\alpha$ and $\beta$ transcripts in the Ciona intestinalis digestive tract ............................................................................... 69

2.3.3 Sequence polymorphisms detected in tunicate VDR/PXR amplicons ....71

2.3.4 Frameshift polymorphisms in the Ciona intestinalis VDR/PXR coding sequences

2.3.5 Haplotype diversity in two Botryllus schlosseri populations from different geographical locations ................................................................... 74

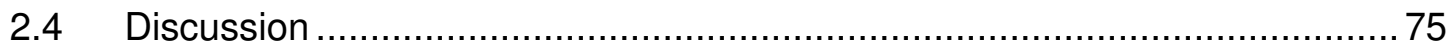

2.4.1 Determination of tunicate VDR/PXR $\alpha$ and $\beta$ sequences ......................75

2.4.2 Expression of VDR/PXR $\alpha$ and $\beta$ transcripts in the Ciona intestinalis

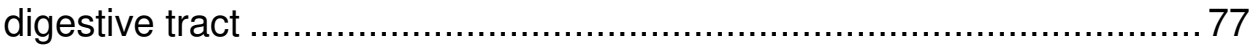

2.4.3 Variation in the tunicate VDR/PXR $\alpha$ and $\beta$ sequences .........................77

2.4.4 Frameshift polymorphisms...................................................... 78

2.4.5 Haplotype diversity in two Botryllus schlosseri populations from different geographical locations ................................................................ 79

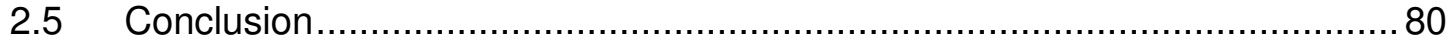

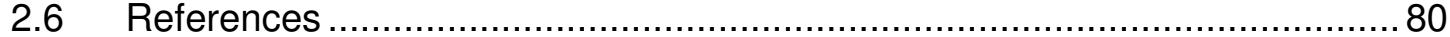

PART TWO: Functional characterisation of tunicate xenobiotic receptors and their application in yeast-based bioassays ......................................... 88

\section{CHAPTER THREE: Detection of marine microalgal biotoxins using} bioassays based on functional expression of tunicate xenobiotic

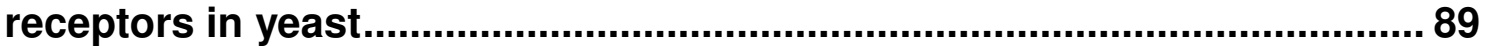

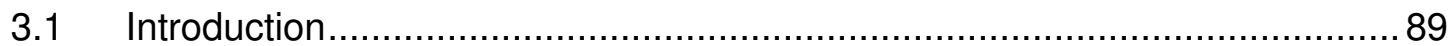

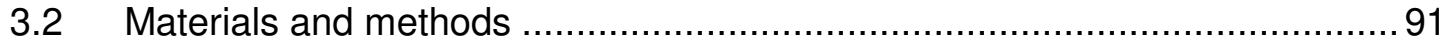

3.2.1 Compounds tested in the yeast bioassay........................................... 91

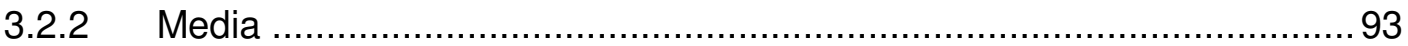

3.2.3 Generation of plasmid constructs.................................................... 93

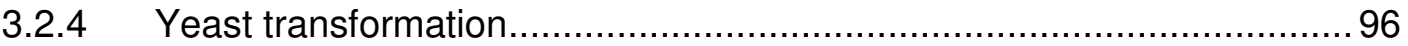

3.2.5 Preparation of yeast starter stocks.................................................... 96 


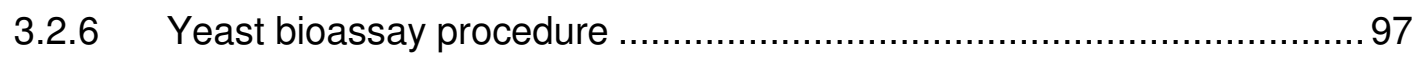

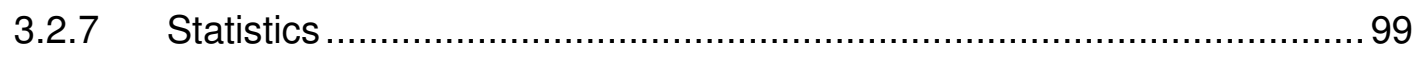

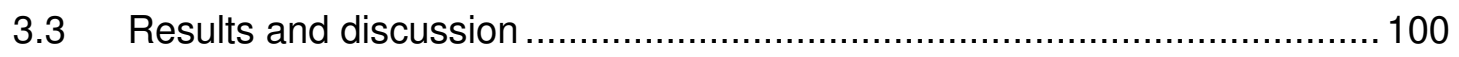

3.3.1 Development of the bioassay yeast strains ..................................... 100

3.3.2 Yeast bioassay media optimisation............................................... 102

3.3.3 Activity of synthetic toxicants in the yeast bioassays......................... 107

3.3.4 Activity of natural microalgal biotoxins in the yeast bioassays.............111

3.3.5 Characteristics of the yeast bioassays ...................................... 115

3.4 Conclusion................................................................................ 118

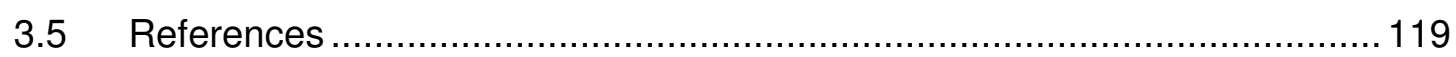

\section{CHAPTER FOUR: Utilising tunicate xenobiotic receptors in yeast}

bioassays for high-throughput screening of bioactive compounds ....... 125

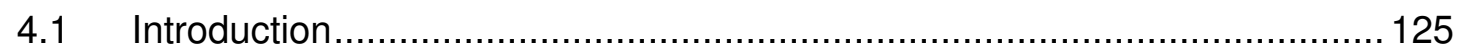

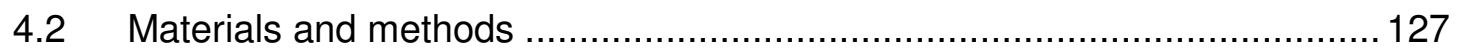

4.2.1 Compounds tested in the yeast bioassay ........................................ 127

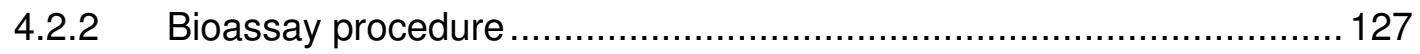

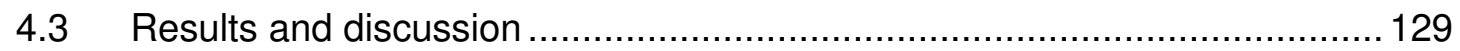

4.3.1 Activity of microalgal biotoxins in the yeast bioassays ........................ 132

4.3.2 Activity of fungal metabolites in the yeast bioassays .......................... 135

4.3.3 Activity of plant-derived bioactive compounds in the yeast bioassays.. 140

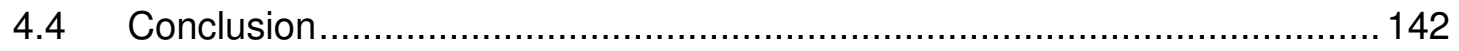

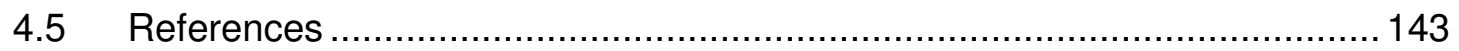

\section{CHAPTER FIVE: Utilising tunicate xenobiotic receptors in yeast bioassays} for detection of environmental pollutants............................................. 149

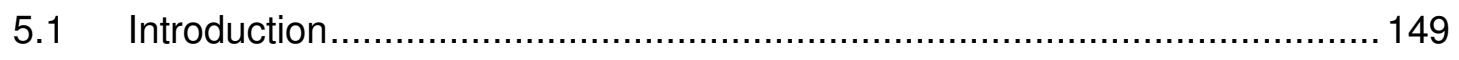

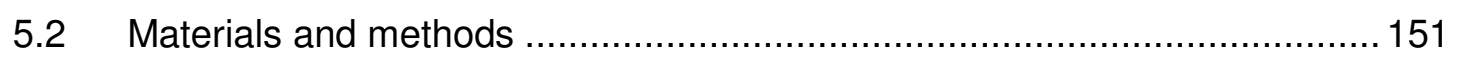

5.2.1 Compounds tested in the yeast bioassay........................................ 151

5.2.2 Yeast bioassay procedure ....................................................... 152

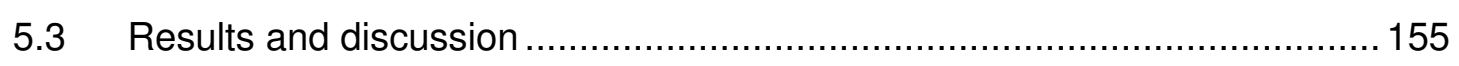

5.3.1 Activity of synthetic chemicals in the tunicate yeast bioassays ............ 155

5.3.2 Activity of commercial oil dispersants in the tunicate yeast bioassays .171

5.3.3 Characteristics of the yeast bioassays .......................................... 174

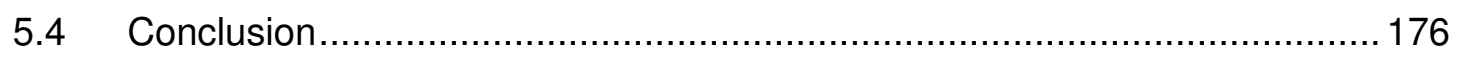

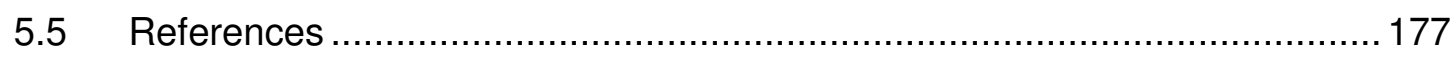


CHAPTER SIX: Conclusions..................................................................... 187

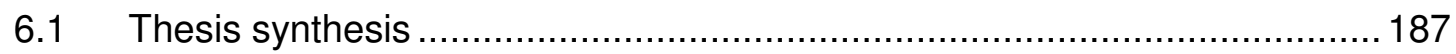

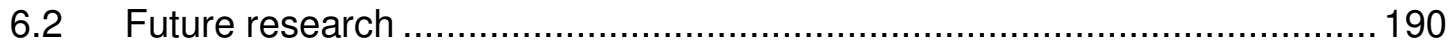

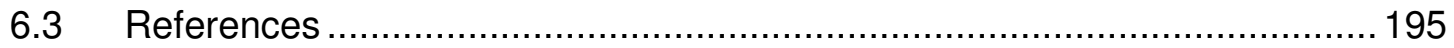




\section{List of Publications}

This thesis is composed of a series of independent manuscripts. For all manuscripts, I conceived the questions/experiments in consultation with my thesis supervisor, conducted the data analyses and drafted each manuscript. My primary thesis supervisor Dr Andrew Fidler (Cawthron Institute) reviewed each draft manuscript and gave advice, and some assistance, with experimental and laboratory work. I collected all field samples and conducted the laboratory work, unless otherwise stated.

Chapter One has been published as:

Richter, I \& Fidler AE (2014) Marine invertebrate xenobiotic-activated nuclear receptors: their application as sensor elements in high-throughput bioassays for marine bioactive compounds. Marine Drugs. 12(11): 5590-618. doi:

10.3390/md12115590. IF: 3.512

Chapter Two has been published as:

Richter, I \& Fidler AE (2015) Transcripts of tunicate orthologues of the pregnane $X$ receptor $(P X R)$ : characterisation and natural variation. Marine Genomics. doi: 10.1016/j.margen.2015.05.006. IF: 1.971

Chapter Three has been published as:

Richter, I \& Fidler AE (2015) Detection of marine microalgal biotoxins using bioassays based on functional expression of tunicate xenobiotic receptors in yeast. Toxicon 95(0): 13-22. doi: 10.1016/j.toxicon.2014.12.011. IF: 2.381

Chapter Four is being prepared as a manuscript for submission with the proposed title:

Richter, I \& Fidler AE. Novel yeast bioassays based on tunicate xenobiotic receptors for high-throughput screening of natural bioactive compounds. Sensors. IF: 2.048

Chapter Five represents a possible future publication. 


\section{Acknowledgments}

First and foremost I would like to thank my supervisors Dr Andrew Fidler from the Cawthron Institute (New Zealand) and Prof Ken McNatty from Victoria University of Wellington (New Zealand) for their guidance and contribution to this thesis. This research was funded by the New Zealand Ministry of Business, Innovation and Employment (Contract No. CAWX1001).

I received invaluable assistance from numerous people for the collection of field samples. Prof Baruch Rinkevich (National Institute of Oceanography, Israel) and Dr Andrew Fidler (Cawthron Institute) sampled Botryllus schlosseri colonies in Israel. I would also like to thank Dan McCall (Shellfish Production and Technology, New Zealand) for allowing me to tag along on fieldtrips to Pelorus Sound, and Dr Chris Woods (National Institute of Water and Atmospheric Research, New Zealand) for collecting samples during his dive surveys in the Christchurch harbour (Lyttelton, New Zealand).

Numerous people provided help and assistance with this thesis in many different ways. Dr Jonathan Banks and Dr Kirsty Smith (both Cawthron Institute) critically reviewed the manuscript published in 'Marine Drugs' (Chapter One).

Dr Ayelet Voskoboynik (Stanford University, U.S.A.) kindly provided access to unpublished B. schlosseri genomic sequence data. Prof Baruch Rinkevich (National Institute of Oceanography) shared his knowledge about B. schlosseri. Rod Asher (Cawthron Institute) provided assistance with dissections and gave advice on Ciona morphology (Chapter Two). Andy Selwood and Dr Lincoln MacKenzie (both Cawthron Institute) provided microalgal toxin standards (Chapter Three). Amanda Heapy and Prof Margaret Brimble (both University of Auckland, New Zealand) provided natural bioactive chemicals (Chapter Four). I would like to thank Alexis Simon (École Normale Supérieure, France) for laboratory assistance (Chapter Five). 
I would also like to extend my sincere gratitude to Dr Susie Wood, Cherie Johansson and Dr Jonathan Puddick (all Cawthron Institute) for their support and critical review of this thesis.

Last, but certainly not least, the biggest thank you goes to my parents, family and friends who have always been an enormous support to me. A special thanks to Josh for his love, support and encouragement.

"A lot of people have gone further than they thought they could because someone else thought they could."

Richard Branson 


\section{List of Figures}

Figure 1.1 Examples of filter-feeding and surface-grazing marine invertebrates.

Figure 1.2 Schematic overview of metazoan detoxification pathways.

Figure 1.3 Schematic structure of nuclear receptors.

Figure 1.4 Simplified schematic of modified yeast one-hybrid bioassays.

Figure 2.1 Genetic diversity within the primary structures of the predicted tunicate xenobiotic receptor proteins.

Figure 2.2 Molecular phylogeny of nuclear receptors including the three tunicate xenobiotic receptors investigated in this study.

Figure 2.3 Schematic summary of gene transcripts in gut regions of Ciona intestinalis including two xenobiotic receptors investigated in this study.

Figure 3.1 Structures of four synthetic chemicals and five algal toxins.

Figure 3.2 Schematic of fusion genes/chimeric proteins generated in this study.

Figure 3.3 Example of a 96-well plate format used in the tunicate yeast bioassays.

Figure 3.4 Media concentrations of L-histidine influence the growth rate of three recombinant yeast strains.

Figure 3.5 Influence of L-histidine concentrations on background activity of $\beta$-galactosidase.

Figure 3.6 Activities of four synthetic chemicals tested in the yeast bioassays.

Figure 3.7 Activities of three microalgal biotoxins tested in the yeast bioassays.

Figure 4.1 Structures of 16 natural products and relevant analogues. 
Figure 4.2 Activities of gymnodimine and related analogues tested in the yeast bioassays.

Figure 4.3 Activities of fungal metabolites tested in the yeast bioassays.

Figure 4.4 Activity of naringin tested in the yeast bioassays.

Figure 5.1 Structures of 13 emerging contaminants tested in the yeast bioassays.

Figure 5.2 Activities of synthetic preservatives tested in the yeast bioassays.

Figure 5.3 Activities of endocrine disrupting chemicals tested in the 164 yeast bioassays.

Figure 5.4 Activities of fungicides tested in the yeast bioassays. 167

Figure 5.5 Activities of $n, n$-diethyl- $m$-toluamide (DEET) and 170 diclofenac sodium salt tested in the yeast bioassays.

Figure 5.6 Activities of oil dispersants tested in the yeast bioassays. 173 


\section{List of Tables}

Table 2.1 Sequence identities of xenobiotic receptor orthologues. 56

Table 2.2 Summary statistics for polymorphisms detected in tunicate xenobiotic receptor orthologues.

Table 3.1 Activation of the tunicate yeast bioassays by four synthetic chemicals.

Table 3.2 Activation of the tunicate yeast bioassays by five algal toxins.

Table 3.3 Inter-plate variability of the tunicate yeast bioassays.

Table 4.1 Activation of the tunicate yeast bioassays by 16 natural products and relevant analogues.

Table 5.1 Activation of the tunicate yeast bioassays by 13 emerging contaminants.

Table 5.2 Activation of the tunicate yeast bioassays by five commercial oil dispersants. 


\section{List of Abbreviations}

AD

$\mathrm{ADH} 1$

AF-2

AhR

ANOVA

Akt

$\mathrm{BaP}$

bp

BLAST

BPA

$B s \vee D R / P X R \alpha$

CAR

$95 \% \mathrm{Cl}$

CNDR/PXRa

CNDR/PXR $\beta$

cos-7

CPRG

CV

CYP

DBD
Activation domain

alcohol dehydrogenase 1

Activation function domain 2

Aryl hydrocarbon receptor

One-way analysis of variance

Protein kinase B

Benzo[a]pyrene

Base pair

Basic Local Alignment Search Tool

Bisphenol-A

Botryllus schlosseri VDR/PXR orthologue $\alpha$

Constitutive androstane receptor

$95 \%$ confidence interval

Ciona intestinalis VDR/PXR orthologue $\alpha$

Ciona intestinalis VDR/PXR orthologue $\beta$

Fibroblast-like cell line derived from monkey kidney tissue

Chlorophenol red- $\beta$-D-galactopyranoside

Coefficient of variance

Cytochrome P450 enzyme

DNA-binding domain 
$d_{N} / d_{S}$

$\mathrm{D}_{\mathrm{T}}$

EC

$\mathrm{EC}_{50}$

EDC

ER

ERE

EST

FRET

GAL4

GAL4-DBD

GM

GR

GST

HeLa

HepG2

HRV

HSP90

HTS

$\mathrm{HuH} 7$

$\mathrm{kb}$

LB
Ratio of non-synonymous substitutions per non-

synonymous site $\left(d_{N}\right)$ to synonymous substitutions per synonymous site $\left(d_{S}\right)$

Tajima's D

Emerging contaminants

Mean effective concentration

Endocrine disrupting chemical

Estrogen receptor

Estrogen receptor response element

Expressed sequence tag

Fluorescence resonance energy transfer

Yeast DNA-binding transcription factor

GAL4 DNA-binding domain

Growth media

Glucocorticoid receptor

Glutathione S-transferase

Human cervical cancer-derived cell line

Human liver carcinoma-derived cell line

Human rhinovirus

Heat shock protein 90

High-throughput screening

Human hepatocyte carcinoma-derived cell line

Kilo base

Luria broth 
LBD

MCF-7

MCS

MM

MRP

NCBI CD

NR

NR1I

NR1J

$\pi$

PAHs

PCN

PCR

Pfam

PTX-2

PTX-11

PXR

qPCR

QSAR

$\mathrm{RXR}$

SAM

SMART

SNP
Ligand-binding domain

Human breast cancer-derived cell line

Multiple cloning site

Minimal media

Multi-drug resistance-associated protein

National Center for Biotechnology Information Conserved

Domain database

Nuclear receptor

Nuclear receptor sub-family 1 , class I

Nuclear receptor sub-family 1 , class $\mathrm{J}$

Nucleotide diversity

Polycyclic aromatic hydrocarbons

Pregnenolone 16a-carbonitrile

Polymerase chain reaction

Protein Families database

Pectenotoxin-2

Pectenotoxin-11

Pregnane $\mathrm{X}$ receptor

Quantitative polymerase chain reaction

Quantitative structure-activity relationship

Retinoid $\mathrm{X}$ receptor

Seeded assay medium

Simple Modular Architecture Research Tool

Single nucleotide polymorphism 
SRC-1

TCPOBOP

TGF- $\beta$

$\mathrm{Ti} / \mathrm{TV}$

UAS

VDR

$\theta_{W}$

VP16

VP16-AD

yEGFP
Steroid receptor co-activator 1

1,4-Bis[2-(3,5-dichloropyridyloxy)]benzene

Growth factor beta super-family

Ratio of transitions (Ti) versus transversions (Tv)

Upstream activation sequence

Vitamin D receptor

Watterson's Theta

Herpes simplex virion protein 16

VP16 activation domain

Yeast-enhanced green fluorescence protein 


\section{List of Appendices}

Appendix One: $\quad$ Primers used for amplification of tunicate xenobiotic receptor orthologues.

201

Appendix Two: $\quad$ Conserved domain predictions in three tunicate xenobiotic receptor protein sequences generated in this study.

Appendix Three: Alignment of predicted DNA-binding domains (DBDs) and ligand-binding domains (LBDs) from pregnane $X$ receptor $(P X R)$-related proteins from vertebrates, invertebrate chordates and protostome taxa.

Appendix Four: Amplification of Ciona intestinalis vitamin D receptor/pregnane $X$ receptor $\alpha(V D R / P X R \alpha)$, VDR/PXR $\beta$ and actin partial coding sequences in five tissues using end-point polymerase chain reaction (PCR).

Appendix Five: $\quad$ Approximate probabilities (p) of Cochran's C Test (A) and Tukey HSD Post Hoc Test (B) for the expression of Ciona intestinalis vitamin D receptor/pregnane $X$ receptor $\alpha$ (CNDR/PXR $\alpha), C$. intestinalis VDR/PXR $\beta$ (CNDR/PXR $\beta$ ) and $C$. intestinalis actin (Ciactin) in various gut tissues.

Appendix Six: $\quad$ Illumina ${ }^{\mathrm{TM}}$ sequencing read mapping statistics for Ciona intestinalis vitamin D receptor/pregnane $X$ receptor a (CNDR/PXRa, $\underline{\mathrm{KC} 561370})$, C. intestinalis VDR/PXR $\beta$

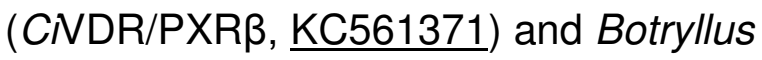
schlosseri VDR/PXRa (BsVDR/PXRa, KC561372). 
Appendix Seven: Coverage by position for Ciona intestinalis vitamin $D$ receptor/pregnane $X$ receptor $\alpha$ (CNDR/PXRa, KC561370), C. intestinalis VDR/PXRß (CNDR/PXRß, KC561371) and Botryllus schlosseri VDR/PXRa (BsVDR/PXRa, KC561372) using a maximum coverage of 40,000 .

Appendix Eight: Nucleotide polymorphisms detected in Ciona intestinalis vitamin $\mathrm{D}$ receptor/pregnane $\mathrm{X}$ receptor a (CNDR/PXRa, 1326 base pairs, bp), $C$. intestinalis VDR/PXR $\beta$ (CNDR/PXR $\beta$, $1569 \mathrm{bp})$ and Botryllus schlosseri VDR/PXRa (BsVDR/PXRa, $1006 \mathrm{bp}$ ) using Illumina ${ }^{\mathrm{TM}}$ sequencing.

Appendix Nine: $\quad$ Estimated transition/transversion rate ratios $(\mathrm{Ti} / \mathrm{TV}$ ) for Ciona intestinalis vitamin $\mathrm{D}$ receptor/pregnane $X$ receptor $\alpha(C N D R / P X R \alpha$, 1326 base pairs, bp), $C$. intestinalis VDR/PXR $\beta$ (CNDR/PXR $\beta, 1569 \mathrm{bp}$ ) and Botryllus schlosseri VDR/PXRa (BsVDR/PXRa, 1006 bp) using the Tamura-Nei model as implemented in the MEGA 5 software package. 214 Appendix Ten: $\quad$ Nucleotide polymorphisms detected in Botryllus schlosseri vitamin D receptor/pregnane $X$ receptor $\alpha$ (BsVDR/PXRa, 1006 base pairs, bp) populations from Nelson Marina (New Zealand) and the eastern Mediterranean Coast (Michmoret Beach, Israel).

Appendix Eleven: Activation of the tunicate yeast bioassays by four synthetic chemicals (A) and five algal toxins (B). 
Appendix Twelve: Summary of the templates, primers and restriction enzymes used to generate the plasmids used in this study.

Appendix Thirteen: Summary of the predicted proteins encoded by the seven plasmids used in this study.

Appendix Fourteen: Suppression of growth of recombinant yeast strains by: (A) $n$-butyl-p-aminobenzoate and (B) carbamazepine.

Appendix Fifteen: Suppression of growth of recombinant yeast strains by: (A) (-)-deoxydihydrokalafungin and (B) (+)-deoxykalafungin.

Appendix Sixteen: Activation of the yeast bioassays by 13 emerging contaminants.

Appendix Seventeen: Suppression of growth of recombinant yeast strains by: (A) propyl-4-hydroxybenzoate, (B) triclosan, (C) benzophenone and (D) $n, n-$ diethyl-m-toluamide (DEET).

Appendix Eighteen: Presentations at conferences and meetings. 231 Appendix Nineteen: Publications in refereed journals. 232 


\section{Molecular evolution and functional characterisation of tunicate xenobiotic receptors and their use as sensor elements in yeast bioassays for bioactive compounds: an overview}

\subsection{Introduction}

Bacterial, fungal and microalgal organisms in marine ecosystems collectively generate a wide range of 'bioactive' compounds that can have far-reaching effects on biological and ecological processes (e.g. reproduction, species interactions, population dynamics) as well as on human health and economies (Hay 2009; Landsberg et al. 2009; Mayer 2009). Metazoans have developed specialised biochemical pathways that metabolise and eliminate potentially toxic chemicals (xenobiotics) from their bodies and therefore minimise any associated deleterious effects ( $\mathrm{Li}$ et al. 2007; Testa et al. 2012; Zanger \& Schwab 2013). Xenobiotic receptors, a specific group of ligand-activated nuclear receptors (NRs), bind a structurally diverse range of xenobiotics and then activate genes involved in ligand detoxification (Nakata et al. 2006; Chai et al. 2013).

From an evolutionary perspective, xenobiotic receptors represent a highlyderived branch of the NR family tree- the root of which lies deep at the base of animal phylogeny (Bertrand et al. 2004). Vertebrate xenobiotic receptor proteins (pregnane $X$ receptor, PXR) are unusual amongst the NRs as they display considerable inter-species sequence variation in the functional domain responsible for ligand-binding (ligand-binding domain, LBD; Moore et al. 2002; Zhang et al. 2004; Krasowski et al. 2005a, b). Such inter-taxa PXR LBD sequence differences may reflect adaptive evolutionary changes which enhance PXR binding of dietary bioactives/toxins typically encountered by an organism 
(Moore et al. 2000; Moore et al. 2002; Krasowski et al. 2005a, b; Krasowski et al. 2011a, b).

Marine invertebrate filter-feeders (e.g. Tunicata, Bivalvia) are exposed to a myriad of dietary xenobiotics at relatively high concentrations (Haberkorn et al. 2011; Echevarria et al. 2012). Tunicates (phylum Chordata, subphylum Urochordata; Satoh et al. 2014) are of particular interest because their genomes encode putative xenobiotic receptor genes that are orthologues to vertebrate PXR (Ekins et al. 2008). Because tunicates accumulate high concentrations of dietary xenobiotics, tunicate xenobiotic receptors may have adaptively evolved to enhance binding of these compounds (Fidler et al. 2012). Tunicates are the closest living relatives of vertebrates as they are members of a sister clade to the Vertebrata (Delsuc et al. 2006; Delsuc et al. 2008). Therefore, they are a promising group to target for the development of bioassays that could be used to detect bioactive chemicals that affect vertebrate physiology.

The aims of this thesis were to: (i) determine if adaptive evolution is occurring in three tunicate putative xenobiotic receptor genes; (ii) characterise the function of two tunicate putative xenobiotic receptors using a yeast-based expression system and; (iii) develop highly generic recombinant yeast bioassays using tunicate xenobiotic receptors as sensor elements, with the goal of using these for bioprospecting and to detect harmful environmental contaminants.

\subsubsection{Dietary bioactive chemicals}

Chemicals in animal diets are often viewed as simply energy sources (e.g. carbohydrates, lipids), building blocks (e.g. proteins) or biochemical pathway intermediates (e.g. vitamins). However, it is apparent that some dietary chemicals can alter animal biochemistry and physiology with biological effects ranging from influences on reproduction and development through to acute poisoning (Targett \& Arnold 2001; Raubenheimer \& Simpson 2009; Forbey et al. 2013; Ortiz-Ramirez et al. 2013). Some animal taxa exposed to bioactive dietary xenobiotics have evolved both behavioural and physiological traits to minimise any associated deleterious effects (Dearing et al. 2005). Many animals 
simply avoid eating plants/prey likely to contain toxins with these avoidance behaviours being both instinctual and learnt (Marsh et al. 2006). For example, avoidance of toxic/unpalatable prey by coral reef fish is well-documented (Long \& Hay 2006; Miller \& Pawlik 2013). Bivalve molluscs can limit their exposure to toxic compounds using behavioural responses such as shell closure and restriction of filtration rate (Hegaret et al. 2007; Haberkorn et al. 2011). Despite such avoidance behaviours, the diet of many marine organisms, especially those of filter-feeding and surface-grazing invertebrates (Figure 1.1) will inevitably contain toxic bioactive chemicals that need to be metabolised and eliminated from their bodies (Glendinning 2007; Fernandez-Reiriz et al. 2008; Manfrin et al. 2012; Sotka \& Gantz 2013). 


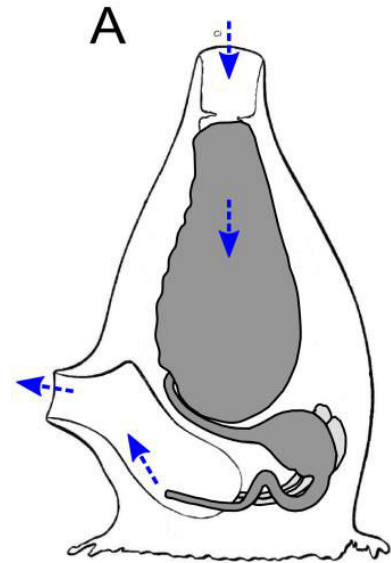

B

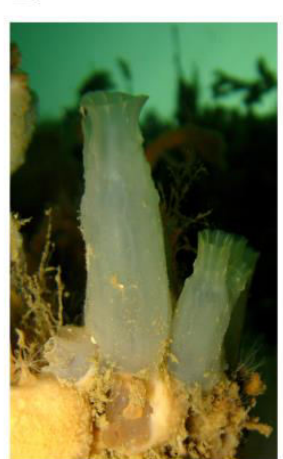

C

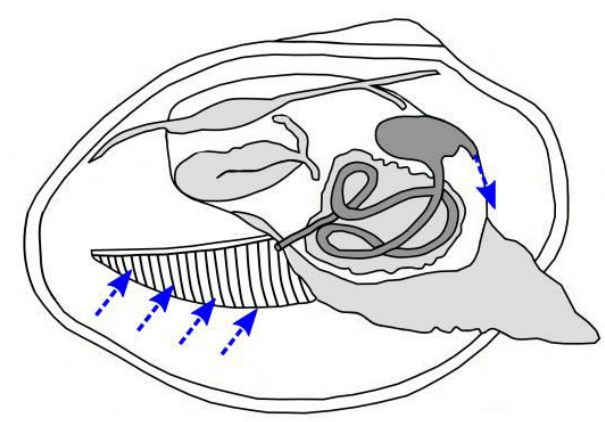

D

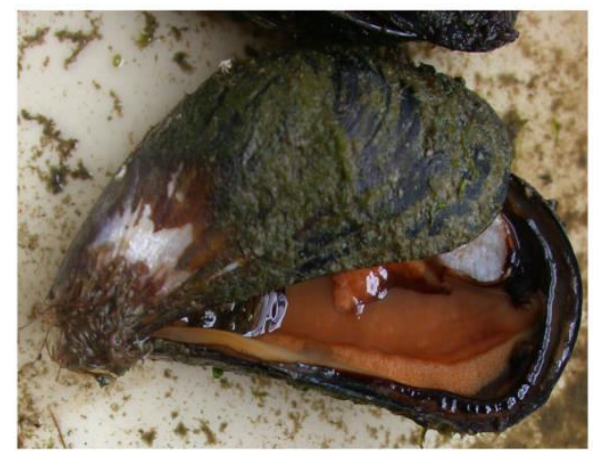

$E$

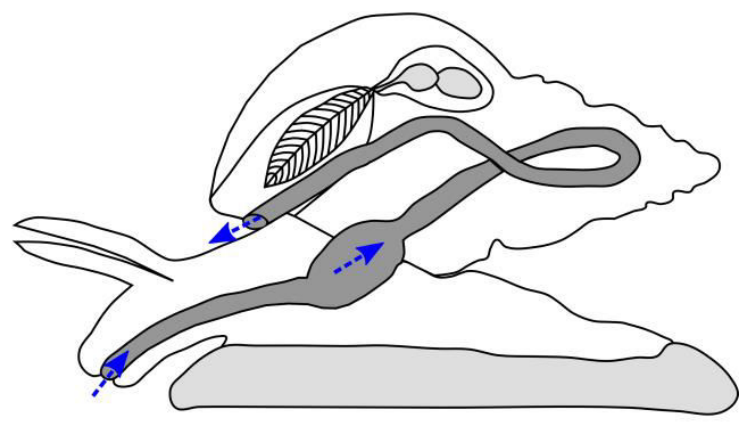

F

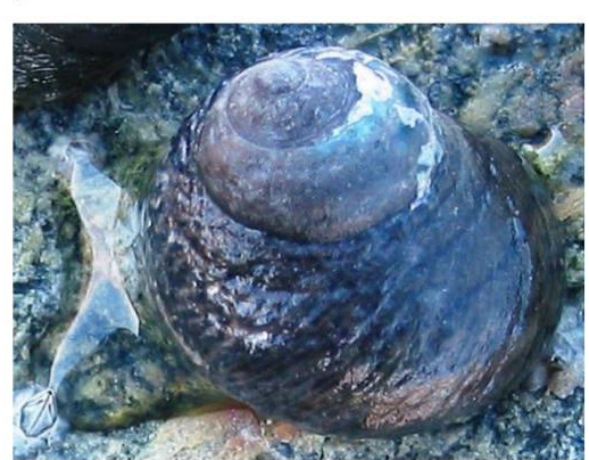

Figure 1.1 Examples of filter-feeding and surface-grazing marine invertebrates.

Schematic diagrams of the digestive tracts (dark grey) of filter-feeding tunicates (A, B), filter-feeding bivalve molluscs (C, D), and grazing gastropod molluscs (E, F) are shown. The direction of food movement is indicated by blue arrows. Examples of a filter-feeding tunicate (B), Ciona intestinalis, Phylum Chordata; a filter-feeding bivalve mollusc (D), Mytilus edulis, Phylum Mollusca; and a grazing gastropod mollusc (F), Amphibola crenata, Phylum Mollusca. Photos were provided by Chris Woods (B; National Institute of Water and Atmospheric Research, New Zealand) and Rod Asher (D, F; Cawthron Institute, New Zealand). 


\subsubsection{Detoxification pathways and their transcription level regulation}

\subsubsection{Metazoan detoxification pathways}

Metazoan organisms have specialised biochemical pathways to metabolise and eliminate potentially toxic chemicals from their body (i.e. xenobiotics; $\mathrm{Li}$ et al. 2007; Testa et al. 2012; Zanger \& Schwab 2013). Xenobiotic detoxification is accomplished by a number of enzymes with broad substrate specificities that are involved in one of the three phases of detoxification: oxidation/reduction (Phase I), conjugation (Phase II) and transport/elimination (Phase III; Figure 1.2; Yang et al. 2010; Testa et al. 2012).

\subsubsection{Induction of Phase I enzymes- vertebrates}

Many detoxification pathway genes can be induced by the xenobiotic(s) that the pathway ultimately metabolises (Xu et al. 2005; Yang et al. 2010; Testa et al. 2012; James \& Ambadapadi 2013). Such xenobiotic-mediated induction of detoxification gene expression is best characterised for Phase I cytochrome P450 (CYP) enzymes, particularly members of CYP sub-families 1-4 that are associated with xenobiotic metabolism (Yamazaki 2000; Pavek \& Dvorak 2008; Zanger \& Schwab 2013). For example, levels of human CYP3A4, an enzyme responsible for oxidising greater than $50 \%$ of medicinal drugs, are induced by a range of therapeutic compounds such as rifampicin, tamoxifen and hyperforin (Thummel \& Wilkinson 1998; Zanger et al. 2008). There exists inter-taxa variation in inductive responses to some xenobiotics (Martignoni et al. 2006). The steroidal drugs pregnenolone 16a-carbonitrile (PCN) and dexamethasone are highly efficacious CYP3A enzyme inducers in rodents, but not in humans (Martignoni et al. 2004; Vignati et al. 2004). In contrast, rifampicin is a strong inducer of human and dog CYP3A, but not of rodent CYP3A (Kocarek et al. 1995; Lu \& Li 2001). This inter-taxa variation in the response to xenobiotics suggests the possibility of adaptive evolution in the genetic elements that control expression of detoxification genes. 


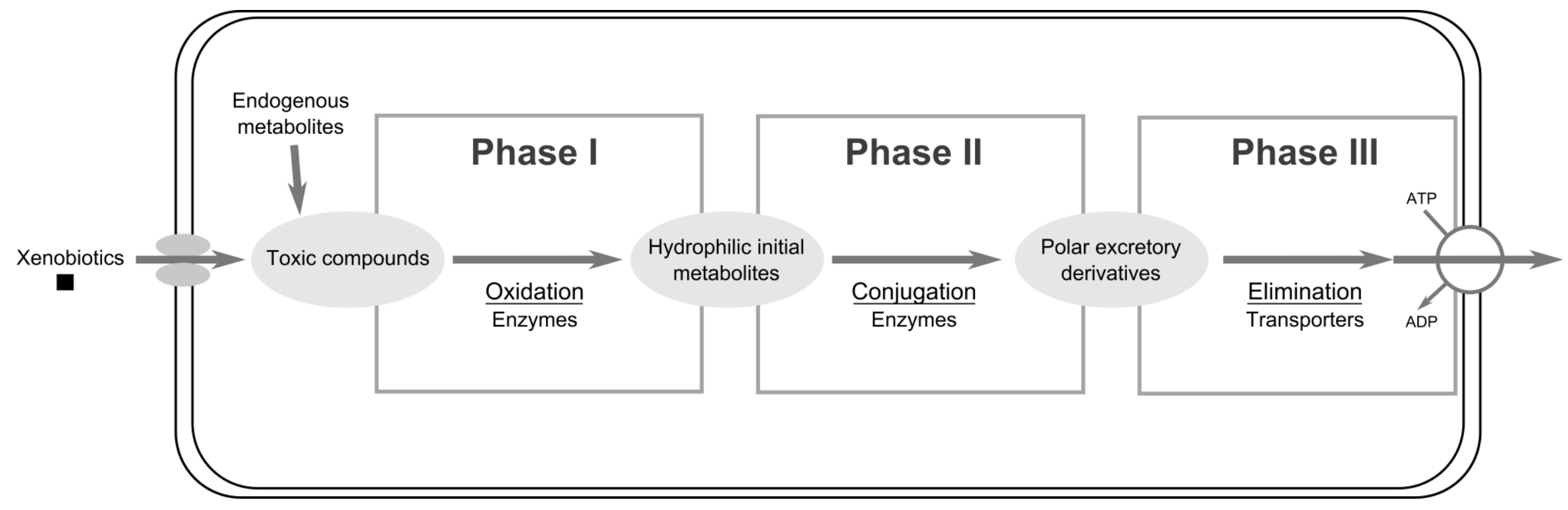

Figure 1.2 Schematic overview of metazoan detoxification pathways.

Xenobiotic detoxification is mediated via Phase I and II drug metabolising enzymes, and Phase III membrane transporters. Figure was modified from Nakata et al. (2006). 


\subsubsection{Induction of Phase I enzymes-invertebrates}

Xenobiotic-mediated induction of CYP enzyme levels has also been reported in several invertebrate phyla, especially in the Arthropoda within the context of pesticide resistance (Rewitz et al. 2006; Feyereisen 2011; Schuler \& Berenbaum 2013). In both dipteran and lepidopteran insect taxa, phenobarbital induces CYP enzymatic activity in association with transcription level induction of CYP4, CYP6 and CYP9 orthologues (Fisher et al. 2003; Natsuhara et al. 2004; Morra et al. 2010; Tomita et al. 2010). In the honey bee (Apis mellifera), aflatoxin and propolis induce CYP gene expression (Johnson et al. 2012). Amongst marine invertebrates, the polychaete Perinereis nuntia (phylum Annelida) shows increased levels of some CYP gene transcripts after exposure to benzo[a]pyrene (BaP) and polycyclic aromatic hydrocarbons (PAHs; Zheng et al. 2013). The marine gastropod Cyphoma gibbosum (phylum Mollusca) is suggested to have adapted to feeding exclusively on highly toxic gorgonian corals by differential regulation of transcripts encoding two novel CYP enzymes, CYP4BK and CYP4BL (Whalen et al. 2010a).

\subsubsection{Induction of Phase II and III enzymes}

While most xenobiotic-mediated gene induction research has focused on Phase I CYP genes, Phase II glutathione S-transferases (GSTs) and Phase III multidrug resistance-associated proteins (MRPs) have also been reported to be inducible by some xenobiotics (Oakley 2011; Bousova \& Skalova 2012). For example, expression of mouse GSTA1, MRP2 and MRP3 genes is induced by both PCN and 1,4-Bis[2-(3,5-dichloropyridyloxy)]benzene (TCPOBOP; Maglich et al. 2002). In the marine environment, dietary toxins (e.g. cyclopentenone prostaglandins) have been shown to be both inducers and substrates of GST enzymes in three marine mollusc taxa (Kuhajek \& Schlenk 2003; Whalen et al. 2008; Whalen et al. 2010b). 


\subsubsection{Xenobiotic receptors}

\subsubsection{Overview of xenobiotic receptors belonging to the nuclear receptor super-family}

It is likely that xenobiotic-mediated control of detoxification pathway gene expression may adaptively evolve in response to the various chemicals that different animals are exposed to. Diet is probably the main route of exposure to xenobiotics (Targett \& Arnold 2001; Raubenheimer \& Simpson 2009; Forbey et al. 2013). Metazoan genomes encode a range of sensors to detect xenobiotic chemicals: for example, $G$ protein-coupled receptors that mediate taste and olfaction (Palmer 2007; Kinnamon 2012). Xenobiotic detection pathways operating independently of the nervous system are coordinated through xenobiotic receptors, a specific group of ligand-activated nuclear receptor (NR) transcription factors (Nakata et al. 2006; Chai et al. 2013; Wallace \& Redinbo 2013).

Metazoan genomes encode at least three xenobiotic receptors that regulate detoxification: aryl hydrocarbon receptor (AhR), and the two NRs constitutive androstane receptor (CAR; NR notation: NR1/3) and pregnane $X$ receptor (PXR; NR notation: NR112; Nakata et al. 2006; Chai et al. 2013; Wallace \& Redinbo 2013). As is typical of NRs, the PXR and CAR protein structures consist of several conserved functional domains (Figure 1.3). The most conserved one is the DNA-binding domain (DBD), which is responsible for the interaction between the NRs and the response elements present in the promoter region of their target genes; and the ligand-binding domain (LBD), which interacts with the ligands and with co-regulators to control gene expression (Gronemeyer et al. 2004; Pascussi et al. 2008). Based on these highly conserved domains, seven NR sub-families (NR0-6) were defined using phylogenetic analyses (Nuclear Receptors Nomenclature Committee 1999). 


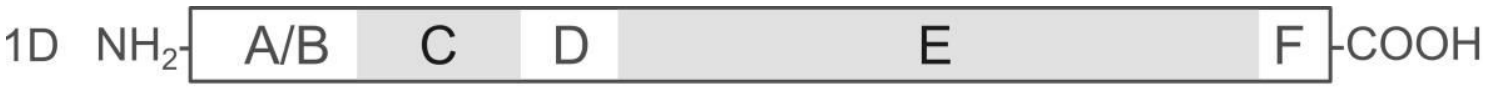

DNA-binding domain Ligand-binding domain

$3 \mathrm{D}$

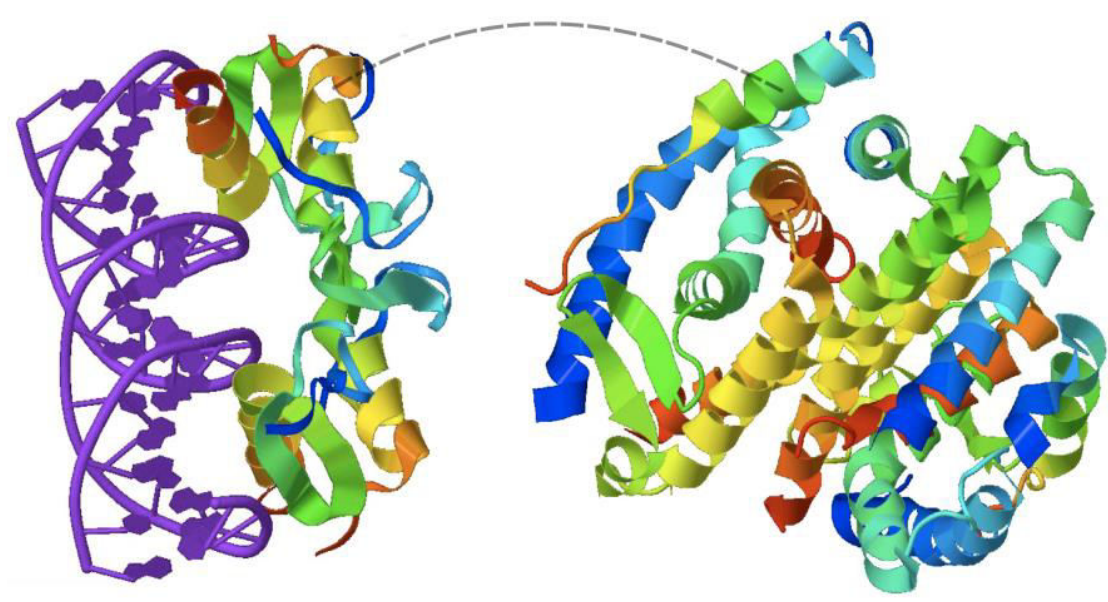

Figure 1.3 Schematic structure of nuclear receptors.

The overall structure of ligand-activated nuclear receptors is conserved through evolution with five key structural domains: N-terminal transcription activation domain (activation function 1, AF-1), DNA-binding domain (DBD), flexible hinge region (Hinge) and ligand-binding domain (LBD) which includes a C-terminal activation domain (activation function 2, AF-2). Figure was modified from Chai et al. (2013).

Technically, AhR is not a member of the NR super-family and instead belongs to the family of basic helix-loop-helix transcription factors (Kewley et al. 2004; Fuji-Kuriyama \& Kawajiri 2010). This receptor consists of four major domains, some of which are similar to that of the canonical domain composition of NRs (Figure 1.3), including a DBD and LBD (Fukunaga et al. 1995). Activated by a wide range of xenobiotic compounds, $\mathrm{AhR}$ is believed to act as a detection mechanism, sensing potentially toxic foreign compounds and facilitating their eventual metabolism and elimination (Denison \& Nagy 2003).

Pregnane $X$ receptor and CAR are the master xenobiotic sensors that bind a variety of ligands including endogenous and exogenous (i.e. xenobiotic) chemicals (Chai et al. 2013; Xie \& Chiang 2013). Although both CAR and PXR modulate a number of drug-metabolising enzymes (Kliewer et al. 2002; Kachaylo et al. 2011; Wallace \& Redinbo 2013), PXR is better understood with 
respect to how its LBD structure relates to ligand-binding and subsequent transcriptional activation (Chai et al. 2013). Crystallographic studies revealed that CAR, although promiscuous, is modulated by a smaller variety of ligands compared to the plethora that activate PXR (Wallace \& Redinbo 2013).

\subsubsection{Vertebrate pregnane $X$ receptor}

Pregnane $X$ receptor is well characterised within a number of vertebrate taxa (Reschly \& Krasowski 2006; Bainy et al. 2013; Chai et al. 2013; Xie \& Chiang 2013). Vertebrate PXR was originally identified from genomic sequence data and designated as an orphan NR as its ligand(s) were then unknown (Kliewer et al. 1998). In 1998, three groups independently reported mammalian PXR activation by both steroids and a range of xenobiotics resulting in three alternative receptor names with PXR now being the most widely used (Bertilsson et al. 1998; Blumberg et al. 1998; Kliewer et al. 1998). Pregnane $X$ receptor appears to function much like a standard ligand-activated NR. After ligand-binding within the PXR LBD, the activated PXR protein forms a complex with retinoid $X$ receptor $(R X R)$ before translocating from the cell cytoplasm into the nucleus. The PXR/RXR heterodimer binds to appropriate DNA response elements, thereby influencing transcription of adjacent genes (McKenna et al. 1999; Li \& Chiang 2005; Orans et al. 2005; Teotico et al. 2008). Many of the PXR-regulated genes are involved in detoxification. Thus, PXR activation, following xenobiotic-binding to its LBD, provides a mechanistic link between the presence of xenobiotics in a cell and appropriate detoxification gene expression (Tolson \& Wang 2010).

Vertebrate PXR ligands include a structurally diverse range of endogenously produced molecules; e.g. bile acids, steroid hormones and vitamins along with exogenously acquired chemicals; e.g. both synthetic and herbal drugs (Chang \& Waxman 2006; Staudinger et al. 2006; Manez 2008; Biswas et al. 2009; Hernandez et al. 2009; Zhou et al. 2009). Determination of the three dimensional structure of the human PXR protein has helped explain its striking permissiveness with respect to the differing structures of activating ligands 
(Wallace \& Redinbo 2013; Wu et al. 2013). In the majority of NRs, the LBD cavities have well-defined shapes with restricted mobility, thereby ensuring specificity of ligand-LBD interactions (Eick et al. 2012; Harms et al. 2013). In contrast, the human PXR LBD is larger than is typical of NRs and displays significant flexibility during ligand-binding allowing it to accommodate a wider range of ligand sizes and structures (Wallace \& Redinbo 2013; Wu et al. 2013).

\subsubsection{Tunicate xenobiotic receptors}

Although xenobiotic receptor encoding genes have been identified in a growing number of publicly-available invertebrate genomic sequences (BrackenGrissom et al. 2014), identification of marine invertebrate xenobiotic receptors is limited to a few selected taxa (Dehal et al. 2002; Reitzel \& Tarrant 2009; Bridgham et al. 2010; Denoeud et al. 2010; Srivastava et al. 2010; Vogeler et al. 2014). The first putative xenobiotic receptor from a marine invertebrate was identified in the genome of the solitary tunicate Ciona intestinalis, which was also the first marine invertebrate to have an assembled and annotated genome published (Dehal et al. 2002; Satou et al. 2002; Yagi et al. 2003). Analysis of the $C$. intestinalis genomic sequence, in combination with $C$. intestinalis expressed sequence tag (EST) databases, revealed two genes that phylogenetic analyses placed as orthologues to vertebrate NR1I genes. These two $C$. intestinalis NR1I-like genes were equally related to the vertebrate PXR, CAR and vitamin D receptor (VDR; NR notation: NR111) genes, hence denoted CNDR/PXRa (GenBank accession number: NM 001078379) and CNDR/PXR $\beta$ (NM 001044366; Dehal et al. 2002; Yagi et al. 2003; Satou et al. 2005). More recently, two putative PXR/NR1I orthologues have been detected in the genomic sequence of the colonial tunicate Botryllus schlosseri (Voskoboynik et al. 2013), while the genome of the pelagic tunicate Oikopleura dioica encodes as many as six NR1I clade genes (Denoeud et al. 2010).

The ligand-binding characteristics of the putative $C$. intestinalis PXR orthologue were subsequently investigated using a mammalian expression system (Reschly et al. 2007; Ekins et al. 2008). The CNDR/PXRa LBD was fused to the 
DBD of the generic yeast transcription factor GAL4 (GAL4-DBD) and the resulting chimeric protein was expressed in a mammalian cell line carrying a ligand-dependent luciferase reporter gene (Reschly et al. 2007; Ekins et al. 2008). Using this bioassay, three synthetic chemicals (6-formylindolo-[3,2b]carbazole, $n$-butyl-p-aminobenzoate and carbamazepine) and two microalgal biotoxins (pectenotoxin-2 (PTX-2) and okadaic acid) were identified as CNDR/PXRa LBD agonists (Reschly et al. 2007; Ekins et al. 2008; Fidler et al. 2012). A common pharmacophore model of CNDR/PXRa activators was tentatively defined. The model consists of a planar structure with at least one off-centre hydrogen bond acceptor flanked by two hydrophobic regions (Ekins et al. 2008; Fidler et al. 2012). Both microalgal biotoxins (okadaic acid and PTX-2) were two to three orders of magnitude more potent than the three synthetic ligands. This supports the idea that the natural CNDR/PXRa receptor is activated by exogenous compounds relevant to its marine environment, thus playing a similar role in detecting and eliminating xenobiotics as its vertebrate orthologue (Sladek 2011; Fidler et al. 2012). This assumption is supported by the following observations: (i) putative endogenous ligands (e.g. vitamin $D$ or bile salt correlates) have not yet been identified in invertebrates (Reschly et al. 2007; Ekins et al. 2008) and; (ii) multiple canonical xenobiotic response elements are present in the upstream genomic sequence of most CYP1 genes (CYP1A- and CYP1B/CYP1C-like) in C. intestinalis (Goldstone et al. 2007).

\subsubsection{Suitable expression systems for functional studies of nuclear receptors}

Several in vitro methods can be used for the functional characterisation of orthologues genes from different organisms, outside of the context of a whole, intact organism (Jensen et al. 2007). Recombinant proteins can be generated in bacteria, in cultured eukaryotic cells or in a cell-free system, and assayed for activity of specified substrates. While such in vitro methods tend to be faster and cheaper than studies on whole organisms, there might be only an indirect connection between in vitro receptor function and organismal phenotypes (Jensen et al. 2007). However, using mammalian cell line-based expression 
systems, NR ligands have been successfully identified and correlated to results obtained in vivo (Luo et al. 2002; Sinz et al. 2006; Chu et al. 2009).

\subsubsection{Mammalian cell lines for functional studies of nuclear receptors}

Mammalian cell line-based reporter assays, as described in Section 1.1.3.3, have been used extensively for the functional characterisation of PXR, CAR and AhR (Raucy \& Lasker 2013). The main advantage of mammalian cell lines is that they share a similar cellular/biochemical milieu with metazoan cells, which is expected to assist correct folding and functioning of NR proteins expressed from heterologous genes. Using mammalian cell lines, receptor ligands/activators can be identified using ligand-binding assays or cell-based transfection assays (Raucy \& Lasker 2013).

One popular approach for reporter assays is to make chimeric receptors containing the LBD of the receptor of interest fused to the GAL4-DBD. The reporter plasmids are then designed to respond to GAL4 (Stanley et al. 2006). This approach identified different compounds as potent activators of both the human and mouse PXR; using a GAL4-luciferase reporter construct responding to human PXR-LBD/GAL4-DBD or mouse PXR-LBD/GAL4-DBD chimeric receptors in HepG2 cells (Vignati et al. 2004). The use of a single reporter construct allows for direct comparisons of receptor-ligand interactions. However, the use of chimeric receptors means that subtle conformational changes and protein-protein interactions mediated by regions other than the LBD of the receptor of interest may be lost. For example, TCPOBOP only activated the full-length mouse CAR, but not the mouse CAR-LBD/GAL4-DBD chimeric protein. This suggests that the full-length receptor constructs represent the in vivo situation better than the GAL4-based assay (Moore et al. 2002).

To overcome these limitations, transactivation of the full-length NRs can be assessed in either transient or stable cell lines. In transient cell-lines, the transfected genetic material is not integrated into the nuclear genome. Thus, the foreign DNA is transiently expressed because it will be degraded or diluted via mitosis. In stable cell lines, a fraction of the transfected genetic material is 
introduced into the genome. The most common method involves transient transfection of the receptor and a response element-reporter gene construct. With this approach, the full-length human PXR, human CAR or human AhR cDNAs are cloned into an expression vector. In addition, chimeric luciferase, $\beta$ lactamase, or chloramphenicol acyl transferase reporter vectors are prepared containing the appropriate NR response elements (Stanley et al. 2006). Using transient transactivation systems, several studies have identified human PXR and AhR activators, and correlated these results to those obtained in vivo (Luo et al. 2002; Sinz et al. 2006; Chu et al. 2009). A number of disadvantages are associated with transient transfection assays including the inherent variability associated with transfection efficiency and the time required to perform these assays (Raucy \& Lasker 2013).

\subsubsection{Recombinant yeast strains for functional studies of nuclear receptors}

A valuable alternative expression system is the well-characterised unicellular eukaryotic organism Saccharomyces cerevisiae (common baker's yeast). Yeast is easy to cultivate and there are numerous tools available for its genetic manipulation. Due to some common characteristics with mammalian cells, yeast has become a popular organism in studying NR functions and ligand affinities (Chen et al. 2004; Fox et al. 2008; Balsiger et al. 2010; Miller et al. 2010; Raucy \& Lasker 2010; Rajasarkka \& Virta 2011; Li et al. 2013). Some of the resulting yeast strains have found application in screening environmental samples for bioactivities - particularly for estrogenic activity (Routledge \& Sumpter 1996; Collins et al. 1997; Gaido et al. 1997; Chatterjee et al. 2008; Passos et al. 2009; Balsiger et al. 2010; Chen et al. 2014). Finally, S. cerevisiae offers the possibility of directed evolution of NRs. In vitro mutagenesis can be used to generate NR LBD variant sequences, which can be selected for enhancement of growth rates in the presence of a cognate ligand (Chen \& Zhao 2003).

Despite the clear advantages of recombinant NR-based yeast bioassays, these cell-based bioassays face a number of limitations. Such limitations include the 
inability to express structurally complex proteins. Members of the growth factor beta (TGF- $\beta$ ) super-family, important proteins involved in cell growth, cell differentiation, apoptosis and cellular homeostasis, were only expressed successfully as recombinant proteins in mammalian expression systems (Kim et al. 2002). Limitations intrinsic to all living cells include elimination of potential NR ligands/test compounds via yeast export pumps (Liu et al. 1999; Dudley et al. 2000). It was shown that by removing such transporters from the yeast genome, the sensitivity of yeast bioassays can be improved (Balsiger et al. 2010). Low permeability of test compounds through the yeast cell wall and membrane can limit assay sensitivity (Lyttle et al. 1992), although NR-based yeast bioassays were shown to be highly sensitive even with very short incubation times (4-12 hours; Bovee et al. 2004).

A number of yeast systems were developed to detect macromolecular interactions: DNA-protein (one-hybrid assay), protein-protein (two-hybrid assay) and RNA-protein (three-hybrid assay; Mager \& Winderickx 2005; Vidal \& Legrain 1999). The yeast two-hybrid system represents a powerful approach for detecting protein-protein interactions in cells and, more recently, has evolved from the original yeast two-hybrid system into a method for identifying NR ligands (reviewed in Fox et al. 2008).

The 'yeast estrogen screen' is an elegant example of a modified one-hybrid method designed to identify small-molecule compounds that bind to heterologous NR proteins expressed in yeast. The human estrogen receptor alpha $(E R \alpha)$ is integrated into the main yeast chromosome. The yeast cells also contain an expression plasmid carrying the reporter gene lac $Z$ which encodes the enzyme $\beta$-galactosidase (Figure 1.4A; Routledge \& Sumpter 1996; Bovee et al. 2004). The ligand-bound ER binds the estrogen receptor response element (ERE) inserted on the promoter of the lacZ reporter gene. Then transcriptional activation of the lac $Z$ reporter takes place via recruitment of various yeast transcription cofactors (e.g. chromatin remodelling complexes or histone modification factors; Kennedy 2002).

In this approach, the native, full-length NR is being used to identify potential NR ligands. Thus, both the NR coactivators and the sequences of the cognate DNA elements to which the NR DBD binds need to be known. Such specialised 
knowledge may not be available for some NRs, especially in the case of orthologues for invertebrate xenobiotic receptors. Even though putative response elements for metazoan xenobiotic receptors have been identified in marine invertebrate genomes (Goldstone et al. 2007), these may not function as required in $S$. cerevisiae cells.

These knowledge gaps can be bypassed by exploiting the highly modular structure of NRs (Figure 1.3). A chimeric protein can be generated in which the LBD of interest is fused to the GAL4-DBD. These transcription factors contain: (i) modular DBDs that bind to specific DNA response elements and; (ii) modular transcriptional activation domains (ADs) that interact with the basal transcription machinery to increase transcriptional activation (Figure 1.4B; Raucy \& Lasker 2010). The interaction between the NR LBD and a ligand is detected using a reporter gene containing well-characterised DNA control elements (e.g. GAL4 upstream activation sequence, UAS; Young 1998). This removes the need for knowledge of the natural heterodimer partners of the NR or the DNA sequence elements to which the NR binds through its native DBD. Following binding to a well-characterised UAS element, via the GAL4-DBD, the NR's ligand-dependent activation domain (AF-2, Figure 1.3) needs to function within the nuclear milieu of yeast cells. As previous studies have shown that the AF-2 domains of some vertebrate NRs do not function in yeast cells (Berry et al. 1990; Louvion et al. 1993), a generic transcription activation domain from the Herpes simplex virion protein 16 (VP16-AD) can be added to the C-terminus of the chimeric proteins (Louvion et al. 1993). In summary, a fusion gene can be generated encoding a chimeric protein that contains the GAL4-DBD, the NR LBD and the VP16-AD, with the ligand-binding characteristics of the chimeric protein determined by the NR LBD (Figure 1.4B). 

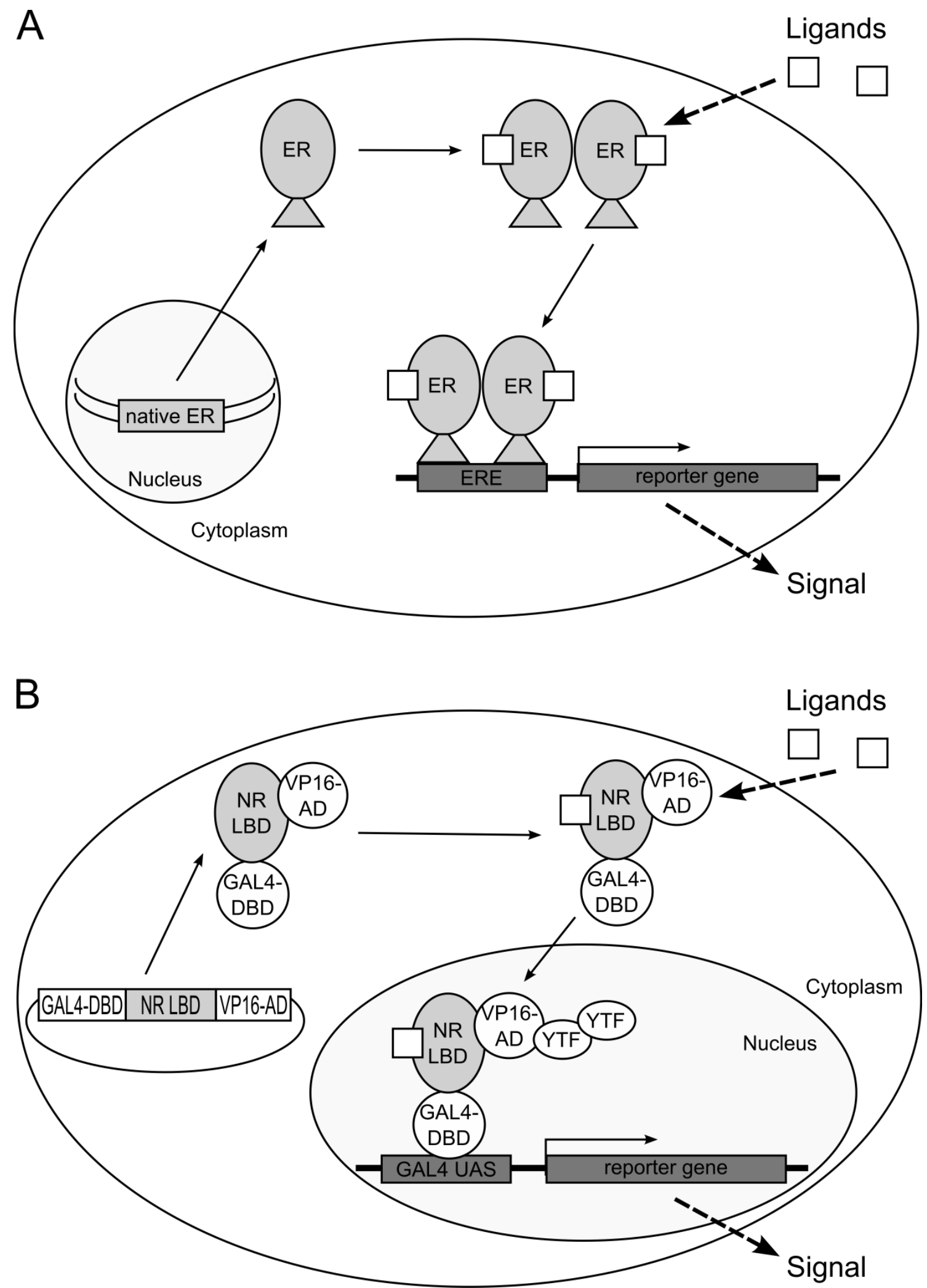

Figure 1.4 Simplified schematic of modified yeast one-hybrid bioassays.

(A) In the yeast estrogen assay the native human estrogen receptor alpha (ER $\alpha)$ is integrated into the main genome and is expressed in a form capable of binding estrogen response elements (ERE) within a hybrid promoter on the expression plasmid 
encoding the reporter gene. (B) In a modified yeast one-hybrid assay, a yeast host strain has a plasmid carrying a chimeric gene encoding a fusion protein containing the DNA-binding domain (DBD) of the yeast transcription factor GAL4 (GAL4-DBD), a nuclear receptor (NR) ligand-binding domain (LBD) and a generic activation domain (AD) from the viral protein VP16 (VP16-AD). Ligand-binding leads to a conformational change of the chimeric protein, resulting in binding of the GAL4-DBD to the control region in the promoter region of the reporter gene which is integrated in the yeast strain genome. The VP16-AD is responsible for recruitment of general yeast transcription factors (YTF).

The reporter gene selected to generate the 'output' signal from yeast bioassays must combine low background with a clear response signal following activation of the NR LBD. Three types of NR-dependent reporter gene assays have been used in recombinant yeast: the Escherichia coli lac $Z$ gene, encoding the enzyme $\beta$-galactosidase (de Almeida et al. 2008), yeast-enhanced green fluorescence protein (yEGFP; Bovee et al. 2007; Chatterjee et al. 2008; Bovee et al. 2011) and the luciferase gene (Nordeen 1988). Although the luciferase and yEGFP reporter assays are more sensitive than lacZ (Fan \& Wood 2007), both have associated complications. Luciferase assays require the use of expensive substrates and involve cell lysis (Hancock et al. 2007; de Almeida et al. 2008) which can be problematic either due to released cellular proteases (de Almeida et al. 2008) or incomplete cell lysis (Hancock et al. 2007). Although yEGFP assays do not require the addition of substrates, the assays are characterised by a high natural background of green fluorescence (Bovee et al. 2007). In contrast, lac $Z$ assays are inexpensive and, when based on the chromogenic substrate chlorophenol red- $\beta$-D-galactopyranoside (CPRG), do not require cell lysis (Routledge \& Sumpter 1996). Such non-lethal measurements of $\beta$-galactosidase activity are useful as it means that repeated measurements can be taken over time. This is a significant advantage because the time course of lacZ gene transcription induction will vary between ligands due to differences in parameters such as membrane permeability and solubility in the cytoplasm (Norcliffe et al. 2013). 


\subsection{Thesis structure and research questions}

This thesis aims to build on and extend current advances in the molecular evolution and function of a specific class of transcription regulating proteinsthe xenobiotic receptors - from marine invertebrates (Tunicata). This thesis is divided into two parts. Part one examines the molecular evolution of tunicate xenobiotic receptors in response to dietary bioactive compounds (Chapter Two). Part two focusses on the functional characterisation of tunicate xenobiotic receptors in a yeast expression system and the subsequent application of these recombinant yeast bioassays for the detection of bioactive compounds (Chapters Three, Four and Five).

The over-arching aim of this thesis was to understand the fundamental role of tunicate orthologues of the pregnane $X$ receptor $(P X R)$ and vitamin $D$ receptor (VDR) and their potential application in yeast bioassays for the detection of both bioactive compounds and environmental contaminants. This was addressed through a series of four research questions:

(i) Have tunicate VDR/PXR ligand-binding domains (LBDs) adaptively evolved to enhance binding of exogenous dietary bioactives/toxins typically encountered by these organisms?

(ii) Are tunicate VDR/PXR LBDs activated by dietary xenobiotics (e.g. microalgal biotoxins) and can these receptors be used as sensor elements in yeast bioassays for the detection of microalgal biotoxins?

(iii) Can tunicate VDR/PXR LBD-based yeast bioassays be used to detect natural bioactive compounds that represent potential drug lead compounds?

(iv) Can tunicate VDR/PXR LBD-based yeast bioassays be used to detect environmental contaminants that negatively affect a wide range of metazoan species? 
Chapter One provides a general overview of the molecular evolution and functional characterisation of metazoan PXRs with a focus on marine invertebrate PXRs.

Chapter Two documents the identification of genetic variation within two PXR orthologues in the solitary tunicate Ciona intestinalis (designated CNDR/PXR $\alpha$ and $\beta$ ) and one PXR orthologue in the colonial tunicate Botryllus schlosseri (BsVDR/PXRa).

Chapter Three describes the functional expression of two tunicate VDR/PXR orthologues genes (CNDR/PXR $\alpha$ and BsVDR/PXRa) in recombinant yeast (Saccharomyces cerevisiae) strains.

Chapter Four extends the work described in Chapter Three by screening a wide range of natural bioactive compounds and relevant analogues for activity in the tunicate VDR/PXR LBD-based yeast bioassay.

Chapter Five discusses the potential application of the tunicate VDR/PXR LBDbased yeast bioassay in environmental monitoring to detect harmful synthetic chemicals that represent emerging contaminants.

Finally, Chapter Six provides a synthesis of the proceeding chapters and a discussion on the general implications of this work and potential future research. 


\subsection{References}

NR1I2 nuclear receptor subfamily 1, group I, member 2 . Accessed on: 26 February 2014, from http://ncbi.nlm.nih.gov/gene/8856

Bainy A.C.D., Kubota A., Goldstone J.V., Lille-Langoy R., Karchner S.I., Celander M.C., Hahn M.E., Goksoyr A., Stegeman J.J. 2013. Functional characterization of a full length pregnane $X$ receptor, expression in vivo and identification of PXR alleles, in Zebrafish (Danio rerio). Aquatic Toxicology 142-143: 447-57. doi: 10.1016/j.aquatox.2013.09.014

Balsiger H.A., de la Torre R., Lee W.-Y., Cox M.B. 2010. A four-hour yeast bioassay for the direct measure of estrogenic activity in wastewater without sample extraction, concentration, or sterilization. Science of the Total Environment 408(6): 1422-9. doi: 10.1016/j.scitotenv.2009.12.027

Berry M., Metzger D., Chambon P. 1990. Role of the two activating domains of the estrogen receptor in the cell-type and promoter-context dependent agonistic activity of the antiestrogen 4-hydroxytamoxifen. EMBO Journal 9(9): 2811-8.

Bertilsson G., Heidrich J., Svensson K., Asman M., Jendeberg L., SydowBackman M., Ohlsson R., Postlind H., Blomquist P., Berkenstam A. 1998. Identification of a human nuclear receptor defines a new signaling pathway for CYP3A induction. PNAS 95(21): 12208-13. doi:

10.1073/pnas.95.21.12208

Bertrand W., Brunet F.G., Escriva H., Parmentier G., Laudet V., RobinsonRechavi M. 2004. Evolutionary genomics of nuclear receptors: from twenty-five ancestral genes to derived endocrine systems. Molecular Biology and Evolution 21(10): 1923-37. doi: 10.1093/molbev/msh200

Biswas A., Mani S., Redinbo M.R., Krasowski M.D., Li H., Ekins S. 2009. Elucidating the 'Jekyll and Hyde' nature of PXR: the case for discovering antagonists or allosteric antagonists. Pharmaceutical Research 26(8): 1807-15. doi: 10.1007/s11095-009-9901-7

Blumberg B., Sabbagh W., Juguilon H., Bolado J., van Meter C.M., Ono E.S., Evans R.M. 1998. SXR, a novel steroid and xenobiotic-sensing nuclear receptor. Genes and Development 12(20): 3195-205. doi: 10.1101/gad.12.20.3195

Bousova I., Skalova L. 2012. Inhibition and induction of glutathione Stransferases by flavonoids: possible pharmacological and toxicological consequences. Drug Metabolism Reviews 44(4): 267-86. doi: 10.3109/03602532.2012.713969

Bovee T.F., Helsdingen R.J., Rietjens I.M., Keijer J., Hoogenboom R.L. 2004. Rapid yeast estrogen bioassays stably expressing human estrogen receptors alpha and beta and green fluorescent protein: a comparison of different compounds with both receptor types. Journal of Steroid Biochemistry and Molecular Biology 91(3): 99-109. doi: 10.1016/j.jsbmb.2004.03.118

Bovee T.F., Helsdingen R.J.R., Hamers A.R.M., van Duursen M.B.M., Nielen M.W.F., Hoogenboom R.L.A.P. 2007. A new highly specific and robust yeast androgen bioassay for the detection of agonists and antagonists. Analytical and Bioanalytical Chemistry 389(5): 1549-58. doi: 10.1007/s00216-007-1559-6 
Bovee T.F., Hendriksen P.J.M., Portier L., Wang S., Elliott C.T., van Egmond H.P., Nielen M.W.F., Peijnenburg A., Hoogenboom L.A.P. 2011. Tailored microarray platform for the detection of marine toxins. Environmental Science and Technology 45(20): 8965-73. doi: 10.1021/es2011385

Bracken-Grissom H., Collins A.G., Collins T., Crandall K., Distel D., Dunn C., Giribet G., Haddock S., Knowlton N., Martindale M.et al. 2014. The Global Invertebrate Genomics Alliance (GIGA): developing community resources to study diverse invertebrate genomes. Journal of Heredity 105(1): 1-18. doi: 10.1093/jhered/est084

Bridgham J.T., Eick G.N., Larroux C., Deshpande K., Harms M.J., Gauthier M.E.A., Ortlund E.A., Degnan B.M., Thornton J.W. 2010. Protein evolution by molecular tinkering: diversification of the nuclear receptor superfamily from a ligand-dependent ancestor. PLoS Biology 8(10). doi: 10.1371/journal.pbio.1000497

Chai X., Zeng S., Xie W. 2013. Nuclear receptors PXR and CAR: implications for drug metabolism regulation, pharmacogenomics and beyond. Expert Opinion on Drug Metabolism and Toxicology 9(3): 253-66. doi: 10.1517/17425255.2013.754010

Chang T.K.H., Waxman D.J. 2006. Synthetic drugs and natural products as modulators of constitutive androstane receptor (CAR) and pregnane $X$ receptor (PXR). Drug Metabolism Reviews 38(1-2): 51-73. doi: 10.1080/03602530600569828

Chatterjee S., Kumar V., Majumder C.B., Roy P. 2008. Screening of some antiprogestin endocrine disruptors using a recombinant yeast-based in vitro bioassay. Toxicology in Vitro 22(3): 788-98. doi: 10.1016/j.tiv.2007.12.006

Chen C.H., Chou P.H., Kawanishi M., Yagi T. 2014. Occurrence of xenobiotic ligands for retinoid $X$ receptors and thyroid hormone receptors in the aquatic environment of Taiwan. Marine Pollution Bulletin 23(14). doi: 10.1016/j.marpolbul.2014.01.025

Chen Q., Chen J., Sun T., Shen J.H., Shen X., Jiang H.L. 2004. A yeast twohybrid technology-based system for the discovery of PPAR gamma agonist and antagonist. Analytical Biochemistry 335(2): 253-9. doi: 10.1016/j.ab.2004.09.004

Chen Z.L., Zhao H.M. 2003. A highly efficient and sensitive screening method for trans-activation activity of estrogen receptors. Gene 306: 127-34. doi: 10.1016/s0378-1119(03)00431-1

Chu V., Einolf H.J., Evers R., Kumar G., Moore D., Ripp S., Silva J., Sinha V., Sinz M., Skerjanec A. 2009. In vitro and in vivo induction of cytochrome P450: a survey of the current practices and recommendations: a pharmaceutical research and manufacturers of america perspective. Drug Metabolism and Disposition 37(7): 1339-54. doi: 10.1124/dmd.109.027029

Collins B.M., McLachlan J.A., Arnold S.F. 1997. The estrogenic and antiestrogenic activities of phytochemicals with human estrogen receptor expressed in yeast. Steroids 62(4): 365-72. doi: 10.1016/s0039128x(96)00246-2

de Almeida R.A., Burgess D., Shema R., Motlekar N., Napper A.D., Diamond S.L., Pavitt G.D. 2008. A Saccharomyces cerevisiae cell-based quantitative beta-galactosidase handling assay compatible with robotic high-throughput screening. Yeast 25(1): 71-6. doi: 10.1002/yea.1570 
Dearing M.D., Foley W.J., McLean S. 2005. The influence of plant secondary metabolites on the nutritional ecology of herbivorous terrestrial vertebrates. Annual Review of Ecology Evolution and Systematics 36: 169-89. doi: 10.1146/annurev.ecolsys.36.102003.152617

Dehal P., Satou Y., Campbell R.K., Chapman J., Degnan B., De Tomaso A., Davidson B., Di Gregorio A., Gelpke M., Goodstein D.M.et al. 2002. The draft genome of Ciona intestinalis: insights into chordate and vertebrate origins. Science 298(5601): 2157-67. doi: 10.1126/science.1080049

Delsuc F., Brinkmann H., Chourrout D., Philippe H. 2006. Tunicates and not cephalochordates are the closest living relatives of vertebrates. Nature 439(7079): 965-8. doi: 10.1038/nature04336

Delsuc F., Tsagkogeorga G., Lartillot N., Philippe H. 2008. Additional molecular support for the new chordate phylogeny. Genesis 46(11): 592-604. doi: $10.1002 / d v g .20450$

Denison M.S., Nagy S.R. 2003. Activation of the aryl hydrocarbon receptor by structurally diverse exogenous and endogenous chemicals. Annual Review of Pharmacology and Toxicology 43: 309-34. doi: 10.1146/annurev.pharmtox.43.100901.135828

Denoeud F., Henriet S., Mungpakdee S., Aury J.-M., Da Silva C., Brinkmann H., Mikhaleva J., Olsen L.C., Jubin C., Canestro C.et al. 2010. Plasticity of animal genome architecture unmasked by rapid evolution of a pelagic tunicate. Science 330(6009): 1381-5. doi: 10.1126/science.1194167

Dudley M.W., Sheeler C.Q., Wang H., Khan S. 2000. Activation of the human estrogen receptor by the antiestrogens $\mathrm{ICl} 182,780$ and tamoxifen in yeast genetic systems: implications for their mechanism of action. PNAS 97(7): 3696-701. doi: 10.1073/pnas.97.7.3696

Echevarria M., Naar J.P., Tomas C., Pawlik J.R. 2012. Effects of Karenia brevis on clearance rates and bioaccumulation of brevetoxins in benthic suspension feeding invertebrates. Aquatic Toxicology 106: 85-94. doi: 10.1016/j.aquatox.2011.10.011

Eick G.N., Colucci J.K., Harms M.J., Ortlund E.A., Thornton J.W. 2012. Evolution of minimal specificity and promiscuity in steroid hormone receptors. PLoS Genetics 8(11). doi: 10.1371/journal.pgen.1003072

Ekins S., Reschly E.J., Hagey L.R., Krasowski M.D. 2008. Evolution of pharmacologic specificity in the pregnane $\mathrm{X}$ receptor. BMC Evolutionary Biology 8. doi: 10.1186/1471-2148-8-103

Fan F., Wood K.V. 2007. Bioluminescent assays for high-throughput screening. Assay and Drug Development Technologies 5(1): 127-36. doi: 10.1089/adt.2006.053

Fernandez-Reiriz M.J., Navarro J.M., Contreras A.M., Labarta U. 2008. Trophic interactions between the toxic dinoflagellate Alexandrium catenella and Mytilus chilensis: feeding and digestive behaviour to long-term exposure. Aquatic Toxicology 87(4): 245-51. doi: 10.1016/j.aquatox.2008.02.011

Feyereisen R. 2011. Arthropod CYPomes illustrate the tempo and mode in P450 evolution. Biochimica et Biophysica Acta 1814(1): 19-28. doi: 10.1016/j.bbapap.2010.06.012

Fidler A.E., Holland P.T., Reschly E.J., Ekins S., Krasowski M.D. 2012. Activation of a tunicate (Ciona intestinalis) xenobiotic receptor orthologue by both natural toxins and synthetic toxicants. Toxicon 59: 365-72. doi: 10.1016/j.toxicon.2011.12.008 
Fisher T., Crane M., Callaghan A. 2003. Induction of cytochrome P450 activity in individual Chironomus riparius (Meigen) larvae exposed to xenobiotics. Ecotoxicology and Environmental Safety 54(1): 1-6. doi: 10.1016/s01476513(02)00031-3

Forbey J.S., Dearing M.D., Gross E.M., Orians C.M., Sotka E.E., Foley W.J. 2013. A Pharm-Ecological perspective of terrestrial and aquatic plantherbivore interactions. Journal of Chemical Ecology 39(4): 465-80. doi: 10.1007/s10886-013-0267-2

Fox J.E., Burow M.E., McLachlan J.A., Miller C.A. 2008. Detecting ligands and dissecting nuclear receptor-signaling pathways using recombinant strains of the yeast Saccharomyces cerevisiae. Nature Protocols 3(4): 637-45. doi: 10.1038/nprot.2008.33

Fuji-Kuriyama Y., Kawajiri K. 2010. Molecular mechanisms of the physiological functions of the aryl hydrocarbon (dioxin) receptor, a multifunctional regulator that senses and responds to environmental stimuli. PNAS 86(1): 40-53. doi: 10.2183/pjab.86.40

Fukunaga B.N., Probst M.R., Reisz-Porszasz S., Hankinson O. 1995. Identification of functional domains of the aryl hydrocarbon receptor. Journal of Biological Chemistry 270(49): 29270-8. doi: $10.1074 / \mathrm{jbc} .270 .49 .29270$

Gaido K.W., Leonard L.S., Lovell S., Gould J.C., Babai D., Portier C.J., McDonnell D.P. 1997. Evaluation of chemicals with endocrine modulating activity in a yeast-based steroid hormone receptor gene transcription assay. Toxicology and Applied Pharmacology 143(1): 205-12. doi: 10.1006/taap.1996.8069

Glendinning J.I. 2007. How do predators cope with chemically defended foods? Biological Bulletin 213(3): 252-66.

Goldstone J.V., Goldstone H.M.H., Morrison A.M., Tarrant A., Kern S.E., Woodin B.R., Stegeman J.J. 2007. Cytochrome P450 1 genes in early deuterostomes (tunicates and sea urchins) and vertebrates (chicken and frog): origin and diversification of the CYP1 gene family. Molecular Biology and Evolution 24(12): 2619-31. doi: 10.1093/molbev/msm200

Gronemeyer H., Gustafsson J.A., Laudet V. 2004. Principles for modulation of the nuclear receptor superfamily. Nature Reviews Drug Discovery 3(11): 950-64. doi: 10.1038/nrd1551

Haberkorn H., Tran D., Massabuau J.C., Ciret P., Savar V., Soudant P. 2011. Relationship between valve activity, microalgae concentration in the water and toxin accumulation in the digestive gland of the Pacific oyster Crassostrea gigas exposed to Alexandrium minutum. Marine Pollution Bulletin 62(6): 1191-7. doi: 10.1016/j.marpolbul.2011.03.034

Hancock M.K., Medina M.N., Smith B.M., Orth A.P. 2007. Microplate orbital mixing improves high-throughput cell-based reporter assay readouts. Journal of Biomolecular Screening 12(1): 140-4. doi: $10.1177 / 1087057106296046$

Harms M.J., Eick G.N., Goswami D., Colucci J.K., Griffin P.R., Ortlund E.A., Thornton J.W. 2013. Biophysical mechanisms for large-effect mutations in the evolution of steroid hormone receptors. PNAS 110(28): 11475-80. doi: $10.1073 /$ pnas. 1303930110

Hay M.E. 2009. Marine chemical ecology: chemical signals and cues structure marine populations, communities and ecosystems. Annual Review of 
Marine Science 1: 193-212. doi:

10.1146/annurev.marine.010908.163708

Hegaret H., Wikfors G.H., Shumway S.E. 2007. Diverse feeding responses of five species of bivalve mollusc when exposed to three species of harmful algae. Journal of Shellfish Research 26(2): 549-59. doi: 10.2983/07308000(2007)26

Hernandez J.P., Mota L.C., Baldwin W.S. 2009. Activation of CAR and PXR by dietary, environmental and occupational chemicals alters drug metabolism, intermediary metabolism and cell proliferation. Current Pharmacogenomics and Personalized Medicine 7(2): 81-105. doi: $10.2174 / 187569209788654005$

James M.O., Ambadapadi S. 2013. Interactions of cytosolic sulfotransferases with xenobiotics. Drug Metabolism Reviews 45(4): 401-14. doi: 10.3109/03602532.2013.835613

Jensen J.D., Wong A., Aquadro C.F. 2007. Approaches for identifying targets of positive selection. Trends in Genetics 23(11): 568-77. doi: 10.1016/j.tig.2007.08.009

Johnson R.M., Mao W., Pollock H.S., Niu G., Schuler M.A., Berenbaum M.R. 2012. Ecologically appropriate xenobiotics induce cytochrome P450s in Apis mellifera. PLoS One 7(2). doi: 10.1371/journal.pone.0031051

Kachaylo E.M., Pustylnyak V.O., Lyakhovich V.V., Gulyaeva L.F. 2011. Constitutive androstane receptor (CAR) is a xenosensor and target for therapy. Biochemistry 76(10): 1087-97. doi:

$10.1134 /$ S0006297911100026

Kennedy B.K. 2002. Mammalian transcription factors in yeast: strangers in a familiar land. Nature Reviews Molecular Cell Biology 3(1): 41-9. doi: 10.1038/nrm704

Kewley R.J., Whitelaw M.L., Chapman-Smith A. 2004. The mammalian basic helix-loop-helix/PAS family of transcriptional regulators. International Journal of Biochemistry and Cell Biology 36(2): 189-204. doi: 10.1016/s1357-2725(03)00211-5

Kim M.S., Ahn S.M., Moon A. 2002. In vitro bioassay for transforming growth factor-beta using XTT method. Archives of Pharmacal Research 25(6): 903-9. doi: 10.1007/BF02977012

Kinnamon S.C. 2012. Taste receptor signalling - from tongues to lungs. Acta Physiologica 204(2): 158-68. doi: 10.1111/j.1748-1716.2011.02308

Kliewer S., Goodwin B., Willson T. 2002. The nuclear pregnane X receptor: a key regulator of xenobiotic metabolism. Endocrine Reviews 23: 687-702. doi: 10.1210/er.2001-0038

Kliewer S.A., Moore J.T., Wade L., Staudinger J.L., Watson M.A., Jones S.A., McKee D.D., Oliver B.B., Willson T.M., Zetterstrom R.H.et al. 1998. An orphan nuclear receptor activated by pregnanes defines a novel steroid signaling pathway. Cell 92(1): 73-82. doi: 10.1016/s00928674(00)80900-9

Kocarek T.A., Schuetz E.G., Strom S.C., Fisher R.A., Guzelian P.S. 1995. Comparative analysis of cytochrome P4503A induction in primary cultures of rat, rabbit and human hepatocytes. Drug Metabolism and Disposition 23(3): 415-21.

Krasowski M.D., Yasuda K., Hagey L.R., Schuetz E.G. 2005a. Evolutionary selection across the nuclear hormone receptor superfamily with a focus on the NR1I subfamily (vitamin D, pregnane $X$ and constitutive 
androstane receptors). Nuclear Receptor 3: 2. doi: 10.1186/1478-1336-32

Krasowski M.D., Yasuda K., Hagey L.R., Schuetz E.G. 2005b. Evolution of the pregnane $\mathrm{X}$ receptor: adaptation to cross-species differences in biliary bile salts. Molecular Endocrinology 19(7): 1720-39. doi: 10.1210/me.2004-0427

Krasowski M.D., Ni A., Hagey L.R., Ekins S. 2011a. Evolution of promiscuous nuclear hormone receptors: LXR, FXR, VDR, PXR and CAR. Molecular and Cellular Endocrinology 334(1-2): 39-48. doi: 10.1016/j.mce.2010.06.016

Krasowski M.D., Ai N., Hagey L.R., Kollitz E.M., Kullman S.W., Reschly E.J., Ekins S. 2011b. The evolution of farnesoid $X$, vitamin $D$ and pregnane $X$ receptors: insights from the green-spotted pufferfish (Tetraodon nigriviridis) and other non-mammalian species. BMC Biochemistry 12. doi: 10.1186/1471-2091-12-5

Kuhajek J.M., Schlenk D. 2003. Effects of the brominated phenol, lanosol, on cytochrome P450 and glutathione transferase activities in Haliotis rufescens and Katharina tunicata. Comparative Biochemistry and Physiology 134(4): 473-9. doi: 10.1016/S1532-0456(03)00040-1

Landsberg J.H., Flewelling L.J., Naar J. 2009. Karenia brevis red tides, brevetoxins in the food web and impacts on natural resources: decadal advancements. Harmful Algae 8(4): 598-607. doi: 10.1016/j.hal.2008.11.010

Li H., Redinbo M.R., Venkatesh M., Ekins S., Chaudhry A., Bloch N., Negassa A., Mukherjee P., Kalpana G., Mani S. 2013. Novel yeast-based strategy unveils antagonist binding regions on the nuclear xenobiotic receptor PXR. Journal of Biological Chemistry 288(19): 13655-68. doi: 10.1074/jbc.M113.455485

Li T.G., Chiang J.Y.L. 2005. Mechanism of rifampicin and pregnane $X$ receptor inhibition of human cholesterol 7 alpha-hydroxylase gene transcription. American Journal of Physiology-Gastrointestinal and Liver Physiology 288(1): G74-G84. doi: 10.1152/ajpgi.00258.2004

Li X.C., Schuler M.A., Berenbaum M.R. 2007. Molecular mechanisms of metabolic resistance to synthetic and natural xenobiotics. Annual Review of Entomology 52: 231-53. doi: 10.1146/annurev.ento.51.110104.151104

Liu J.W., Jeannin E., Picard D. 1999. The anti-estrogen hydroxytamoxifen is a potent antagonist in a novel yeast system. Journal of Biological Chemistry 380(11): 1341-5. doi: 10.1515/BC.1999.172

Long J.D., Hay M.E. 2006. Fishes learn aversions to a nudibranch's chemical defense. Marine Ecology Progress Series 307: 199-208. doi: 10.3354/meps307199

Louvion J.F., Havauxcopf B., Picard D. 1993. Fusion of GAL4-VP16 to a steroid-binding domain provides a tool for gratuitous induction of galactose-responsive genes in yeast. Gene 131(1): 129-34. doi:10.1016/0378-1119(93)90681-R

Lu C., Li A.P. 2001. Species comparison in P450 induction: effects of dexamethasone, omeprazole and rifampin on P450 isoforms $1 \mathrm{~A}$ and $3 \mathrm{~A}$ in primary cultured hepatocytes from man, Sprague-Dawley rat, minipig and beagle dog. Chemico-Biological Interactions 134(3): 271-81. doi: 10.1016/s0009-2797(01)00162-4 
Luo G., Cunningham M., Kim S., Burn T., Lin J., Sinz M., Hamilton G., Rizzo C., Jolley S., Gilbert D.et al. 2002. CYP3A4 induction by drugs: correlation between a pregnane $\mathrm{X}$ receptor reporter gene assay and CYP3A4 expression in human hepatocytes. Drug Metabolism and Disposition 30(7): 795-804. doi: 10.1124/dmd.30.7.795

Lyttle C.R., Damianmatsumura P., Juul H., Butt T.R. 1992. Human estrogen receptor regulation in a yeast model system and studies on receptor agonists and antagonists. Journal of Steroid Biochemistry and Molecular Biology 42(7): 677-85. doi: 10.1016/0960-0760(92)90108-u

Mager W.H., Winderickx J. 2005. Yeast as a model for medical and medicinal research. Trends in Pharmacological Sciences 26(5): 265-73. doi: 10.1016/j.tips.2005.03.004

Maglich J.M., Stoltz C.M., Goodwin B., Hawkins-Brown D., Moore J.T., Kliewer S.A. 2002. Nuclear pregnane $X$ receptor and constitutive androstane receptor regulate overlapping but distinct sets of genes involved in xenobiotic detoxification. Molecular Pharmacology 62(3): 638-46. doi: 10.1124/mol.62.3.638

Manez S. 2008. A fresh insight into the interaction of natural products with pregnane $X$ receptor. Natural Product Communications 3(12): 2123-8.

Manfrin C., De Moro G., Torboli V., Venier P., Pallavicini A., Gerdol M. 2012. Physiological and molecular responses of bivalves to toxic dinoflagellates. Invertebrate Survival Journal 9(2): 184-99.

Marsh K.J., Wallis I.R., Andrew R.L., Foley W.J. 2006. The detoxification limitation hypothesis: where did it come from and where is it going? Journal of Chemical Ecology 32(6): 1247-66. doi: 10.1007/s10886-0069082-3

Martignoni M., Groothuis G.M.M., de Kanter R. 2006. Species differences between mouse, rat, dog, monkey and human CYP-mediated drug metabolism, inhibition and induction. Expert Opinion on Drug Metabolism and Toxicology 2(6): 875-94. doi: 10.1517/17425255.2.6.875

Martignoni M., de Kanter R., Grossi P., Mahnke A., Saturno G., Monshouwer M. 2004. An in vivo and in vitro comparison of CYP induction in rat liver and intestine using slices and quantitative RT-PCR. Chemico-Biological Interactions 151(1): 1-11. doi: 10.1016/j.cbi.2004.10.002

Mayer A.M.S. 2009. Special issue on marine toxins. Marine Drugs 7: 19-23. doi: $10.3390 / \mathrm{md} 7010019$

McKenna N.J., Lanz R.B., O'Malley B.W. 1999. Nuclear receptor coregulators: cellular and molecular biology. Endocrine Reviews 20(3): 321-44. doi: 10.1210/er.20.3.321

Miller A.M., Pawlik J.R. 2013. Do coral reef fish learn to avoid unpalatable prey using visual cues? Animal Behaviour 85(2): 339-47. doi: 10.1016/j.anbehav.2012.11.002

Miller C.A., Tan X.B., Wilson M., Bhattacharyya S., Ludwig S. 2010. Single plasmids expressing human steroid hormone receptors and a reporter gene for use in yeast signaling assays. Plasmid 63(2): 73-8. doi: 10.1016/j.plasmid.2009.11.003

Moore L.B., Maglich J.M., McKee D.D., Wisely B., Willson T.M., Kliewer S.A., Lambert M.H., Moore J.T. 2002. Pregnane X receptor (PXR), constitutive androstane receptor (CAR) and benzoate $X$ receptor $(B X R)$ define three pharmacologically distinct classes of nuclear receptors. Molecular Endocrinology 16(5): 977-86. doi: 10.1210/me.16.5.977 
Moore L.B., Parks D.J., Jones S.A., Bledsoe R.K., Consler T.G., Stimmel J.B., Goodwin B., Liddle C., Blanchard S.G., Willson T.M. et al. 2000. Orphan nuclear receptors constitutive androstane receptor and pregnane $X$ receptor share xenobiotic and steroid ligands. Journal of Biological Chemistry 275(20): 15122-7. doi: 10.1074/jbc.M001215200

Morra R., Kuruganti S., Lam V., Lucchesi J.C., Ganguly R. 2010. Functional analysis of the cis-acting elements responsible for the induction of the Cyp6a8 and Cyp6g1 genes of Drosophila melanogaster by DDT, phenobarbital and caffeine. Insect Molecular Biology 19(1): 121-30. doi: 10.1111/j.1365-2583.2009.00954.x

Nakata K., Tanaka Y., Nakano T., Adachi T. 2006. Nuclear receptor-mediated transcriptional regulation in phase I, II and III xenobiotic metabolizing systems. Drug Metabolism and Pharmacokinetics 21(6): 437-57. doi: 10.2133/dmpk.21.437

Natsuhara K., Shimada K., Tanaka T., Miyata T. 2004. Phenobarbital induction of permethrin detoxification and phenobarbital metabolism in susceptible and resistant strains of the beet armyworm Spodoptera exigua (Hubner). Pesticide Biochemistry and Physiology 79(2): 33-41. doi: 10.1016/j.pestbp.2004.04.002

Norcliffe J.L., Alvarez-Ruiz E., Martin-Plaza J.J., Steel P.G., Denny P.W. 2013. The utility of yeast as a tool for cell-based, target-directed highthroughput screening. Parasitology 141(1): 8-16. doi: $10.1017 / \mathrm{s} 0031182013000425$

Nordeen S.K. 1988. Luciferase reporter gene vectors for analysis of promoters and enhancers. Biotechniques 6(5): 454-8.

Nuclear Receptors Nomenclature Committee. 1999. A unified nomenclature system for the nuclear receptor superfamily. Cell 97(2): 161-3.

Oakley A. 2011. Glutathione transferases: a structural perspective. Drug Metabolism Reviews 43(2): 138-51. doi: 10.3109/03602532.2011.558093

Orans J., Teotico D.G., Redinbo M.R. 2005. The nuclear xenobiotic receptor pregnane $\mathrm{X}$ receptor: recent insights and new challenges. Molecular Endocrinology 19(12): 2891-900. doi: 10.1210/me.2005-0156

Ortiz-Ramirez F.A., Vallim M.A., Cavalcanti D.N., Teixeira V.L. 2013. Effects of the secondary metabolites from Canistrocarpus cervicornis (Dictyotales, Phaeophyceae) on fertilization and early development of the sea urchin Lytechinus variegatus. Latin American Journal of Aquatic Research 41(2): 296-304. doi: 10.3856/vol41-issue2-fulltext-8

Palmer R.K. 2007. The pharmacology and signaling of bitter, sweet and umami taste sensing. Molecular Interventions 7(2): 87-98. doi: 10.1124/mi.7.2.9

Pascussi J.-M., Gerbal-Chaloin S., Duret C., Daujat-Chavanieu M., Vilarem M.J., Maurel P. 2008. The tangle of nuclear receptors that controls xenobiotic metabolism and transport: crosstalk and consequences. Annual Review of Pharmacology and Toxicology 48: 1-32. doi: 10.1146/annurev.pharmtox.47.120505.105349

Passos A.L., Pinto P.I., Power D.M., Canario A.V. 2009. A yeast assay based on the gilthead sea bream (teleost fish) estrogen receptor beta for monitoring estrogen mimics. Ecotoxicology and Environmental Safety 72(5): 1529-37. doi: 10.1016/j.ecoenv.2009.02.004

Pavek P., Dvorak Z. 2008. Xenobiotic-induced transcriptional regulation of xenobiotic metabolizing enzymes of the cytochrome P450 superfamily in 
human extrahepatic tissues. Current Drug Metabolism 9(2): 129-43. doi: $10.2174 / 138920008783571774$

Rajasarkka J., Virta M. 2011. Miniaturization of a panel of high throughput yeast-cell-based nuclear receptor assays in 384-and 1536-well microplates. Combinatorial Chemistry and High Throughput Screening 14(1): 47-54. doi: 10.2174/1386207311107010047

Raubenheimer D., Simpson S.J. 2009. Nutritional PharmEcology: doses, nutrients, toxins and medicines. Integrative and Comparative Biology 49(3): 329-37. doi: 10.1093/icb/icp050

Raucy J.L., Lasker J.M. 2010. Current in vitro high-throughput screening approaches to assess nuclear receptor activation. Current Drug Metabolism 11(9): 806-14. doi: 10.2174/138920010794328896

Raucy J.L., Lasker J.M. 2013. Cell-based systems to assess nuclear receptor activation and their use in drug development. Drug Metabolism Reviews 45(1): 101-9. doi: 10.3109/03602532.2012.737333

Reitzel A.M., Tarrant A.M. 2009. Nuclear receptor complement of the cnidarian Nematostella vectensis: phylogenetic relationships and developmental expression patterns. BMC Evolutionary Biology 9: 230. doi: 10.1186/1471-2148-9-230

Reschly E.J., Krasowski M.D. 2006. Evolution and function of the NR1I nuclear hormone receptor subfamily (VDR, PXR and CAR) with respect to metabolism of xenobiotics and endogenous compounds. Current Drug Metabolism 7(4): 349-65. doi: 10.2174/138920006776873526

Reschly E.J., Bainy A.C.D., Mattos J.J., Hagey L.R., Bahary N., Mada S.R., Ou J., Venkataramanan R., Krasowski M.D. 2007. Functional evolution of the vitamin $\mathrm{D}$ and pregnane $\mathrm{X}$ receptors. BMC Evolutionary Biology 7 : 222. doi: 10.1186/1471-2148-7-222

Rewitz K.F., Styrishave B., Lobner-Olesen A., Andersen O. 2006. Marine invertebrate cytochrome P450: emerging insights from vertebrate and insect analogies. Comparative Biochemistry and Physiology 143(4): 36381. doi: 10.1016/j.cbpc.2006.04.001

Routledge E.J., Sumpter J.P. 1996. Estrogenic activity of surfactants and some of their degradation products assessed using a recombinant yeast screen. Environmental Toxicology and Chemistry 15(3): 241-8. doi: 10.1002/etc.5620150303

Satoh N., Rokhsar D., Nishikawa T. 2014. Chordate evolution and the threephylum system. Proceedings of the Royal Society B-Biological Sciences 281(1794): 41729-. doi: 10.1098/rspb.2014.1729

Satou Y., Kawashima T., Shoguchi E., Nakayama A., Satoh N. 2005. An integrated database of the ascidian, Ciona intestinalis: towards functional genomics. Zoological Science 22(8): 837-43. doi: 10.2108/zsj.22.837

Satou Y., Yamada L., Mochizuki Y., Takatori N., Kawashima T., Sasaki A., Hamaguchi M., Awazu S., Yagi K., Sasakura Y.et al. 2002. A cDNA resource from the basal chordate Ciona intestinalis. Genesis 33(4): 1534. doi: 10.1002/gene.10119

Schuler M.A., Berenbaum M.R. 2013. Structure and function of cytochrome $P 450$ s in insect adaptation to natural and synthetic toxins: insights gained from molecular modeling. Journal of Chemical Ecology 39(9): 1232-45. doi: 10.1007/s10886-013-0335-7

Sinz M., Kim S., Zhu Z., Chen T., Anthony M., Dickinson K., Rodrigues A.D. 2006. Evaluation of 170 xenobiotics as transactivators of human 
pregnane $\mathrm{X}$ receptor (hPXR) and correlation to known CYP3A4 drug interactions. Current Drug Metabolism 7(4): 375-88. doi: $10.2174 / 138920006776873535$

Sladek F.M. 2011. What are nuclear receptor ligands? Molecular and Cellular Endocrinology 334(1-2): 3-13. doi: 10.1016/j.mce.2010.06.018

Sotka E.E., Gantz J. 2013. Preliminary evidence that the feeding rates of generalist marine herbivores are limited by detoxification rates. Chemoecology 23(4): 233-40. doi: 10.1007/s00049-013-0137-y

Srivastava M., Simakov O., Chapman J., Fahey B., Gauthier M.E.A., Mitros T., Richards G.S., Conaco C., Dacre M., Hellsten U.et al. 2010. The Amphimedon queenslandica genome and the evolution of animal complexity. Nature 466(7307): 720-6. doi: 10.1038/nature09201

Stanley L.A., Horsburgh B.C., Ross J., Scheer N., Wolf C.R. 2006. PXR and CAR: nuclear receptors which play a pivotal role in drug disposition and chemical toxicity. Drug Metabolism Reviews 38(3): 515-97. doi: $10.1080 / 03602530600786232$

Staudinger J.L., Ding X., Lichti K. 2006. Pregnane X receptor and natural products: beyond drug-drug interactions. Expert Opinion on Drug Metabolism and Toxicology 2(6): 847-57. doi: 10.1517/17425255.2.6.847

Targett N.M., Arnold T.M. 2001. Effects of secondary metabolites on digestion in marine herbivores. In: McClintock JB, Baker BJ ed. Marine Chemical Ecology. First ed. Boca Raton, FL, CRC Press. Pp. 391-411.

Teotico D.G., Frazier M.L., Ding F., Dokholyan N.V., Temple B.R., Redinbo M.R. 2008. Active nuclear receptors exhibit highly correlated AF-2 domain motions. PLoS Computational Biology 4(7): 1000111. doi: 10.1371/journal.pcbi.1000111

Testa B., Pedretti A., Vistoli G. 2012. Foundation review: reactions and enzymes in the metabolism of drugs and other xenobiotics. Drug Discovery Today 17(11-12): 549-60. doi: 10.1016/j.drudis.2012.01.017

Thummel K.E., Wilkinson G.R. 1998. In vitro and in vivo drug interactions involving human CYP3A. Annual Review of Pharmacology and Toxicology 38: 389-430. doi: 10.1146/annurev.pharmtox.38.1.389

Tolson A.H., Wang H.B. 2010. Regulation of drug-metabolizing enzymes by xenobiotic receptors: PXR and CAR. Advanced Drug Delivery Reviews 62(13): 1238-49. doi: 10.1016/j.addr.2010.08.006

Tomita T., Itokawa K., Komagata O., Kasai S. 2010. Overexpression of cytochrome P450 genes in insecticide-resistant mosquitoes. Journal of Pesticide Science 35(4): 562-8. doi: 10.1584/jpestics.W10-45

Vidal M., Legrain P. 1999. Yeast forward and reverse ' $n$ '-hybrid systems. Nucleic Acids Research 27(4): 919-29. doi: 10.1093/nar/27.4.919

Vignati L.A., Bogni A., Grossi P., Monshouwer M. 2004. A human and mouse pregnane $X$ receptor reporter gene assay in combination with cytotoxicity measurements as a tool to evaluate species-specific CYP3A induction. Toxicology 199(1): 23-33. doi: 10.1016/j.tox.2003.12.018

Vogeler S., Galloway T., Lyons B., Bean T. 2014. The nuclear receptor gene family in the Pacific oyster, Crassostrea gigas, contains a novel subfamily group. BMC Genomics 15(1): 369. doi: 10.1186/1471-2164-15369

Voskoboynik A., Neff N.F., Sahoo D., Newman A.M., Pushkarev D., Koh W., Passarelli B., Fan H.C., Mantalas G.L., Palmeri K.J.et al. 2013. The 
genome sequence of the colonial chordate, Botryllus schlosseri. eLife 2. doi: 10.7554/eLife.00569

Wallace B.D., Redinbo M.R. 2013. Xenobiotic-sensing nuclear receptors involved in drug metabolism: a structural perspective. Drug Metabolism Reviews 45(1): 79-100. doi: 10.3109/03602532.2012.740049

Whalen K.E., Starczak V.R., Nelson D.R., Goldstone J.V., Hahn M.E. 2010a. Cytochrome P450 diversity and induction by gorgonian allelochemicals in the marine gastropod Cyphoma gibbosum. BMC Ecology 10: 24. doi: 10.1186/1472-6785-10-24

Whalen K.E., Lane A.L., Kubanek J., Hahn M.E. 2010b. Biochemical warfare on the reef: the role of glutathione transferases in consumer tolerance of dietary prostaglandins. PLoS One 5(1): 0008537. doi:

10.1371/journal.pone.0008537

Whalen K.E., Morin D., Lin C.Y., Tjeerdema R.S., Goldstone J.V., Hahn M.E. 2008. Proteomic identification, cDNA cloning and enzymatic activity of glutathione S-transferases from the generalist marine gastropod, Cyphoma gibbosum. Archives of Biochemistry and Biophysics 478(1): 717. doi: 10.1016/j.abb.2008.07.007

Wu B., Li S., Dong D. 2013. 3D structures and ligand specificities of nuclear xenobiotic receptors CAR, PXR and VDR. Drug Discovery Today 18(1112): 574-81. doi: 10.1016/j.drudis.2013.01.001

Xie W., Chiang J.Y.L. 2013. Nuclear receptors in drug metabolism and beyond. Drug Metabolism Reviews 45(1): 1-2. doi: 10.3109/03602532.2013.754577

Xu C.J., Li C.Y.T., Kong A.N.T. 2005. Induction of phase I, II and III drug metabolism/transport by xenobiotics. Archives of Pharmaceutical Research 28(3): 249-68. doi: 10.1007/bf02977789

Yagi K., Satou Y., Mazet F., Shimeld S.M., Degnan B., Rokhsar D., Levine M., Kohara Y., Satoh N. 2003. A genomewide survey of developmentally relevant genes in Ciona intestinalis - III. Genes for Fox, ETS, nuclear receptors and NF kappa B. Development Genes and Evolution 213(5-6): 235-44. doi: 10.1007/s00427-003-0322-z

Yamazaki H. 2000. Roles of human cytochrome P450 enzymes involved in drug metabolism and toxicological studies. Journal of the Pharmaceutical Society of Japan 120(12): 1347-57.

Yang Y.M., Noh K., Han C.Y., Kim S.G. 2010. Transactivation of genes encoding for phase II enzymes and phase III transporters by phytochemical antioxidants. Molecules 15(9): 6332-48. doi: 10.3390/molecules15096332

Young K.H. 1998. Yeast two-hybrid: so many interactions, (in) so little time. Biology of Reproduction 58(2): 302-11. doi: 10.1073/pnas.110149297

Zanger U.M., Schwab M. 2013. Cytochrome P450 enzymes in drug metabolism: regulation of gene expression, enzyme activities and impact of genetic variation. Pharmacology and Therapeutics 138(1): 103-41. doi: 10.1016/j.pharmthera.2012.12.007

Zanger U.M., Turpeinen M., Klein K., Schwab M. 2008. Functional pharmacogenetics/genomics of human cytochromes P450 involved in drug biotransformation. Analytical and Bioanalytical Chemistry 392(6): 1093-108. doi: 10.1007/s00216-008-2291-6

Zhang Z.D., Burch P.E., Cooney A.J., Lanz R.B., Pereira F.A., Wu J.Q., Gibbs R.A., Weinstock G., Wheeler D.A. 2004. Genomic analysis of the nuclear 
receptor family: new insights into structure, regulation and evolution from the rat genome. Genome Research 14(4): 580-90. doi:

10.1101/gr.2160004

Zheng S.L., Chen B., Qiu X.Y., Lin K.L., Yu X.G. 2013. Three novel cytochrome P450 genes identified in the marine polychaete Perinereis nuntia and their transcriptional response to xenobiotics. Aquatic Toxicology 134: 1122. doi: 10.1016/j.aquatox.2013.02.009

Zhou C., Verma S., Blumberg B. 2009. The steroid and xenobiotic receptor (SXR), beyond xenobiotic metabolism. Nuclear Receptor Signal 7: e001. doi: $10.1621 / \mathrm{nrs} .07001$ 


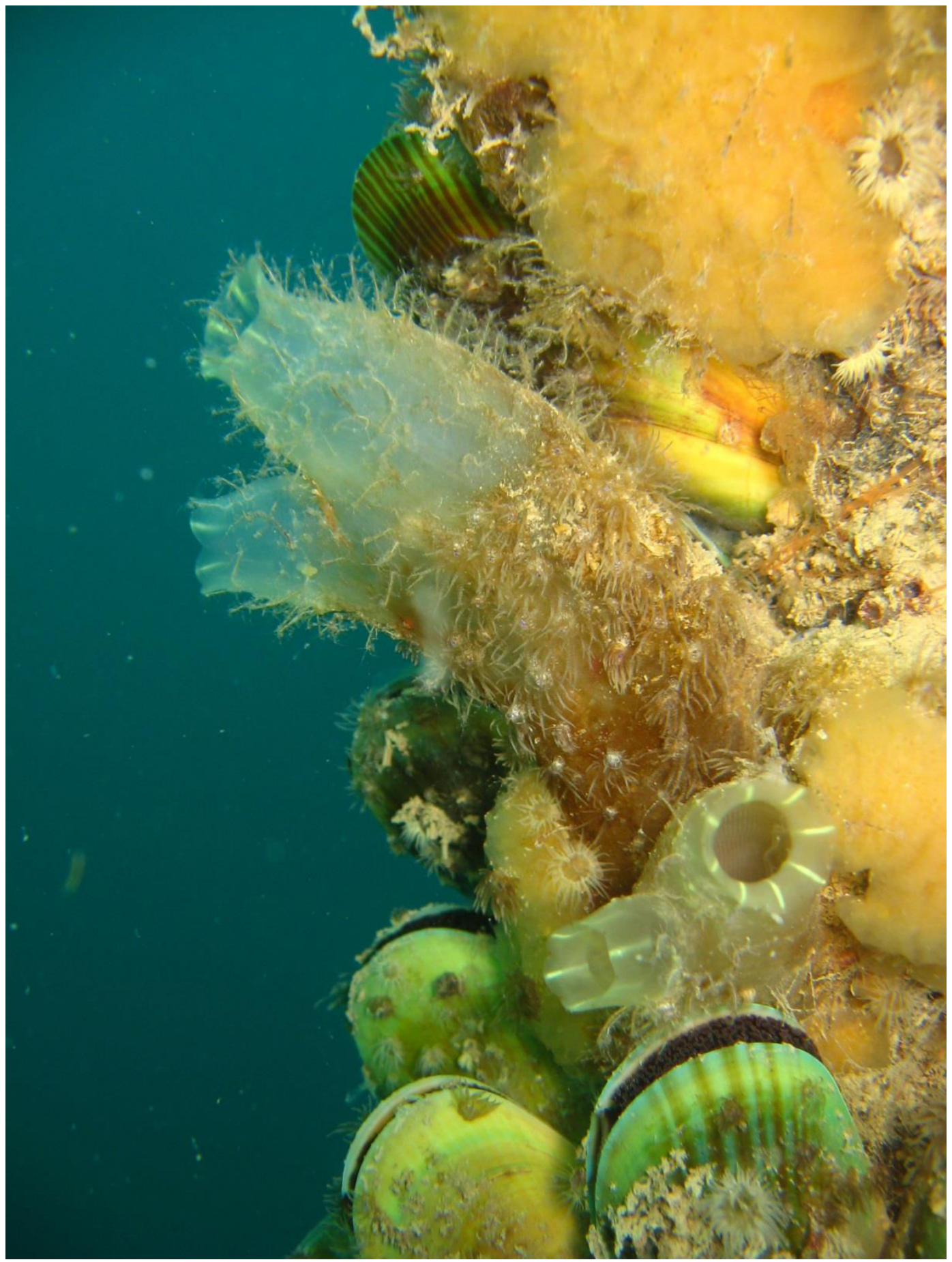

Ciona intestinalis from Lyttelton Harbour, New Zealand.

Photograph by Dr Chris Woods, National Institute of Water and Atmospheric Research, Christchurch, New Zealand. 


\section{Part One}

\section{Detection of adaptive evolution in tunicate xenobiotic receptors using molecular tools}




\section{Transcripts of tunicate orthologues of the pregnane $X$ receptor: characterisation and natural variation}

\subsection{Introduction}

Tunicates feed by filtering small organisms from seawater and therefore can be exposed to dietary xenobiotics at relatively high concentrations (Sekiguchi et al. 2001; Echevarria et al. 2012; Roje-Busatto \& Ujević 2014). Dietary xenobiotics that are toxic to tunicates (i.e. increase mortality/morbidity and/or reduce reproductive output) are likely to act as agents of natural selective pressures. Ligand-binding domains (LBDs) of xenobiotic receptors may evolve to include these compounds in their repertoire of potential ligands (Fidler et al. 2012). Evidence of adaptive evolution has been reported within LBD sequences of vertebrate xenobiotic receptors. For example, vertebrate pregnane $\mathrm{X}$ receptor (PXR) displays greater inter-taxa variation in LBD sequences than is typical of nuclear receptors (NRs), along with some evidence of positive selection within the LBD (Moore et al. 2002; Zhang et al. 2004; Krasowski et al. 2005a, b). Tunicate xenobiotic receptors are orthologues to vertebrate vitamin $D$ receptor (VDR), constitutive androstane receptor (CAR) and PXR, hence abbreviated VDR/PXR. The LBD of Ciona intestinalis VDR/PXRa has a low sequence identity when compared to vertebrate VDR, CAR and PXR (17-27\% sequence identity). In contrast, the DNA-binding domain (DBD) is more conserved (60$68 \%$ sequence identity; Table 2.1 ). It has been speculated that such inter-taxa PXR LBD sequence differences may reflect adaptive evolutionary changes enhancing binding of exogenous dietary xenobiotics typically encountered by an organism (Moore et al. 2002; Zhang et al. 2004). 
Table 2.1 Sequence identities of xenobiotic receptor orthologues.

Sequence identities of Ciona intestinalis vitamin $D$ receptor/pregnane $X$ receptor $\alpha(C N D R / P X R \alpha)$ compared to the vertebrate pregnane $X$ receptor (PXR), the vitamin D receptor (VDR) and constitutive androstane receptor (CAR). Table modified from Ekins et al. (2008).

\begin{tabular}{|c|c|c|}
\hline \multirow[t]{2}{*}{ Receptor } & \multicolumn{2}{|c|}{$\%$ Identity to Ciona intestinalis VDR/PXRa } \\
\hline & DNA-binding domain & Ligand-binding domain \\
\hline Human PXR & 61.8 & 22.5 \\
\hline Mouse PXR & 60.3 & 21.5 \\
\hline Chicken PXR & 63.2 & 23.7 \\
\hline Xenopus PXR & 64.7 & 20.3 \\
\hline Fugu PXR & 67.6 & 19.8 \\
\hline Human VDR & 67.6 & 17.1 \\
\hline Zebrafish VDR & 70.6 & 21.8 \\
\hline Sea lamprey VDR & 73.5 & 20.8 \\
\hline Human CAR & 60.3 & 26.8 \\
\hline Mouse CAR & 55.9 & 23.2 \\
\hline
\end{tabular}

Characterising the effects of natural selection at the molecular level is one of the major challenges of evolutionary genomics. In general, variation at the genetic level can affect the function of proteins, which can lead to cellular modifications, organismal phenotypes and reduced fitness (Dalziel et al. 2009). Thus, protein adaptations are characterised by 'footprints' in the genome which can be identified in genomic sequence data and analysed using a wide range of statistical methods (Biswas \& Akey 2006). McDonald and Kreitman (1991) suggested to take advantage of the degeneracy of the genetic code: nonsynonymous variation (i.e. amino acid changing) is supposed to reflect the action of natural selection, whereas synonymous changes are seen as a neutral reference. For coding sequences, comparisons between the number of nonsynonymous substitutions per non-synonymous site $\left(d_{N}\right)$ to synonymous substitutions per synonymous site $\left(d_{S}\right)$ provide a good measure of the strength and character of selection (Jensen et al. 2007). Using divergence-based maximum likelihood analyses, positive selection was detected in a number of highly variable sites in Mu-class glutathione transferases, a class of vertebrate detoxification enzymes (Ivarsson et al. 2003). These putatively selected 
changes were shown to lead to differences in protein function. This is consistent with the hypothesis that functional diversification underlies rapid amino acid evolution in detoxification genes (Norrgard et al. 2006).

Previous suggestions of positive selection acting on the LBD coding sequences of PXR, and its orthologues, were based on sequence comparisons between vertebrate taxa (Zhang et al. 2004; Krasowski et al. 2005a). To date, interspecies studies have shown significant differences in the xenobiotic response between humans and rodents which may be explained by differences in the pharmacology of mammalian PXR (Blumberg et al. 1998; Kliewer et al. 1998; Jones et al. 2000; LeCluyse 2001; Tirona et al. 2004). The aims of this chapter were to investigate intra-species sequence variation in VDR/PXR LBD coding sequences of two tunicates, $C$. intestinalis and Botryllus schlosseri, and to identify evidence of both adaptive evolution and functionally significant allelic variations.

\subsection{Materials and methods}

\subsubsection{Tunicate collection and tissue sampling}

Ciona intestinalis adults ( $>60 \mathrm{~mm}$ length, $\mathrm{n}=30$ ) were collected from four New Zealand South Island locations: (i) Nelson marina (41\%15'32.64"S, 173¹6'55.53"E, $n=18$ ); (ii) Lyttelton Harbour (43³6'38.63"S, 17242'14.35"E, $\mathrm{n}=3$ ); (iii) Pelorus Sound (41'12'54.87"S, 17352'46.91"E, $\mathrm{n}=6$ ) and; (iv) Queen Charlotte Sound (41'12'31.73"S, 174'17'59.91"E, n = 3). New Zealand Botryllus schlosseri colonies $(n=30)$ were collected from two locations: Nelson marina $(n=23)$ and Lyttelton Harbour $(n=7)$. Botryllus schlosseri colonies $(n=$ 10) from the eastern Mediterranean Coast were collected from a single location: Michmoret Beach, Ruppin Academic Centre, Israel (32²4'31.85"N, $\left.34^{\circ} 52^{\prime} 05.37 " \mathrm{E}\right)$. The Botryllus schlosseri colonies were collected $>1 \mathrm{~m}$ apart to reduce the chances of sampling clonally related colonies. Ciona intestinalis individuals were kept overnight in sea water with traces of menthol crystals to 
anesthetise the animals to assist subsequent dissection of tissues (Hanashima et al. 2012). Dissected C. intestinalis tissues were frozen on dry ice before storage at $-70^{\circ} \mathrm{C}$. Botryllus schlosseri colonies from New Zealand waters were frozen on dry ice before storage at $-70^{\circ} \mathrm{C}$. Botryllus schlosseri colonies collected in the Mediterranean were placed in RNA stabilisation buffer (http://sfg.stanford.edu/RNAbuffer.pdf) and transported at ambient temperatures to New Zealand before long term storage at $-70^{\circ} \mathrm{C}$.

\subsubsection{Amplification and Sanger sequencing of cDNA sequences}

Total RNA was isolated from either dissected $C$. intestinalis gut tissues or $B$. schlosseri colony fragments using Trizol (Life Technologies, Carlsbad, CA, U.S.A.) following the manufacturer's protocol. First strand cDNAs were synthesised from total RNA using random hexamer primers (Transcriptor First Strand cDNA Synthesis Kit, Roche Diagnostics, Penzberg, Germany) and then used as templates in polymerase chain reactions (PCRs). Primers were designed to amplify three tunicate VDR/PXR partial coding sequences. Primers flanking the coding sequences for the predicted DBD and LBD domains of $C$. intestinalis VDR/PXRa (CNDR/PXRa, GenBank acc. no.: NM 001078379) and C. intestinalis VDR/PXRß (CNDR/PXR, NM 001044366) were designed directly from the GenBank sequences with the primer sequences shown in Appendix 1A. When this work was started, sequencing of the $B$. schlosseri genome was still in progress which complicated PCR primer design. Primers for amplifying the $B$. schlosseri VDR/PXRa coding sequence were developed using a combination of alignments of PXR orthologues sequences and $B$. schlosseri expressed sequence tag (EST) sequence data. The predicted DBD protein sequences of PXR orthologues from mouse ( $\underline{A F 031814})$, rat ( $\underline{A F 151377})$, rabbit (AF188476), human ( $\underline{\text { AF061056) }}$, frog ( $\underline{\text { AF305201) }}$, chicken ( $\underline{\text { AF276753) }}$ ) and $C$. intestinalis ( $\mathrm{NM} \mathrm{001078379}$ ) were aligned. A conserved peptide sequence, CEGCKGFFR, was identified and a redundant forward primer was designed from an alignment of the corresponding DNA sequences (Appendix 1A). The forward primer was paired with a reverse primer designed directly from two $B$. 
schlosseri EST sequences (contig-18161978, contig-18290615, A. Voskoboynik, pers. comm.) identified as encoding a VDR/PXRa orthologue (Appendix 1A). Polymerase chain reactions were carried out in $20 \mu \mathrm{l}$ final volumes containing: $10 \mu \mathrm{l}$ of SAHARA ${ }^{\mathrm{TM}}$ DNA Polymerase $2 \mathrm{x}$ Master Mix (Bioline, London, U.K.), forward and reverse primers (both $0.4 \mu \mathrm{M}$ ), $32 \mu \mathrm{g} / \mathrm{ml}$ bovine serum albumin (BSA, Sigma-Aldrich, St. Louis, MO, U.S.A.) and $2 \mu \mathrm{l}$ of template cDNA. All three partial coding sequences (CNDR/PXRa, CNDR/PXR $\beta$ and $B s V D R / P X R \alpha$ ) were amplified using the following thermocycling conditions: $94^{\circ} \mathrm{C} / 10 \mathrm{~min}$., 1 cycle; $94^{\circ} \mathrm{C} / 30 \mathrm{sec}$., $45^{\circ} \mathrm{C} / 30 \mathrm{sec}$. ramping at $0.2^{\circ} \mathrm{C} / \mathrm{sec}$. to $72^{\circ} \mathrm{C} / 2$ min., 5 cycles; $94^{\circ} \mathrm{C} / 30 \mathrm{sec}$., $55^{\circ} \mathrm{C} / 30$ sec., $72^{\circ} \mathrm{C} / 2$ min., 35 cycles; $72^{\circ} \mathrm{C} / 7$ min., $1 \mathrm{cycle} ; 15^{\circ} \mathrm{C} /$ hold. The resulting PCR products were visualised on an agarose gel and purified (Zymoclean ${ }^{\mathrm{TM}}$ Gel DNA Recovery Kit, Zymo Research, Irvine, CA, U.S.A.). Purified amplicons were ligated into pGEM-TEasy $^{\mathrm{TM}}$ (Promega, Madison, WI, U.S.A.), transformed into DH5a Escherichia coli, and plasmids purified (High Pure Plasmid Isolation Kit, Roche Diagnostics). Plasmid inserts were bi-directionally sequenced by an external contractor using a vector encoded primer pair, M13 forward and reverse (Massey Genome Service, Massey University, New Zealand). A representative sequence from a single cloned haplotype from each of the three genes was deposited in GenBank with the following accession numbers: CNDR/PXRa (1326 bp): KC561370, CNDR/PXR $\beta$ (1569bp): KC561371 and BsVDR/PXRa (1006 bp): KC561372.

\subsubsection{Protein analysis software}

Structural domains of predicted protein sequences were identified using the Simple Modular Architecture Research Tool (SMART) software (Letunic et al. 2012; http://smart.embl-heidelberg.de), the National Center for Biotechnology Information (NCBI) Conserved Domain (CD) database (Marchler-Bauer et al. 2011; http://ncbi.nlm.nih.gov/Structure/cdd/wrpsb.cgi) and the Protein Families (Pfam) database (Punta et al. 2012; http://pfam.xfam.org). 


\subsubsection{Phylogenetic analysis}

DNA-binding and ligand-binding domain sequences from predicted chordate NR1I clade proteins were aligned using ClustalW (Thompson et al. 1994) implemented in the MEGA5 package (Molecular Evolutionary Genetics Analysis software, version 5; Tamura et al. 2011). Phylogenetic analyses were performed using MEGA5 with alignment positions containing gaps/missing data eliminated from the dataset (complete deletion option). Phylogenies were inferred using the neighbour-joining algorithm with maximum composite likelihood distances and 1000 bootstrap repetitions. Sequences from domestic silkworm (Bombyx mori) ecdysone receptor (AAA87341), tick (Amblyomma americanum) ecdysone receptor (AB94566) and sea urchin (Strongylocentrotus purpuratus) ecdysone receptor/farnesoid $X$ receptor (NP 001123279) were used as outgroups.

\subsubsection{Detection of Ciona intestinalis VDR/PXR $\alpha$ and $\beta$ gene transcripts}

Quantitative PCR (qPCR) was used to detect CNDR/PXR $\alpha$ and $\beta$ transcripts in five regions of the $C$. intestinalis digestive tract. Total RNA was isolated from adult $C$. intestinalis digestive tract tissues using Trizol $^{\circledR}$ Reagent. cDNA was synthesized as described in Section 2.2.2 and quantified using a Qubit $^{\circledR}$ 2.0 Fluorometer (Life Technologies). A Ciona intestinalis cytoskeletal actin gene orthologue (GenBank acc. no.: AJ297725) was used as an internal control for cDNA synthesis. All qPCR primer pairs used are listed in Appendix 1B and were predicted to flank at least one intron based on a $C$. intestinalis genomic sequence (build 1.1, NCBI). Amplifications were performed in $20 \mu \mathrm{l}$ reactions containing: $0.4 \mu \mathrm{M}$ of both primers, $10 \mu \mathrm{iQ} \mathrm{iM}^{\mathrm{TM}}$ SYBR $^{\circledR}$ Green $2 \mathrm{x}$ SuperMix (BioRad, Hercules, CA, U.S.A.) and $2 \mu$ template cDNA. Negative control reactions, in which sterile water replaced the cDNA, were run in parallel. The qPCR thermocycling conditions used were as follows: $95^{\circ} \mathrm{C} / 2 \mathrm{~min}$., 1 cycle; $95^{\circ} \mathrm{C} / 15 \mathrm{sec}$., $60^{\circ} \mathrm{C} / 1 \mathrm{~min}$., 40 cycles (Rotor-Gene ${ }^{\mathrm{TM}} 6000$, Qiagen, Hilden, 
Germany). Amplicon homogeneity and absence of primer dimers was confirmed by melt curve analysis. Data obtained from triplicate amplifications of each template were averaged and expressed in molecules/ng cDNA using the appropriate diluted plasmid-based standard curves. Amplicons were cloned into a T-tailed vector (pGEM-T-Easy ${ }^{\mathrm{TM}}$, Promega) to provide quantifiable templates for generation of standard curves. Plasmid concentrations were measured using a Nano Photometer (Implen, Munich, Germany) and then serially diluted 1:10 in water to generate standard curves displaying a linear relationship between the cycle threshold values and log10 plasmid molecule number over the range $10^{2}-$ $10^{7}$ plasmid molecules/reaction $\left(R^{2}>0.995, M=-3.4\right.$, efficiency $\left.=0.9\right)$. The data were square root transformed and one-way analysis of variance (ANOVA) was used to study the association between different tissues and the level of gene expression using the Tukey HSD test function in Statistica 9 (StatSoft Inc, Tulsa, OK, U.S.A., 2008). Homogeneity of the variances was tested using Cochran's $C$ test and a $p$ value $<0.01$ was considered significant.

\subsubsection{Illumina ${ }^{\mathrm{TM}}$ sequencing of pooled amplicons}

Tunicate VDR/PXR coding region amplicons were generated from either gut cDNA ( $C$. intestinalis) or colony cDNA (B. schlosseri) templates using primers listed in Appendix 1A. Amplicons were purified from agarose gels (Zymoclean ${ }^{\mathrm{TM}}$ Gel DNA Recovery Kit, Zymo Research) and concentrations determined using a Nano Photometer (Implen). Amplicons were then pooled on an equimolar basis generating separate pooled mixtures for the CNDR/PXR $\alpha, C N D R / P X R \beta$ and BsVDR/PXRa amplicons. The total DNA concentration in each of the amplicon mixtures was adjusted to $2 \mathrm{ng} / \mu \mathrm{l}$ (Nano Photometer, Implen). Subsequent sequencing of the amplicon pools was carried out by an external contractor (New Zealand Genomics Ltd., Massey University, New Zealand). The three amplicon mixes were characterised using an Agilent Bioanalyzer DNA 7500 Kit (Agilent Technologies, Santa Clara, CA, U.S.A.) and the Quant-iT ${ }^{\mathrm{TM}}$ dsDNA HS Assay Kit (Life Technologies) before pooling into a single mixture containing all three amplicons. The mixture was sheared by nebulisation and sequenced 
using the Illumina ${ }^{\mathrm{TM}}$ TruSeq DNA Library Preparation Kit in combination with the Illumina $^{\mathrm{TM}}$ MiSeq System (Illumina ${ }^{\mathrm{TM}}$, San Diego, CA, U.S.A.) producing pairedend reads with a mean length of ca. $150 \mathrm{bp}$. The resulting data was quality controlled using conventional procedures: (i) the Burrows-Wheeler Alignment (BWA) tool (Li \& Durbin 2010) was used to map raw sequence files against the PhiX genome and all matching reads were removed; (ii) adapter removal was performed using the fastx-clipper programme implemented in the ea-utils suite (version 1.1.2-318; Aronesty 2013) and; (iii) to remove any other potential contamination the reads were screened against a set of genome sequences (e.g. E. coli, Illumina ${ }^{\mathrm{TM}}$ adapters and the cloning vector pFosill-2) using fastQscreen (Babraham Bioinformatics 2013a). Sequence quality statistics were calculated using fastQC (Babraham Bioinformatics 2013b) and SolexaQA (Cox et al. 2010). The remaining sequences were aligned against the reference sequences for each of the three amplicons in paired-end and single-end mode using the BWA tool.

\subsubsection{Identification and analysis of polymorphisms in pooled amplicon sequence data}

The Illumina ${ }^{\mathrm{TM}}$ MiSeq generated sequence data for the CNDR/PXRa, CNDR/PXR $\beta$ and BsVDR/PXRa amplicons was examined for variants using VarScan (Koboldt et al. 2009) with default settings. Both single nucleotide polymorphism (SNP) and indel variant positions were identified for each amplicon using minimum 'cut-off' frequencies between 0.01-0.2, in increments of 0.01 . Variants identified at a minimum 'cut-off' frequency of 0.02 were entered into the NCBI dbSNP database (http://ncbi.nlm.nih.gov/SNP/snp_viewTable.cgi?type=contact\&handle=CAW_E T). Specific SNPs can be identified within the reference sequences of each gene (CNDR/PXRa: KC561370, CNDR/PXRß: KC561371 and BsVDR/PXRa: KC561372) by string searches using 25 nucleotides 5' and 3' to each SNP listed within the dbSNP database. 
Numbers of transitions, transversions and the transitional to transversional ratios $(\mathrm{Ti} / \mathrm{TV})$ per site were calculated using the Tamura-Nei model as implemented in the MEGA 5 software package (Tamura \& Nei 1993). Three population genetic parameters (Watterson's $\theta$, Tajima's $D\left(D_{T}\right)$ and $\pi$ ) were estimated using the sliding window approach implemented in the PoPoolation software (Kofler et al. 2011). Since $D_{T}$ is sensitive to variation in coverage, in part due to sequencing errors, $D_{T}$ was estimated by subsampling all reads to coverage of 1000 bases using a minimum count of two and a minimum quality of 20. This analysis only allows for relative comparison among the study populations and not for direct comparisons with other studies because $D_{T}$ depends on coverage and window size. Values for all population statistics were calculated in non-overlapping $50 \mathrm{bp}$ windows across each gene.

Non-synonymous and synonymous SNPs identified at a minimum variant frequency of 0.02 were mapped onto each of the three reference sequences (KC561370-72). Regional pairwise calculations (reference sequence vs. variant sequence) were used to calculate $d_{N} / d_{S}$ ratios in a sliding window as implemented in JCoDA (Steinway et al. 2010). Using the Yang and Nielsen (2000) substitution model, $d_{N} / d_{S}$ ratios were calculated with a window size of 200 codons and an increment jump of 25 codons.

\subsection{Results}

\subsubsection{Determination of tunicate VDR/PXR $\alpha$ and $\beta$ partial coding sequences}

Partial coding sequences from the two $C$. intestinalis VDR/PXR orthologues (CNDR/PXR a and CNDR/PXR $\beta$ ) and the B. schlosseri VDR/PXRa orthologue (BsVDR/PXRa) were amplified from appropriate cDNA templates (C. intestinalis: gut cDNA; $B$. schlosseri: total colony $\mathrm{cDNA}$ ) using the primer pairs listed in Appendix 1. 
The amplified sequences were confirmed as genuine VDR/PXR orthologues using: (i) the Basic Local Alignment Search Tool (BLAST) to find annotated GenBank entries and; (ii) phylogenetic analyses using alignments of the corresponding predicted protein sequences. In nucleotide BLAST searches (BLASTN) of GenBank (nucleotide database, no taxonomic restrictions) the CNDR/PXRa sequence ( $\underline{\text { KC561370) }}$ ) displayed 99\% identity (E value $=0$ ) with coordinates 431-1756 of the previously reported CNDR/PXRa sequence (NM 001078379). This sequence had been derived from the $C$. intestinalis genome sequence, in combination with strong EST support (AB210742; NCBI Gene ID: 778791; Ensembl transcript: ENSCINT00000031407). The BLASTN searches of GenBank (nucleotide database) using the CNDR/PXR $\beta$ sequence $(\underline{K C 561371})$ as the query revealed high sequence identity with sections of a previously reported $C$. intestinalis VDR/PXR $\beta$ sequence (NM 001044366; NCBI Gene ID: 751590; Ensembl transcript: ENSCINT00000030933) derived from the C. intestinalis genome sequence in combination with some EST support. Examination of the $\mathrm{KC561371/NM} 001044366$ alignments revealed regions of near identity interspersed with non-aligned regions. The most parsimonious interpretation of these alignments is that KC561371 and NM 001044366 are derived from splice variant transcripts from the same locus.

Both BLASTN and translated nucleotide BLAST (TBLASTN) searches of GenBank (nucleotide database, no taxonomic restrictions) using the putative BsVDR/PXRa partial coding sequence ( $\underline{\text { KC561372) }}$ identified CNDR/PXRa (NM 001078379) as the most homologous sequence on the GenBank nucleotide database (BLASTN: identity $=70 \%$ over coordinates $1214-1477$ of $\underline{\text { NM 001078379, }}$ E value $=9 e-25 ;$ TBLASTN: identity $=52 \%, E$ value $=3 e-94)$. Additionally, BLASTN searches of a $B$. schlosseri EST database (http://octopus.obs-vlfr.fr/public/botryllus/blast_botryllus.php) provided EST support for the BsVDR/PXRa ( $\underline{\text { KC561372 }}$ ) sequence (BLASTN: identity $=98 \%$ over coordinates 783-1788 of Bot_oas_2431, E value $=0.0$ ).

The orthologous relationships of the three tunicate amplified cDNA sequences were also examined by phylogenetic analysis based on alignments of conserved sections of protein sequences. Predicted protein sequences were derived from $\underline{\mathrm{KC561370}}$ (421 residues), $\underline{\mathrm{KC561371}}$ (480 residues) and 
KC561372 (333 residues). The putative DBD and LBD domains were predicted using three different methods (Figure 2.1, Appendix 2). The derived phylogeny placed the three tunicate sequences in the same NR clade as the vertebrate VDR (NR111), PXR (NR1/2) and CAR (NR1/3) genes supporting their designation as VDR/PXR orthologues (Figure 2.2). 
A

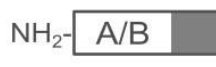

C

$\mathrm{D}$

$\mathrm{F}-\mathrm{COOH}$

DNA-binding domain

Ligand-binding domain

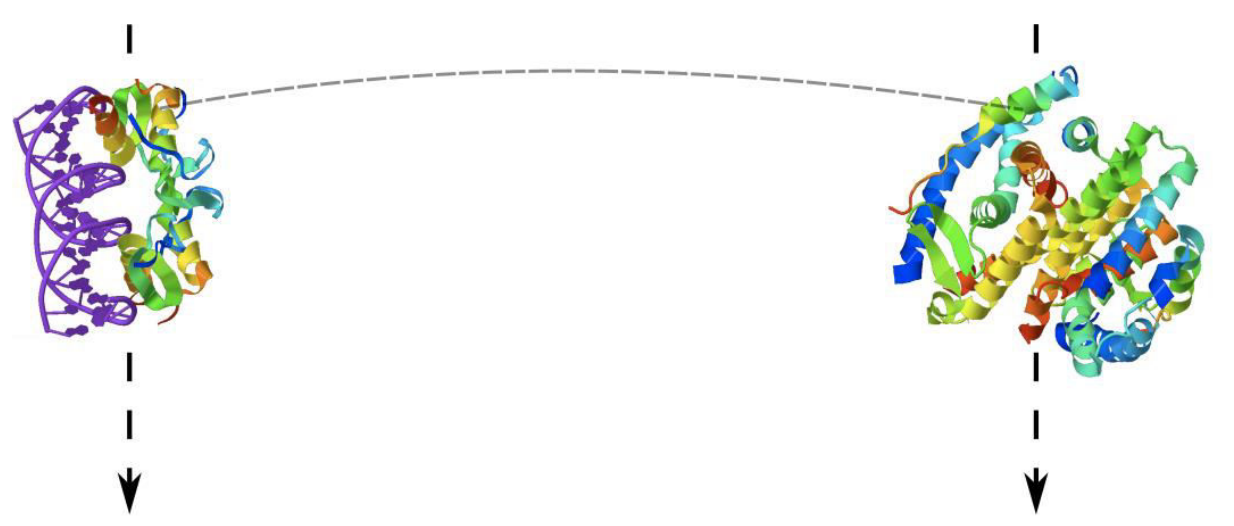

B

CNDR/PXRa
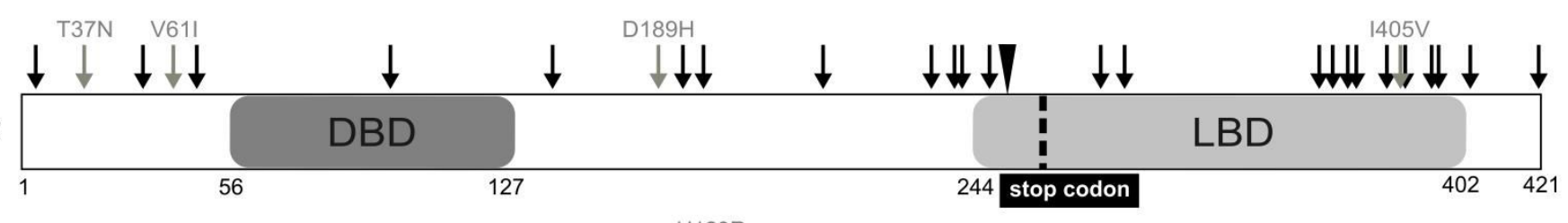

CINDR/PXR $\beta$

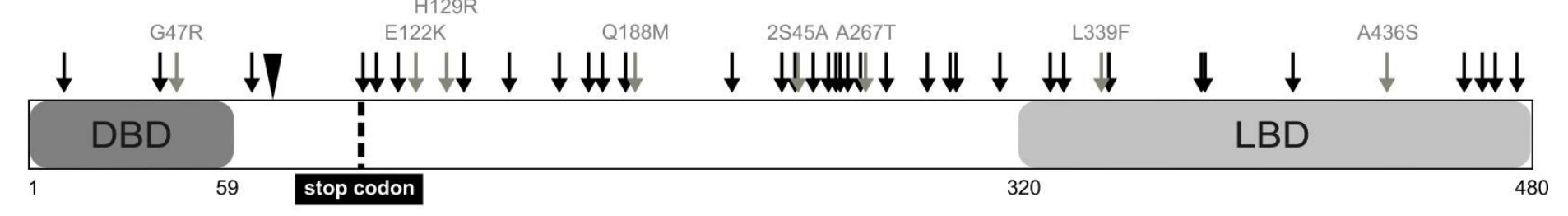

BsVDR/PXRa

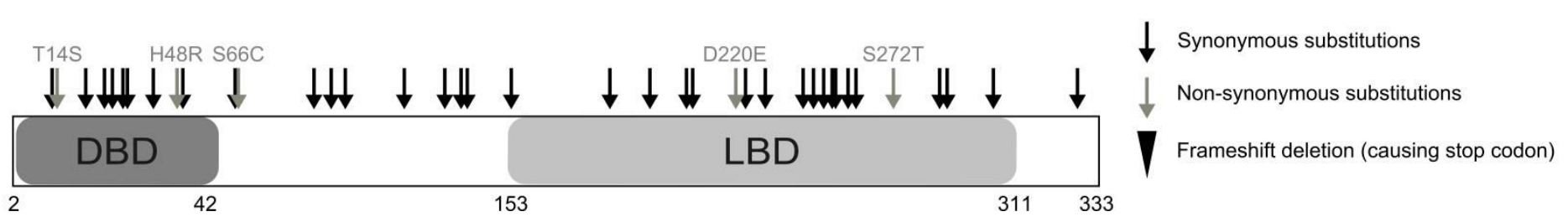

Figure 2.1 Genetic diversity within the primary structures of the predicted tunicate xenobiotic receptor proteins. 
Figure 2.1 (cont.) (A) Schematic diagram of the generic nuclear receptor structure with conserved domains indicated: $N$-terminal $A / B$ domain $(A / B)$, DNA-binding domain (DBD; C), flexible hinge region (D), ligand-binding domain (LBD; E) and C-terminal activation domain (F). (B) Schematic primary structures of the predicted Ciona intestinalis vitamin $\mathrm{D}$ receptor/pregnane $\mathrm{X}$ receptor a $(C \mathrm{NDR} / \mathrm{PXR \alpha})$, C. intestinalis VDR/PXR $\beta$ and Botryllus schlosseri VDR/PXRa proteins with residue/codon numbers following the corresponding GenBank entries: CNDR/PXRa: KC561370; CNDR/PXRB: KC561371; BsVDR/PXRa: KC561372. The DBDs (dark grey) and LBDs (light grey) predicted by the Simple Modular Architecture Research Tool (SMART; Letunic et al. 2012) are highlighted. Approximate locations of single nucleotide polymorphisms (SNPs) in the corresponding nucleotide sequences are indicated by arrows (synonymous: black; non-synonymous: grey). Alternative residues associated with nonsynonymous SNPs are indicated above the grey arrows. The approximate locations of one base pair indel variants (black triangle) are indicated along with the position of the first stop codon generated 3 ' to the indel-associated frameshift (dashed line). 


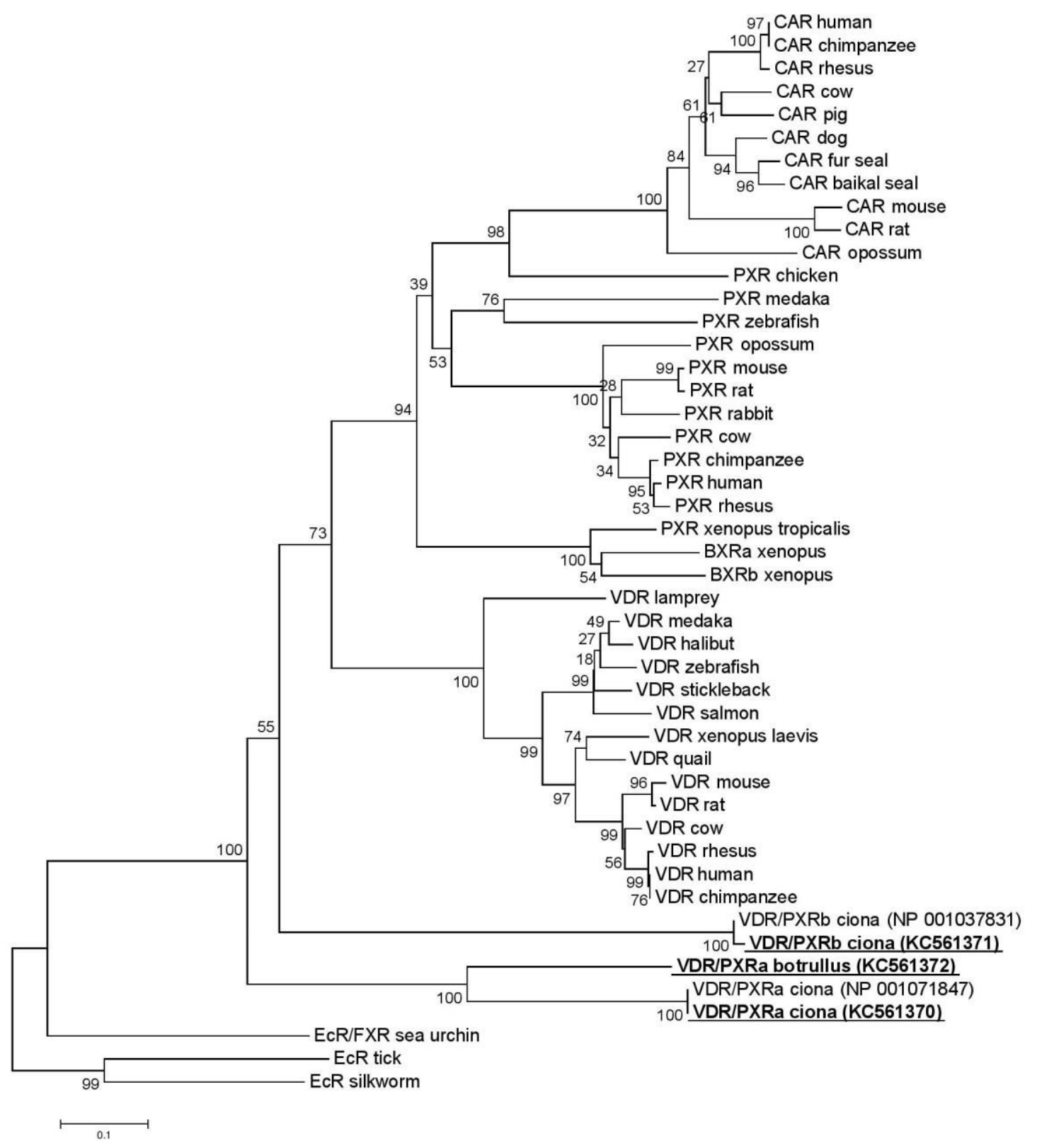

Figure 2.2 Molecular phylogeny of nuclear receptors including the three tunicate xenobiotic receptors investigated in this study.

Predicted DNA-binding domain (DBD) and ligand-binding domain (LBD) sequences from a range of metazoan nuclear receptors (NRs) were aligned and used to generate neighbour-joining trees. Bootstrap values were calculated from 1000 replicates. The sequence alignment used, along with corresponding GenBank accession numbers, is shown in Appendix 3. The three tunicate NR sequences generated in this study are indicated in underlined bold text. 


\subsubsection{Detection of VDR/PXR $\alpha$ and $\beta$ transcripts in the Ciona intestinalis digestive tract}

Both conventional PCR and quantitative PCR (qPCR) were used to detect $C N D R / P X R \alpha$ and $\beta$ transcripts in five sections of the $C$. intestinalis gut (Figure 2.3). A Ciona intestinalis cytoskeletal actin gene orthologue (GenBank acc. no.: AJ297725) was used as an internal control for both RNA integrity and CDNA synthesis. For all three $C$. intestinalis transcripts investigated, PCR primer pairs were designed to flank at least one intron sequence to ensure that amplicons generated from any contaminating genomic DNA (gDNA) could be distinguished from those amplified from $c D N A$, on the basis of their differing lengths (Appendix 1B). Using conventional PCR and amplicon visualisation on agarose gels, CNDR/PXRa, CNDR/PXR $\beta$ and Ciactin transcripts were detected in the five regions of the digestive tract with no indication of gDNA contamination as indicated by bands of the expected sizes on agarose gels (Appendix 4). Given the non-quantitative nature of conventional PCR, qPCR was used to determine transcript levels in total RNA extracted from five digestive tract regions. The estimated concentrations of the three transcripts (CNDR/PXRa, CNDR/PXR $\beta$ and Ciactin) were calculated as molecules/ng cDNA, based on plasmid standards, and grouped into five concentration ranges (Figure 2.3). Although transcripts for all three genes were detected in all five sections of the $C$. intestinalis gut, the lower part of the digestive tract appeared to have higher transcript levels for all three genes $(p<0.01$, Figure 2.3, Appendix 5). 


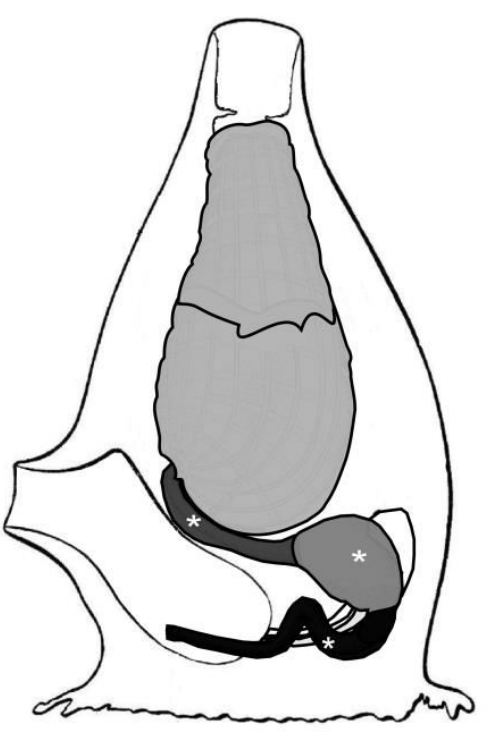

CIVDR/PXRa

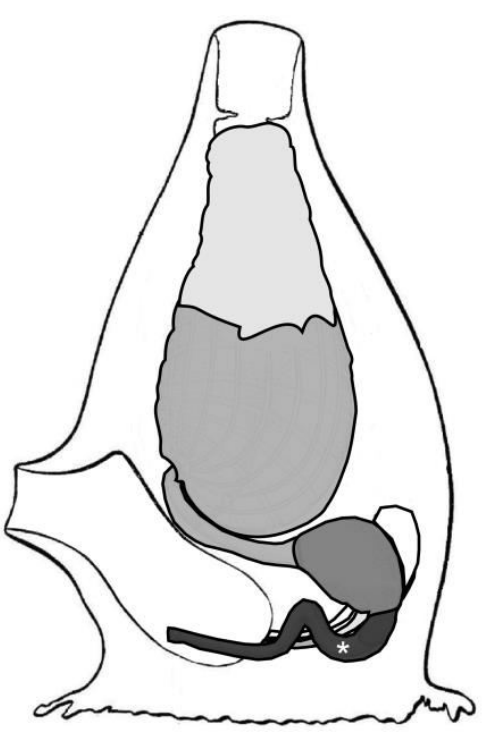

CIVDR/PXR $\beta$

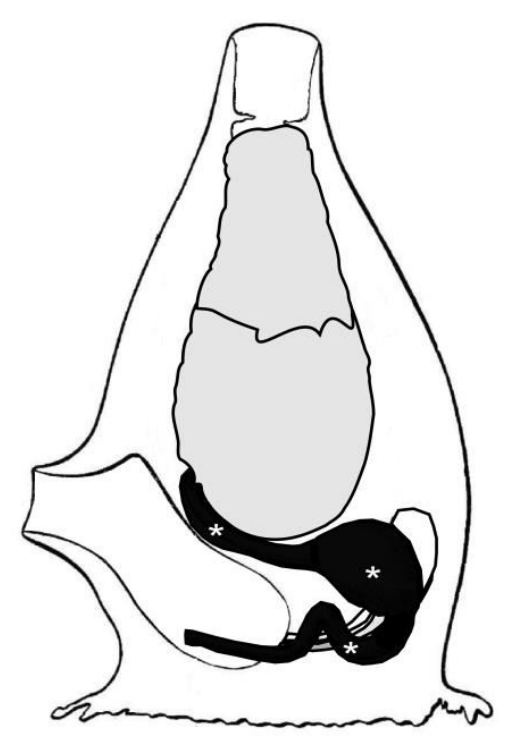

mRNA molecules/ ng cDNA

$<1,000$

$<2,000$

$<4,000$

$<8,000$

$\square>10,000$

Figure 2.3 Schematic summary of gene transcripts in gut regions of Ciona intestinalis including two xenobiotic receptors investigated in this study.

Schematic summary of Ciona intestinalis vitamin $\mathrm{D}$ receptor/pregnane $\mathrm{X}$ receptor $\alpha$ (CNDR/PXR $)$, C. intestinalis VDR/PXR $\beta$ (CNDR/PXR $\beta$ ) and $C$. intestinalis actin (Ciactin) transcript levels in five gut regions as determined using quantitative polymerase chain reaction. Estimated numbers of mRNA molecules/ng cDNA were averaged from triplicate amplification reactions. Significant differences $(p$ $<0.01)$ in gene expression between tissues are indicated $\left({ }^{*}\right)$. 


\subsubsection{Sequence polymorphisms detected in tunicate VDR/PXR amplicons}

Allelic variation in the three tunicate VDR/PXR coding sequences was assessed by sequencing pooled amplicons derived from 30 individual $C$. intestinalis animals (CNDR/PXR $\alpha$ and CNDR/PXR $\beta$ ) and 30 B. schlosseri colonies $(B s V D R / P X R \alpha)$ using the Illumina ${ }^{\mathrm{TM}}$ MiSeq System. After filtering and quality control, the paired-end sequences were mapped onto the three reference sequences (Appendix 6). Sequence coverage at each reference sequence nucleotide position was calculated up to a maximum coverage of 40,000 fold. The lowest coverage was 19,374 fold at position 127 of CNDR/PXRa (KC561370) and the average coverage was 39,454 fold (Appendix 7).

The sequence data were examined for sequence polymorphisms. Given the significant error rates associated with the Illumina ${ }^{\mathrm{TM}}$ MiSeq System (Harismendy et al. 2009; Nakamura et al. 2011), consideration was given to the minimum variant frequency necessary for a polymorphism to be a genuine polymorphism rather than a technical artefact. As expected, the number of polymorphisms predicted in all three amplicon sequence pools increased as the minimum frequency for acceptance was reduced from 0.2 to 0.01 in 0.01 decrements (Appendix 8). As the $C$. intestinalis derived amplicon pools were generated from 30 diploid animals, the minimum expected frequency for a genuine polymorphism would be 1/60 (ca. 1.7\%). Experimental errors inherent in the quantification and pooling of amplicons might result in a genuine variant being present in the data-set at a frequency $<1.7 \%$. Based on these assumptions, a value of $0.02(2 \%)$ was selected as the minimum frequency for polymorphisms in the amplicon data-set to be considered genuine (Appendix 8). Although applying a 'cut off' value of 0.02 carries the risk of excluding genuine variation, such exclusion was considered preferable to inclusion of false variants which may have occurred if the minimum variant frequency was set lower. The coordinates of the SNPs and indels identified are shown schematically in Figure 2.1, and can be obtained from Appendix 8 and from the GenBank dbSNP entries (Section 2.2.7). 
The estimated SNP numbers were as follows: CNDR/PXRa ( $\underline{\text { KC561370, }} 1326$ bp): 28 SNPs, 2.11 SNPs/100 bp; CNDR/PXR $($ KC561371, 1569 bp): 44 SNPs, 2.8 SNPs/100 bp and BsVDR/PXRa (KC561372, 1006 bp): 39 SNPs, 3.88 SNPs/100 bp (Table 2.2). Most SNPs were transitions (Ti): CNDR/PXRa: 78\%, CNDR/PXRB: 71\%, BsVDR/PXRa: 80\%, with transition/transversion rate ratios (Ti/Tv) of 3.7 (CNDR/PXRa), 2.4 (CNDR/PXRß) and 3.9 (BsVDR/PXRa; Appendix 9). The majority of SNPs were synonymous: CNDR/PXRa: $86 \%$, CNDR/PXRB: $70 \%$ and BsVDR/PXRa: $87 \%$ (Table 2.2, Figure 2.1). Mean nucleotide diversity $(\pi)$ and expected population mutation rate (Watterson's Theta; $\left.\theta_{\mathrm{w}}\right)$ were estimated for each of the coding sequences using sliding window calculations as outlined in Section 2.2.7. Tajima's $D\left(D_{T}\right)$ values were negative for all three coding sequences indicating purifying selection (Table 2.2). Regional pairwise calculations were performed via sliding window calculation of the ratio of non-synonymous substitutions per non-synonymous site $\left(d_{N}\right)$ to synonymous substitutions per synonymous site $\left(d_{S}\right)$ which were $<0.2$ across all regions of the three amplicons (Table 2.2). 
Table 2.2 Summary statistics for polymorphisms detected in tunicate xenobiotic receptor orthologues.

Polymorphisms are listed for partial coding sequences of the Ciona intestinalis vitamin $D$ receptor/pregnane $X$ receptor $\alpha$ (CNDR/PXRa), C. intestinalis VDR/PXR $\beta$ (CNDR/PXR $\beta)$ and Botryllus schlosseri VDR/PXRa (BsVDR/PXRa). Standard deviations (SD) are shown in brackets.

\begin{tabular}{|c|c|c|c|c|c|c|c|c|c|c|}
\hline & $\begin{array}{l}\text { Length } \\
\text { (bp) }\end{array}$ & $\mathbf{T}_{\mathrm{SNPS}}$ & $\begin{array}{l}1 \mathrm{bp} \\
\text { indel }\end{array}$ & $\mathbf{f}_{\text {SNPs }}$ & Syn $_{\text {SNPs }}$ & NonSyn $_{\text {SNPs }}$ & $\mathbf{d}_{N} / \mathbf{d}_{S}$ & $\pi( \pm$ SD) & $\theta_{w}( \pm S D)$ & $D_{T}( \pm S D)$ \\
\hline CNDR/PXR $\alpha$ & 1326 & 28 & 1 & 2.11 & $24(86 \%)$ & $4(14 \%)$ & 0.055 & $0.007(0.006)$ & $0.076(0.015)$ & $-3.481(0.305)$ \\
\hline$C N D R / P X R \beta$ & 1569 & 44 & 1 & 3.19 & $21(70 \%)$ & $9(30 \%)$ & 0.083 & $0.009(0.007)$ & $0.075(0.011)$ & $-3.343(0.362)$ \\
\hline$B s \vee D R / P X R \alpha$ & 1006 & 39 & & 3.87 & 34 (87\%) & $5(13 \%)$ & 0.105 & $0.013(0.009)$ & $0.081(0.014)$ & $-3.176(0.456)$ \\
\hline
\end{tabular}

Abbreviations: SNP, single nucleotide polymorphism; $T_{S N P s}$, total SNP number; bp, base pair; $f_{S N P s}$, SNP frequency (SNP/100 bp); Syn $n_{S N P s}$, synonymous

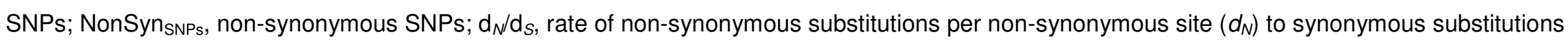
per synonymous site $\left(d_{S}\right)$ calculated using sliding window analysis (Steinway et al. 2010); $\pi$, mean pairwise nucleotide diversity (Nei \& Gojobori 1986); $\theta_{\mathrm{w}}$, expected population mutation rate (Watterson 1975); $\mathrm{D}_{\mathrm{T}}$, Tajima's D (Tajima 1989). 


\subsubsection{Frameshift polymorphisms in the Ciona intestinalis VDR/PXR coding sequences}

Single base indel polymorphisms were identified in both the CNDR/PXRa (+/coordinate 834 of $\underline{\mathrm{KC} 561370})$ and CNDR/PXRß (+/- coordinate 235 of KC561371) coding sequences (Figure 2.1, Table 2.2). The frequencies of these variants within the pooled amplicon sequence dataset were $8.4 \%$ and $2.1 \%$, respectively. Alleles carrying these one base pair frameshifts were predicted to encode VDR/PXR proteins that consist of a DBD but lacking a LBD (Figure 2.1). In support of the reliability of these indel polymorphisms, the CNDR/PXRa one base pair indel was also found in parallel investigations in which amplicons were first cloned into pGemT-easy vectors and then sequenced using Sanger sequencing (data not shown). No indels were detected in the BsVDR/PXR $\alpha$ amplicon pool when applying the minimum variant frequency 'cut off' value of 0.02 (Figure 2.1, Table 2.2, Appendix 8). However, at a 'cut off' minimum variant frequency of 0.01 additional indels were found in all three tunicate VDR/PXR coding sequences and it is possible that some of these are genuine (Appendix 8).

\subsubsection{Haplotype diversity in two Botryllus schlosseri populations from different geographical locations}

Haplotype diversity of the $B$. schlosseri VDR/PXRa partial coding sequence was compared between populations in New Zealand (Nelson Marina) and on the eastern Mediterranean coast (Michmoret Beach, Israel). To allow complete VDR/PXRa haplotype sequences to be identified with confidence, sequences were generated from cloned amplicons using Sanger sequencing. Botryllus schlosseri VDR/PXRa amplicons were amplified from cDNA templates from ten randomly selected colonies from both geographical locations and ligated into a T-tailed cloning vector before transformation into $E$.coli. Plasmids were purified from three independent $E$. coli colonies corresponding to each ligation/amplicon and their $B$. schlosseri VDR/PXRa inserts sequenced. 
Fifteen distinct haplotypes were found amongst the 30 sequences sampled from the Mediterranean population with nine haplotypes found in the 30 sequences from the New Zealand population. Consistent with this result, the SNP frequency was higher in the Mediterranean population (42 SNPs/1006 bp; 4.17 SNPs/100 bp) than in the New Zealand population (33 SNPs/1006 bp; 3.28 SNPs/100 bp, Appendix 10) with this difference being statistically significant (two-sample Kolmogorov-Smirnov test, $p=0.01$ ). In the Mediterranean sample, $24 \%(10 / 42)$ of the SNPs were non-synonymous with $11 \%(4 / 33)$ nonsynonymous in the New Zealand sample (Appendix 10). Most of the SNPS (85\%) found in New Zealand were also present in the Mediterranean population, while $69 \%$ of SNPs found in the Mediterranean were also present in the New Zealand population (Appendix 10). Despite the shared SNPs, no complete haplotypes were shared between the Mediterranean and New Zealand populations.

\subsection{Discussion}

\subsubsection{Determination of tunicate VDR/PXR $\alpha$ and $\beta$ sequences}

This chapter confirms the majority of the $C$. intestinalis VDR/PXRa coding sequence previously inferred from combined analyses of $C$. intestinalis genomic and EST sequence data (Dehal et al. 2002; Ekins et al. 2008). In contrast, the amplified $C$. intestinalis VDR/PXR $\beta$ sequences had apparent insertions of 21 and 106 codons when aligned with the $C$. intestinalis VDR/PXR $\beta$ sequence (NM 001044366) that was inferred from genomic and EST sequences. Given the limited EST support for the NM 001044366 sequence, its accuracy must be regarded with caution. The most conservative approach is to regard the CNDR/PXR $\beta$ sequence amplified in this chapter ( $\underline{\text { KC561371) }}$ ) and the NM 001044366 as splice variants. The closest homologue of BsVDR/PXRa was $C I V D R / P X R a$, suggesting that the VDR/PXRa orthologue is also present in $B$. schlosseri. This is supported by the recent release of the $B$. schlosseri 
genome (Voskoboynik et al. 2013), which contains two genomic contigs encoding VDR/PXRa.

Protein sequences predicted from the three tunicate sequences contained distinct DBDs and LBDs (Figure 2.1), thus conforming to the generic NR structure (Marchler-Bauer et al. 2011; Letunic et al. 2012; Punta et al. 2012). Although all three protein domain prediction programmes returned consistent results, these in silico predictions are based on databases consisting of proteins with known structural domains/sequences (Schultz et al. 1998; Lubec et al. 2005; Letunic et al. 2012). In the case of NRs, databases are largely composed of typical NRs (e.g. steroid hormone receptors), which have highly selective LBDs that bind a structurally limited range of ligands (Zhang et al. 2004; Krasowski et al. 2005b). As the LBD of PXR orthologues is expected to be both larger and more flexible than is typical of NR LBDs, the predicted tunicate VDR/PXR LBDs need to be treated with caution (Watkins et al. 2001; Watkins et al. 2003; Chrencik et al. 2005). To better assess the reliability of the VDR/PXR LBD software predictions, the predicted human PXR LBD was compared with the LBD coordinates that had been determined experimentally. Watkins et al. (2001) concluded from empirical crystallography data that the human PXR LBD was formed by residues 142-431. In contrast, the human PXR (NM 003889) LBD domain was considerably smaller according to software-based predictions by SMART (residues 245-404), NCBI CD (residues 236-428) and Pfam (residues 223-426). All three programmes underestimated the size of the human PXR LBD, most noticeably at the N-terminal boundary. Applying an N-terminal extension of approximately 100 residues to the SMART predicted CNDR/PXRa and BsVDR/PXRa LBD would place the N-terminal boundaries of these LBDs close to their respective DBDs. In contrast, there are approximately 250 residues between the SMART predicted CNDR/PXR $\beta$ DBD C-terminus and LBD N-terminus. In the absence of functional and/or structural information it is not possible to determine if the CNDR/PXR $\beta$ protein has a larger LBD than its VDR/PXR $\alpha$ paralogue. Thus, CNDR/PXR $\beta$ may be able to bind larger ligands or may simply have an extended linker region separating the DBD and LBD. 


\subsubsection{Expression of VDR/PXR $\alpha$ and $\beta$ transcripts in the Ciona intestinalis digestive tract}

Ciona intestinalis VDR/PXRa and VDR/PXR $\beta$ transcripts were detected throughout the digestive tract. These data suggested that VDR/PXR $\alpha$ expression levels may be higher in the lower part of the digestive tract. However, transcript levels of the internal control (actin) were also elevated making interpretation difficult. Nonetheless, detection of VDR/PXRa and VDR/PXR $\beta$ mRNA in all parts of the $C$. intestinalis digestive tract is consistent with functional roles as xenobiotic receptors - by analogy with the vertebrate PXR which is predominantly expressed in liver and intestine, which are both sites for xenobiotic metabolism (Xie et al. 2000; Staudinger et al. 2001).

\subsubsection{Variation in the tunicate VDR/PXR $\alpha$ and $\beta$ sequences}

The SNP frequencies found in the three tunicate VDR/PXR coding regions (approximately $3 \mathrm{SNPs} / 100 \mathrm{bp}$ ) are comparable to rates reported from tunicate genome sequencing projects: $B$. schlosseri, $C$. intestinalis, Ciona savignyi and Oikopleura dioica (Dehal et al. 2002; Small et al. 2007; Caputi et al. 2008; Denoeud et al. 2010; Satou et al. 2012; Tsagkogeorga et al. 2012; Voskoboynik et al. 2013). Tunicates are known to have a high level of genetic diversity, which can be up to ten times higher than in human (1 SNP/1000 bp), zebrafish and fugu (4 SNPs/1000 bp; Wang et al. 1998; Aparicio et al. 2002; Guryev et al. 2006; Wheeler et al. 2008). These high levels of genetic diversity are consistent with a large effective population size and a high per-year mutation rate $(\mu)$, which is two to six times higher in tunicates than in vertebrates due to a short generation time (Lambert 2005; Thomas et al. 2010; Tsagkogeorga et al. 2012).

Among all identified polymorphisms, transitions (Ti) outnumbered transversions (TV) by about three to one in partial coding regions of CNDR/PXRa, CNDR/PXR $\beta$ and BsVDR/PXRa. The average transition/transversion ratios $(\mathrm{Ti} / \mathrm{TV}=3.3)$ in the three coding sequences were similar to $\mathrm{Ti} / \mathrm{Tv}$ ratios reported 
for C. savignyi (Small et al. 2007). This contrasts with Ti/Tv ratios in mammalian coding sequences $(\mathrm{Ti} / \mathrm{TV}=2)$, which can be attributed to larger biases against CpG sequences in mammalian coding sequences than in tunicate coding sequences (Moriyama \& Powell 1996; Cargill et al. 1999; Lindblad-Toh et al. 2000).

Although high SNP frequencies were found, the great majority of SNPs in all three tunicate VDR/PXR coding sequences were synonymous. Together with the negative Tajima's $D$ values $\left(D_{T}<-3\right)$ and low $d_{N} / d_{S}$ ratios $(<0.2)$, the sequence variation data indicates that tunicate VDR/PXR coding sequences are under strong purifying selection. These findings are consistent with recent population genomics analyses of $C$. intestinalis which reported a very low average $d_{N} / d_{S}$ ratio $(0.07)$ in combination with high SNP frequencies (approximately 5.7 SNPs/100 bp; Tsagkogeorga et al. 2012). This is consistent with tunicates having elevated mutation rates and large effective population sizes in combination with strong purifying selection (Kimura 1983; Lynch 2008; Tsagkogeorga et al. 2012; Berná \& Alvarez-Valin 2014).

Overall, this interpretation would be the most parsimonious explanation of the SNP types, and frequencies, detected in the tunicate VDR/PXR coding sequences. However, single amino acid changes can have significant functional consequences. Non-synonymous SNPs in the human PXR gene alter PXR protein function as measured by basal and/or induced transactivation of CYP3A promoters (Hustert et al. 2001). Similarly, two natural human CAR sequence variants were shown to alter the receptor's constitutive (ligand-independent) transactivation activity (Ikeda et al. 2005). The effects of single residue differences can be extended to inter-taxa difference. For example, differences in human and mouse PXR activation by rifampicin can be attributed to differences at a single position: human PXR Leu $\mathrm{U}_{308} /$ rat PXR Phe 305 (Tirona et al. 2004).

\subsubsection{Frameshift polymorphisms}

Both $C$. intestinalis VDR/PXR genes had at least one allele containing a singlebase deletion resulting in the predicted proteins having a DBD but lacking a 
LBD. The persistence of such frameshift alleles in natural populations, at low but significant frequencies, suggests that they are maintained by some form of balancing selection. The corresponding predicted proteins that lack LBDs seem likely to mediate constitutive, rather than ligand-activated, gene transcription. In support of this interpretation, NR variants lacking a LBD have been shown to constitutively activate transcription in vertebrates (e.g. human androgen receptor; Tepper et al. 2002; Marcias et al. 2010) and insects (e.g. Knirps; Nauber et al. 1988). Similarly, a number of NR splice variants have been described that translate into proteins lacking LBDs and which constitutively activate target genes (e.g. human estrogen receptor; Chaidarun et al. 1998). To date, no equivalent splice variants lacking a LBD have been described for vertebrate PXRs (Hustert et al. 2001). In short, the VDR/PXRa and $\beta$ frameshift alleles are likely to activate target gene transcription constitutively which may confer advantages over ligand-activated transcription in some contexts.

\subsubsection{Haplotype diversity in two Botryllus schlosseri populations from different geographical locations}

The B. schlosseri population from the eastern Mediterranean coast displayed greater VDR/PXRa genetic diversity than the New Zealand population. The lower genetic diversity of New Zealand populations, when compared to Mediterranean populations, has been noted in previous studies using microsatellite loci (Ben-Shlomo et al. 2001; Paz et al. 2003). This observation has been intrepreted as reflecting founder effects associated with introduction events to New Zealand (Ben-Shlomo et al. 2001). Consistent with this interpretation, the majority of VDR/PXRa SNPs found in the New Zealand population $(>85 \%$ ) were present in the Mediterranean population, whereas $30 \%$ of the SNPs in the Mediterranean population were unique to that population. No complete haplotype sequences were shared between the two populations. This is consistent with the high rates of genetic variation and recombination frequencies characteristic of tunicate genomes (Simmen \& Bird 2000; Satoh \& Levine 2005; Caputi et al. 2008; Satou et al. 2012; Tsagkogeorga et al. 2012). 


\subsection{Conclusion}

The mRNA sequences of two $C$. intestinalis and one $B$. schlosseri VDR/PXR orthologues have been characterised. All three corresponding proteins were predicted to have the typical NR structure, containing distinct LBDs and DBDs. The CNDR/PXR $\beta$ LBD may be larger than the CNDR/PXRa LBD, suggesting that it might accommodate larger ligands. All three transcripts harboured high rates of sequence polymorphism with evidence of purifying selection. Examination of $d_{N} / d_{S}$ ratios over an entire gene is extremely conservative and is unlikely to detect positive selection acting on a few sites (Jensen et al. 2007). It is also possible for loci to be under selection without yielding statistically significant results in tests for selection (Przeworski et al. 2005; Teshima et al. 2006). However, allelic variants that contained one base pair frameshift polymorphisms were detected in both $C$. intestinalis VDR/PXR orthologues. The resulting proteins were predicted to contain a DBD but lacking a LBD. Such VDR/PXR variants may mediate constitutive transcription of target genes. Haplotype variation of $B$. schlosseri VDR/PXRa was found to be higher in a population from the Mediterranean than New Zealand, which is consistent with founder effects during colonisation. The data presented in this chapter provide a foundation for further investigations into the molecular evolution, population genetics and function of tunicate NRs thought to be involved in the detection of marine bioactive compounds.

\subsection{References}

Aparicio S., Chapman J., Stupka E., Putnam N., Chia J., Dehal P., Christoffels A., Rash S., Hoon S., Smit A. et al. 2002. Whole-genome shotgun assembly and analysis of the genome of Fugu rubripes. Science 297(5585): 1301-10. doi: 10.1126/science.1072104

Aronesty E. 2013. Comparison of sequencing utility programs. The Open Bioinformatics Journal 7. doi: 10.2174/1875036201307010001

Babraham Bioinformatics 2013a. FastQ Screen. Accessed on: 03 June 2013, from http://bioinformatics.babraham.ac.uk/projects/fastq_screen

Babraham Bioinformatics 2013b. FastQC. Accessed on: 03 June 2013, from http://bioinformatics.babraham.ac.uk/projects/fastqc 
Ben-Shlomo R., Douek J., Rinkevich B. 2001. Heterozygote deficiency and chimerism in remote populations of a colonial ascidian from New Zealand. Marine Ecology Progress Series 209: 109-17. doi: 10.3354/meps209109

Berná L., Alvarez-Valin F. 2014. Evolutionary genomics of fast evolving tunicates. Genome Biology and Evolution. doi: 10.1093/gbe/evu122

Biswas S., Akey J.M. 2006. Genomic insights into positive selection. Trends in Genetics 22(8): 437-46. doi: 10.1016/j.tig.2006.06.005

Blumberg B., Sabbagh W., Juguilon H., Bolado J., van Meter C.M., Ono E.S., Evans R.M. 1998. SXR, a novel steroid and xenobiotic-sensing nuclear receptor. Genes and Development 12(20): 3195-205. doi: 10.1101/gad.12.20.3195

Caputi L., Borra M., Andreakis N., Biffali E., Sordino P. 2008. SNPs and Hox gene mapping in Ciona intestinalis. BMC Genomics 9. doi: 10.1186/1471-2164-9-39

Cargill M., Altshuler D., Ireland J., Sklar P., Ardlie K., Patil N., Lane C.R., Lim E.P., Kalyanaraman N., Nemesh J.et al. 1999. Characterization of singlenucleotide polymorphisms in coding regions of human genes. Nature Genetics 22(3): 231-8. doi: 10.1038/10290

Chaidarun S.S., Alexander J.M. 1998. A tumor-specific truncated estrogen receptor splice variant enhances estrogen-stimulated gene expression. Molecular Endocrinology 12(9): 1355-66. doi: 10.1210/me.12.9.1355

Chrencik J.E., Orans J., Moore L.B., Xue Y., Peng L., Collins J.L., Wisely G.B., Lambert M.H., Kliewer S.A., Redinbo M.R. 2005. Structural disorder in the complex of human pregnane $X$ receptor and the macrolide antibiotic rifampicin. Molecular Endocrinology 19(5): 1125-34. doi: 10.1210/me.2004-0346

Cox M.P., Peterson D.A., Biggs P.J. 2010. SolexaQA: At-a-glance quality assessment of Illumina second-generation sequencing data. BMC Bioinformatics 11. doi: 10.1186/1471-2105-11-485

Dalziel A.C., Rogers S.M., Schulte P.M. 2009. Linking genotypes to phenotypes and fitness: how mechanistic biology can inform molecular ecology. Molecular Ecology 18(24): 4997-5017. doi: 10.1111/j.1365294X.2009.04427.x

Dehal P., Satou Y., Campbell R.K., Chapman J., Degnan B., De Tomaso A., Davidson B., Di Gregorio A., Gelpke M., Goodstein D.M.et al. 2002. The draft genome of Ciona intestinalis: insights into chordate and vertebrate origins. Science 298(5601): 2157-67. doi: 10.1126/science.1080049

Denoeud F., Henriet S., Mungpakdee S., Aury J.-M., Da Silva C., Brinkmann H., Mikhaleva J., Olsen L.C., Jubin C., Canestro C.et al. 2010. Plasticity of animal genome architecture unmasked by rapid evolution of a pelagic tunicate. Science 330(6009): 1381-5. doi: 10.1126/science.1194167

Echevarria M., Naar J.P., Tomas C., Pawlik J.R. 2012. Effects of Karenia brevis on clearance rates and bioaccumulation of brevetoxins in benthic suspension feeding invertebrates. Aquatic Toxicology 106: 85-94. doi: 10.1016/j.aquatox.2011.10.011

Ekins S., Reschly E.J., Hagey L.R., Krasowski M.D. 2008. Evolution of pharmacologic specificity in the pregnane $X$ receptor. BMC Evolutionary Biology 8. doi: 10.1186/1471-2148-8-103

Fidler A.E., Holland P.T., Reschly E.J., Ekins S., Krasowski M.D. 2012. Activation of a tunicate (Ciona intestinalis) xenobiotic receptor orthologue 
by both natural toxins and synthetic toxicants. Toxicon 59: 365-72. doi: 10.1016/j.toxicon.2011.12.008

Guryev V., Koudijs M.J., Berezikov E., Johnson S.L., Plasterk R.H.A., van Eeden F.J.M., Cuppen E. 2006. Genetic variation in the zebrafish. Genome Research 16(4): 491-7. doi: 10.1101/gr.4791006

Hanashima A., Ogasawara M., Nomiya Y., Sasaki T., Bao Y., Kimura S. 2012. Genomic- and protein-based approaches for connectin (titin) identification in the ascidian Ciona intestinalis. Methods 56(1): 18-24. doi: 10.1016/j.ymeth.2011.12.010

Harismendy O., Ng P.C., Strausberg R.L., Wang X., Stockwell T.B., Beeson K.Y., Schork N.J., Murray S.S., Topol E.J., Levy S.et al. 2009. Evaluation of next generation sequencing platforms for population targeted sequencing studies. Genome Biology 10(3). doi: 10.1186/gb-2009-10-3r32

Hustert E., Zibat A., Presecan-Siedel E., Eiselt R., Mueller R., Fuss C., Brehm I., Brinkmann U., Eichelbaum M., Wojnowski L.et al. 2001. Natural protein variants of pregnane $X$ receptor with altered transactivation activity toward CYP3A4. Drug Metabolism and Disposition 29(11): 14549.

Ikeda S., Kurose K., Jinno H., Sai K., Ozawa S., Hasegawa R., Komamura K., Kotake T., Morishita H., Kamakura S.et al. 2005. Functional analysis of four naturally occurring variants of human constitutive androstane receptor. Molecular Genetics and Metabolism 86(1-2): 314-9. doi: 10.1016/j.ymgme.2005.05.011

Ivarsson Y., Mackey A.J., Edalat M., Pearson W.R., Mannervik B. 2003. Identification of residues in glutathione transferase capable of driving functional diversification in evolution. A novel approach to protein redesign. Journal of Biological Chemistry 278(10): 8733-8. doi: 10.1074/jbc.M211776200

Jensen J.D., Wong A., Aquadro C.F. 2007. Approaches for identifying targets of positive selection. Trends in Genetics 23(11): 568-77. doi: 10.1016/j.tig.2007.08.009

Jones S.A., Moore L.B., Shenk J.L., Wisely G.B., Hamilton G.A., McKee D.D., Tomkinson N.C.O., LeCluyse E.L., Lambert M.H., Willson T.M.et al. 2000. The pregnane $X$ receptor: a promiscuous xenobiotic receptor that has diverged during evolution. Molecular Endocrinology 14(1): 27-39. doi: $10.1210 / \mathrm{me} \cdot 14.1 .27$

Kimura M. 1983. The neutral theory of molecular evolution. first ed. Cambridge, Cambridge University Press. 208-33 p.

Kliewer S.A., Moore J.T., Wade L., Staudinger J.L., Watson M.A., Jones S.A., McKee D.D., Oliver B.B., Willson T.M., Zetterstrom R.H.et al. 1998. An orphan nuclear receptor activated by pregnanes defines a novel steroid signaling pathway. Cell 92(1): 73-82. doi: 10.1016/s00928674(00)80900-9

Koboldt D.C., Chen K., Wylie T., Larson D.E., McLellan M.D., Mardis E.R., Weinstock G.M., Wilson R.K., Ding L. 2009. VarScan: variant detection in massively parallel sequencing of individual and pooled samples. Bioinformatics 25(17): 2283-5. doi: 10.1093/bioinformatics/btp373

Kofler R., Orozco-terWengel P., De Maio N., Pandey R.V., Nolte V., Futschik A., Kosiol C., Schloetterer C. 2011. PoPoolation: a toolbox for population 
genetic analysis of next generation sequencing data from pooled individuals. PloS One 6(1). doi: 10.1371/journal.pone.0015925

Krasowski M.D., Yasuda K., Hagey L.R., Schuetz E.G. 2005a. Evolution of the pregnane $X$ receptor: adaptation to cross-species differences in biliary bile salts. Molecular Endocrinology 19(7): 1720-39. doi: 10.1210/me.2004-0427

Krasowski M.D., Yasuda K., Hagey L.R., Schuetz E.G. 2005b. Evolutionary selection across the nuclear hormone receptor superfamily with a focus on the NR1I subfamily (vitamin D, pregnane $X$ and constitutive androstane receptors). Nuclear Receptor 3: 2. doi: 10.1186/1478-1336-32

Kulman J.D., Harris J.E., Nakazawa N., Ogasawara M., Satake M., Davie E.W. 2006. Vitamin K-dependent proteins in Ciona intestinalis, a basal chordate lacking a blood coagulation cascade. PNAS 103(43): 15794-9. doi: $10.1073 /$ pnas.0607543103

Lambert G. 2005. Ecology and natural history of the protochordates. Canadian Journal of Zoology 83(1): 34-50. doi: 10.1139/z04-156

LeCluyse E.L. 2001. Pregnane $X$ receptor: molecular basis for species differences in CYP3A induction by xenobiotics. Chemico-Biological Interactions 134(3): 283-9. doi: 10.1016/s0009-2797(01)00163-6

Letunic I., Doerks T., Bork P. 2012. SMART 7: recent updates to the protein domain annotation resource. Nucleic Acids Research 40(D1): D302-D5. doi: $10.1093 /$ nar/gkr931

Li H., Durbin R. 2010. Fast and accurate long-read alignment with BurrowsWheeler transform. Bioinformatics 26(5): 589-95. doi: 10.1093/bioinformatics/btp698

Lindblad-Toh K., Winchester E., Daly M.J., Wang D.G., Hirschhorn J.N., Laviolette J.P., Ardlie K., Reich D.E., Robinson E., Sklar P.et al. 2000. Large-scale discovery and genotyping of single-nucleotide polymorphisms in the mouse. Nature Genetics 24(4): 381-6. doi: $10.1038 / 74215$

Lubec G., Afjehi-Sadat L., Yang J.W., John J.P.P. 2005. Searching for hypothetical proteins: theory and practice based upon original data and literature. Progress in Neurobiology 77(1-2): 90-127. doi: 10.1016/j.pneurobio.2005.10.001

Lynch M. 2008. The cellular, developmental and population-genetic determinants of mutation-rate evolution. Genetics 180(2): 933-43. doi: 10.1534/genetics.108.090456

Marchler-Bauer A., Lu S.N., Anderson J.B., Chitsaz F., Derbyshire M.K., DeWeese-Scott C., Fong J.H., Geer L.Y., Geer R.C., Gonzales N.R.et al. 2011. CDD: a conserved domain database for the functional annotation of proteins. Nucleic Acids Research 39: D225-D9. doi: 10.1093/nar/gkq1189

Marcias G., Erdmann E., Lapouge G., Siebert C., Barthelemy P., Duclos B., Bergerat J.-P., Ceraline J., Kurtz J.-E. 2010. Identification of novel truncated androgen receptor (AR) mutants including unreported premRNA splicing variants in the 22Rv1 hormone-refractory prostate cancer (PCa) cell line. Human Mutation 31(1): 74-80. doi: 10.1002/humu.21138 McDonald J.H., Kreitman M. 1991. Adaptive protein evolution at the Adh locus in Drosophila. Nature 351(6328): 652-4. doi:10.1038/351652a0 
Moore L.B., Maglich J.M., McKee D.D., Wisely B., Willson T.M., Kliewer S.A., Lambert M.H., Moore J.T. 2002. Pregnane X receptor (PXR), constitutive androstane receptor (CAR) and benzoate $X$ receptor (BXR) define three pharmacologically distinct classes of nuclear receptors. Molecular Endocrinology 16(5): 977-86. doi: 10.1210/me.16.5.977

Moriyama E.N., Powell J.R. 1996. Intraspecific nuclear DNA variation in Drosophila. Molecular Biology and Evolution 13(1): 261-77.

Nakamura K., Oshima T., Morimoto T., Ikeda S., Yoshikawa H., Shiwa Y., Ishikawa S., Linak M.C., Hirai A., Takahashi H.et al. 2011. Sequencespecific error profile of Illumina sequencers. Nucleic Acids Research 39(13): 16. doi: 10.1093/nar/gkr344

Nauber U., Pankratz M.J., Kienlin A., Seifert E., Klemm U., Jackle H. 1988. Abdominal segmentation of the Drosophila embryo requires a hormone receptor-like protein encoded by the gap gene knirps. Nature 336(6198): 489-92. doi: 10.1038/336489a0

Nei M., Gojobori T. 1986. Simple methods for estimating the numbers of synonymous and nonsynonymous nucleotide substitutions. Molecular Biology and Evolution 3(5): 418-26.

Norrgard M.A., Ivarsson Y., Tars K., Mannervik B. 2006. Alternative mutations of a positively selected residue elicit gain or loss of functionalities in enzyme evolution. PNAS 103(13): 4876-81. doi:

10.1073/pnas.0600849103

Paz G., Douek J., Mo C.Q., Goren M., Rinkevich B. 2003. Genetic structure of Botryllus schlosseri (Tunicata) populations from the Mediterranean coast of Israel. Marine Ecology Progress Series 250: 153-62. doi: 10.3354/meps250153

Przeworski M., Coop G., Wall J.D. 2005. The signature of positive selection on standing genetic variation. Evolution 59(11): 2312-23. doi: 10.1554/05273.1

Punta M., Coggill P.C., Eberhardt R.Y., Mistry J., Tate J., Boursnell C., Pang N., Forslund K., Ceric G., Clements J.et al. 2012. The Pfam protein families database. Nucleic Acids Research 40(D1): D290-D301. doi: 10.1093/nar/gkr1065

Roje-Busatto R., Ujević I. 2014. PSP toxins profile in ascidian Microcosmus vulgaris (Heller, 1877) after human poisoning in Croatia (Adriatic Sea). Toxicon 79(0): 28-36. doi: 10.1016/j.toxicon.2013.12.014

Satoh N., Levine M. 2005. Surfing with the tunicates into the post-genome era. Genes and Development 19(20): 2407-11. doi: 10.1101/gad.1365805

Satou Y., Shin-I T., Kohara Y., Satoh N., Chiba S. 2012. A genomic overview of short genetic variations in a basal chordate, Ciona intestinalis. BMC Genomics 13: 208. doi: 10.1186/1471-2164-13-208

Schultz J., Milpetz F., Bork P., Ponting C.P. 1998. SMART, a simple modular architecture research tool: identification of signaling domains. PNAS 95(11): 5857-64. doi: 10.1073/pnas.95.11.5857

Sekiguchi K., Sato S., Kaga S., Ogata T., Kodama M. 2001. Accumulation of paralytic shellfish poisoning toxins in bivalves and an ascidian fed on Alexandrium tamarense cells. Fisheries Science 67(2): 301-5. doi: 10.1046/j.1444-2906.2001.00228.x

Simmen M.W., Bird A. 2000. Sequence analysis of transposable elements in the sea squirt, Ciona intestinalis. Molecular Biology and Evolution 17(11): 1685-94. 
Small K.S., Brudno M., Hill M.M., Sidow A. 2007. Extreme genomic variation in a natural population. PNAS 104(13): 5698-703. doi:

10.1073/pnas.0700890104

Staudinger J.L., Goodwin B., Jones S.A., Hawkins-Brown D., MacKenzie K.I., Latour A., Liu Y.P., Klaassen C.D., Brown K.K., Reinhard J.et al. 2001. The nuclear receptor PXR is a lithocholic acid sensor that protects against liver toxicity. PNAS 98(6): 3369-74. doi:

10.1073/pnas.051551698

Steinway S.N., Dannenfelser R., Laucius C.D., Hayes J.E., Nayak S. 2010. JCoDA: a tool for detecting evolutionary selection. BMC Bioinformatics 11. doi: 10.1186/1471-2105-11-284

Tajima F. 1989. Statistical method for testing the neutral mutattion hypothesis by DNA polymorphism. Genetics 123(3): 585-95.

Tamura K., Nei M. 1993. Estimation of the number of nucleotide substitutions in the control region of mitochondrial DNA in humans and chimpanzees. Molecular Biology and Evolution 10(3): 512-26.

Tamura K., Peterson D., Peterson N., Stecher G., Nei M., Kumar S. 2011. MEGA5: molecular evolutionary genetics analysis using maximum likelihood, evolutionary distance and maximum parsimony methods. Molecular Biology and Evolution 28(10): 2731-9. doi:

10.1093/molbev/msr121

Tepper C.G., Boucher D.L., Ryan P.E., Ma A.H., Xia L.A., Lee L.F., Pretlow T.G., Kung H.J. 2002. Characterization of a novel androgen receptor mutation in a relapsed CWR22 prostate cancer xenograft and cell line. Cancer Research 62(22): 6606-14.

Teshima K.M., Coop G., Przeworski M. 2006. How reliable are empirical genomic scans for selective sweeps? Genome Research 16(6): 702-12. doi: $10.1101 /$ gr.5105206

Thomas J.A., Welch J.J., Lanfear R., Bromham L. 2010. A generation time effect on the rate of molecular evolution in invertebrates. Molecular Biology and Evolution 27(5): 1173-80. doi: 10.1093/molbev/msq009

Thompson J.D., Higgins D.G., Gibson T.J. 1994. CLUSTAL W: improving the sensitivity of progressive multiple sequence alignment through sequence weighting, position-specific gap penalties and weight matrix choice. Nucleic Acids Research 22(22): 4673-80. doi: 10.1093/nar/22.22.4673

Tirona R.G., Leake B.F., Podust L.M., Kim R.B. 2004. Identification of amino acids in rat pregnane $X$ receptor that determine species-specific activation. Molecular Pharmacology 65(1): 36-44. doi: 10.1124/mol.65.1.36

Tsagkogeorga G., Cahais V., Galtier N. 2012. The population genomics of a fast evolver: high levels of diversity, functional constraint and molecular adaptation in the tunicate Ciona intestinalis. Genome Biology and Evolution 4(8): 740-9. doi: 10.1093/gbe/evs054

Voskoboynik A., Neff N.F., Sahoo D., Newman A.M., Pushkarev D., Koh W., Passarelli B., Fan H.C., Mantalas G.L., Palmeri K.J.et al. 2013. The genome sequence of the colonial chordate, Botryllus schlosseri. eLife 2. doi: 10.7554/eLife.00569

Wang D.G., Fan J.B., Siao C.J., Berno A., Young P., Sapolsky R., Ghandour G., Perkins N., Winchester E., Spencer J.et al. 1998. Large-scale identification, mapping and genotyping of single-nucleotide 
polymorphisms in the human genome. Science 280(5366): 1077-82. doi: 10.1126/science.280.5366.1077

Watkins R.E., Wisely G.B., Moore L.B., Collins J.L., Lambert M.H., Williams S.P., Willson T.M., Kliewer S.A., Redinbo M.R. 2001. The human nuclear xenobiotic receptor PXR: structural determinants of directed promiscuity. Science 292(5525): 2329-33. doi: 10.1126/science.1060762

Watkins R.E., Maglich J.M., Moore L.B., Wisely G.B., Noble S.M., DavisSearles P.R., Lambert M.H., Kliewer S.A., Redinbo M.R. 2003. 2.1 angstrom crystal structure of human PXR in complex with the St. John's wort compound hyperforin. Biochemistry 42(6): 1430-8. doi: 10.1021/bi0268753

Watterson G.A. 1975. Number of segregating sites in genetic models without recombination. Theoretical Population Biology 7(2): 256-76. doi: 10.1016/0040-5809(75)90020-9

Wheeler D.A., Srinivasan M., Egholm M., Shen Y., Chen L., McGuire A., He W., Chen Y.-J., Makhijani V., Roth G.T.et al. 2008. The complete genome of an individual by massively parallel DNA sequencing. Nature 452(7189): 872-U5. doi: 10.1038/nature06884

Xie W., Barwick J.L., Downes M., Blumberg B., Simon C.M., Nelson M.C., Neuschwander-Tetri B.A., Bruntk E.M., Guzelian P.S., Evans R.M. 2000. Humanized xenobiotic response in mice expressing nuclear receptor SXR. Nature 406(6794): 435-9. doi: 10.1038/35019116

Yang Z.H., Nielsen R. 2000. Estimating synonymous and nonsynonymous substitution rates under realistic evolutionary models. Molecular Biology and Evolution 17(1): $32-43$.

Zhang Z.D., Burch P.E., Cooney A.J., Lanz R.B., Pereira F.A., Wu J.Q., Gibbs R.A., Weinstock G., Wheeler D.A. 2004. Genomic analysis of the nuclear receptor family: new insights into structure, regulation and evolution from the rat genome. Genome Research 14(4): 580-90. doi: 10.1101/gr.2160004 


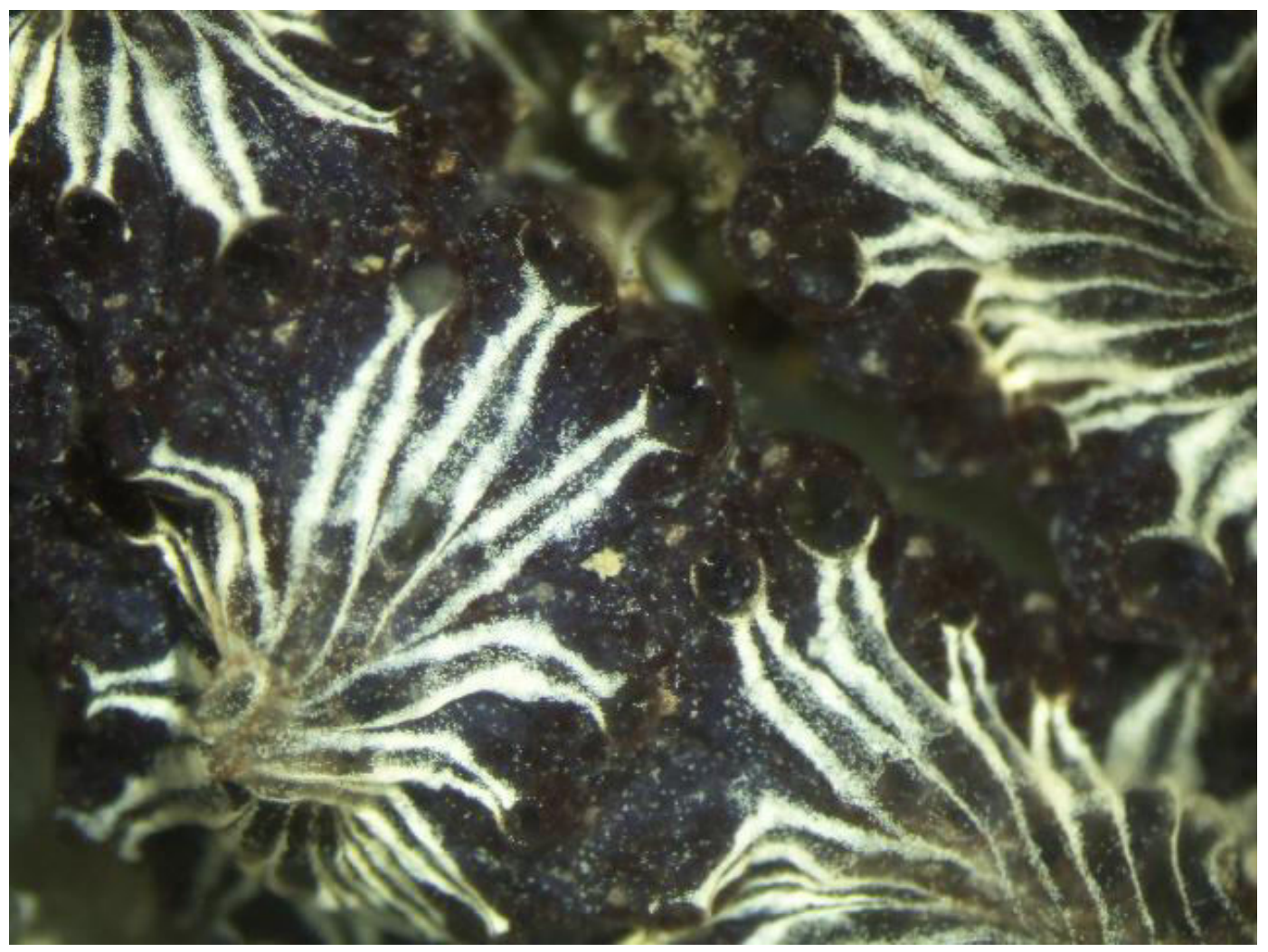

Botryllus schlosseri colonies from Nelson marina, New Zealand. Photograph provided by Prof Baruch Rinkevich, National Institute of Oceanography, Israel. 


\section{Part Two}

Functional characterisation of tunicate xenobiotic receptors and their application in yeast-based bioassays 


\section{Detection of marine microalgal biotoxins using bioassays based on functional expression of tunicate xenobiotic receptors in yeast}

\subsection{Introduction}

Marine microalgal taxa can produce highly toxic chemicals that can cause widespread poisoning within marine ecosystems. These toxic compounds may also affect human health, particularly when accumulated in filter-feeding shellfish (Wear \& Gardner 2001; Wang 2008; MacKenzie et al. 2011; Zhang et al. 2013). Over the past two decades, internationally accepted methodologies for the detection of such natural marine biotoxins have become increasingly based on chemical, rather than biological, assays (Gerssen et al. 2010; Suzuki \& Quilliam 2011; McNabb et al. 2012). While such chemistry-based detection methods are generally reliable and highly specific, they require specialised equipment and technically skilled personnel. Detailed structural knowledge regarding the targeted biotoxins is also essential, thus unknown marine biotoxins cannot be detected (Humpage et al. 2010; Nicolas et al. 2014). Although a range of in vitro bioassay tests for microalgal biotoxins has been developed (Bovee et al. 2011; Banerjee et al. 2013; Nicolas et al. 2014), the most widely-used bioassay remains the unreliable and ethically questionable mouse mortality bioassay (Botana et al. 2009; Buckland 2010; Stewart \& McLeod 2014). There is, therefore, a need for new, ethically-acceptable, simple, robust and inexpensive bioassays (Vilarino et al. 2010; Hashimoto et al. 2011; Nicolas et al. 2014).

In Chapter Two, putative xenobiotic receptors that are orthologues to vertebrate pregnane $X$ receptor (PXR) and vitamin $D$ receptor (VDR) were successfully identified in two tunicate species, Ciona intestinalis and Botryllus schlosseri (abbreviated VDR/PXR). Genomic sequence-based analyses are always 
tentative and functional data are needed for the confident assignment of these proteins as a functional xenobiotic receptor. The $C$. intestinalis VDR/PXR $\alpha$ ( $C / N D R / P X R \alpha)$ has been characterised in terms of its ligand-binding domain (LBD) mediating ligand-dependent luciferase reporter gene expression when fused to the generic GAL4 DNA-binding domain (GAL4-DBD) and expressed in a mammalian cell line (Ekins et al. 2008). Using this bioassay, three synthetic chemicals and two microalgal biotoxins (pectenotoxin-2 (PTX-2) and okadaic acid) were identified as CNDR/PXRa agonists and a common ligand pharmacophore was defined (Reschly et al. 2007; Ekins et al. 2008; Fidler et al. 2012). Collectively, these studies established that the CNDR/PXRa receptor has ligand-binding characteristics consistent with a natural role in detecting marine xenobiotics, including microalgal biotoxins. This makes it a potential sensor element for use in bioassays (Fidler et al. 2012).

In this chapter, I report the development of recombinant yeast (Saccharomyces cerevisiae) strains that express the $C$. intestinalis and B. schlosseri VDR/PXRa LBD in combination with the GAL4-DBD and a generic transcription activation domain (VP16-AD). These recombinant proteins mediate ligand-dependent expression of the easily assayed lacZ reporter gene to facilitate detection of probable ligands for tunicate VDR/PXRa receptors. The bioassay strains were tested with four synthetic toxicants and five natural toxins including three microalgal biotoxins (pectenotoxin-11 (PTX-11), okadaic acid and portimine) and two freshwater cyanobacterial toxins (anatoxin-A and microcystin-RR). 


\subsection{Materials and methods}

\subsubsection{Compounds tested in the yeast bioassay}

Four synthetic compounds ( $n$-butyl- $p$-aminobenzoate, carbamazepine, $p$ aminobenzoic acid and bisphenol-A) tested in the yeast bioassay were purchased from Sigma-Aldrich (St. Louis, MO, U.S.A.; Table 3.1). The algal toxin okadaic acid was purchased from Sapphire Bioscience (Waterloo, Australia), microcystin-RR from DHI Lab Products (Horsholm, Denmark) and anatoxin-A was purchased from the National Research Council Canada (Ottawa, Canada). Pectenotoxin-11 (MacKenzie et al. 2013) and portimine (Selwood et al. 2013) were sourced from within the Cawthron Institute (Nelson, New Zealand; Table 3.2). Chemical structures are given in Figure 3.1. All compounds were dissolved in analytical grade ethanol (Merck, Whitehouse Station, NJ, U.S.A.) to form stock solutions. Serial dilutions were added to the yeast bioassay media at a final ethanol concentration of $1 \%(\mathrm{v} / \mathrm{v}$; Bovee et al. 2004). Solubility of test compounds in ethanol was confirmed using the PubChem database (http://pubchem.ncbi.nlm.nih.gov/) and ethanol was chosen as the preferred standard solvent for consistency across all assays. In some cases, due to the limited solubility of some compounds, chemicals were also dissolved in dimethyl sulfoxide (DMSO, Sigma-Aldrich) and tested in the yeast bioassays (Appendix 11). 

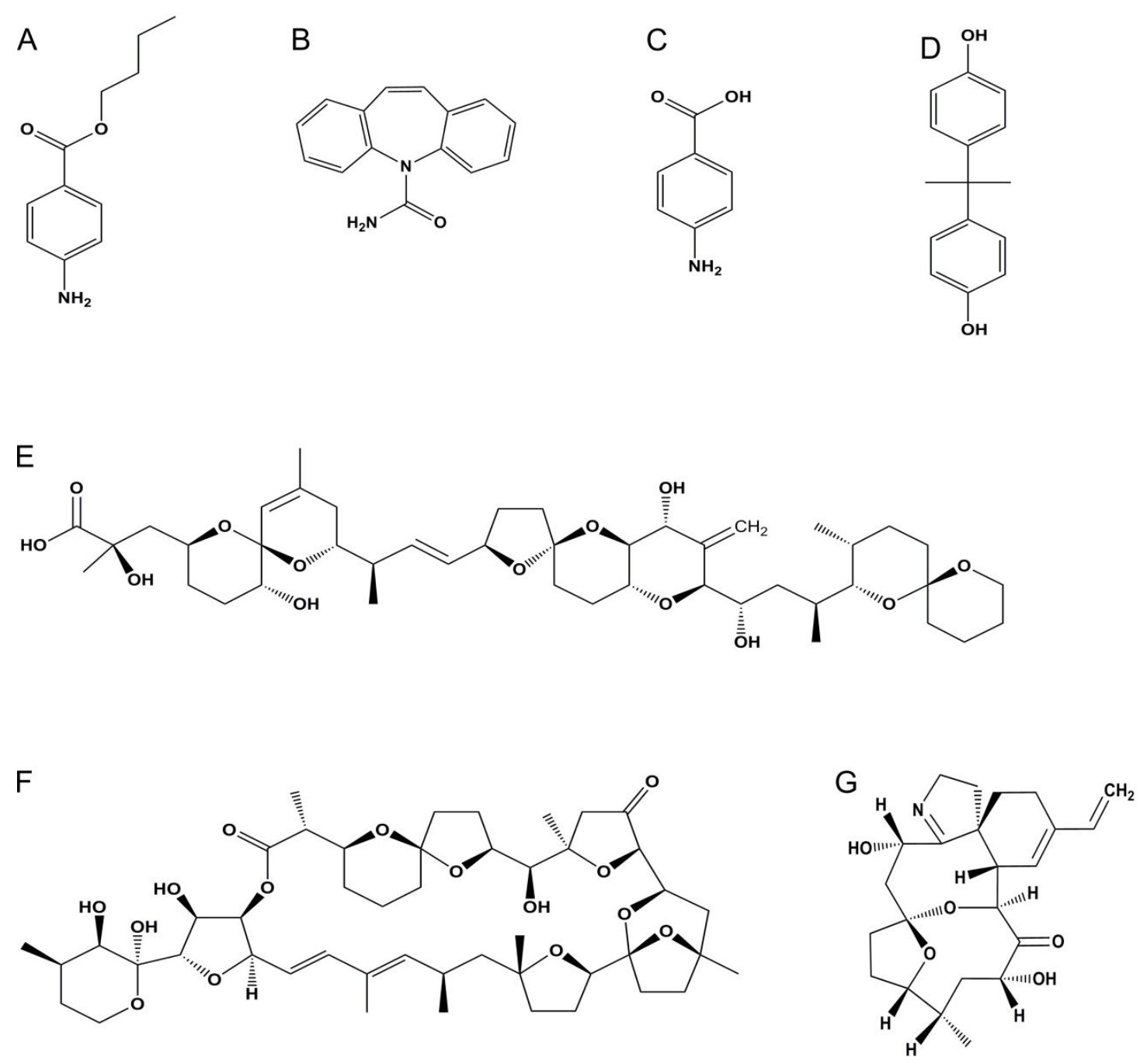

H

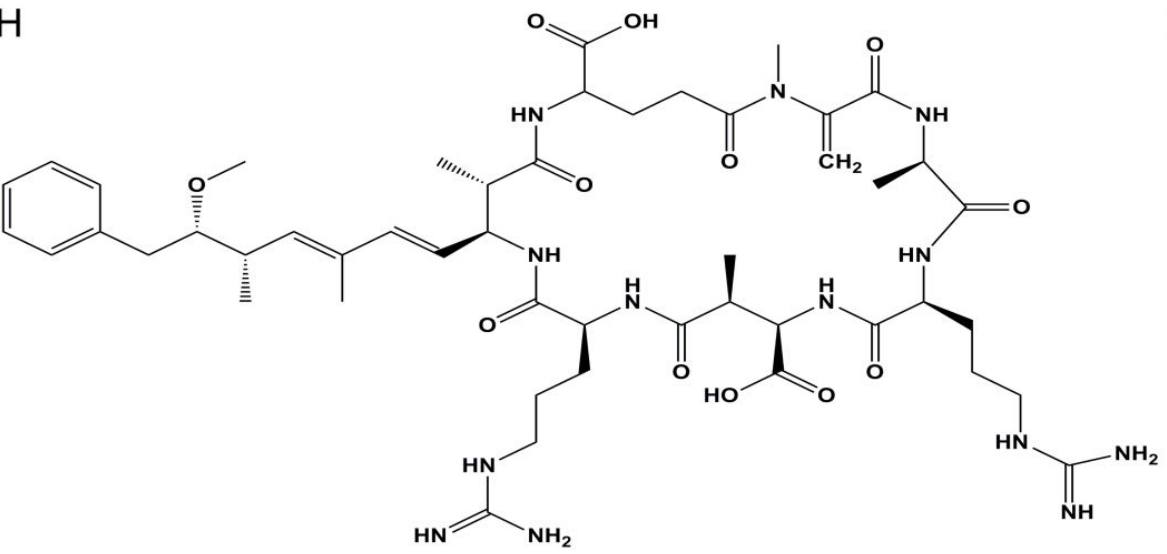

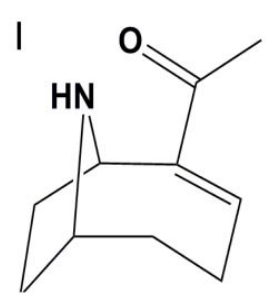

Figure 3.1 Structures of four synthetic chemicals and five algal toxins.

The following chemicals were tested in tunicate VDR/PXRa LBD-based yeast bioassays: (A) $n$-butyl-p-amino-benzoate, (B) carbamazepine, (C) $p$-aminobenzoic acid, (D) bisphenol-A, (E) okadaic acid, (F) pectenotoxin-11, (G) portimine, (H) microcystin-RR and (I) anatoxin-A. 


\subsubsection{Media}

All media ingredients were purchased from Sigma-Aldrich unless stated otherwise. Minimal media (MM) was prepared following Routledge and Sumpter (1996) and Beresford et al. (2000). However, three amino acids (L-histidine, Ltyrosine and L-phenylalanine) were omitted. Minimal media (100 $\mathrm{mM} \mathrm{KH}_{2} \mathrm{PO}_{4}$, $15 \mathrm{mM}\left(\mathrm{NH}_{4}\right)_{2} \mathrm{SO}_{4}$ (Merck), $75 \mathrm{mM} \mathrm{KOH}, 1.7 \mathrm{mM} \mathrm{MgSO}{ }_{4}, 0.38 \mathrm{mM}$ L-leucine, $0.11 \mathrm{mM}$ L-arginine, $0.13 \mathrm{mM}$ L-methionine, $0.23 \mathrm{mM}$ L-isoleucine, $0.16 \mathrm{mM} \mathrm{L}$ lysine, $0.68 \mathrm{mM}$ L-glutamic acid, $1.3 \mathrm{mM} \mathrm{L}$-valine, $3.6 \mathrm{mM}$ L-serine and $0.37 \mathrm{mM}$ adenine) had its $\mathrm{pH}$ adjusted to 7.1 using $5 \mathrm{M} \mathrm{KOH}$ before $\mathrm{Fe}_{2}\left(\mathrm{SO}_{4}\right)_{3}$ solution was added to a final concentration of $2 \mu \mathrm{M}$. Minimal media was autoclaved $\left(121^{\circ} \mathrm{C}, 15 \mathrm{~min}\right)$ and stored at room temperature. Growth media (GM) was prepared by supplementing MM as follows: $2 \%(\mathrm{w} / \mathrm{v}) \mathrm{D}(+)$ glucose, $0.89 \mathrm{mM}$ adenine, $0.17 \mathrm{mM}$ uracil, $1.52 \mathrm{mM}$ L-threonine, $0.71 \mathrm{mM}$ L-aspartic acid, $1 / 200$ volume of $200 x$ vitamin stock solution (1.7 $\mu \mathrm{M}$ pantothenic acid, $11 \mu \mathrm{M}$ myo-inositol, $1.9 \mu \mathrm{M}$ pyridoxine $\mathrm{HCl}$ and $1.2 \mu \mathrm{M}$ thiamine $\mathrm{HCl}, 0.082 \mu \mathrm{M}$ biotin, filter-sterilised) and 1/1000 volume of $1000 x$ trace elements solution (8.08 $\mu \mathrm{M} \mathrm{H}_{3} \mathrm{BO}_{3}$ (Merck), $0.25 \mu \mathrm{M} \mathrm{CuSO}{ }_{4} .5 \mathrm{H}_{2} \mathrm{O}$ (Merck), $0.6 \mu \mathrm{M} \mathrm{KI}, 2.64 \mu \mathrm{M}$ $\mathrm{MnO}_{4} \mathrm{~S} .4 \mathrm{H}_{2} \mathrm{O}$ and $\left.0.97 \mu \mathrm{M} \quad \mathrm{Na}_{2} \mathrm{MoO}_{4} .2 \mathrm{H}_{2} \mathrm{O}, 2.47 \mu \mathrm{M} \quad \mathrm{ZnSO}_{4} .7 \mathrm{H}_{2} \mathrm{O}\right)$. For corresponding solid media, agar was added at $2 \%(\mathrm{w} / \mathrm{v})$.

\subsubsection{Generation of plasmid constructs}

All the plasmid constructs used in this study were derived from the 7.3 kilo base (kb) yeast expression vector pGBKT7 (Catalogue no. 630443, Clontech Laboratories Inc., Mountain View, CA, U.S.A.). The pGBKT7 vector encodes 147 residues of the GAL4-DBD located 5' to an adjacent multiple cloning site (MCS; Figure 3.2). Transcription of GAL4-DBD fusion genes is initiated from a constitutive alcohol dehydrogenase $1(A D H 1)$ promoter $\left(P_{A D H 1}\right)$ and transcription is terminated by an $A D H 1$ transcription termination signal $\left(T_{A D H 1}\right)$. Selection for the pGBKT7 plasmid is mediated by kanamycin resistance $\left(K^{R} n^{R}\right)$ in Escherichia coli and by a nutritional marker gene (TRP1) in yeast. All 
expression plasmids produced in this thesis were generated following the same general procedure. Amplicons were generated from appropriate templates, either plasmid (pM2-GAL4-CNDR/PXRa, C. intestinalis VDR/PXRa) or cDNA (B. schlosseri VDR/PXRa) with the following reaction conditions: (i) pGAL4.VP16, pGAL4.CIBD, pGAL4.CILBDS1.VP16 and pGAL4.CILBD.VP16: 1x BioMix (BioLine, London, U.K.); $94^{\circ} \mathrm{C} / 2$ min., x1 cycle; $94^{\circ} \mathrm{C} / 30 \mathrm{sec}$., $55^{\circ} \mathrm{C} / 30 \mathrm{sec}$, $72^{\circ} \mathrm{C} / 90 \mathrm{sec}$. $x 35$ cycles; $72^{\circ} \mathrm{C} / 5 \mathrm{~min}$., x1 cycle; $15^{\circ} \mathrm{C}$ hold and; (ii) pGAL4.BsLBD and pGAL4.BSLBD.VP16: 1x Sahara Mix (BioLine); $94^{\circ} \mathrm{C} / 10 \mathrm{~min}$., $x 1$ cycle; $94^{\circ} \mathrm{C} / 30 \mathrm{sec}$, $45^{\circ} \mathrm{C} / 30 \mathrm{sec}$, ramp $45^{\circ} \mathrm{C}-72^{\circ} \mathrm{C}$ at $0.2^{\circ} \mathrm{C} / \mathrm{sec} ., 72^{\circ} \mathrm{C} / 2 \mathrm{~min}$., $x 5$ cycles; $94^{\circ} \mathrm{C} / 30 \mathrm{sec}$., $55^{\circ} \mathrm{C} / 30 \mathrm{sec}$., $72^{\circ} \mathrm{C} / 2 \mathrm{~min}$, x35 cycles; $72^{\circ} \mathrm{C} / 7 \mathrm{~min}$., $\mathrm{x} 1$ cycle; $15^{\circ} \mathrm{C}$ hold. Primers used for each construct are summarised in Appendix 12 and each included the restriction enzyme cleavage sites required for sub-cloning. Because amplicon ends are often refractory to restriction enzyme digestion the amplicons were first separated in agarose gels. Amplicons were gel-extracted (Zymoclean ${ }^{\mathrm{TM}}$ Gel DNA Recovery Kit, Zymo Research, Irvine, CA, U.S.A.) and ligated into the T-tailed cloning vector pGemT-easy (Promega, Madison, WI, U.S.A.) before transformation into competent DH5a E. coli cells. Plasmids were purified using a commercial kit (High Pure Plasmid Isolation Kit, Roche Diagnostics, Penzberg, Germany) and screened for appropriate inserts by Sanger sequencing (Massey Genome Service, Palmerston North, New Zealand). Subsequently, the plasmid inserts were removed by digestion with an appropriate pair of restriction enzymes (Roche Diagnostics; Appendix 12). The receiving expression plasmid, either pGBKT7 or its derivative of pGAL4.VP16 (Appendix 12), was double digested with an appropriate pair of restriction enzymes and ligated with the digested amplicon using T4 DNA ligase (Roche Diagnostics). Plasmids were transformed into DH5a E. coli and transformants selected on luria broth (LB) agar containing kanamycin $(25 \mu \mathrm{g} / \mathrm{ml})$. Plasmids were purified and insert identity and junction sequences confirmed by using sequencing primers flanking the MCS of pGBKT7: 5'-GCCGTCACAGATAGATTGGC-3' (anneals at coordinates 10771096 of pGBKT7) and 5'-TTCGCCCGGAATTAGCTTGG-3' (anneals at coordinates 1416-1397 of pGBKT7). For many of the plasmid constructs the base vector used (pGAL4.VP16) was derived from pGBKT7 and encodes 68 residues of a generic activation domain from the herpes simplex virus protein 16 
(VP16; Louvion et al. 1993; Appendix 12 and 13). The fusion proteins encoded by the various constructs used in this study are shown schematically in Figure 3.2 and more details are given in Appendix 13.

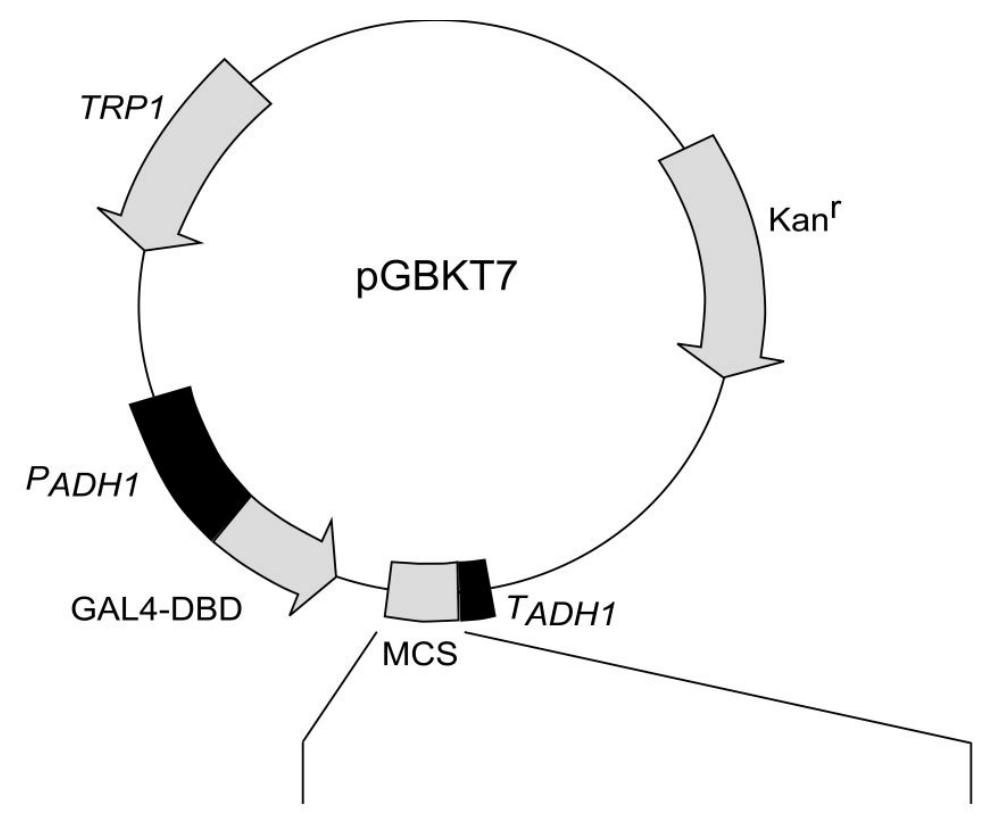

pGAL4

GAL4-DBD

pGAL4.VP16

GAL4-DBD VP16-AD

pGAL4.CiLBD

GAL4-DBD 221

pGAL4.BsLBD

GAL4-DBD 393

pGAL4.CiLBD $\triangle 31 . V P 16$

GAL4-DBD $221 \quad 501$

pGAL4.CiLBD.VP16

GAL4-DBD $221 \quad 532$

pGAL4.BsLBD.VP16

GAL4-DBD 39 Bs 343

Figure 3.2 Schematic of fusion genes/chimeric proteins generated in this study.

The base vector used for expression was pGBKT7 (Catalogue No. 630443, Clontech). Constitutive transcription of fusion genes is driven by the alcohol dehydrogenase 1 $(A D H 1)$ promoter $\left(P_{A D H 1}\right)$ and terminated by the $A D H 1$ transcription termination signal $\left(T_{A D H 1}\right)$. The pGBKT7 vector replicates autonomously in both Escherichia coli and Saccharomyces cerevisiae and carries a kanamycin resistance $\left(\mathrm{Kan}^{R}\right)$ gene for 
selection in E. coli and the nutritional marker gene TRP1 for selection in trp1 yeast strains. The pGBKT7 vector encodes 147 residues of the GAL4 DNA-binding domain (GAL4-DBD) adjacent to a multiple cloning site (MCS). The amino acid residue numbering for the Ciona intestinalis vitamin $\mathrm{D}$ receptor/pregnane $\mathrm{X}$ receptor $\alpha$ (CNDR/PXRa) and Botryllus schlosseri VDR/PXRa (BsVDR/PXRa) ligand-binding domains (LBDs) corresponds to that of the predicted proteins of NP 001071847 and $\underline{\text { AHB39790 }}$, respectively. Some of the plasmid constructs encode 68 residues of a generic activation domain (AD) from the herpes simplex virus protein 16 (VP16; Louvion et al. 1993). Further details of the cloning procedure used and the corresponding chimeric protein sequences are given in Appendix 12 and 13.

\subsubsection{Yeast transformation}

The S. cerevisiae host strain PJ69-4A (MATa, trp1-901, leu2-3 112, ura3-52, his3-200, gal4A, gal80A, GAL2-ADE2, LYS2::GAL1-HIS3, met2::GAL7-lacZ; James et al. 1996) was transformed using the lithium acetate method (Gietz \& Schiestl 2007) with tryptophan prototrophic transformants selected on solid selection media lacking tryptophan $(2 \%(w / v)$ agar, $0.67 \%(w / v)$ Difco yeast nitrogen base without amino acids, 2\% (w/v) acid-hydrolysed casamino acids, $2 \%(\mathrm{w} / \mathrm{v}) \mathrm{D}(+)$ glucose, $120 \mu \mathrm{g} / \mathrm{ml}$ adenine, $20 \mu \mathrm{g} / \mathrm{ml}$ uracil, $20 \mu \mathrm{g} / \mathrm{ml} \mathrm{L}-$ methionine and $20 \mu \mathrm{g} / \mathrm{ml}$ L-histidine). Single colony transformants were streaked out twice on selective media agar plates to ensure that the transformed strains used were clonal. The resulting strains were subsequently propagated on GM agar plates. The seven strains used to prepare starter stocks corresponded to each of the seven plasmids generated in this work (Figure 3.2, Appendix 12 and 13).

\subsubsection{Preparation of yeast starter stocks}

Yeast strains were grown in $50 \mathrm{ml}$ of liquid $\mathrm{GM}$ in $300 \mathrm{ml}$ conical flasks $\left(30^{\circ} \mathrm{C}\right.$, $230 \mathrm{rpm}$ ) for $24-48 \mathrm{~h}$ until reaching an $\mathrm{OD}_{620}$ of approximately 1 (Nano Photometer, Implen, Munich, Germany). Culture aliquots of $0.5 \mathrm{ml}$ were mixed 
with $0.5 \mathrm{ml}$ of $30 \%(\mathrm{v} / \mathrm{v})$ sterile glycerol (final glycerol concentration $15 \%(\mathrm{v} / \mathrm{v})$ ) and frozen on dry ice before storage at $-70^{\circ} \mathrm{C}$.

\subsubsection{Yeast bioassay procedure}

Freshly prepared GM (7 ml in $50 \mathrm{ml}$ centrifuge tubes, CentriStar ${ }^{\mathrm{TM}}$, Corning Inc., NY, U.S.A.) was inoculated with $1 \mathrm{ml}$ of 'yeast starter stock' (Section 3.2.5, final glycerol concentration $2.1 \%(\mathrm{v} / \mathrm{v}))$ and incubated $\left(30^{\circ} \mathrm{C}, 230 \mathrm{rpm}\right)$ for $24-48 \mathrm{~h}$ until the $\mathrm{OD}_{620}$ was approximately 1 (Nano Photometer, Implen). The cultures were then diluted in $\mathrm{GM}$ to $\mathrm{OD}_{620}$ of approximately 0.1 and this mixture was termed the seeded assay medium (SAM). A twelve step dilution series of chemicals to be tested was prepared in sterile $1.7 \mathrm{ml}$ microcentrifuge tubes (Eppendorf AG, Hamburg, Germany) using analytical grade ethanol as the solvent (Section 3.2.1). Aliquots $(2 \mu \mathrm{l})$ of each dilution were pipetted onto the floor of the wells of a 96-well microtitre plate (sterile, flat bottom polystyrene plates with lid, Corning Inc.). To facilitate inter-plate comparisons, each plate contained two columns of control wells (column one, rows $\mathrm{A}-\mathrm{H}$ : wells containing SAM only; column two, rows A-H: containing $2 \mu$ ethanol solvent plus SAM; Figure 3.3).

Based on published mean effective concentrations ( $\mathrm{EC}_{50}$ values) and in vivo toxicity data, a wide concentration range $(0-1 \mathrm{mM})$ of test compounds was selected for the initial bioassays. Each compound was tested at least twice in separate assays. These data were then used to identify a concentration range that allowed detection of induction of $\beta$-galactosidase enzymatic activity in the bioassay while being non-toxic to the yeast cells themselves. Using the identified concentration range, all yeast bioassays were performed in pseudotriplicates (triplicate measurements within the same assay).

Aliquots of SAM $(150 \mu \mathrm{l})$ were added to all wells and the plates shaken (600 rpm, 2 min., MSI Minishaker, IKA, Hamburg, Germany) before incubation $\left(30^{\circ} \mathrm{C}\right)$ for $24 \mathrm{~h}$ to allow ligand-dependent activation of lac $\mathrm{Z}$ gene expression (De Boever et al. 2001). After $24 \mathrm{~h}$ incubation, chlorophenol red- $\beta$-D- 
galactopyranoside (CPRG, Roche Diagnostics) was dissolved in GM and added to each well to a final concentration of $0.1 \mathrm{mg} / \mathrm{ml}$. Plates were shaken (600 rpm, $2 \mathrm{~min}$.) before further incubation $\left(30^{\circ} \mathrm{C}\right)$. After $24 \mathrm{~h}$ and $48 \mathrm{~h}$ the plates were sealed with a sterile plate seal (Classic SealPlate ${ }^{\circledR}$ Sealing Film, Excel Scientific, Victorville, CA, U.S.A.), shaken (600 rpm, $2 \mathrm{~min}$.), and briefly centrifuged (300 rpm, $10 \mathrm{sec}$., 5810R centrifuge, Eppendorf $A G$ ) before $O D_{540}$ and $\mathrm{OD}_{620}$ measurements (2300 EnSpire ${ }^{\mathrm{TM}}$ Multilabel Reader, Perkin Elmer, Turku, Finland) were taken.

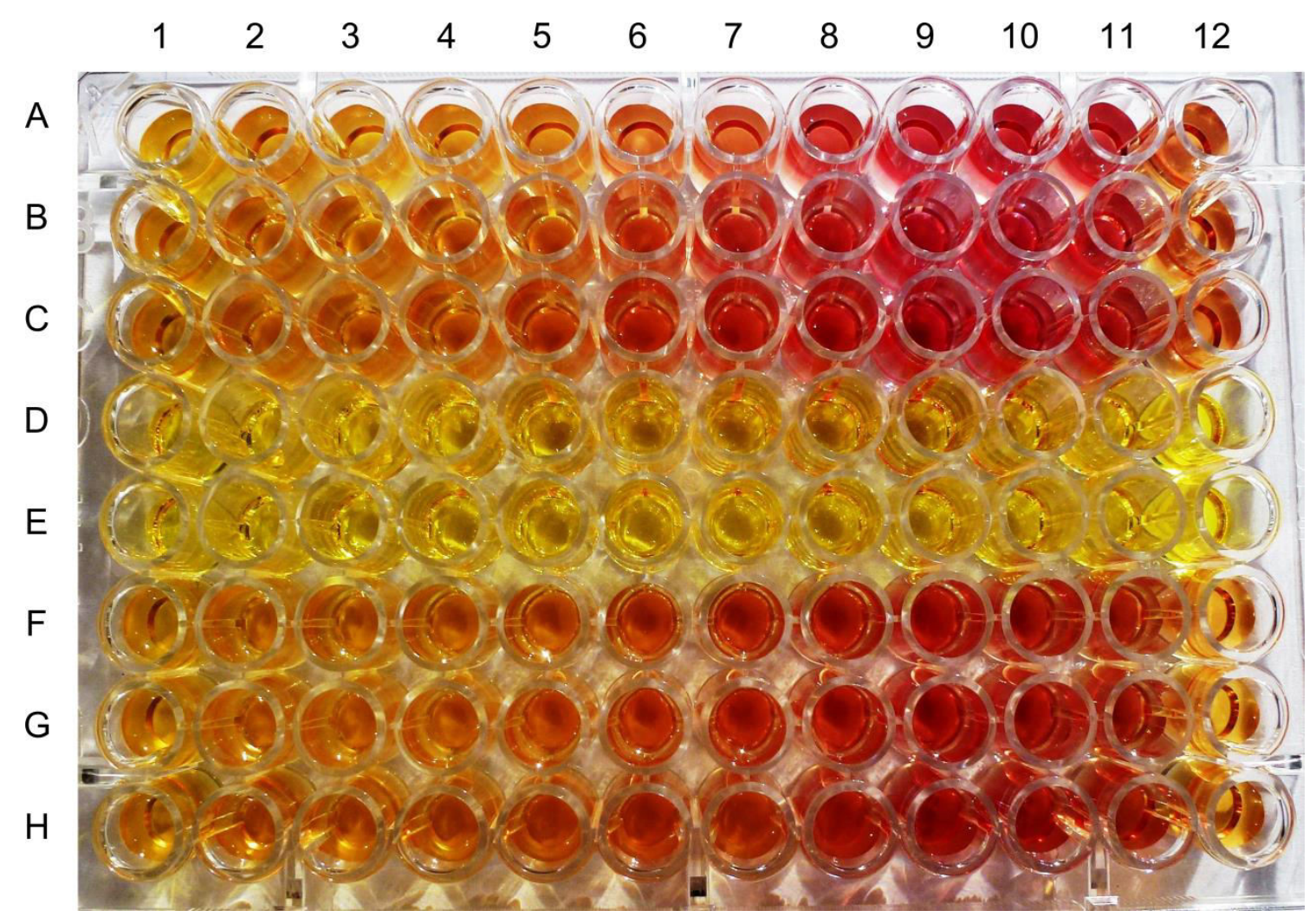

Figure 3.3 Example of a 96-well plate format used in the tunicate yeast bioassays.

Plate showing induction of $\beta$-galactosidase activity in yeast strains exposed to $n$-butylp-aminobenzoate. Yeast strains expressing plasmids pGAL4.BsLBD.VP16 (rows A-C), pGAL4 (rows D-E) and pGAL4.CIBD.VP16 (rows $F-H$ ) were exposed to various concentrations $(1-400 \mu \mathrm{M})$ of $n$-butyl-p-aminobenzoate (columns 3-12). Column 1 contains seeded assay medium (SAM) only and column 2 contains $1 \%$ ethanol solvent plus SAM. 


\subsubsection{Statistics}

To correct for variations in cell densities, the raw $\mathrm{OD}_{540}$ values were normalised using the equation of Routledge and Sumpter (1996):

$\mathrm{OD}_{540}($ corrected $)=$ Sample $\mathrm{OD}_{540}-\left(\right.$ Sample $\mathrm{OD}_{620}-$ Blank $\left.\mathrm{OD}_{620}\right)$.

Blank $O_{620}$ values correspond to wells containing ethanol solvent and SAM, while sample $O D_{540}$ and sample $O D_{620}$ values correspond to wells containing the test chemicals (Section 3.2.6). Sample $\mathrm{OD}_{620}$ values were used to assess general toxicity of test compounds towards yeast cells. The median effective concentrations ( $\mathrm{EC}_{50}$ values) and $95 \%$ confidence intervals $(95 \% \mathrm{Cl})$ were determined from four-parameter sigmoidal concentration-response curves using GraphPad Prism Version 6.03 (GraphPad Software, La Jolla, CA, U.S.A., http://graphpad.com) following Routledge \& Sumpter (1996) and Karimullina et al. (2012).

Intra-plate variability was quantified by calculating the coefficient of variance $(\mathrm{CV}, \%)$. For each compound concentration the mean $\left(\mathrm{x}^{-}.\right)$and the standard deviation (SD) for the three replicate measurements were determined. The CV for each compound concentration was calculated as follows:

$$
\mathrm{CV}=\frac{\mathrm{SD}}{\mathrm{x}_{-}^{-}} \times 100 \%
$$

The average of the individual CVs is reported as the intra-assay CV for each yeast strain (Yang et al. 2014).

To assess plate-to-plate consistency, inter-plate CVs were calculated using a known $C$. intestinalis VDR/PXRa LBD agonist, $n$-butyl-p-aminobenzoate (Ekins et al. 2008). Assays were run in triplicates on three different plates. The plate means for each $n$-butyl-p-aminobenzoate concentration were calculated and then used to calculate the overall mean, SD and CV. The overall CV for each concentration was calculated as follows: 


$$
\mathrm{CV}=\frac{\text { SD of plate } \mathrm{X}_{-}^{-}}{\mathrm{x}^{-} \text {. of plate } \mathrm{X}_{,}^{-}} \times 100 \%
$$

The average of the overall CVs for each compound concentration is reported as the inter-assay CV (Yang et al. 2014).

\subsection{Results and discussion}

\subsubsection{Development of the bioassay yeast strains}

The objective of this chapter was to develop recombinant yeast bioassays in which $\beta$-galactosidase enzymatic activity (encoded by lacZ) was induced by ligands that activate tunicate xenobiotic receptors. There are two main components to the yeast bioassays: the yeast host strain and the plasmid constructs transformed into the yeast.

The yeast ( $S$. cerevisiae) host strain selected for this work was PJ69-4A (MATa,

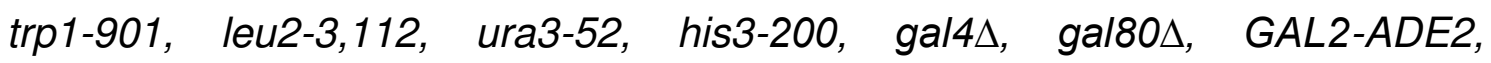
LYS2::GAL1-HIS3, met2::GAL7-lacZ; James et al. 1996). This strain is characterised by multiple genotypic features making it a suitable host strain: (i) trp 1-901: complementation of trp1-901 by the TRP1 gene encoded by pGBKT7derived plasmids allows selection for tryptophan prototrophic transformants on

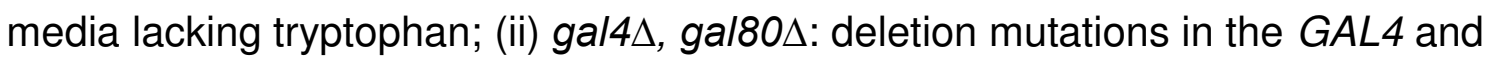
GAL80 nuclear genes render the endogenous GAL4/GAL80 galactose-sensitive induction pathway non-functional (Traven et al. 2006) and; (iii) met2::GAL7lacZ: this modification places a lacZ gene under the control of a GAL4-regulated promoter. The lac $Z$ reporter gene product, E. coli $\beta$-galactosidase enzyme, catalyses hydrolysis of the colorimetric substrate CPRG without the need for cell lysis, thus allowing repeated, non-lethal measurements of the yeast bioassay's output.

All seven plasmids generated in this study encode chimeric proteins combining one or more of three basic elements: (i) a GAL4-DBD; (ii) a tunicate VDR/PXRa 
orthologue LBD and; (iii) a VP16-AD, derived from herpes simplex virus protein 16 (Louvion et al. 1993). While the sequences encoding the GAL4-DBD and VP16-AD are well established from previous studies (Louvion et al. 1993), determination of the appropriate tunicate VDR/PXRa orthologue LBD sequences, to be expressed in this study, required careful consideration. Computer prediction programmes (e.g. Simple Modular Architecture Research Tool (SMART); Schultz et al. 1998; Lubec et al. 2005; Letunic et al. 2012) appeared to underestimate the size of xenobiotic receptor LBDs (Chapter Two, Section 2.4.1). To address this problem, the tunicate VDR/PXRa LBD domain was defined as comprising residues just C-terminal of the predicted DBD through to the $\mathrm{C}$-terminal end of the predicted open reading frame. This is the same region used previously for expression of the $C$. intestinalis VDR/PXR LBD domain in mammalian cell lines (Ekins et al. 2008; Fidler et al. 2012).

The seven plasmids used in this study were as follows. Plasmid pGAL4 is simply the base vector pGBKT7 encoding the GAL4-DBD domain and a short, non-functional C-terminal sequence (Figure 3.2, Appendix 13). Plasmid pGAL4.VP16 encodes the GAL4-DBD fused to the VP16-AD which has been found useful for coupling ligand-activated NRs in yeast to activation of reporter genes (Louvion et al. 1993; Figure 3.2, Appendix 12 and 13). Together, pGAL4 and pGAL4.VP16 are the negative control plasmids that control for reporter gene expression changes in the absence of any tunicate VDR/PXRa LBD domain sequences. Plasmid PGAL4.CIBD expresses a fusion protein consisting of the GAL4-DBD fused to the $C$. intestinalis VDR/PXRa LBD (residues 221-532 of NP 001071847; Figure 3.2, Appendix 12 and 13). Plasmid pGAL4.CLBD.VP16 encodes the same region of the $C$. intestinalis VDR/PXRa LBD as pGAL4.CLBD but, in addition, encodes the VP16-AD on the C-terminus of the expressed protein (Figure 3.2, Appendix 12 and 13). Plasmid pGAL4.CLBD 31 .VP16 is similar to pGAL4.CLBD.VP16 but the LBD is 31 residues shorter with the $\mathrm{C}$-terminal flank of the LBD being that predicted by SMART (i.e. residues 221-501 of NP 001071847; Letunic et al. 2012; Figure 3.2, Appendix 12 and 13). Plasmid pGAL4.BsLBD expresses the B. schlosseri VDR/PXRa LBD residues 39-343 of AHB39790 (Figure 3.2, Appendix 12 and 13). Plasmid PGAL4.BSLBD.VP16 encodes the same region of the $B$. schlosseri 
VDR/PXRa LBD as pGAL4.BsLBD but, in addition, has the VP16-AD on the Cterminus of the expressed fusion protein (Figure 3.2, Appendix 12 and 13).

In total, five plasmids were generated that encoded chimeric proteins containing both the GAL4-DBD and a tunicate VDR/PXRa LBD (Figure 3.2, Appendix 13). Two of these plasmids, pGAL4.CIBD and pGAL4.BSLBD, expressed fusion proteins lacking the VP16-AD (Figure 3.2, Appendix 13). For these chimeric proteins to function, their native ligand-dependent activation domain (AF-2) needs to function within the nuclear milieu of yeast cells. As previous studies have shown that the AF-2 domain of at least some vertebrate NRs functions in yeast (Pham et al. 1992; vom Baur et al. 1998; Gaudon et al. 1999), the two constructs were generated to test if this was also observed in the tunicate VDR/PXRa receptors.

\subsubsection{Yeast bioassay media optimisation}

The permissiveness of vertebrate PXR LBDs with respect to cognate ligand structures (Ekins et al. 2008; Fidler et al. 2012) raised concerns that some yeast growth media components might act as tunicate VDR/PXRa LBD receptor ligands, thereby compromising the yeast bioassay. More specifically, two amino acids (L-tyrosine and L-phenylalanine) present in the original yeast media of Routledge and Sumpter (1996) and Beresford et al. (2000) had aromatic structures suggesting that they might be potential tunicate VDR/PXRa LBD ligands. While preliminary experiments established that neither of these amino acids induced $\beta$-galactosidase enzymatic activity from any of the seven strains tested (data not shown), both amino acids were omitted from the yeast growth media as a precaution.

In the course of preliminary experiments on media composition, it was noted that strains carrying three plasmids (pGAL4.CILBD.VP16, pGAL4.CLBD $\triangle 31 . V P 16$ and pGAL4.BsLBD.VP16) grew faster in the absence of $L$-histidine than strains carrying any of the other four plasmids. To investigate this observation, all seven yeast strains were grown in media lacking L-histidine or supplemented with L-histidine at one of three concentrations, and cell density 
$\left(\mathrm{OD}_{620}\right)$ was measured over time (0-63 h; Figure 3.4). In the absence of Lhistidine in the media, the three strains carrying plasmids that encode a tunicate VDR/PXRa LBD combined with the VP16-AD grew much faster than the other four strains (Figure 3.4A). With increasing concentrations of exogenously supplied L-histidine, the degree of differential growth between the seven strains diminished (Figure 3.4B-D). In media containing both 200 and $322 \mu \mathrm{M}$ Lhistidine there was no observed difference in $\mathrm{OD}_{620}$ values between any of the seven strains at $63 \mathrm{~h}$ of incubation (Figure 3.4C-D). The observed histidinedependent differential growth of the yeast strains can be explained by noting that the yeast host strain genotype (MATa, trp1-901, leu2-3,112, ura3-52, his3200, gal4A, gal80A, GAL2-ADE2, LYS2::GAL1-HIS3, met2::GAL7-lacZ) contains both the his3-200 mutant allele and a functional HIS3 gene under the control of the GAL4-regulated GAL1 promoter. The three chimeric proteins encoded by the plasmids pGAL4.CLBD.VP16, pGAL4.CLBDA31.VP16 and pGAL4.BSLBD.VP16 appeared to enhance transcription of the HIS3 gene from its GAL4-activated GAL1 promoter, leading to L-histidine biosynthesis and enhanced strain growth in L-histidine-limited media (Figure 3.4A, B). This induction of the GAL4-activated GAL1 promoter, even in the absence of any experimentally added inducing compound(s), suggests low background levels of constitutive activity by the three chimeric proteins. Alternatively, the three chimeric proteins may be activated by either endogenously synthesised yeast compounds or by yeast growth media components. In contrast, the GAL4regulated GAL1 promoter of $H I S 3$ was inactive in the four yeast strains carrying plasmids pGAL4, pGAL4.VP16, pGAL4.CIBDD and pGAL4.BSLBD leading to little or no growth in L-histidine-limited media (Figure 3.4A). 

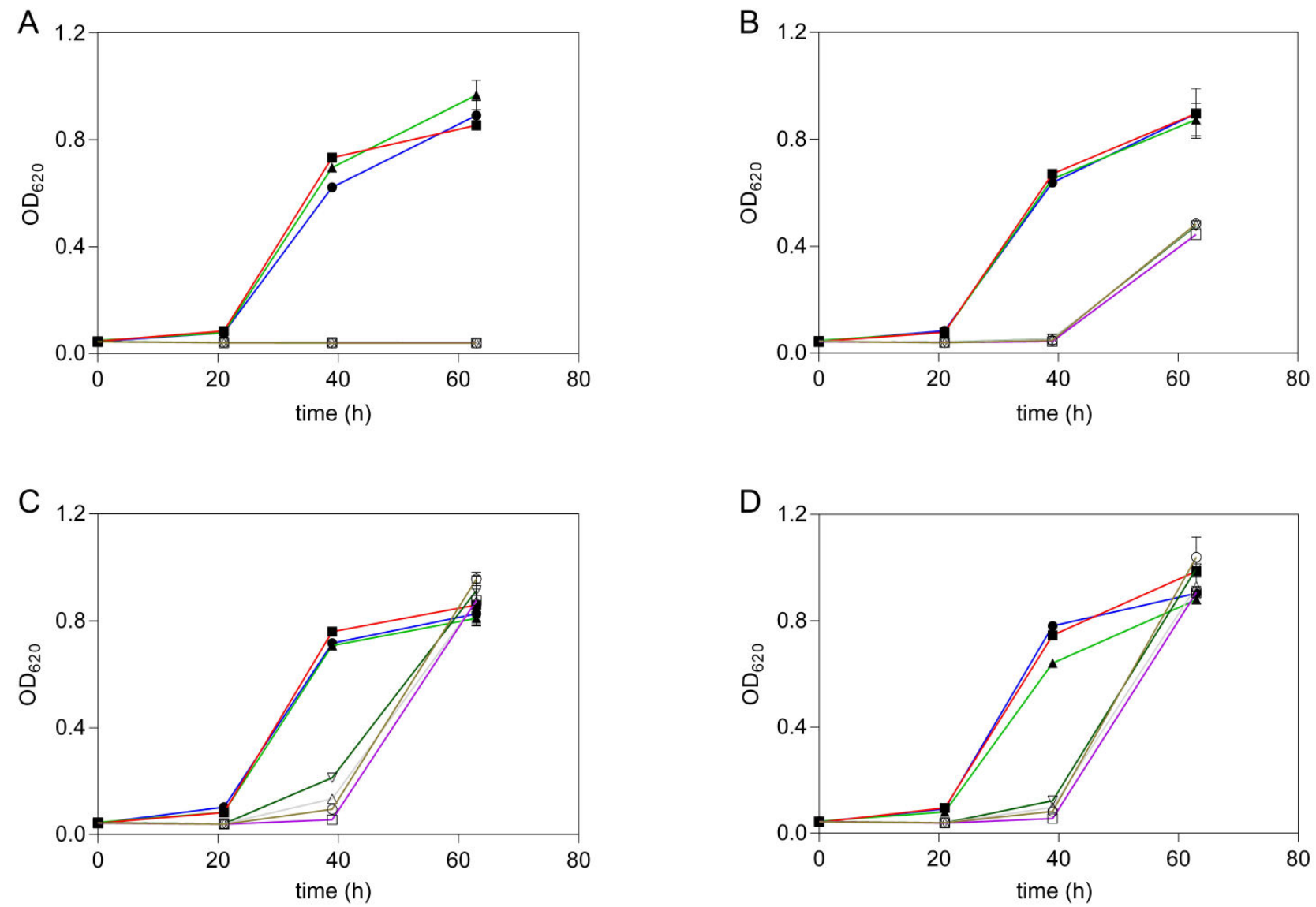

$\bigcirc$ pGAL4

$\square$ pGAL4.VP16

$\triangle$ pGAL4.CILBD

$\rightarrow$ pGAL4.BSLBD

- pGAL4.CiLBD $\triangle 31$.VP16

$\leftarrow$ pGAL4.CiLBD.VP16

- pGAL4.BSLBD.VP16

Figure 3.4 Media concentrations of L-histidine influence the growth rate of three recombinant yeast strains.

Yeast strains carrying the pGBKT7-based expression plasmids described in Section 3.3.1 were grown in liquid growth media with differing concentrations of L-histidine: (A) $0 \mu \mathrm{M}$, (B) $10 \mu \mathrm{M}$, (C) $200 \mu \mathrm{M}$ and (D) $322 \mu \mathrm{M}$. Cell density was quantified by $\mathrm{OD}_{620}$ values and measured at four time points $(0,21,39$ and $63 \mathrm{~h})$ following culture establishment. Data points represent means of $n=3$ replicates. Error bars show \pm one standard deviation. 
To explore this interpretation further, $\beta$-galactosidase induction by an established $C$. intestinalis VDR/PXRa agonist, $n$-butyl- $p$-aminobenzoate (Ekins et al. 2008), was measured in media containing four concentrations of Lhistidine (Figure 3.5). The response curves of the three strains showing $n$-butyl$p$-aminobenzoate-dependent $\beta$-galactosidase induction (i.e. carrying plasmids pGAL4.CLBD.VP16, pGAL4.CLBD $31 . V P 16$ and pGAL4.BSLBD.VP16) became progressively less sigmoidal with increasing concentrations of mediasupplied exogenous L-histidine (Figure 3.5A-D). In addition, the four strains that showed no ligand-dependent induction of $\beta$-galactosidase activity exhibited elevated background levels of $\beta$-galactosidase enzymatic activity at higher Lhistidine concentrations (Figure 3.5A-D). As the E.coli $\beta$-galactosidase protein (http://uniprot.org/uniprot/P00722), encoded by the lac $Z$ reporter gene, contains multiple L-histidine residues it appeared that the $\beta$-galactosidase enzymatic activity induction curves generated in this study are a summation of the liganddependent activation of two GAL4-regulated promoters/genes in the host strain genome, specifically GAL7-lacZ and GAL1-HIS3 (Traven et al. 2006). This suggests that the yeast bioassay developed measures the 'output' of $\beta$ galactosidase enzymatic activity of a single ligand-activated chimeric protein controlling two promoters, both reducing background signal and creating a more sigmoidal induction response (Figure 3.5A-D).

To provide a compromise between the need for exogenous L-histidine, essential for growth of some yeast strains (pGAL4, pGAL4.VP16, pGAL4.CIBD and pGAL4.BSLBD) and the requirement to minimise the background (i.e. not ligand-induced $\beta$-galactosidase enzymatic activity), the seven yeast strains were first grown in the presence of L-histidine to generate the yeast starter stock (Section 3.2.5). The strains carrying plasmids pGAL4, pGAL4.VP16, pGAL4.CLBD and pGAL4.BsLBD were supplied with $322 \mu \mathrm{M}$ L-histidine and strains carrying plasmids pGAL4.CILBD.VP16, pGAL4.CLBD 31 .VP16 and pGAL4.BsLBD.VP16 were supplied with $10 \mu \mathrm{M}$ L-histidine. No exogenous Lhistidine was provided in the SAM (Section 3.2.6) which was used for the yeast bioassay itself. 

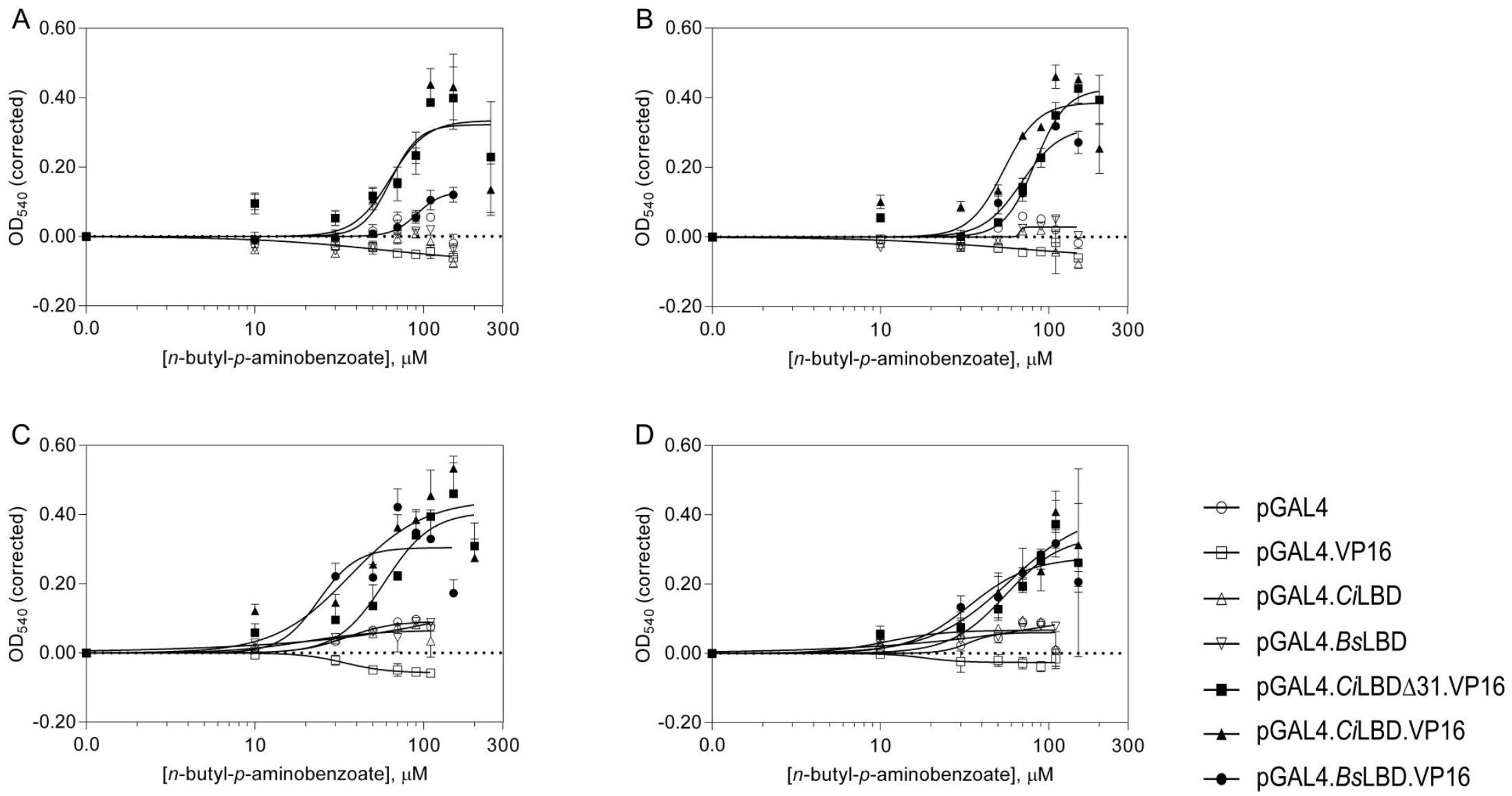

Figure 3.5 Influence of L-histidine concentrations on background activity of $\beta$-galactosidase.

Yeast strains were exposed to $n$-butyl-p-aminobenzoate over a range of concentrations $(0-300 \mu \mathrm{M})$ in growth media containing four concentrations of L-histidine: (A) $0 \mu \mathrm{M}$, (B) $10 \mu \mathrm{M}$, (C) $200 \mu \mathrm{M}$ and (D) $322 \mu \mathrm{M}$. $\beta$-galactosidase enzymatic activities, as measured by

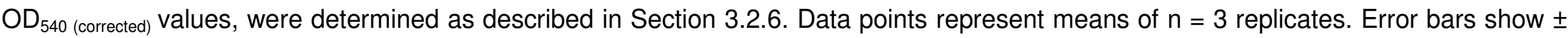
one standard deviation. Dotted lines indicate baseline. 


\subsubsection{Activity of synthetic toxicants in the yeast bioassays}

Having optimised the media conditions for the recombinant yeast strains used in the bioassays, the ability of $n$-butyl-p-aminobenzoate and three additional synthetic chemicals (carbamazepine, bisphenol-A (BPA) and p-aminobenzoic acid) to induce $\beta$-galactosidase enzymatic activity were investigated (Table 3.1, Figure 3.6).

Two of the synthetic chemicals tested (n-butyl-p-aminobenzoate and carbamazepine) were positive controls for the yeast bioassays because they had previously been shown to activate the $C$. intestinalis VDR/PXR $\alpha$ LBD when expressed as a fusion gene in mammalian cell lines (Ekins et al. 2008). $\mathrm{N}$-butyl$p$-aminobenzoate and carbamazepine both induced $\beta$-galactosidase enzymatic activity from the three yeast strains carrying plasmids encoding a tunicate VDR/PXRa LBD fused to the VP16-AD (pGAL4.CIBDA31.VP16, pGAL4.CLBD.VP16 and pGAL4.BsLBD.VP16; Figure 3.6A-B, Table 3.1). Strains carrying the two negative control plasmids, pGAL4 and pGAL4.VP16, showed no evidence of induced $\beta$-galactosidase enzymatic activity, nor did the two strains encoding GAL4.VDR/PXR $\alpha$ LBD fusion proteins lacking the VP16AD (pGAL4.CILBD, pGAL4.BsLBD; Figure 3.6A-B). For some strains there was evidence of reduced $\beta$-galactosidase enzymatic activity at higher concentrations of both $n$-butyl-p-aminobenzoate and carbamazepine (Figure 3.6A, B). This suppression effect does not appear to be an antagonistic effect mediated through the tunicate VDR/PXRa LBD as it is apparent in the negative control strains, being most prominent with plasmid pGAL4.VP16 at high concentrations of carbamazepine (Figure 3.6B). Examination of the $\mathrm{OD}_{620}$ values from the yeast bioassays shows a general toxic effect of $n$-butyl- $p$-aminobenzoate and carbamazepine towards yeast cells, particularly at high concentrations (Appendix 14). The sensitivity of the pGAL4.CLBD.VP16 yeast bioassay ( $n$ butyl-p-aminobenzoate: $\mathrm{EC}_{50}=55 \mu \mathrm{M}$; carbamazepine: $\mathrm{EC}_{50}=6.2 \mu \mathrm{M}$; Table 3.1) was comparable to that of the mammalian cell line bioassay expressing $C$. intestinalis VDR/PXRa (n-butyl-p-aminobenzoate: $\quad \mathrm{EC}_{50}=16.5 \mu \mathrm{M}$; carbamazepine: $\mathrm{EC}_{50}>10 \mu \mathrm{M}$; Ekins et al. 2008). The yeast strain expressing the shorter version of the CNDR/PXRa LBD (pGAL4.CLBD 31 .VP16) was 
slightly less sensitive to both $n$-butyl-p-aminobenzoate and carbamazepine than strains expressing the longer version (pGAL4.CLBD.VP16) as indicated by the shape of the inductive response and $\mathrm{EC}_{50}$ values (Figure 3.6A-B, Table 3.1).

Two additional synthetic chemicals ( $p$-aminobenzoic acid and BPA), with planar structures and at least one hydrogen bond acceptor (Figure 3.1), were tested in the yeast bioassays (Table 3.1, Figure 3.6C-D). Both induced positive responses although these were non-sigmoidal. The B. schlosseri VDR/PXR $\alpha$ LBD-based yeast bioassay (pGAL4.BsLBD.VP16) was less sensitive than the C. intestinalis VDR/PXRa equivalent (Figure 3.6C, D, Table 3.1). Activity of $p$ aminobenzoic acid in the mammalian cell line bioassay expressing CNDR/PXRa had been noted previously although the yeast bioassay appears to be more sensitive ( $p$-aminobenzoic acid: yeast $\mathrm{EC}_{50}=3 \mu \mathrm{M}$; mammalian $\mathrm{EC}_{50}=10-30 \mu \mathrm{M} ; \mathrm{M}$. Krasowski, personal communication). Bisphenol-A, an estrogenic endocrine disruptor (Olea et al. 1996; Welshons et al. 2003), has been identified as a potent toxicant when tested on tunicate larvae (Mansueto et al. 2011; Cangialosi et al. 2013) with low concentrations (3-10 $\mu \mathrm{M})$ of BPA affecting swimming, hatching and normal development of $C$. intestinalis larvae (Matsushima et al. 2013). Given the comparable sensitivities of the pGAL4.CIBDD.VP16-based yeast bioassays $\left(\mathrm{EC}_{50}=9.2 \mu \mathrm{M}\right.$; Table 3.1) these yeast bioassays may, at least in some contexts, provide cheap and consistent proxies for assessing the effects of environmental contaminants on invertebrate members of coastal ecosystems (Stewart et al. 2014). To further establish the validity of this idea, it would be useful to systematically test synthetic chemicals known to be toxic to $C$. intestinalis and $B$. schlosseri in the yeast bioassays (Bellas et al. 2003).

The failure of yeast strains carrying plasmids pGAL4.CILBD and pGAL4.BSLBD to display ligand-dependent induction of $\beta$-galactosidase enzymatic activity indicates that any transcription AF-2 domain within a tunicate VDR/PXRa LBD is non-functional in yeast cells (Berry et al. 1990; Louvion et al. 1993). Future yeast bioassays based around the template described in this thesis should incorporate a C-terminal VP16-AD domain onto any fusion proteins generated. 
Table 3.1 Activation of the tunicate yeast bioassays by four synthetic chemicals.

Ligand-dependent induction of $\beta$-galactosidase enzymatic activity was measured in yeast strains carrying plasmids pGAL4.CILBDA31.VP16, pGAL4.CILBD.VP16 or pGAL4.BSLBD.VP16. Mean effective concentrations (EC 50 values) and 95\% confidence intervals $(95 \% \mathrm{Cl})$ are given in $\mu \mathrm{M}$. 95\% confidence intervals represent variation within a triplicate measurement $(\mathrm{n}$ = 3). Coefficients of variance (CV) are given in \% for triplicate intra-plate measurements. All compounds were dissolved in ethanol.

\begin{tabular}{|c|c|c|c|c|c|c|c|c|}
\hline \multirow[t]{2}{*}{ Chemical name } & \multirow[t]{2}{*}{ Toxin type } & \multirow{2}{*}{$\begin{array}{l}\text { Supplier } \\
\text { (Catalogue No.) }\end{array}$} & \multicolumn{2}{|c|}{ pGAL4.CiLBD $\triangle 31 . V P 16$} & \multicolumn{2}{|c|}{ pGAL4.CiLBD.VP16 } & \multicolumn{2}{|c|}{ pGAL4.BsLBD.VP16 } \\
\hline & & & $\mathrm{EC}_{50}(95 \% \mathrm{Cl})$ & CV & $\mathrm{EC}_{50}(95 \% \mathrm{Cl})$ & CV & $\mathrm{EC}_{50}(95 \% \mathrm{Cl})$ & CV \\
\hline $\begin{array}{l}N \text {-butyl- } p \text {-amino- } \\
\text { benzoate }^{\text {a }}\end{array}$ & $\begin{array}{l}\text { Local } \\
\text { anaesthetic }\end{array}$ & $\begin{array}{l}\text { Sigma-Aldrich } \\
\text { (B7753) }\end{array}$ & $68(64-74)$ & 16 & $55(48-63)$ & 11 & $57(47-69)$ & 14 \\
\hline Carbamazepine $^{\mathrm{b}}$ & Anticonvulsant & $\begin{array}{l}\text { Sigma-Aldrich } \\
(\mathrm{C} 4024)\end{array}$ & $7.1(4.9-9.9)$ & 18 & $6.2(5.3-7.3)$ & 7 & $7.0(4.9-9.8)$ & 21 \\
\hline$P$-aminobenzoic acid ${ }^{\mathrm{b}}$ & Supplement & $\begin{array}{l}\text { Sigma-Aldrich } \\
\text { (A9878) }\end{array}$ & $1.8(1.2-2.9)$ & 14 & $3.0(1.1-8.8)$ & 25 & 191 (DNC) & 89 \\
\hline Bisphenol- $A^{b}$ & EDC & $\begin{array}{l}\text { Sigma-Aldrich } \\
\text { (B1760) }\end{array}$ & $5.3(1.8-15.6)$ & 18 & $9.2(4.9-17.5)$ & 14 & $10(0-4108)$ & 85 \\
\hline
\end{tabular}

Abbreviations: EDC, endocrine disrupting chemical.

${ }^{a}$ Strains were incubated for 24 hours.

${ }^{\mathrm{b}}$ Strains were incubated for 48 hours. 

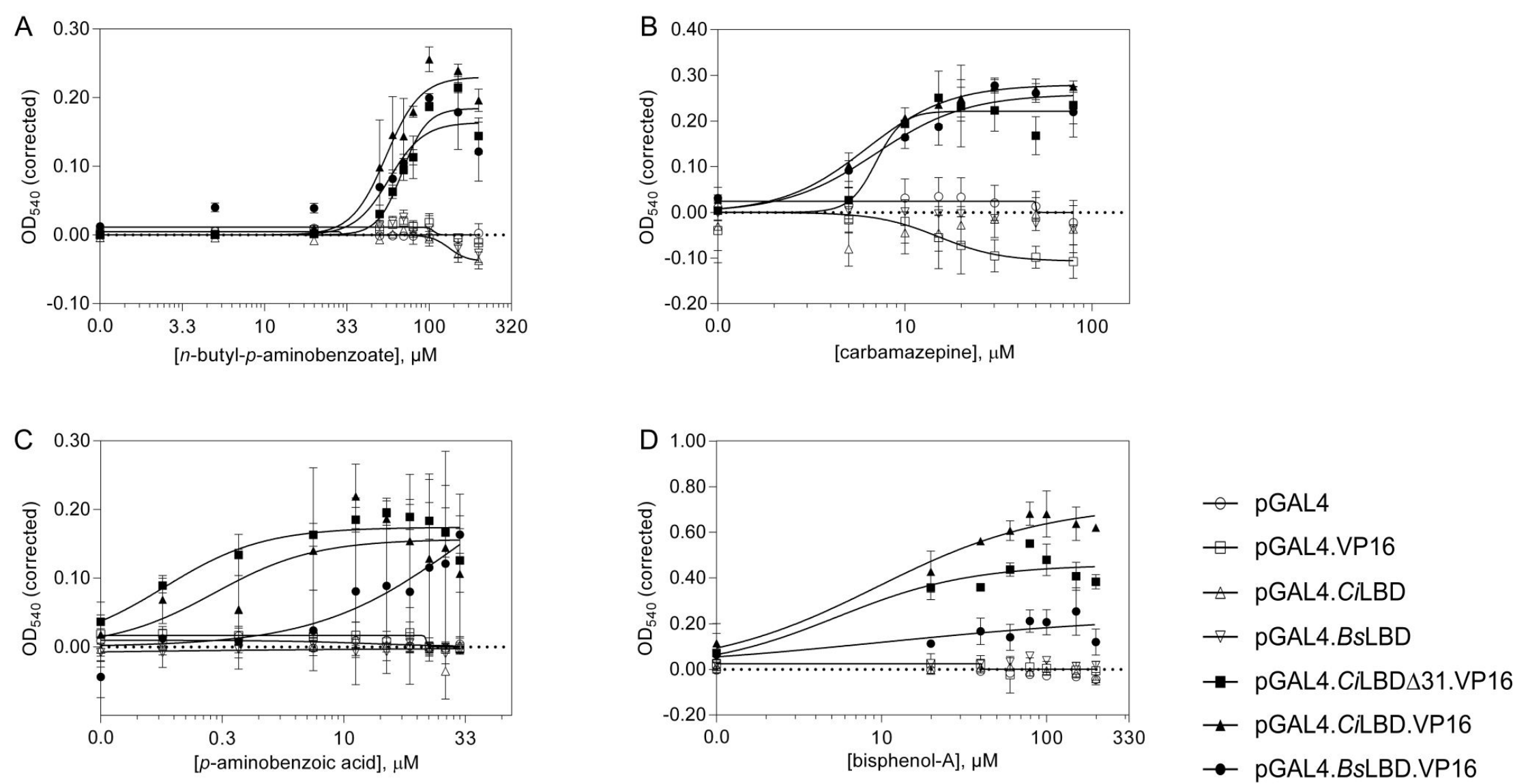

Figure 3.6 Activities of four synthetic chemicals tested in the yeast bioassays.

Yeast strains carrying seven pGBKT7-based expression vectors were exposed to a range of concentrations of: (A) $n$-butyl- $p$ aminobenzoate, (B) carbamazepine, (C) p-aminobenzoic acid and (D) bisphenol-A. $\beta$-galactosidase enzymatic activities, as measured by

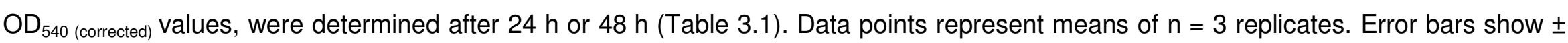
one standard deviation. Dotted lines indicate baseline. 


\subsubsection{Activity of natural microalgal biotoxins in the yeast bioassays}

Pursuing the hypothesis that the ecologically relevant ligands of tunicate VDR/PXRa receptors include those toxins naturally present in a marine filterfeeder's diet, three established microalgal biotoxins (okadaic acid, PTX-11 and portimine; Figure 3.1) were tested in the yeast bioassays. All three microalgal biotoxins induced $\beta$-galactosidase enzymatic activity from those strains carrying plasmids encoding a tunicate VDR/PXRa LBD fused to the VP16-AD: pGAL4.CILBD 31 .VP16, pGAL4.CIBDD.VP16 and pGAL4.BsLBD.VP16 (Table 3.2, Figure $3.7 A-C)$. In contrast, the yeast strains carrying the two negative control plasmids, pGAL4 and pGAL4.VP16, showed no evidence of induced $\beta$ galactosidase enzymatic activity nor did the two strains encoding GAL4.VDR/PXRa LBD fusion proteins lacking a C-terminal VP16-AD domain (Figure $3.7 \mathrm{~A}-\mathrm{C}$ ). There was no indication of toxicity of microalgal biotoxins towards the yeast cells within the concentration ranges tested (Figure 3.7A-C).

Two freshwater cyanobacterial toxins (microcystin-RR and anatoxin-A) were also tested in the yeast bioassays. They were considered to be 'negative controls' as being of freshwater origin, they would not have been encountered by marine tunicates over evolutionary time. As expected both were inactive in the yeast bioassays (Table 3.2).

Identification of portimine as an activator of both tunicate VDR/PXRa LBDs tested (Table 3.2) is consistent with its reported toxicity when tested using mammalian cells in vitro $\left(\mathrm{LC}_{50}=2.7 \mathrm{nM}\right)$ suggesting that portimine may be toxic towards a wide range of chordates - including tunicates (Selwood et al. 2013). The yeast strain expressing the shorter version of the $C$. intestinalis VDR/PXRa LBD (pGAL4.CLBD $\triangle 31$.VP16) was more strongly activated by portimine than the strain expressing the longer version (pGAL4.CLBD.VP16) as indicated by the plateau levels of $\beta$-galactosidase production (Figure 3.7C). This difference was not reflected in $\mathrm{EC}_{50}$ values (Table 3.2). In contrast, the yeast strain expressing the shorter version of the $C$. intestinalis VDR/PXRa LBD was less strongly activated by okadaic acid and PTX-11 as reflected in plateau levels of $\beta$-galactosidase activity (Figure 3.7A-B). The $E_{50}$ values for the pGAL4.CLBD $\triangle 31 . V P 16$ strain were lower than for the pGAL4.CIBDD.VP16 
strain for both okadaic acid and PTX-11 (Table 3.2). The $\mathrm{EC}_{50}$ values for activation of the full-length $C$. intestinalis VDR/PXRa LBD by okadaic acid (pGAL4.CILBD.VP16 $\mathrm{EC}_{50}=27 \mathrm{nM}$; Table 3.2) is comparable with the value reported using mammalian cell line CNDR/PXRa-based bioassays $\left(E C_{50}=18.2 \mathrm{nM}\right)$ supporting the validity of the yeast bioassays (Fidler et al. 2012).

Two of the microalgal biotoxins that activated the tunicate VDR/PXRa LBDs in this study (okadaic acid and PTX-11) are produced by dinoflagellate species within the cosmopolitan genus Dinophysis, which can reach cell densities of $10^{2}-10^{5}$ cells $/ L$ in coastal marine waters (Reguera et al. 2012). Thus, it is to be expected that filter-feeding marine invertebrates will encounter and accumulate such biotoxins through their diet (Sekiguchi et al. 2001; Echevarria et al. 2012; Roje-Busatto \& Ujević 2014). Although the toxicity of microalgal biotoxins towards tunicates, both adult and tadpole, requires more investigation, the yeast bioassay data presented in this chapter is consistent with the speculation that tunicate VDR/PXRa LBDs have adaptively evolved to bind commonly encountered marine biotoxins (Fidler et al. 2012). Further investigations are required to assess the toxicity of structurally diverse microalgal biotoxins towards tunicates. These studies would be complemented by determination of microalgal biotoxin $\mathrm{EC}_{50}$ values using the yeast bioassays described here. 
Table 3.2 Activation of the tunicate yeast bioassays by five algal toxins.

Ligand-dependent induction of $\beta$-galactosidase enzymatic activity was measured in yeast strains carrying plasmids pGAL4.CILBDA31.VP16, pGAL4.CILBD.VP16 or pGAL4.BSLBD.VP16. Mean effective concentrations (EC 50 values) and 95\% confidence intervals $(95 \% \mathrm{Cl})$ are given in $\mathrm{nM}$. 95\% confidence intervals represent variation within a triplicate measurement $(\mathrm{n}$ = 3). Coefficients of variance (CV) are given in \% for triplicate intra-plate measurements. All compounds were dissolved in ethanol.

\begin{tabular}{|c|c|c|c|c|c|c|c|c|}
\hline \multirow[t]{2}{*}{ Chemical name } & \multirow[t]{2}{*}{ Toxin type } & \multirow{2}{*}{$\begin{array}{l}\text { Supplier } \\
\text { (Catalogue No.) }\end{array}$} & \multicolumn{2}{|c|}{ pGAL4.CILBD $\triangle 31 . V P 16$} & \multicolumn{2}{|c|}{ pGAL4.CiLBD.VP16 } & \multicolumn{2}{|c|}{ pGAL4.BsLBD.VP16 } \\
\hline & & & $\mathrm{EC}_{50}(95 \% \mathrm{Cl})$ & CV & $\mathrm{EC}_{50}(95 \% \mathrm{Cl})$ & CV & $\mathrm{EC}_{50}(95 \% \mathrm{Cl})$ & CV \\
\hline 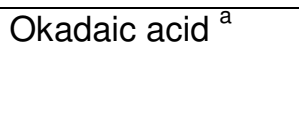 & $\begin{array}{l}\text { Microalgal } \\
\text { biotoxin }\end{array}$ & $\begin{array}{l}\text { Sapphire Bioscience } \\
\text { (AB120375) }\end{array}$ & $19(1.1-327)$ & 46 & $27(15.4-46.4)$ & 8 & $16(8.3-30.4)$ & 8 \\
\hline Pectenotoxin- $11^{a}$ & $\begin{array}{l}\text { Microalgal } \\
\text { biotoxin }\end{array}$ & $\begin{array}{l}\text { MacKenzie et al. } \\
2013\end{array}$ & 633 (117-3437) & 66 & $883(134-5834)$ & 35 & $553(3.4-90,840)$ & 46 \\
\hline Portimine $^{a}$ & $\begin{array}{l}\text { Microalgal } \\
\text { biotoxin }\end{array}$ & Selwood et al. 2013 & $143(114-180)$ & 10 & $130(105-162)$ & 12 & $124(77-202)$ & 7 \\
\hline Microcystin-RR ${ }^{a}$ & $\begin{array}{l}\text { Cyanobacterial } \\
\text { toxin }\end{array}$ & $\begin{array}{l}\text { DHI Lab Products } \\
\text { (PPS-MCRR) }\end{array}$ & $\mathrm{NI}(\mathrm{DNC})$ & DNC & $\mathrm{NI}(\mathrm{DNC})$ & DNC & $\mathrm{NI}(\mathrm{DNC})$ & DNC \\
\hline Anatoxin- $A^{a}$ & $\begin{array}{l}\text { Cyanobacterial } \\
\text { toxin }\end{array}$ & $\begin{array}{l}\text { NRC Canada } \\
\text { (IMB-CRM-ATX) }\end{array}$ & $\mathrm{NI}(\mathrm{DNC})$ & DNC & $\mathrm{NI}(\mathrm{DNC})$ & DNC & $\mathrm{NI}(\mathrm{DNC})$ & DNC \\
\hline
\end{tabular}

Abbreviations: DNC, did not compute; $\mathrm{NI}$, no induction of $\beta$-galactosidase enzymatic activity.

${ }^{a}$ Strains were incubated for 48 hours. 

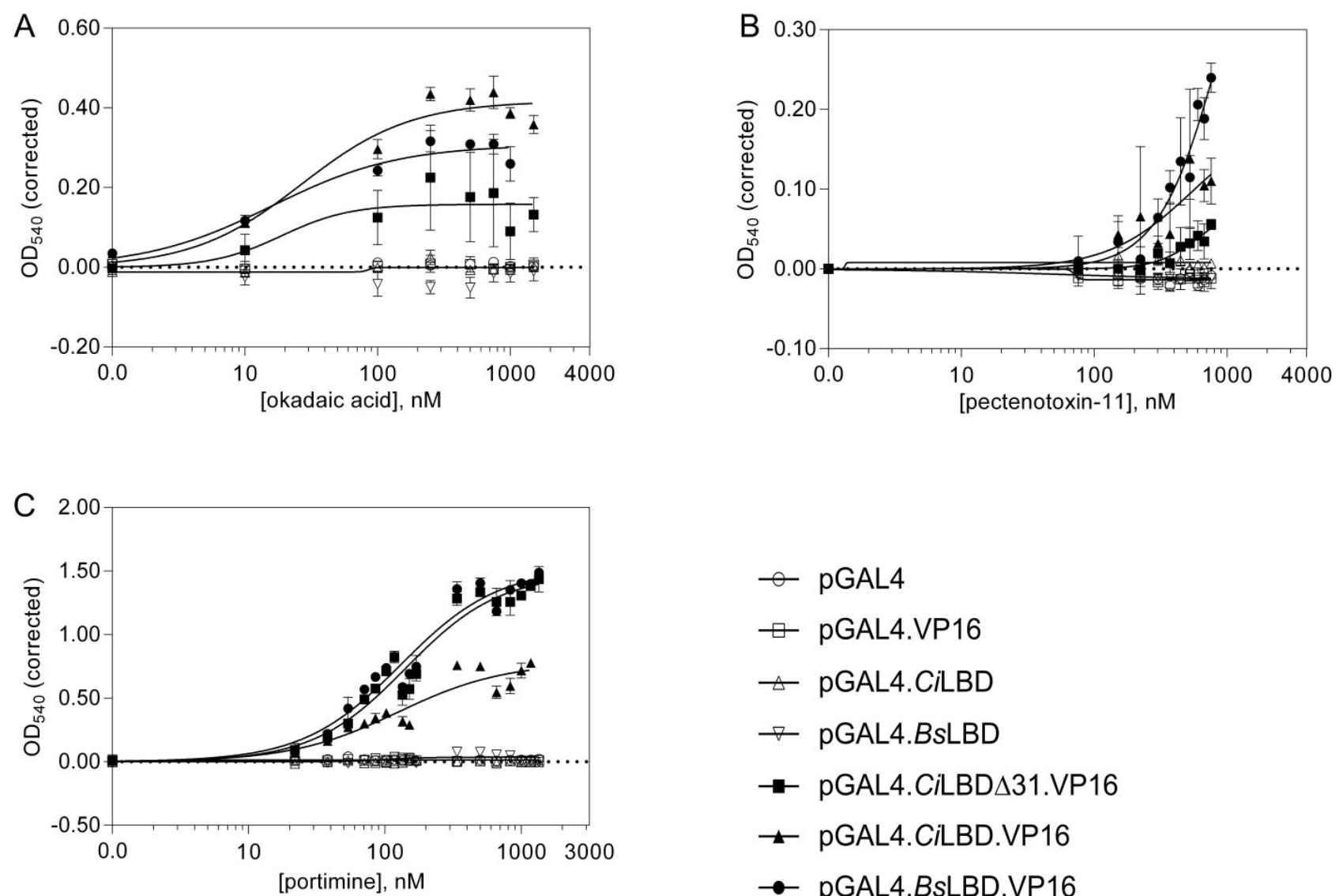

Figure 3.7 Activities of three microalgal biotoxins tested in the yeast bioassays.

Yeast strains carrying seven pGBKT7-based expression vectors were exposed to a range of concentrations of (A) okadaic acid, (B) pectenotoxin-11 and (C) portimine. $\beta$-galactosidase enzymatic activities, as measured by $\mathrm{OD}_{540 \text { (corrected) values, were determined after }}$ $48 \mathrm{~h}$. Data points represent means of $n=3$ replicates. Error bars show \pm one standard deviation. Dotted lines indicate baseline. 


\subsubsection{Characteristics of the yeast bioassays}

Precision of the yeast bioassay was determined using the repeatability between three replicate assays of $n$-butyl- $p$-aminobenzoate reported as inter-plate CVs (Section 3.2.7). Coefficients of variance ranged from $4 \%$ in positive yeast strains to $30 \%$ in negative strains (Table 3.3). The low CV reported for three yeast strains (pGAL4.CLBDA31.VP16, pGAL4.CILBD.VP16 and pGAL4.BSLBD.VP16; CV $<5 \%$ ) indicates that the yeast bioassay test results are consistent and repeatable. The elevated CV values (11-30\%) observed in the four negative yeast strains (pGAL4, pGAL4.VP16, pGAL4.CLBD and pGAL4.BSLBD) can be explained by the lack of induction of $\beta$-galactosidase activity in these strains. This causes negative values for some ligand concentrations making the CV calculation difficult, since CVs can only be computed from non-negative values.

Table 3.3 Inter-plate variability of the tunicate yeast bioassays.

Yeast strains carrying the seven pGBKT7-based expression plasmids were exposed to varying concentrations of $n$-butyl-p-aminobenzoate $(0-200 \mu \mathrm{M})$ in three separate bioassays. Triplicate measurements were taken within each bioassay as described in Section 3.2.7. Results are presented as grand mean, standard deviation (SD) and coefficient of variance (CV, \%).

\begin{tabular}{lll}
\hline Strains & Mean $(\mathrm{SD})$ & CV \\
\hline pGAL4 & $0.093( \pm 0.023)$ & 21 \\
pGAL4.VP16 & $0.040( \pm 0.012)$ & 30 \\
pGAL4.CLBD & $0.140( \pm 0.015)$ & 11 \\
pGAL4.BSLBD & $0.086( \pm 0.010)$ & 12 \\
pGAL4.CLBD $31 . V P 16$ & $0.268( \pm 0.013)$ & 5 \\
pGAL4.CLBD.VP16 & $0.302( \pm 0.013)$ & 4 \\
pGAL4.BSLBD.VP16 & $0.294( \pm 0.016)$ & 5 \\
\hline
\end{tabular}


Due to the size of this study, it was necessary to run samples on multiple assay plates. Intra-assay CVs were calculated to validate these yeast bioassay results for each test compound. Coefficients of variance were $<25 \%$ for two synthetic chemicals, $n$-butyl-p-aminobenzoate and carbamazepine, used as positive controls for the yeast bioassays (Table 3.1). Reliable results for two additional synthetic chemicals, $p$-aminobenzoic acid and BPA, were obtained in yeast strains expressing the $C$. intestinalis VDR/PXRa LBD (pGAL4.CLBD 431 .VP16 and PGAL4.CLBD.VP16; CV <25\%). In contrast, induction of $\beta$-galactosidase by these compounds was highly variable $(\mathrm{CV}>85 \%)$ in yeast strains expressing the B. schlosseri VDR/PXRa LBD (Table 3.1). The yeast bioassays were highly sensitive towards two microalgal biotoxins, portimine and okadaic acid, as indicated by low CV values (CV <25\%; Table 3.2). One exception was the yeast strain expressing the truncated version of the $C$. intestinalis VDR/PXRa LBD with $\beta$-galactosidase activities being highly variable in response to okadaic acid ( $C V=46 \%$; Table 3.2 ). Induction of $\beta$-galactosidase activity in response to PTX11 was very inconsistent in all three positive yeast strains (CV $=35-46 \%$; Table 3.2).

Based on the assay variability observed for BPA, p-aminobenzoic acid and PTX-11, it appears that the yeast bioassay is not sensitive enough to reliably detect these compounds. This may be due to the $\beta$-galactosidase concentration responses being very low for BPA, $p$-aminobenzoic acid and PTX-11 (corrected $\mathrm{OD}_{540}<0.2$; Figure 3.6C-D and 3.7B). In comparison, induction of $\beta$ galactosidase activity by portimine was at least three-times greater than for PTX-11 (corrected $\mathrm{OD}_{540}=0.6-1.5$; Figure $3.7 \mathrm{~B}, \mathrm{C}$ ) resulting in very low intraassay variability (CV $=7-12 \%$; Table 3.2$)$.

The observed variability may also be attributed to low or limited solubility of some test compounds in ethanol. Thus, all compounds were dissolved in DMSO (Section 3.2.1). Of the nine compounds tested, three ( $n$-butyl-p-aminobenzoate, BPA and PTX-11) were active in the yeast bioassays (Appendix 11). Although the yeast bioassays were more sensitive towards $n$-butyl-p-aminobenzoate when dissolved in DMSO than in ethanol $\left(\mathrm{EC}_{50}=2.4-9.4 \mu \mathrm{M}\right.$; Table 3.1, Appendix $11 \mathrm{~A}$ ), the within-assay variability was greater (CV $>25 \%$; Table 3.1 , Appendix 11A). The opposite effect was observed for BPA. When dissolved in 
DMSO, assay sensitivity decreased by one order of magnitude $\left(E_{50}=58-76\right.$ $\mu \mathrm{M}$; Appendix 11A), while intra-assay variability improved (CV $<17 \%$; Appendix $11 \mathrm{~A})$. It appeared that both solvents have differential effects on assay sensitivity for particular compounds within the concentration ranges tested in this study. This is consistent with a previous study showing that the yeast estrogen receptor $(E R \alpha)$ reporter assay is more sensitive using ethanol as a solvent compared to DMSO (Bovee et al. 2004).

The yeast bioassays showed similar levels of low sensitivity and high variability $\left(E_{50}=545-913 \mathrm{nM}, \mathrm{CV}=35-66 \%\right.$; Table 3.2; Appendix 11B $)$ to PTX-11 dissolved in either ethanol or DMSO. These $\mathrm{EC}_{50}$ values are one order of magnitude higher than the $\mathrm{EC}_{50}$ value reported for pectenotoxin-2 (PTX-2; $\mathrm{EC}_{50}$ $=37 \mathrm{nM}$ ) in a mammalian cell line (Fidler et al. 2012). It is important to note that two different pectenotoxin analogues were used in the two studies. Pectenotoxin-11, used in this study, differs slightly from PTX-2 in having an additional hydroxyl group at C 34 (Suzuki et al. 2006). This may change its affinity for the receptor LBD slightly. Both PTX analogues are well known microalgal biotoxins, sharing the same mode of action which can cause diarrhetic shellfish poisoning in vertebrates (Dominguez et al. 2010; Reguera et al. 2012), thus being likely ligands of tunicate VDR/PXRs.

A range of additional observations arise from this study that should be considered in developing similar/alternative yeast bioassays based on VDR/PXR orthologues from tunicate species. Yeast strains producing chimeric proteins that have the GAL4-DBD and VDR/PXRa LBD but lacking the VP16$A D$ showed no ligand-dependent induction of $\beta$-galactosidase enzymatic activity. This indicates that the transcription activation domains expected to be present within VDR/PXRa LBDs are not functional in yeast cells (Berry et al. 1990; Louvion et al. 1993). Therefore, any similar/alternative yeast bioassays developed in future should include a C-terminal VP16-AD in the chimeric proteins designed. The differing responses of yeast strains expressing $C$. intestinalis VDR/PXRa LBDs of varying length highlights a need to consider the effect of the expressed LBD region on the yeast bioassay responses to test compounds. In general, it appeared that for VDR/PXR proteins it is important to 
include all sequences $\mathrm{C}$-terminal to the predicted $\mathrm{DBD}$ rather than relying upon computer programs to delimit the $\mathrm{N}$ - and $\mathrm{C}$-terminal boundaries of the LBD.

\subsection{Conclusion}

Recombinant yeast strains were generated that express two orthologue tunicate VDR/PXRa LBDs fused, at their N-termini, with the generic GAL4-DBD and, at their C-termini, with a generic transcription activation domain (VP16-AD). These chimeric proteins were expressed in a yeast host strain, which encodes a lacZ reporter gene adjacent a GAL4-controlled promoter. The resulting strains increase $\beta$-galactosidase enzymatic activity in a concentration-dependent manner in the presence of putative VDR/PXR LBD ligands. This effect was not found in strains transformed with plasmids encoding proteins with the GAL4DBD and VP16-AD but lacking a VDR/PXRa LBD, proving that the $\beta$ galactosidase induction effect was mediated through the tunicate VDR/PXR $\alpha$ LBDs.

These yeast bioassays proved useful for the detection of both synthetic chemicals and natural microalgal biotoxins. The yeast bioassay was highly reliable for repeated detection of a well-known $C$. intestinalis VDR/PXRa LBD agonist, $n$-butyl-p-aminobenzoate (Ekins et al. 2008). However, assay sensitivity varied depending on the type of compounds tested and the organic solvent used. These data indicated that both induction of $\beta$-galactosidase enzymatic activity (measured as corrected $O D_{540}$ values) greater than 0.2 and sigmoidal response curves are important for achieving trustworthy results (CVs $<25 \%$ ). Future research should therefore aim to focus on these criteria in order to improve assay sensitivity and reliability. This may be achieved by first determining the ideal solvent for a particular compound before being tested in the yeast bioassay (Bovee et al. 2004). The percentage of the solvent used in the final bioassay may also influence sensitivity (Bovee et al. 2004). It may, in some cases, be better to use DMSO because this solvent does not evaporate as quickly as ethanol, which may minimise assay variability. 
The $\beta$-galactosidase enzymatic activity induction curves that were observed were found to be dependent on the histidine concentration in the yeast bioassay media. This indicates that induction of $\beta$-galactosidase activity is the actual sum of activation of two GAL4-DBD-activated co-regulated promoters. While this reflects the specific yeast host strain selected, it appears to result in clearer ligand-dependent response curves suggesting that the specific host strain used in this work has inherent advantages for GAL4-DBD-based yeast bioassays.

Despite the success of the yeast bioassays for some test chemicals described in this chapter, it is important to be aware of a limitation common to all cellbased assays. The test chemicals must cross the cell membrane, either passively or by active transport. They also must be available within the cell cytoplasm in a form that can interact with the chimeric fusion protein (Norcliffe et al. 2013). In addition, yeast cells possess specific mechanisms for the elimination of exogenous toxic compounds, which may reduce the sensitivity of the yeast bioassays to such chemicals (Norcliffe et al. 2013). While such considerations do not undermine the usefulness and validity of these yeast bioassays, they may limit the range of chemicals for which bioactivity can be detected.

\subsection{References}

Banerjee P., Kintzios S., Prabhakarpandian B. 2013. Biotoxin detection using cell-based sensors. Toxins 5(12): 2366-83. doi: 10.3390/toxins5122366

Bellas J., Beiras R., Vazquez E. 2003. A standardisation of Ciona intestinalis (Chordata, Ascidiacea) embryo-larval bioassay for ecotoxicological studies. Water Research 37(19): 4613-22. doi: 10.1016/s00431354(03)00396-8

Beresford N., Routledge E.J., Harris C.A., Sumpter J.P. 2000. Issues arising when interpreting results from an in vitro assay for estrogenic activity. Toxicology and Applied Pharmacology 162(1): 22-33. doi: 10.1006/taap.1999.8817

Berry M., Metzger D., Chambon P. 1990. Role of the two activating domains of the estrogen receptor in the cell-type and promoter-context dependent agonistic activity of the antiestrogen 4-hydroxytamoxifen. EMBO Journal 9(9): 2811-8.

Botana L.M., Alfonso A., Botana A., Vieytes M.R., Vale C., Vilarino N., Louzao C. 2009. Functional assays for marine toxins as an alternative, high- 
throughput-screening solution to animal tests. Trends in Analytical Chemistry 28(5): 603-11. doi: 10.1016/j.trac.2009.02.014

Bovee T.F.H., Helsdingen R.J.R., Koks P.D., Kuiper H.A., Hoogenboom R.L.A.P., Keijer J. 2004. Development of a rapid yeast estrogen bioassay, based on the expression of green fluorescent protein. Gene 325(0): 187-200. doi: 10.1016/j.gene.2003.10.015

Bovee T.F.H., Hendriksen P.J.M., Portier L., Wang S., Elliott C.T., van Egmond H.P., Nielen M.W.F., Peijnenburg A., Hoogenboom L.A.P. 2011. Tailored microarray platform for the detection of marine toxins. Environmental Science and Technology 45(20): 8965-73. doi: 10.1021/es2011385

Buckland G. 2010. Implementing scientifically-robust and humane shellfish toxicity testing: we're still waiting. Alternatives to Laboratory Animals 38(5): 419-26.

Cangialosi M.V., Mansueto V., Faqi A.S. 2013. Bisphenol A (BPA) and atrazine inhibit the embryonic development of Ciona intestinalis (Ascidiacea, Urochordata). Caryologia 66(2): 97-102. doi: 10.1080/00087114.2013.780438

De Boever P., Demare W., Vanderperren E., Cooreman K., Bossier P., Verstraete W. 2001. Optimization of a yeast estrogen screen and its applicability to study the release of estrogenic isoflavones from a soygerm powder. Environmental Health Perspectives 109(7): 691-7. doi: 10.1289/ehp.01109691

Dominguez H.J., Paz B., Daranas A.H., Norte M., Franco J.M., Fernandez J.J. 2010. Dinoflagellate polyether within the yessotoxin, pectenotoxin and okadaic acid toxin groups: characterization, analysis and human health implications. Toxicon 56(2): 191-217. doi: 10.1016/j.toxicon.2009.11.005

Echevarria M., Naar J.P., Tomas C., Pawlik J.R. 2012. Effects of Karenia brevis on clearance rates and bioaccumulation of brevetoxins in benthic suspension feeding invertebrates. Aquatic Toxicology 106: 85-94. doi: 10.1016/j.aquatox.2011.10.011

Ekins S., Reschly E.J., Hagey L.R., Krasowski M.D. 2008. Evolution of pharmacologic specificity in the pregnane $\mathrm{X}$ receptor. BMC Evolutionary Biology 8. doi: 10.1186/1471-2148-8-103

Fidler A.E., Holland P.T., Reschly E.J., Ekins S., Krasowski M.D. 2012. Activation of a tunicate (Ciona intestinalis) xenobiotic receptor orthologue by both natural toxins and synthetic toxicants. Toxicon 59: 365-72. doi: 10.1016/j.toxicon.2011.12.008

Gaudon C., Chambon P., Losson R. 1999. Role of the essential yeast protein PSU1 in transcriptional enhancement by the ligand-dependent activation function AF-2 of nuclear receptors. EMBO Journal 18(8): 2229-40. doi: 10.1093/emboj/18.8.2229

Gerssen A., van Olst E.H.W., Mulder P.P.J., de Boer J. 2010. In-house validation of a liquid chromatography tandem mass spectrometry method for the analysis of lipophilic marine toxins in shellfish using matrixmatched calibration. Analytical and Bioanalytical Chemistry 397(7): 307988. doi: 10.1007/s00216-010-3886-2

Gietz R.D., Schiestl R.H. 2007. High-efficiency yeast transformation using the LiAc/SS carrier DNA/PEG method. Nature Protocols 2(1): 31-4. doi: 10.1038/nprot.2007.13 
Gronemeyer H., Gustafsson J.A., Laudet V. 2004. Principles for modulation of the nuclear receptor superfamily. Nature Reviews Drug Discovery 3(11): 950-64. doi: 10.1038/nrd1551

Hashimoto S., Nishimura K., Takahashi K., Itabashi Y. 2011. Evaluation of the possibility that free fatty acids cause false-positive result in diarrhetic shellfish poisoning mouse bioassay in actual use. Food Hygiene and Safety Science 52(3): 194-8. doi: 10.3358/shokueishi.52.194

Humpage A.R., Magalhaes V.F., Froscio S.M. 2010. Comparison of analytical tools and biological assays for detection of paralytic shellfish poisoning toxins. Analytical and Bioanalytical Chemistry 397(5): 1655-71. doi: 10.1007/s00216-010-3459-4

James P., Halladay J., Craig E.A. 1996. Genomic libraries and a host strain designed for highly efficient two-hybrid selection in yeast. Genetics 144(4): 1425-36.

Karimullina E., Li Y., Ginjupalli G.K., Baldwin W.S. 2012. Daphnia HR96 is a promiscuous xenobiotic and endobiotic nuclear receptor. Aquatic Toxicology 116: 69-78. doi: 10.1016/j.aquatox.2012.03.005

Letunic I., Doerks T., Bork P. 2012. SMART 7: recent updates to the protein domain annotation resource. Nucleic Acids Research 40(D1): D302-D5. doi: 10.1093/nar/gkr931

Louvion J.F., Havauxcopf B., Picard D. 1993. Fusion of GAL4-VP16 to a steroid-binding domain provides a tool for gratuitous induction of galactose-responsive genes in yeast. Gene 131(1): 129-34. doi:10.1016/0378-1119(93)90681-R

Lubec G., Afjehi-Sadat L., Yang J.W., John J.P.P. 2005. Searching for hypothetical proteins: theory and practice based upon original data and literature. Progress in Neurobiology 77(1-2): 90-127. doi: 10.1016/j.pneurobio.2005.10.001

MacKenzie A.L. 2013. Biochemistry of digestion and algal biotoxin metabolism in bivalves. Unpublished thesis, University of Otago, Dunedin. 235 p.

MacKenzie L.A., Smith K.F., Rhodes L.L., Brown A., Langi V., Edgar M., Lovell G., Preece M. 2011. Mortalities of sea-cage salmon (Oncorhynchus tshawytscha) due to a bloom of Pseudochattonella verruculosa (Dictyochophyceae) in Queen Charlotte Sound, New Zealand. Harmful Algae 11(0): 45-53. doi: 10.1016/j.hal.2011.07.003

Mansueto V., Cangialsi M.V., Faqi A.S. 2011. Post-embryonic development effect of bisphenol $A$ and tributyltin effects in Ciona intestinalis. Caryologia 64(4): 478-84. doi: 10.1080/00087114.2011.10589815

Matsushima A., Ryan K., Shimohigashi Y., Meinertzhagen I.A. 2013. An endocrine disruptor, bisphenol $A$, affects development in the protochordate Ciona intestinalis: hatching rates and swimming behavior alter in a dose-dependent manner. Environmental Pollution 173: 257-63. doi: 10.1016/j.envpol.2012.10.015

McNabb P.S., Selwood A.I., van Ginkel R., Boundy M., Holland P.T. 2012. Determination of brevetoxins in shellfish by LC/MS/MS: single-laboratory validation. Journal of AOAC International 95(4): 1097-105. doi: 10.5740/jaoacint.11-272

Nicolas J., Hendriksen P.J.M., Gerssen A., Bovee T.F.H., Rietjens I. 2014. Marine neurotoxins: state of the art, bottlenecks and perspectives for mode of action based methods of detection in seafood. Molecular 
Nutrition and Food Research 58(1): 87-100. doi: 10.1002/mnfr.201300520

Norcliffe J.L., Alvarez-Ruiz E., Martin-Plaza J.J., Steel P.G., Denny P.W. 2013. The utility of yeast as a tool for cell-based, target-directed highthroughput screening. Parasitology 141(1): 8-16. doi: $10.1017 / \mathrm{s} 0031182013000425$

Olea N., Pulgar R., Perez P., OleaSerrano F., Rivas A., NovilloFertrell A., Pedraza V., Soto A.M., Sonnenschein C. 1996. Estrogenicity of resinbased composites and sealants used in dentistry. Environmental Health Perspectives 104(3): 298-305. doi: 10.2307/3432888

Pham T.A., Hwung Y.P., Santiso-Mere D., McDonnell D.P., O'Malley B.W. 1992. Ligand-dependent and -independent function of the transactivation regions of the human estrogen receptor in yeast. Molecular Endocrinology 6(7): 1043-50. doi: 10.1210/mend.6.7.1508220

Rastinejad F., Huang P., Chandra V., Khorasanizadeh S. 2013. Understanding nuclear receptor form and function using structural biology. Journal of Molecular Endocrinology 51(3): T1-T21. doi: 10.1530/jme-13-0173

Reguera B., Velo-Suárez L., Raine R., Park M.G. 2012. Harmful dinophysis species: a review. Harmful Algae 14(0): 87-106. doi:

10.1016/j.hal.2011.10.016

Reschly E.J., Bainy A.C.D., Mattos J.J., Hagey L.R., Bahary N., Mada S.R., Ou J., Venkataramanan R., Krasowski M.D. 2007. Functional evolution of the vitamin $\mathrm{D}$ and pregnane $\mathrm{X}$ receptors. BMC Evolutionary Biology 7: 222. doi: $10.1186 / 1471-2148-7-222$

Roje-Busatto R., Ujević I. 2014. PSP toxins profile in ascidian Microcosmus vulgaris (Heller, 1877) after human poisoning in Croatia (Adriatic Sea). Toxicon 79(0): 28-36. doi: 10.1016/j.toxicon.2013.12.014

Routledge E.J., Sumpter J.P. 1996. Estrogenic activity of surfactants and some of their degradation products assessed using a recombinant yeast screen. Environmental Toxicology and Chemistry 15(3): 241-8. doi: 10.1002/etc.5620150303

Schultz J., Milpetz F., Bork P., Ponting C.P. 1998. SMART, a simple modular architecture research tool: identification of signaling domains. PNAS 95(11): 5857-64. doi: 10.1073/pnas.95.11.5857

Sekiguchi K., Sato S., Kaga S., Ogata T., Kodama M. 2001. Accumulation of paralytic shellfish poisoning toxins in bivalves and an ascidian fed on Alexandrium tamarense cells. Fisheries Science 67(2): 301-5. doi: 10.1046/j.1444-2906.2001.00228.x

Selwood A.I., Wilkins A.L., Munday R., Shi F., Rhodes L.L., Holland P.T. 2013. Portimine: a bioactive metabolite from the benthic dinoflagellate Vulcanodinium rugosum. Tetrahedron Letters 54(35): 4705-7. doi: 10.1016/j.tetlet.2013.06.098

Stewart I., McLeod C. 2014. The laboratory mouse in routine food safety testing for marine algal biotoxins and harmful algal bloom toxin research: past, present and future. Journal of AOAC International 97(2): 356-72. doi: 10.5740/jaoacint.SGEStewart

Stewart M., Olsen G., Hickey C.W., Ferreira B., Jelic A., Petrovic M., Barcelo D. 2014. A survey of emerging contaminants in the estuarine receiving environment around Auckland, New Zealand. Science of the Total Environment 468: 202-10. doi: 10.1016/j.scitotenv.2013.08.039 
Suzuki T., Quilliam M.A. 2011. LC-MS/MS analysis of diarrhetic shellfish poisoning (DSP) toxins, okadaic acid and dinophysistoxin analogues and other lipophilic toxins. Analytical Sciences 27(6): 571-84. doi: 0.2116 /analsci.27.571

Suzuki T., Walter J.A., LeBlanc P., MacKinnon S., Miles C.O., Wilkins A.L., Munday R., Beuzenberg V., MacKenzie A.L., Jensen D.J.et al. 2006. Identification of pectenotoxin-11 as 34S-hydroxypectenotoxin-2, a new pectenotoxin analogue in the toxic dinoflagellate Dinophysis acuta from New Zealand. Chemical Research in Toxicology 19(2): 310-8. doi: 10.1021/tx050240y

Traven A., Jelicic B., Sopta M. 2006. Yeast Gal4: a transcriptional paradigm revisited. EMBO Reports 7(5): 496-9. doi: 10.1038/sj.embor.7400679

Vilarino N., Carmen Louzao M., Vieytes M.R., Botana L.M. 2010. Biological methods for marine toxin detection. Analytical and Bioanalytical Chemistry 397(5): 1673-81. doi: 10.1007/s00216-010-3782-9

vom Baur E., Harbers M., Um S.J., Benecke A., Chambon P., Losson R. 1998. The yeast Ada complex mediates the ligand-dependent activation function AF-2 of retinoid X and estrogen receptors. Genes and Development 12(9): 1278-89. doi: 10.1101/gad.12.9.1278

Wang D.Z. 2008. Neurotoxins from marine dinoflagellates: a brief review. Marine Drugs 6: 349-71. doi: 10.3390/md20080016

Wear R.G., Gardner J.P.A. 2001. Biological effects of the toxic algal bloom of February and March 1998 on the benthos of Wellington Harbour, New Zealand. Marine Ecology Progress Series 218: 63-76. doi: 10.3354/meps218063

Welshons W.V., Thayer K.A., Judy B.M., Taylor J.A., Curran E.M., vom Saal F.S. 2003. Large effects from small exposures. I. Mechanisms for endocrine-disrupting chemicals with estrogenic activity. Environmental Health Perspectives 111(8): 994-1006. doi: 10.1289/ehp.5494

Yang Y., Zhou Y., Yu L., Li X., Shi X., Qin X., Rao C., Wang J. 2014. A novel reporter gene assay for Recombinant Human Erythropoietin (rHuEPO) pharmaceutical products. Journal of Pharmaceutical and Biomedical Analysis 100(0): 316-21. doi: 10.1016/j.jpba.2014.08.003

Zhang F., Xu X.X., Li T.T., Liu Z.H. 2013. Shellfish toxins targeting voltagegated sodium channels. Marine Drugs 11(12): 4698-723. doi: $10.3390 / \mathrm{md} 11124698$ 


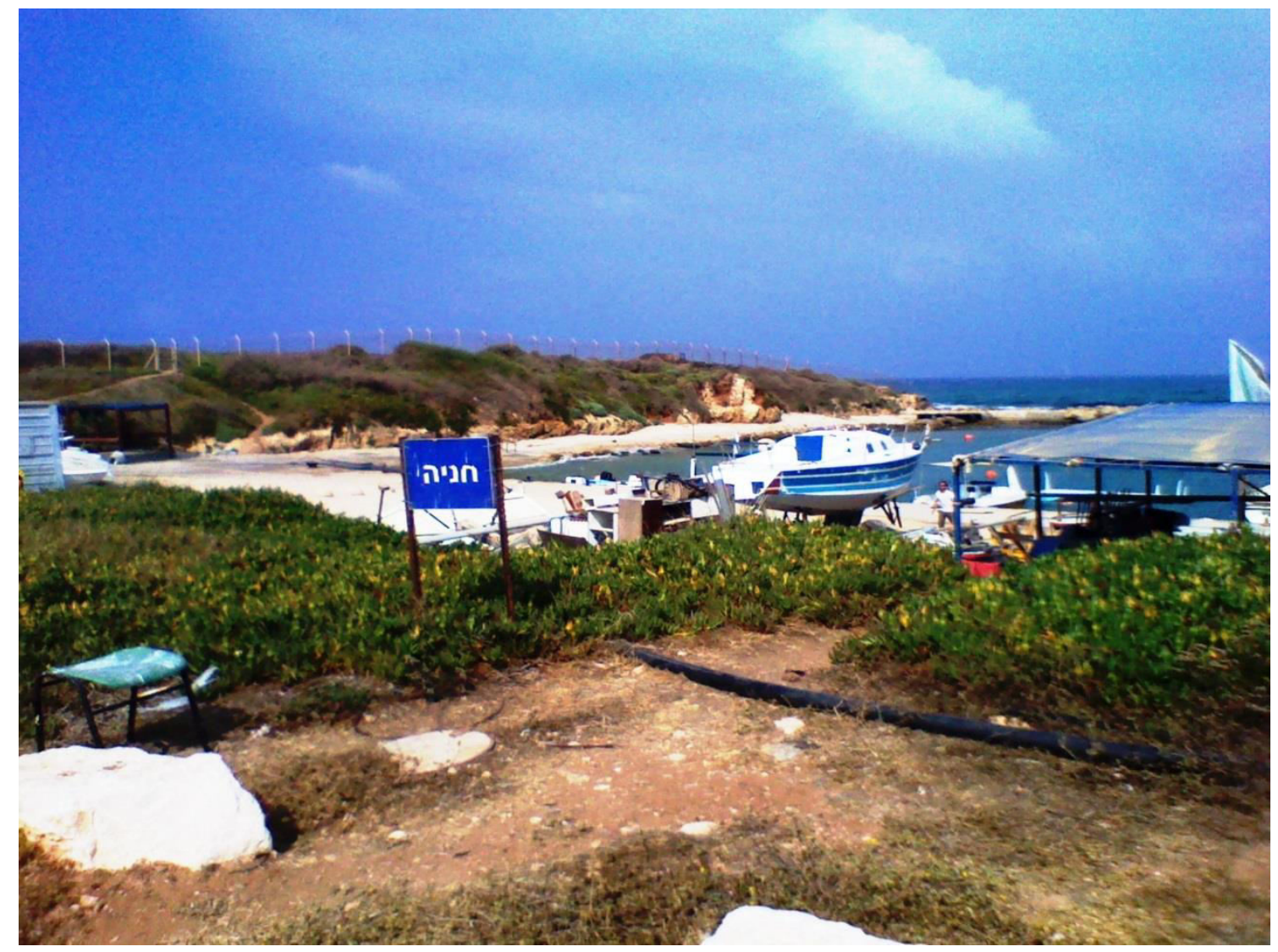

Sampling of Botryllus schlosseri colonies was undertaken from Michmoret beach by the Ruppin Academic Centre, Mediterranean Coast, Israel. 


\section{CHAPTER FOUR}

\section{Utilising tunicate xenobiotic receptors in yeast bioassays for high-throughput screening of bioactive compounds}

\subsection{Introduction}

The development of robust high-throughput screening (HTS) assays for natural marine bioactive compounds for pharmaceutical purposes is technically challenging (Martins et al. 2014). Consequently, despite the enormous number of structurally unique bioactive marine natural products that are known, there are only eight approved drugs, along with 12 natural marine products (or derivatives thereof) in different phases of clinical testing (Mayer et al. 2010; Martins et al. 2014). The development of better bioassays is a key factor for identifying the activity of bioactive chemicals, because the natural biological activities of putative drug compounds influence their potential medical applications (Imhoff et al. 2011; Martins et al. 2014).

A number of nuclear receptors (NRs) have been used successfully during the early phases of drug discovery (Shi 2007). Among the HTS assays, ligandbinding and cell-based NR transactivation assays (Raucy \& Lasker 2010, 2013) are of particular importance in drug discovery and are being used by a growing number of pharmaceutical companies (Chu et al. 2009; Fahmi \& Ripp 2010). Most of the assays are based on mammalian-derived cell lines and screen for compounds that bind to and/or activate pregnane $X$ receptor (PXR), constitutive androstane receptor (CAR) and aryl hydrocarbon receptor (AhR; Michelini et al. 2010; Raucy \& Lasker 2013). For example, cell-based (HepG2) transactivation luciferase reporter gene assays have been developed to identify both PXR ligands and cytochrome P450 enzyme (e.g. CYP3A4) inducers (Herbst et al. 2009). These assays can be used to identify novel drugs and natural products 
that modulate PXR activity which may aid the prediction of drug-drug interactions and xenobiotic-induced toxicities (Chang \& Waxman 2005).

Despite these applications, mammalian cell lines have significant limitations as they require costly and highly specialised culturing facilities and personnel with advanced laboratory skills (Balaguer et al. 1999). The typical mammalian cell line assays that are used (e.g. MCF-7, HeLa) require several days' of growth in steroid-free serum and 16-hour incubations with test chemicals (Balaguer et al. 1999). In contrast, baker's yeast (Saccharomyces cerevisiae) is a wellestablished eukaryotic expression system that is robust, cost-effective and generally easier/faster to perform than mammalian cell-based assays (Leskinen et al. 2005; Balsiger et al. 2010). Additionally, yeast bioassays can be adapted to microplate formats and automated making them particularly well-suited for HTS (de Almeida et al. 2008).

In Chapter Three, I reported the development of a generic recombinant yeast bioassay capable of detecting marine microalgal biotoxins. These recombinant yeast strains expressed chimeric proteins containing the ligand-binding domains (LBDs) of two tunicate (Ciona intestinalis and Botryllus schlosseri) genes that are orthologues to the vitamin D receptor (VDR) and PXR (VDR/PXRa). These chimeric proteins mediate ligand-dependent transcription of a reporter gene (lacZ) encoding the easily assayed enzyme $\beta$-galactosidase. The aim of this chapter was to identify if tunicate VDR/PXR LBDs were activated by marine bioactive compounds, or their chemically modified analogues. It was hoped that this detection system might be useful for detecting new candidates for drug development. Since many drugs used currently are derived from terrestrial organisms, a range of fungal metabolites and one plant-derived natural product were also tested for their activity in the yeast bioassay. 


\subsection{Materials and methods}

\subsubsection{Compounds tested in the yeast bioassay}

The compounds tested in the yeast bioassay are given in Table 4.1 and their structures in Figure 4.1. The following natural products and their analogues were provided by Prof Margaret Brimble (University of Auckland): gymnodimine, gymnodimine-brominated, gymnodimine-dansylated, gymnodimine-oxidised, paecilospirone, CJ-13-015, CJ-13-102, CJ-13-103, CJ-13-104, CJ-13-108, spirolaxine, spirolaxine-methyl ether, thysanone, (-)-deoxydihydrokalafungin and (+)-deoxykalafungin. Naringin was purchased from Sigma-Aldrich (St. Louis, MO, U.S.A.). All chemicals were dissolved in analytical grade ethanol (Merck, Whitehouse Station, NJ, U.S.A.) to form stock solutions. Serial dilutions were added to the yeast bioassay media at a final ethanol concentration of $1 \%$ $(\mathrm{v} / \mathrm{v})$.

\subsubsection{Bioassay procedure}

The tunicate VDR/PXRa LBD-based yeast bioassays, data analyses and development of recombinant yeast strains were performed as described in Chapter Three (Section 3.2.2-3.2.7). 
A

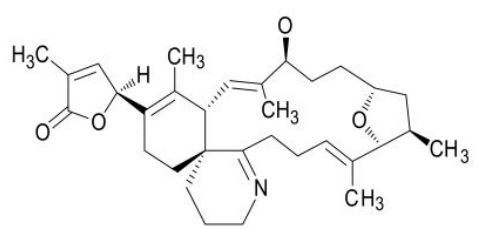

C

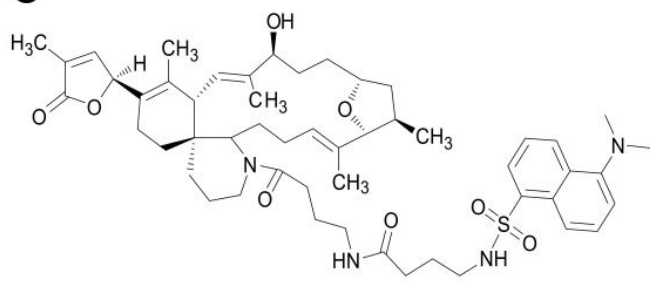

$\mathrm{E}$

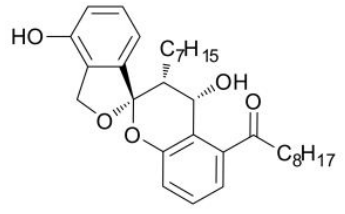

G

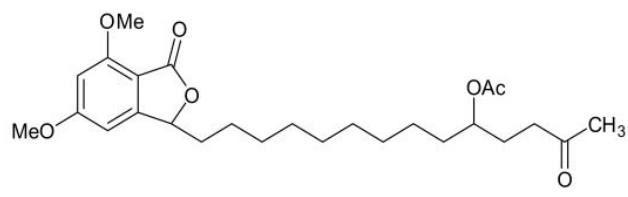

।

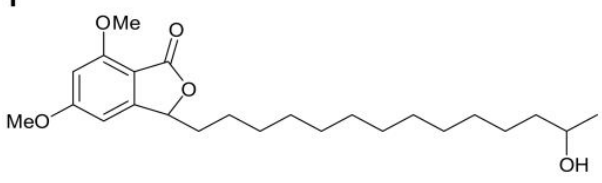

B

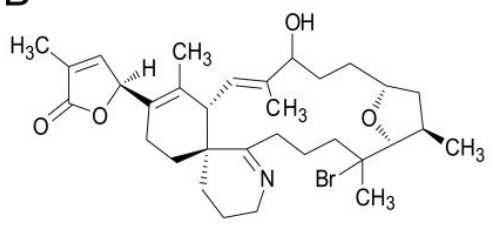

D

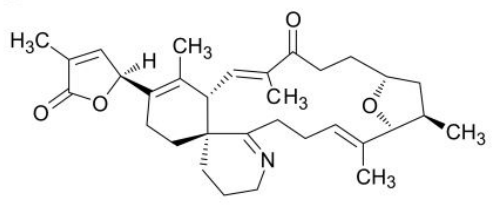

F

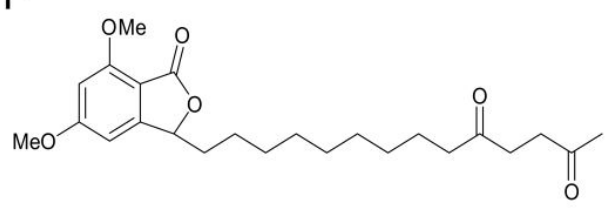

$\mathrm{H}$

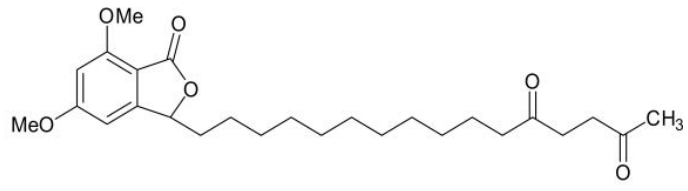

$J$

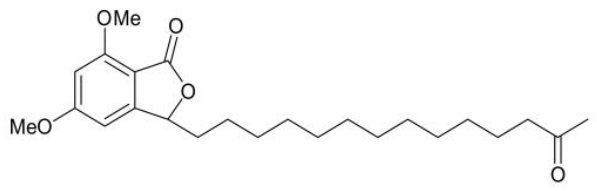

M

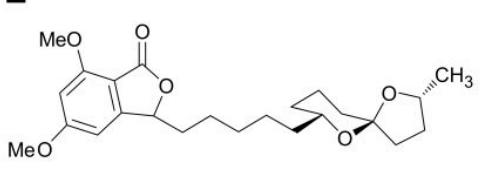

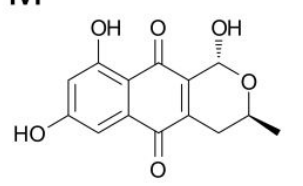

Figure 4.1 Structures of 16 natural products and relevant analogues.

The following chemicals were tested in tunicate VDR/PXRa LBD-based yeast bioassays: (A) gymnodimine, (B) gymnodimine-brominated, (C) gymnodiminedansylated, (D) gymnodimine-oxidised, (E) paecilospirone, (F) CJ-13-015, (G) CJ-13102, (H) CJ-13-103, (I) CJ-13-104, (J) CJ-13-108, (K) spirolaxine, (L) spirolaxinemethyl ether and (M) thysanone. 
N

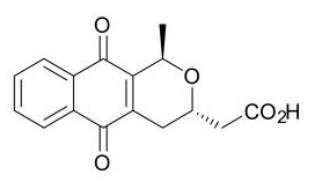

O

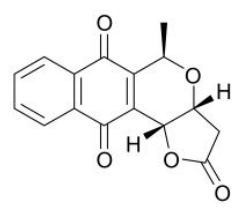

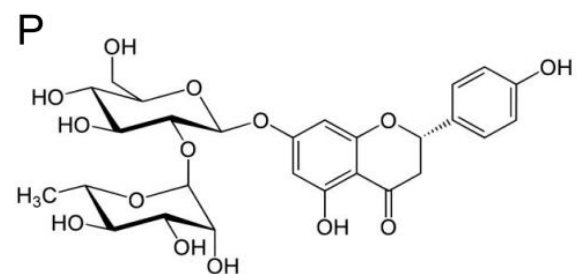

Figure 4.1 (cont.) Structures of 16 natural products and relevant analogues.

The following chemicals were tested in tunicate VDR/PXR $\alpha$ LBD-based yeast bioassays: (N) (-)-deoxydihydrokalafungin, $(\mathbf{O})(+)$-deoxykalafungin and $(\mathbf{P})$ naringin.

\subsection{Results and discussion}

The aim of this study was to test if tunicate VDR/PXRa LBD-based yeast bioassays were activated by a range of structurally diverse natural bioactive compounds sourced from both marine and terrestrial organisms. Of the 16 compounds tested, 12 activated both the $C$. intestinalis and B. schlosseri VDR/PXRa LBD-based yeast bioassays generating $\mathrm{EC}_{50}$ values in the low $\mu \mathrm{M}$ range. A plant-derived natural product, naringin, was particularly potent $\left(E_{50}\right.$ values in the $\mathrm{nM}$ range), while three fungal metabolites and one microalagal biotoxin were inactive (Table 4.1). 
Table 4.1 Activation of the tunicate yeast bioassays by 16 natural products and relevant analogues.

Ligand-dependent induction of $\beta$-galactosidase enzymatic activity was measured in yeast strains carrying plasmids pGAL4.CILBDA31.VP16, pGAL4.CILBD.VP16 or pGAL4.BSLBD.VP16. Mean effective concentrations (EC 50 values) and 95\% confidence intervals $(95 \% \mathrm{Cl})$ are given in $\mu \mathrm{M}$. 95\% confidence intervals represent variation within a triplicate measurement $(\mathrm{n}$ = 3). Coefficients of variance (CV) are given in \% for triplicate intra-plate measurements. All compounds were dissolved in ethanol.

\begin{tabular}{|c|c|c|c|c|c|c|c|}
\hline \multirow[t]{2}{*}{ Chemical name } & \multirow[t]{2}{*}{ Toxin type } & \multicolumn{2}{|c|}{ pGAL4.CiLBD $\triangle 31 . V P 16$} & \multicolumn{2}{|c|}{ pGAL4.CiLBD.VP16 } & \multicolumn{2}{|c|}{ pGAL4.BsLBD.VP16 } \\
\hline & & $\mathrm{EC}_{50}(95 \% \mathrm{Cl})$ & CV & $\mathrm{EC}_{50}(95 \% \mathrm{Cl})$ & CV & $\mathrm{EC}_{50}(95 \% \mathrm{Cl})$ & CV \\
\hline Gymnodimine $^{b}$ & Oral toxicity to mice & $\mathrm{NI}(\mathrm{DNC})$ & DNC & $\mathrm{NI}(\mathrm{DNC})$ & DNC & $\mathrm{NI}(\mathrm{DNC})$ & DNC \\
\hline Gymnodimine-brominated $^{\mathrm{b}}$ & Oral toxicity to mice & $4.8(2.4-9.5)$ & 26 & $4.6(3.5-6.0)$ & 25 & $3.3(2.9-3.7)$ & 22 \\
\hline Gymnodimine-dansylated $^{\mathrm{b}}$ & Oral toxicity to mice & $2.1(1.9-2.2)$ & 18 & $2.0(1.9-2.1)$ & 13 & $1.9(1.9-2.1) \mathrm{v}$ & 35 \\
\hline Gymnodimine-oxidised $^{\mathrm{b}}$ & Oral toxicity to mice & $6.1(2.8-13)$ & 37 & $6.2(3.4-11)$ & 22 & $3.8(2.0-7.1)$ & 21 \\
\hline Paecilospirone ${ }^{b}$ & Anti-cancer activities & $55(18-170)$ & 3 & $15(3.9-55)$ & 4 & $\sim 58$ (DNC) & 46 \\
\hline CJ-13-015 b & Potent antibacterial & $6.1(3.7-10)$ & 23 & $3.7(2.7-4.9)$ & 24 & $3.0(1.7-5.2)$ & 21 \\
\hline CJ-13-102 ${ }^{b}$ & Potent antibacterial & $\sim 6.6$ (DNC) & 425 & $4.7(3.6-6.2)$ & 12 & $2.4(0.4-13)$ & 85 \\
\hline CJ-13-103 ${ }^{a}$ & Potent antibacterial & $8.5(5.7-12)$ & 23 & $15(9.0-25)$ & 35 & $2.1(0.1-42)$ & 40 \\
\hline CJ-13-104 ${ }^{\mathrm{b}}$ & Potent antibacterial & $8.5(2.9-24)$ & 8 & $16(7.6-33)$ & 10 & $20(4.7-82)$ & 6 \\
\hline CJ-13-108 ${ }^{b}$ & Potent antibacterial & $\mathrm{NI}(\mathrm{DNC})$ & DNC & NI (DNC) & DNC & $\mathrm{NI}(\mathrm{DNC})$ & DNC \\
\hline Spirolaxine $^{\mathrm{a}}$ & Potent antibacterial & $\sim 17$ (DNC) & 54 & $18(14-23)$ & 24 & $3.3(0.3-35)$ & 48 \\
\hline
\end{tabular}


Table 4.1 (cont.) Activation of the tunicate yeast bioassays by 16 natural products and relevant analogues.

\begin{tabular}{|c|c|c|c|c|c|c|c|}
\hline \multirow[t]{2}{*}{ Chemical name } & \multirow[t]{2}{*}{ Toxin type } & \multicolumn{2}{|c|}{ pGAL4.CiLBD $\Delta 31 . V P 16$} & \multicolumn{2}{|c|}{ pGAL4.CiLBD.VP16 } & \multicolumn{2}{|c|}{ pGAL4.BsLBD.VP16 } \\
\hline & & $\mathrm{EC}_{50}(95 \% \mathrm{Cl})$ & CV & $\mathrm{EC}_{50}(95 \% \mathrm{Cl})$ & CV & $\mathrm{EC}_{50}(95 \% \mathrm{Cl})$ & CV \\
\hline Spirolaxine-methyl ether $^{a}$ & Potent antibacterial & $33(23-48)$ & 40 & $16(0.5-475)$ & 55 & $21(15-28)$ & 65 \\
\hline Thysanone $^{\mathrm{b}}$ & 3C protease inhibitor & $3.3(2.6-4.1)$ & 13 & $3.0(2.6-3.4)$ & 4 & $2.1(1.7-2.6)$ & 5 \\
\hline (-)-Deoxydihydrokalafungin ${ }^{a}$ & 3C protease inhibitor & $9.7(8.3-11)$ & DNC & $\mathrm{NI}(\mathrm{DNC})$ & DNC & $\mathrm{NI}(\mathrm{DNC})$ & DNC \\
\hline (+)-Deoxykalafungin ${ }^{a}$ & 3C protease inhibitor & $\mathrm{NI}(\mathrm{DNC})$ & DNC & $\mathrm{NI}(\mathrm{DNC})$ & DNC & $\mathrm{NI}(\mathrm{DNC})$ & DNC \\
\hline Naringin ${ }^{b}$ & CYP inhibitor & $108(56-208)^{c}$ & 25 & $67(47-93)^{c}$ & 9 & $140(92-211)^{c}$ & 25 \\
\hline
\end{tabular}

Abbreviations: DNC, did not compute; NI, no induction of $\beta$-galactosidase enzymatic activity; CYP, cytochrome P450.

${ }^{\text {a }}$ Strains were incubated for 24 hours.

${ }^{b}$ Strains were incubated for 48 hours.

${ }^{c} \mathrm{EC}_{50}$ and $95 \% \mathrm{Cl}$ values for naringin are given in $\mathrm{nM}$. 


\subsubsection{Activity of microalgal biotoxins in the yeast bioassays}

Gymnodimine, produced by the dinoflagellate Karenia selliformis, did not induce $\beta$-galactosidase activity in any of the yeast bioassays (Table 4.1, Figure 4.2A). The inactivity of gymnodimine is consistent with a study showing inactivity of gymnodimine in mammalian cell lines expressing the $C$. intestinalis VDR/PXRa LBD (Fidler et al. 2012). The chemically similar gymnodimine analogues (gymnodimine-brominated, -dansylated and -oxidised) induced a response in yeast strains carrying plasmids pGAL4.CIBDA31.VP16, pGAL4.CILBD.VP16 and pGAL4.BsLBD.VP16 (Table 4.1, Figure 4.2B-D) suggesting that structural changes alter activation of the tunicate VDR/PXRa LBD. The coefficients of variance (CV) for these four compounds ranged from 13-37\% (Table 4.1). The increased assay variability (CVs $>25 \%$ ) can be attributed to shallow concentration-dependent response curves as indicated by the $\beta$-galactosidase plateau levels (Figure 4.2B-D).

The response of tunicate VDR/PXRa LBD-based yeast bioassays to different microalgal biotoxins may be due to changes in the physicochemical properties of these compounds. The dansylated gymnodimine analogue, which carries a large dansyl amide side chain (Figure 4.1C), was more potent than the oxidised- and brominated-gymnodimine analogues as indicated by the plateau levels of $\beta$-galactosidase production (Figure 4.2B-D). Previous pharmacophore modelling indicated that there were differences in hydrophobicity, molecular weight and the number of hydrogen bond acceptors/donors between microalgal biotoxins that activate the $C$. intestinalis VDR/PXR LBD (e.g. pectenotoxin-2 and okadaic acid) and those that are inactive (e.g. gymnodimine and yessotoxin; Fidler et al. 2012).

The differential activation of the yeast bioassay by the four gymnodimine analogues is in contrast to a previous study reporting that gymnodimine and its chemically modified analogues (gymnodimine-acetate, gymnodimine-methyl carbonate and gymnodamine) have the same effect on cellular viability of Neuro2a neuroblastoma cell lines (Dragunow et al. 2005). This difference may be attributed to the different mechanisms the bioassays use to measure a response. While a lethal endpoint is measured in the Neuro2a neuroblastoma 
cell line assay, the tunicate VDR/PXRa LBD yeast bioassay measures PXRligand interaction through a physiological process. The inactivity of gymnodimine in the yeast bioassay may be explained by the inability of gymnodimine to bind to PXR orthologues in general, since it was also inactive in mammalian cell line assays expressing human PXR, zebrafish PXR, $C$. intestinalis FXR and $C$. intestinalis LXR (Fidler et al. 2012). This highlights the potential of using PXR LBD-based yeast bioassays, such as the tunicate VDR/PXRa yeast bioassay, to detect bioactive compounds that represent precursors (e.g. gymnodimine analogues) of the active toxin (e.g. gymnodimine; Dragunow et al. 2005). 

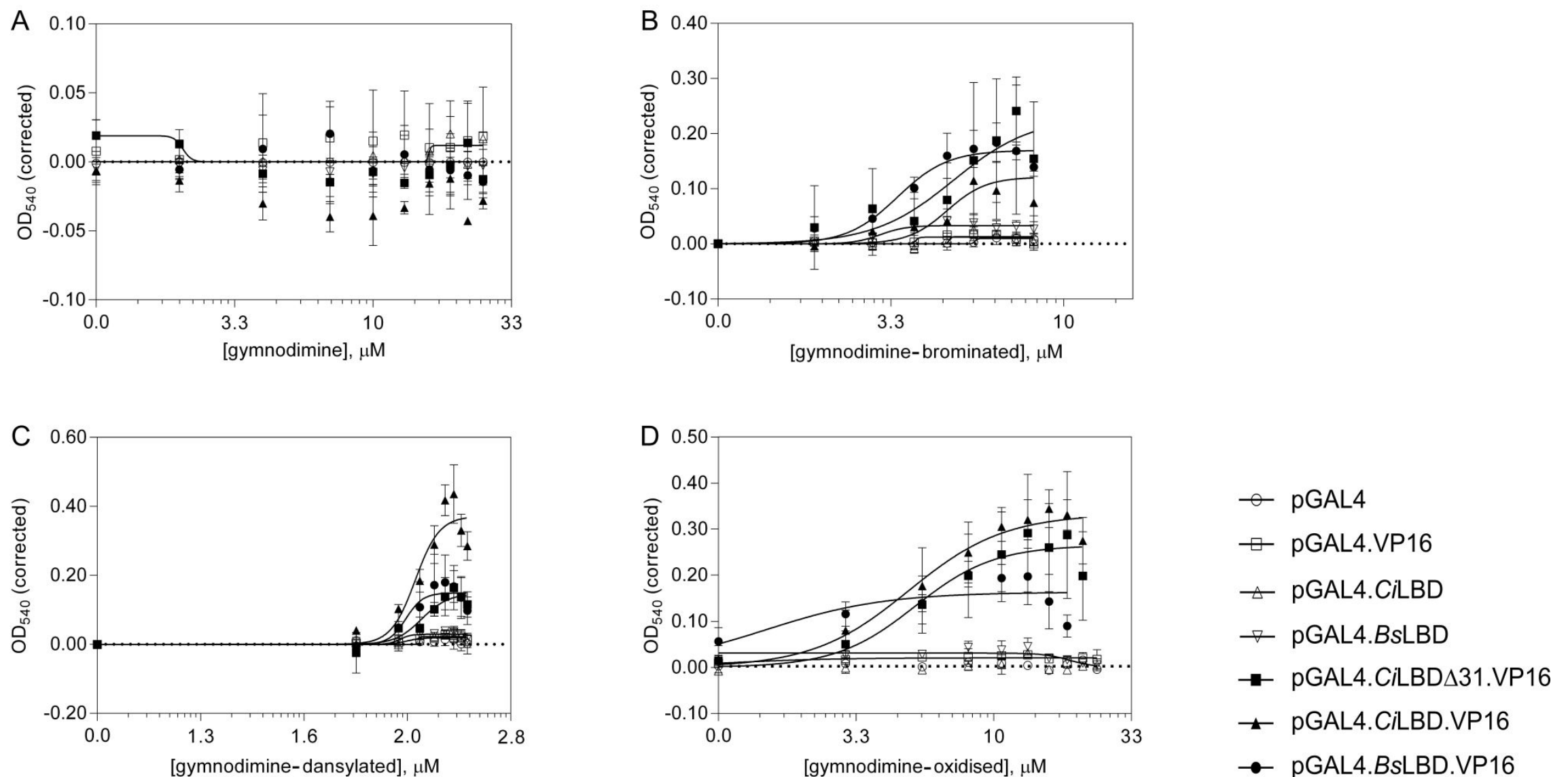

Figure 4.2 Activities of gymnodimine and related analogues tested in the yeast bioassays.

Responses of yeast strains carrying one of seven different pGBKT7-based expression vectors during exposure to a range of concentrations of: (A) gymnodimine, (B) gymnodimine-brominated, (C) gymnodimine-dansylated and (D) gymnodimine-oxidised. $\beta$ -

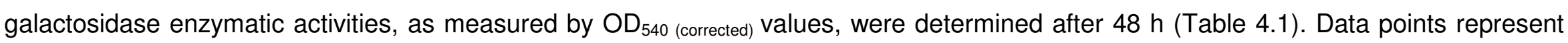
means of $n=3$ replicates. Error bars show \pm one standard deviation. Dotted lines indicate baseline. 


\subsubsection{Activity of fungal metabolites in the yeast bioassays}

The marine fungal metabolite, paecilospirone, was tested in the yeast bioassay to assess whether tunicate VDR/PXR $\alpha$ LBDs were activated by marine natural products other than microalgal biotoxins. Paecilospirone, a [5,6]bisbenzannulated spiroacetal, was first isolated from a marine-derived fungus (Paecilomyces sp.) collected in tropical and sub-tropical coral reef environments (Namikoshi et al. 2000a, b). The yeast strain carrying plasmid pGAL4.CLBD.VP16 showed sigmoidal concentration-dependent $\beta$ galactosidase activity, while responses for strains carrying plasmids pGAL4.ClBBD $31 . V P 16$ and pGAL4.BsLBD.VP16 were non-sigmoidal (Figure 4.3A). The strain expressing the $C$. intestinalis VDR/PXRa LBD, pGAL4.CLBD.VP16, was more strongly activated by paecilospirone than the strain expressing the $B$. schlosseri VDR/PXRa LBD which showed high variability (Table 4.1, Figure 4.3A). Since paecilospirone is a promising lead compound for the development of chemotherapeutic drugs due to its anticancer properties (Sun et al. 2011), these data indicate that tunicate VDR/PXR $\alpha$ LBDs could be used as sensor elements in yeast bioassays for identifying other compounds with similar activities.

In addition to marine fungal metabolites, natural products derived from terrestrial fungi have been an excellent source of pharmaceuticals, for example antibacterial penicillin, cholesterol-lowering lovastatin, antifungal echinocandin B and immunosuppressive cyclosporin A (Evidente et al. 2014). A small group of bioactive fungal metabolites (CJ-13-015, CJ-13-102, CJ-13-103, CJ-13-104, CJ-13-108, spirolaxine and spirolaxine-methyl ether) has been examined previously for their inhibitory activity against the microaerophilic Gram-negative bacterium Helicobacter pylori (Dekker et al. 1997; Radcliff et al. 2008). Over $50 \%$ of the human population are infected by $\mathrm{H}$. pylori, which has been associated with increased risk of developing gastric cancer (Montecucco \& de Bernard 2003). Four CJ-13 analogues (CJ-13-015, -102, -103 and -104) activated the tunicate VDR/PXRa LBDs (Table 4.1, Figure 4.3B-E) suggesting that the yeast bioassay could be useful for identifying other compounds with antibacterial activity. The $B$. schlosseri VDR/PXRa LBD-based yeast bioassay 
(pGAL4.BsLBD.VP16) was less sensitive than the $C$. intestinalis VDR/PXR $\alpha$ equivalent as indicated by the plateau levels of $\beta$-galactosidase production (Figure 4.3D-E). However, this difference was not reflected in the $\mathrm{EC}_{50}$ values (Table 4.1). In contrast, CJ-13-108, was inactive in the yeast bioassay (Table 4.1 , Figure $4.3 \mathrm{~F}$ ), which may be attributed to its limited solubility in organic solvents (Radcliff et al. 2008).

Yeast strains expressing the full-length LBD of the $C$. intestinalis and $B$. schlosseri VDR/PXRa receptors were activated by spirolaxine, while the response of yeast strains expressing the truncated $C$. intestinalis receptor (pGAL4.ClBDD31.VP16) were highly variable (Table 4.1, Figure 4.3G). The chemically similar spirolaxine-methyl ether gave positive responses in all three yeast strains, although they were non-sigmoidal and the 95\% confidence interval $(95 \% \mathrm{Cl})$ for strain pGAL4.CIBDD.VP16 was very wide (Table 4.1, Figure $4.3 \mathrm{H})$.

Yeast strains carrying plasmids pGAL4.CLBD $\Delta 31$.VP16, pGAL4.ClBDD.VP16, or pGAL4.BsLBD.VP16 showed sigmoidal concentration-dependent response curves when tested with the clinically important fungal metabolite, thysanone (Table 4.1, Figure 4.3I). The B. schlosseri VDR/PXRa LBD-based yeast bioassay (pGAL4.BsLBD.VP16) was less sensitive than the two $C$. intestinalis VDR/PXRa LBD-based yeast bioassays (pGAL4.CLBDA31.VP16 and pGAL4.CIBDD.VP16; Figure 4.3I). This effect was not reflected in $\mathrm{EC}_{50}$ values $\left(E C_{50}=2.1-3.3 \mu \mathrm{M}\right.$; Table 4.1) and these were lower than those reported for the inhibition of the human rhinovirus (HRV) $3 \mathrm{C}$ protease in a small peptide screening assay $\left(\mathrm{IC}_{50}=47 \mu \mathrm{M}\right.$; Singh et al. 1991). Screening and identification of lead structures as therapeutic agents against HRVs represents an important area of future research, because HRVs are responsible for causing $50-80 \%$ of common colds in humans (Simancas-Racines et al. 2013).

Both (+)-deoxykalafungin and (-)-deoxydihydrokalafungin, chemical analogues of the closely related fungal natural products kalafungin and dihydrokalafungin, showed almost no ligand-dependent induction of $\beta$-galactosidase activity in the tunicate yeast bioassays (Figure 4.3J-K). One exception was a strain expressing the shorter version of the $C$. intestinalis VDR/PXR $\alpha$ LBD (pGAL4.ClBDD31.VP16) that generated an $\mathrm{EC}_{50}$ value of $9.7 \mu \mathrm{M}$. However, 
concentration-response curves indicated reduced $\beta$-galactosidase enzymatic activity at higher concentrations of both (-)-deoxydihydrokalafungin and (+)deoxykalafungin (Figure 4.3J-K). This suppression effect appeared to be an antagonistic effect mediated through the tunicate VDR/PXRa LBD as it is apparent in the other two test strains carrying plasmids pGAL4.CIBDD.VP16 and PGAL4.BSLBD.VP16 and to a lesser degree in negative control strains (Figure 4.3J). The lack of a response from (+)-deoxykalafungin and (-)deoxydihydrokalafungin could be due to their general toxicity to yeast cells. However, the $O_{620}$ values from the yeast bioassays did not support this hypothesis for (-)-deoxydihydrokalafungin (Appendix 15A). In contrast, suppression observed at high concentrations of (+)-deoxykalafungin (Figure $4.3 \mathrm{~K}$ ) may be toxicity induced effects as the $\mathrm{OD}_{620}$ values were lower (Appendix 15B).

A fluorescence resonance energy transfer (FRET) bioassay found that deoxykalafungin inhibited protein kinase $B(A K T)$, a member of the protein kinase $A, G$ and $C$ families (Korwar et al. 2014). The reported $I_{50}$ value $(0.28$ $\mu \mathrm{M})$ from the FRET bioassay is up to two orders of magnitude lower than the $E C_{50}$ values obtained in the yeast bioassay for the range of fungal natural product analogues tested (1.9-33 $\mu \mathrm{M}$; Table 4.1). Thus, it appears that the yeast bioassay is less sensitive at detecting some compounds when compared with bioassays that do not require reporter cell lines (De et al. 2005). This limitation is intrinsic to all assays relying on living cells (and is a major weakness of cell-based bioassays) since test compounds need to cross the cell wall and/or membrane (Lyttle et al. 1992). 

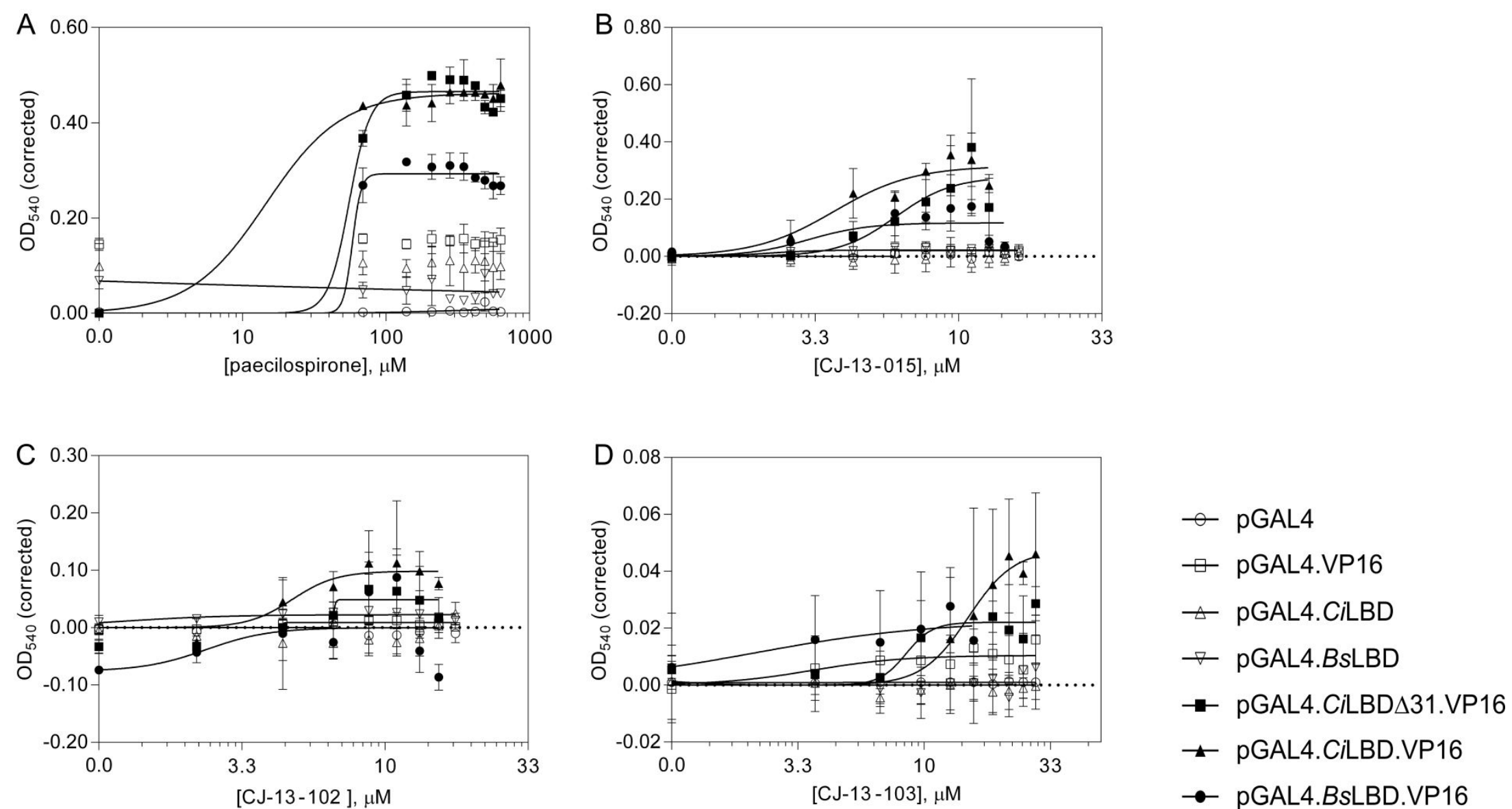

Figure 4.3 Activities of fungal metabolites tested in the yeast bioassays.

Responses of yeast strains carrying one of seven different pGBKT7-based expression vectors during exposure to a range of concentrations of: (A) paecilospirone, (B) CJ-13-015, (C) CJ-13-102 and (D) CJ-13-103. $\beta$-galactosidase enzymatic activities, as

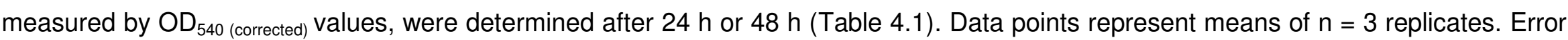
bars show \pm one standard deviation. Dotted lines indicate baseline. 

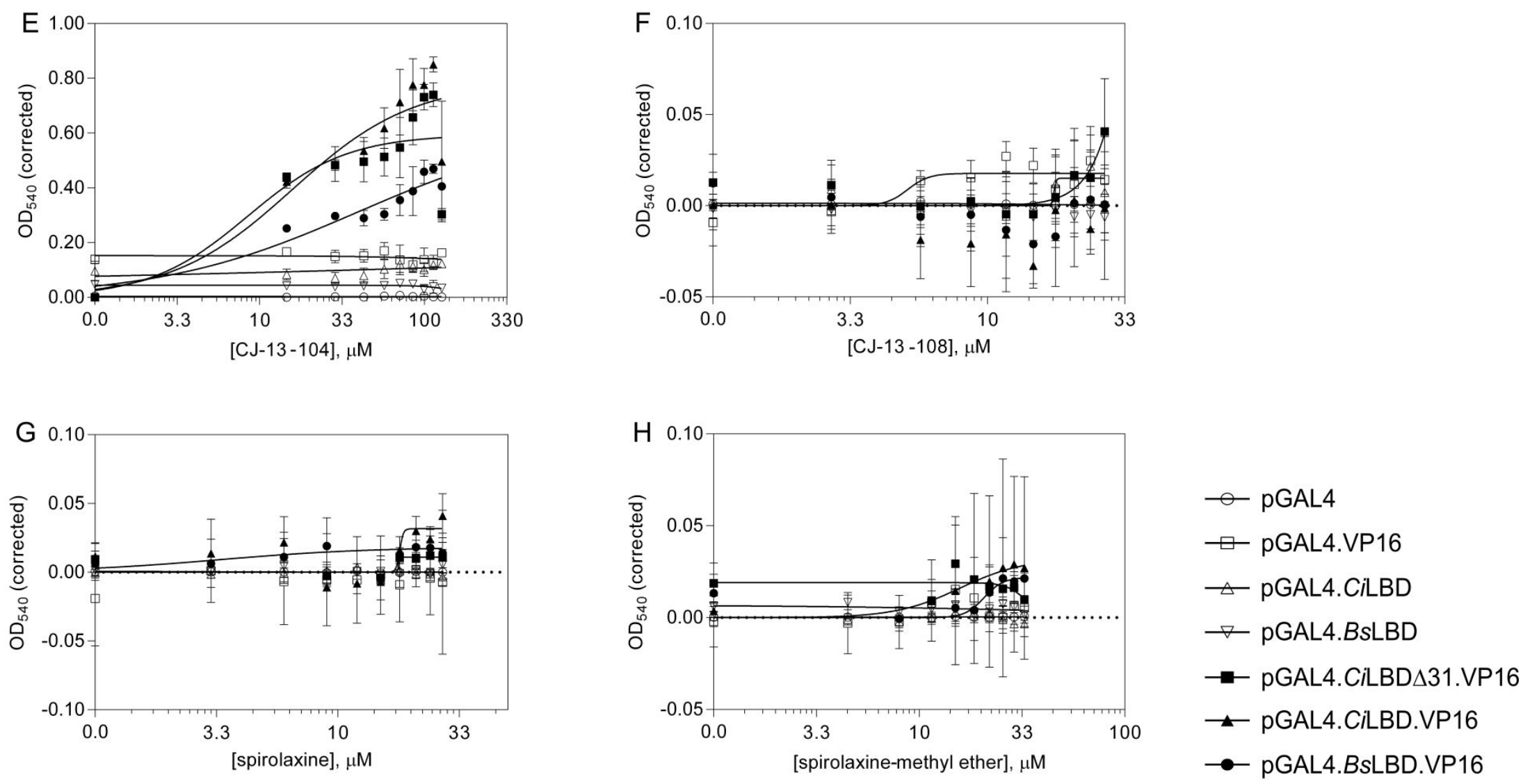

Figure 4.3 (cont.) Activities of fungal metabolites tested in the yeast bioassays.

Responses of yeast strains carrying one of seven different pGBKT7-based expression vectors during exposure to a range of concentrations of: (E) CJ-13-104, (F) CJ-13-108, (G) spirolaxine and $(\mathbf{H})$ spirolaxine-methyl ether. 

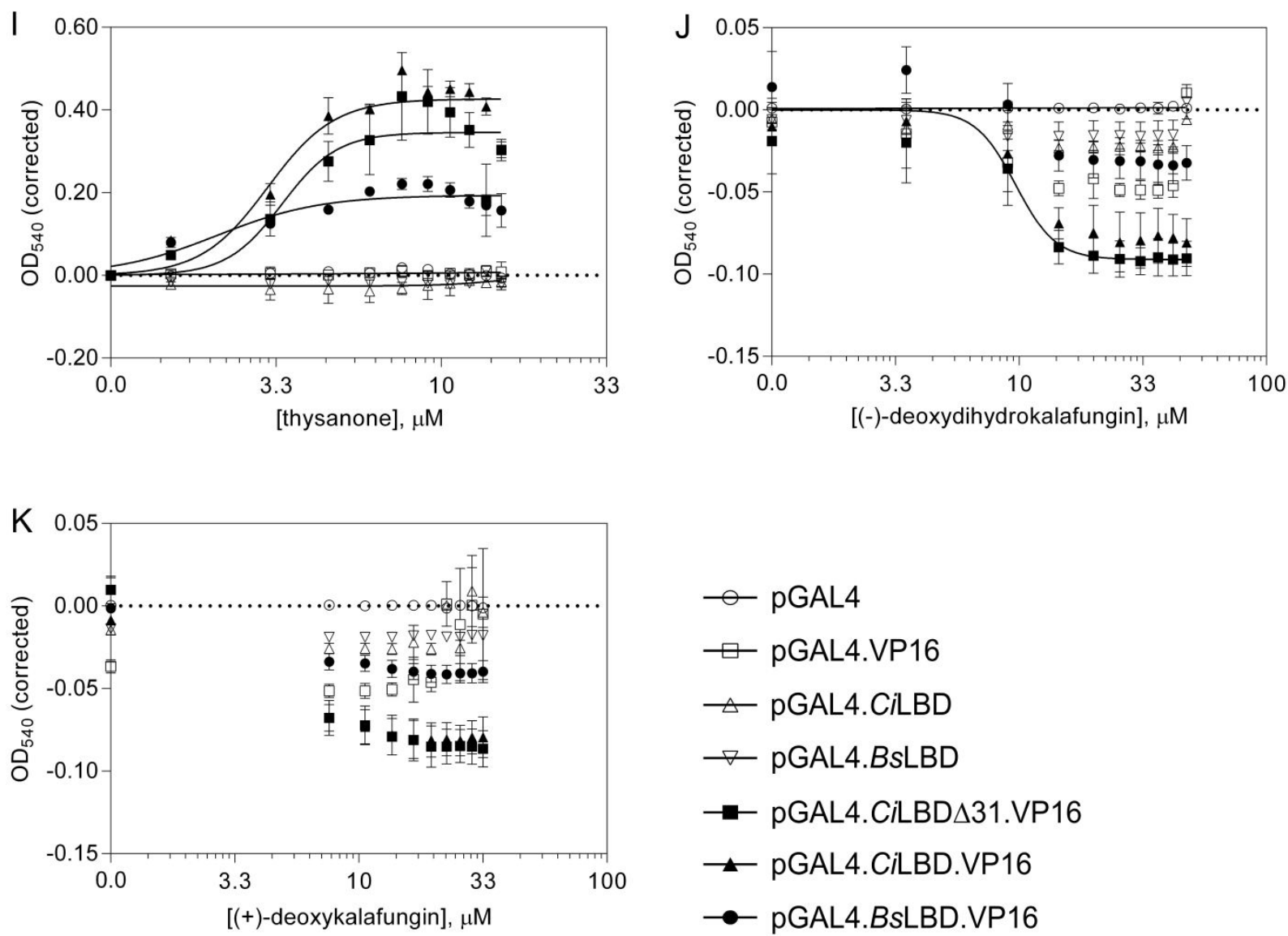

$$
\begin{aligned}
& \multimap \text { pGAL4 } \\
& \square \text { pGAL4.VP16 } \\
& \triangle \text { pGAL4.CiLBD } \\
& \rightarrow \text { pGAL4.BsLBD } \\
& \rightarrow-\text { pGAL4.CiLBD } 31 . V P 16 \\
& \leftarrow \text { pGAL4.CiLBD.VP16 } \\
& \rightarrow \text { pGAL4.BSLBD.VP16 }
\end{aligned}
$$

Figure 4.3 (cont.) Activities of fungal metabolites tested in the yeast bioassays.

Responses of yeast strains carrying one of seven different pGBKT7-based expression vectors during exposure to a range of concentrations of (I) thysanone, (J) (-)deoxydihydrokalafungin and (K) (+)-deoxykalafungin.

\subsubsection{Activity of plant-derived bioactive compounds in the yeast bioassays}

Terrestrial plants produce a plethora of bioactive compounds which are used for drug development (Newman et al. 2003; Cragg et al. 2005; McChesney et al. 2007). The plant-derived flavanone naringin was found to be a potent activator of tunicate VDR/PXRa LBDs expressed in recombinant yeast strains (pGAL4.CIBDA31.VP16, pGAL4.CIBD.VP16 and pGAL4.BSLBD.VP16; Figure 4.4) generating $E_{50}$ values in the low $\mathrm{nM}$ range (Table 4.1). Both $B$. schlosseri and $C$. intestinalis VDR/PXRa LBD-based yeast bioassays showed similar levels of $\beta$-galactosidase enzymatic activity, while the shorter version of the $C$. intestinalis VDR/PXRa LBD (pGAL4.CLBD 31 .VP16) showed a lower 
response as indicated by the plateau levels of $\beta$-galactosidase activity (Figure 4.4). Interestingly, the $\mathrm{EC}_{50}$ values indicated that naringin was two to three orders of magnitude more potent than the other bioactives and derivatives that were tested during this study (Table 4.1). This may be due to structural similarities between flavonoids (e.g. naringin; Figure 4.1P) and the common pharmacophore of $C$. intestinalis VDR/PXRa ligands. Both compounds consist of a planar aromatic structure with hydrophobic regions and multiple hydroxyl groups (Hodek et al. 2002; Ekins et al. 2008; Fidler et al. 2012).

Naringin, the major flavonoid naturally occurring in grapefruit juice, is of particular interest since it exhibits inhibitory effects on the activity of a number of xenobiotic metabolising enzymes (e.g. CYP1A2 and CYP3A4) in vivo (Fuhr 1998; Moon et al. 2006). This inhibitory effect can increase the plasma half-life of many clinically important drugs (Fuhr 1998), which can lead to dangerous drug-drug interactions; such as fatal interactions of azole antifungals with cardiovascular agents (Bates \& Yu 2003; Cascorbi 2012). However, the actual inhibitory effect on a wide range of detoxification enzymes (e.g. CYPs) is not mediated through naringin but through its metabolite, naringenin (Selma et al. 2009), which was not tested during this study. This highlights a limitation of the yeast bioassay. While the tunicate VDR/PXRa yeast bioassay can detect dietary bioactive compounds that are likely to perturb normal physiological functions through interaction with PXR (Dybdahl et al. 2012), metabolic byproducts of these compounds cannot be detected. This is an important shortcoming of all screening assays. They are limited by the compounds tested in these assays, but cannot be used to detect metabolic by-products which are likely to modulate animal biochemistry and physiology differently (Saad et al. 2012). 


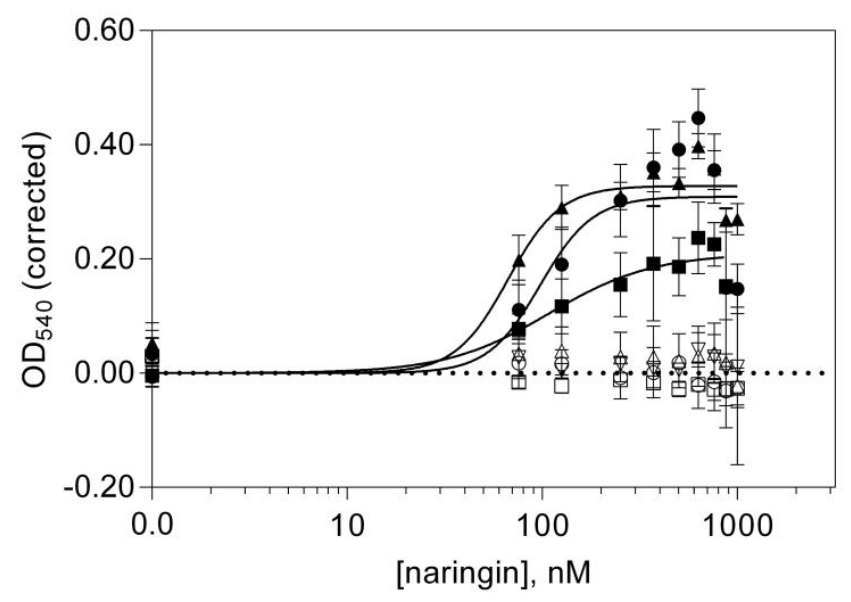

$\multimap$ pGAL4

$\square$ pGAL4.VP16

$\triangle$ pGAL4.CiLBD

$\rightarrow$ pGAL4.BsLBD

$\rightarrow$ pGAL4.CiLBD $\Delta 31$.VP16

$\leftarrow$ pGAL4.CiLBD.VP16

$\longrightarrow$ pGAL4.BsLBD.VP16

Figure 4.4 Activity of naringin tested in the yeast bioassays.

Responses of yeast strains carrying one of seven different pGBKT7-based expression vectors during exposure to a range of concentrations of naringin. $\beta$-galactosidase

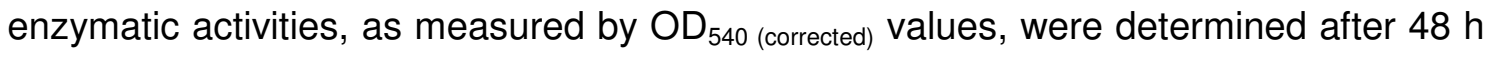
(Table 4.1). Data points represent means of $n=3$ replicates. Error bars show \pm one standard deviation. Dotted lines indicate baseline.

\subsection{Conclusion}

Recombinant yeast strains expressing orthologues of VDR/PXR LBDs from two temperate tunicate species, $C$. intestinalis and B. schlosseri, were utilised for the detection of bioactive compounds. These yeast bioassays were activated by a range of marine natural compounds including microalgal biotoxin analogues and fungal metabolites. Terrestrial fungal bioactive compounds and chemically modified analogues were also successfully detected. Since these compounds provide a rich source of drug leads for pharmaceutical purposes (Molinski et al. 2009; Imhoff et al. 2011), tunicate VDR/PXRa LBDs may represent promising sensor elements for detecting both marine and terrestrial bioactive compounds for drug development. Additionally, the plant-derived flavonoid naringin which can cause dangerous herb-drug interactions in humans (Fuhr 1998) was a potent activator in the tunicate yeast bioassay. 
In this study pure compounds were analysed in the yeast bioassays. This highlights the feasibility of using tunicate VDR/PXRa LBD-based yeast bioassays for detecting bioactive compounds from chemical libraries. Future research should aim to identify medically and/or physiologically active compounds from more complex matrices such as chemical extracts or environmental samples.

Further development of the yeast bioassay into a HTS assay format is particularly important, since this is the most widely used approach for the identification of novel drug lead compounds (Hughes et al. 2011; Martins et al. 2014). The yeast bioassay developed here is particularly well-suited for HTS as it is user-friendly and robust. This is due to the reporter gene lacZ which encodes the enzyme $\beta$-galactosidase. Enzymatic activity can be measured without the need for cell lysis and requires minimal hands-on time. Additionally, assay miniaturisation, one of the main requirements in HTS development, is possible since the nutrient requirements of yeast are easily met in 96-well plate formats (Wolcke \& Ullmann 2001; Hontzeas et al. 2007; Norcliffe et al. 2013). For example, yeast-based luciferase reporter assays for human estrogens, androgens and xenobiotics have been miniaturised from the standard 96-well plate format to high throughput 384- and 1536-well plate formats (Rajasarkka \& Virta 2011).

\subsection{References}

Balaguer P., Francois F., Comunale F., Fenet H., Boussioux A.M., Pons M., Nicolas J.C., Casellas C. 1999. Reporter cell lines to study the estrogenic effects of xenoestrogens. Science of the Total Environment 233(1-3): 4756. doi: 10.1016/S0048-9697(99)00178-3

Balsiger H.A., de la Torre R., Lee W.-Y., Cox M.B. 2010. A four-hour yeast bioassay for the direct measure of estrogenic activity in wastewater without sample extraction, concentration, or sterilization. Science of the Total Environment 408(6): 1422-9. doi: 10.1016/j.scitotenv.2009.12.027

Bates D.W., Yu D.T. 2003. Clinical impact of drug-drug interactions with systemic azole antifungals. Drugs Today 39(10): 801-13. doi: 10.1358/dot.2003.39.10.799473 
Cascorbi I. 2012. Drug interactions- principles, examples and clinical consequences. Deutsches Arzteblatt International 109(33-34): 546-U33. doi: 10.3238/arztebl.2012.0546

Chang T.K., Waxman D.J. 2005. Pregnane X receptor-mediated transcription. Methods in Enzymology 400: 588-98. doi:10.1016/S00766879(05)00033-9

Chu V., Einolf H.J., Evers R., Kumar G., Moore D., Ripp S., Silva J., Sinha V., Sinz M., Skerjanec A. 2009. In vitro and in vivo induction of cytochrome P450: a survey of the current practices and recommendations: a pharmaceutical research and manufacturers of america perspective. Drug Metabolism and Disposition 37(7): 1339-54. doi: 10.1124/dmd.109.027029

Cragg G.M., Kingston D.G.I., Newman D.J. 2005. Anticancer agents from natural products. Boca Raton, U.S.A., CRC Press, Taylor \& Francis Group.

de Almeida R.A., Burgess D., Shema R., Motlekar N., Napper A.D., Diamond S.L., Pavitt G.D. 2008. A Saccharomyces cerevisiae cell-based quantitative beta-galactosidase handling assay compatible with robotic high-throughput screening. Yeast 25(1): 71-6. doi: 10.1002/yea.1570

De S., Macara I.G., Lannigan D.A. 2005. Novel biosensors for the detection of estrogen receptor ligands. Journal of Steroid Biochemistry and Molecular Biology 96(3-4): 235-44. doi: 10.1016/j.jsbmb.2005.04.030

Dekker K.A., Inagaki T., Gootz T.D., Kaneda K., Nomura E., Sakakibara T., Sakemi S., Sugie Y., Yamauchi Y., Yoshikawa N.et al. 1997. CJ-12,954 and its congeners, new anti-Helicobacter pylori compounds produced by Phanerochaete velutina: fermentation, isolation, structural elucidation and biological activities. Journal of Antibiotics 50(10): 833-9. doi: 10.7164/antibiotics.50.833

Dragunow M., Trzoss M., Brimble M.A., Cameron R., Beuzenberg V., Holland P., Mountfort D. 2005. Investigations into the cellular actions of the shellfish toxin gymnodimine and analogues. Environmental Toxicology and Pharmacology 20(2): 305-12. doi: 10.1016/j.etap.2005.02.008

Dybdahl M., Nikolov N.G., Wedebye E.B., Jonsdottir S.O., Niemela J.R. 2012. QSAR model for human pregnane $X$ receptor (PXR) binding: screening of environmental chemicals and correlations with genotoxicity, endocrine disruption and teratogenicity. Toxicology and Applied Pharmacology 262(3): 301-9. doi: 10.1016/j.taap.2012.05.008

Ekins S., Reschly E.J., Hagey L.R., Krasowski M.D. 2008. Evolution of pharmacologic specificity in the pregnane $\mathrm{X}$ receptor. BMC Evolutionary Biology 8. doi: 10.1186/1471-2148-8-103

Evidente A., Kornienko A., Cimmino A., Andolfi A., Lefranc F., Mathieu V., Kiss R. 2014. Fungal metabolites with anticancer activity. Nature Product Reports 31(5): 617-27. doi: 10.1039/c3np70078j

Fahmi O.A., Ripp S.L. 2010. Evaluation of models for predicting drug-drug interactions due to induction. Expert Opinion on Drug Metabolism and Toxicology 6(11): 1399-416. doi: 10.1517/17425255.2010.516251

Fidler A.E., Holland P.T., Reschly E.J., Ekins S., Krasowski M.D. 2012. Activation of a tunicate (Ciona intestinalis) xenobiotic receptor orthologue by both natural toxins and synthetic toxicants. Toxicon 59: 365-72. doi: 10.1016/j.toxicon.2011.12.008 
Fuhr U. 1998. Drug interactions with grapefruit juice- extent, probable mechanism and clinical relevance. Drug Safety 18(4): 251-72. doi: 10.2165/00002018-199818040-00002

Herbst J., Anthony M., Stewart J., Connors D., Chen T., Banks M., Petrillo E.W., Agler M. 2009. Multiplexing a high-throughput liability assay to leverage efficiencies. Assay and Drug Development Technologies 7(3): 294-303. doi: 10.1089/adt.2008.184

Hodek P., Trefil P., Stiborova M. 2002. Flavonoids- potent and versatile biologically active compounds interacting with cytochromes P450. Chemico-Biological Interactions 139(1): 1-21. doi: 10.1016/s00092797(01)00285-X

Hontzeas N., Hafer K., Schiestl R.H. 2007. Development of a microtiter plate version of the yeast DEL assay amenable to high-throughput toxicity screening of chemical libraries. Mutation Research-Genetic Toxicology and Environmental Mutagenesis 634(1-2): 228-34. doi: 10.1016/j.mrgentox.2007.07.001

Hughes J.P., Rees S., Kalindjian S.B., Philpott K.L. 2011. Principles of early drug discovery. British Journal of Pharmacology 162(6): 1239-49. doi: 10.1111/j.1476-5381.2010.01127.x

Imhoff J.F., Labes A., Wiese J. 2011. Bio-mining the microbial treasures of the ocean: new natural products. Biotechnology Advances 29(5): 468-82. doi: 10.1016/j.biotechadv.2011.03.001

Korwar S., Nguyen T., Ellis K.C. 2014. Preparation and evaluation of deconstruction analogues of 7-deoxykalafungin as AKT kinase inhibitors. Bioorganic and Medicinal Chemistry Letters 24(1): 271-4. doi: 10.1016/j.bmcl.2013.11.020

Leskinen P., Michelini E., Picard D., Karp M., Virta M. 2005. Bioluminescent yeast assays for detecting estrogenic and androgenic activity in different matrices. Chemosphere 61(2): 259-66. doi: 10.1016/j.chemosphere.2005.01.080

Lyttle C.R., Damianmatsumura P., Juul H., Butt T.R. 1992. Human estrogen receptor regulation in a yeast model system and studies on receptor agonists and antagonists. Journal of Steroid Biochemistry and Molecular Biology 42(7): 677-85. doi: 10.1016/0960-0760(92)90108-u

Martins A., Vieira H., Gaspar H., Santos S. 2014. Marketed marine natural products in the pharmaceutical and cosmeceutical industries: tips for success. Marine Drugs 12(2): 1066-101. doi: 10.3390/md12021066

Mayer A.M.S., Glaser K.B., Cuevas C., Jacobs R.S., Kem W., Little R.D., McIntosh J.M., Newman D.J., Potts B.C., Shuster D.E. 2010. The odyssey of marine pharmaceuticals: a current pipeline perspective. Trends in Pharmacological Sciences 31(6): 255-65. doi: 10.1016/j.tips.2010.02.005

McChesney J.D., Venkataraman S.K., Henri J.T. 2007. Plant natural products: back to the future or into extinction? Phytochemistry 68(14): 2015-22. doi: 10.1016/j.phytochem.2007.04.032

Michelini E., Cevenini L., Mezzanotte L., Coppa A., Roda A. 2010. Cell-based assays: fuelling drug discovery. Analytical and Bioanalytical Chemistry 398(1): 227-38. doi: 10.1007/s00216-010-3933-z

Molinski T.F., Dalisay D.S., Lievens S.L., Saludes J.P. 2009. Drug development from marine natural products. Nature Reviews Drug Discovery 8(1): 6985. doi: $10.1038 / \mathrm{nrd} 2487$ 
Montecucco C., de Bernard M. 2003. Molecular and cellular mechanisms of action of the vacuolating cytotoxin ( $\mathrm{VacA}$ ) and neutrophil-activating protein (HP-NAP) virulence factors of Helicobacter pylori. Microbes and Infection 5(8): 715-21. doi: 10.1016/S1286-4579(03)00124-2

Moon Y.J., Wang X.D., Morris M.E. 2006. Dietary flavonoids: effects on xenobiotic and carcinogen metabolism. Toxicology in Vitro 20(2): 187210. doi: 10.1016/j.tiv.2005.06.048

Namikoshi M., Kobayashi H., Yoshimoto T., Meguro S. 2000a. Paecilospirone, a unique spiro chroman-2,1 '( 3 'H)-isobenzofuran derivative isolated from tropical marine fungus Paecilomyces sp. Chemistry Letters(4): 308-9. doi: $10.1246 /$ cl.2000.308

Namikoshi M., Kobayashi H., Yoshimoto T., Meguro S., Akano K. 2000b. Isolation and characterization of bioactive metabolites from marinederived filamentous fungi collected from tropical and sub-tropical coral reefs. Chemical and Pharmaceutical Bulletin 48(10): 1452-7. doi: 10.1002/chin.200111230

Newman D.J., Cragg G.M., Snader K.M. 2003. Natural products as sources of new drugs over the period 1981-2002. Journal of Natural Products 66(7): 1022-37. doi: 10.1021/np030096I

Norcliffe J.L., Alvarez-Ruiz E., Martin-Plaza J.J., Steel P.G., Denny P.W. 2013. The utility of yeast as a tool for cell-based, target-directed highthroughput screening. Parasitology 141(1): 8-16. doi: $10.1017 / \mathrm{s} 0031182013000425$

Radcliff F.J., Fraser J.D., Wilson Z.E., Heapy A.M., Robinson J.E., Bryant C.J., Flowers C.L., Brimble M.A. 2008. Anti-Helicobacter pylori activity of derivatives of the phthalide-containing antibacterial agents spirolaxine methyl ether, CJ-12,954, CJ-13,013, CJ-13,102, CJ-13,104, CJ-13,108 and CJ-13,015. Bioorganic and Medicinal Chemistry 16(11): 6179-85. doi: 10.1016/j.bmc.2008.04.037

Rajasarkka J., Virta M. 2011. Miniaturization of a panel of high throughput yeast-cell-based nuclear receptor assays in 384-and 1536-well microplates. Combinatorial Chemistry and High Throughput Screening 14(1): 47-54. doi: 10.2174/1386207311107010047

Raucy J.L., Lasker J.M. 2010. Current in vitro high-throughput screening approaches to assess nuclear receptor activation. Current Drug Metabolism 11(9): 806-14. doi: 10.2174/138920010794328896

Raucy J.L., Lasker J.M. 2013. Cell-based systems to assess nuclear receptor activation and their use in drug development. Drug Metabolism Reviews 45(1): 101-9. doi: 10.3109/03602532.2012.737333

Saad R., Rizkallah M.R., Aziz R.K. 2012. Gut Pharmacomicrobiomics: the tip of an iceberg of complex interactions between drugs and gut-associated microbes. Gut Pathogens 4. doi: 10.1186/1757-4749-4-16

Selma M.V., Espin J.C., Tomas-Barberan F.A. 2009. Interaction between phenolics and gut microbiota: role in human health. Journal of Agricultural and Food Chemistry 57(15): 6485-501. doi: $10.1021 /$ jf902107d

Shi Y. 2007. Orphan nuclear receptors in drug discovery. Drug Discovery Today 12(11-12): 440-5. doi: 10.1016/j.drudis.2007.04.006

Simancas-Racines D., Guerra C.V., Hidalgo R. 2013. Vaccines for the common cold. The Cochrane Database of Systematic Reviews 6: CD002190-CD. doi: 10.1002/14651858.CD002190.pub4 
Singh S.B., Cordingley M.G., Ball R.G., Smith J.L., Dombrowski A.W., Goetz M.A. 1991. Structure of stereochemistry of thysanone: a novel human rhinovirus 3C-protease inhibitor from Thysanophora penicilloides. Tetrahedron Letters 32(39): 5279-82. doi: 10.1016/S00404039(00)92364-5

Sun Z.-L., Zhang M., Zhang J.-F., Feng J. 2011. Antifungal and cytotoxic activities of the secondary metabolites from endophytic fungus Massrison sp. Phytomedicine 18(10): 859-62. doi: 10.1016/j.phymed.2011.01.019 Wolcke J., Ullmann D. 2001. Miniaturized HTS technologies - uHTS. Drug Discovery Today 6(12): 637-46. doi: 10.1016/S1359-6446(01)01807-4 


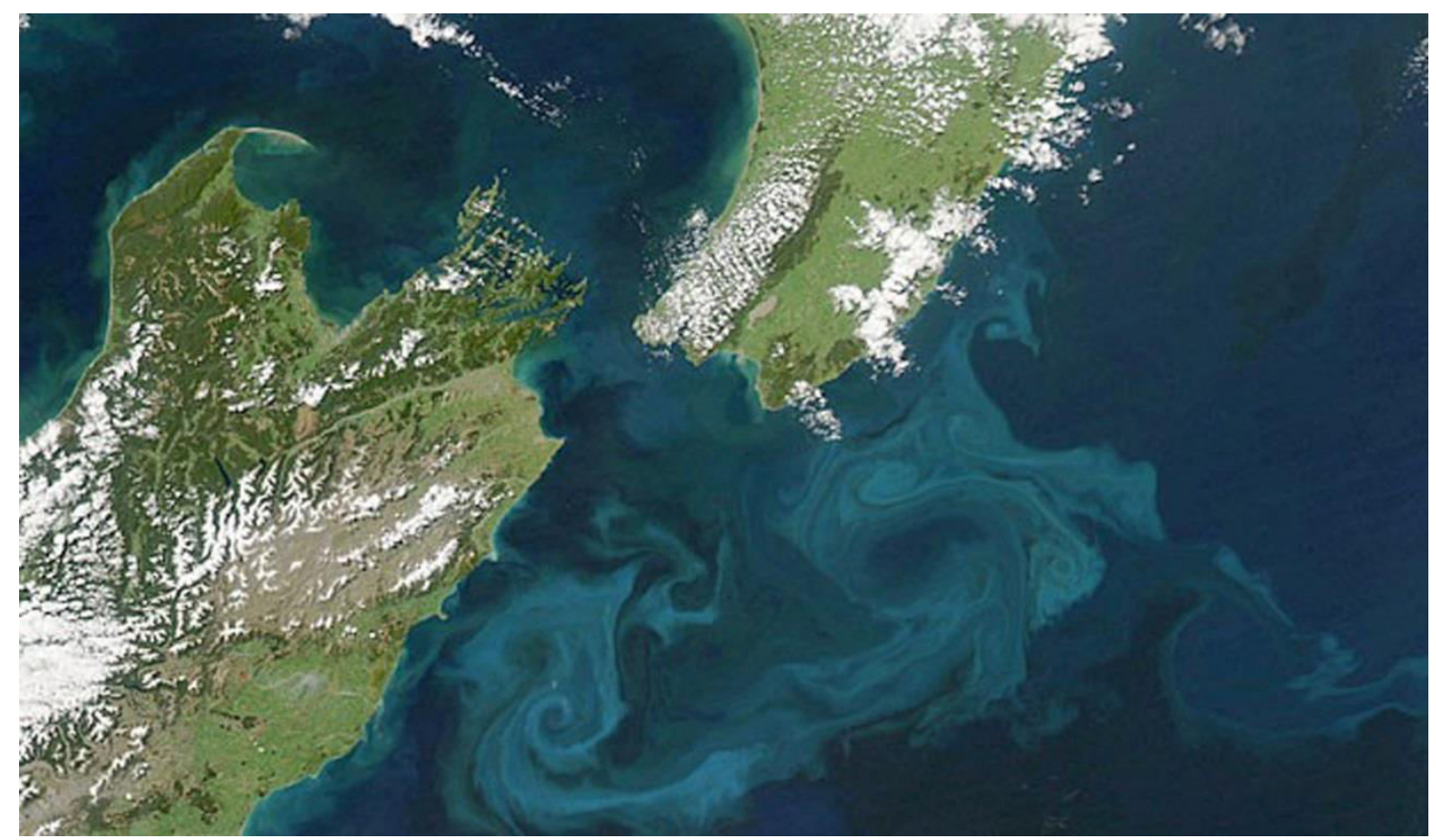

Microalgal bloom observed in Cook Strait, New Zealand. $25^{\text {th }}$ October 2009 Image source: National Aeronautics and Space Administration, U.S.A. 


\section{Utilising tunicate xenobiotic receptors in yeast bioassays for detection of environmental pollutants}

\subsection{Introduction}

Rapid industrial and urban development in the second half of the $20^{\text {th }}$ century has resulted in the emergence of thousands of persistent anthropogenic chemicals (Stewart et al. 2014). These include industrial chemical contaminants which are of environmental concern; i.e. heavy metals (especially zinc, copper and lead), polycyclic aromatic hydrocarbons (PAHs) and pesticides (Järup 2003; Karami-Mohajeri \& Abdollahi 2011; Ball \& Truskewycz 2013). Recently, a new class of non-regulated environmental pollutants, termed emerging contaminants (ECs), has captured scientific and public attention because it may pose a health risk to many organisms, including humans (Gavrilescu et al. 2015). Emerging contaminants are continuously released into the aquatic environment through products such as pharmaceuticals, personal-care products and surfactants (Gavrilescu et al. 2015) and are considered to be 'pseudopersistent' (Daughton 2002). Emerging contaminants generally occur in surface and ground waters as mixtures at concentrations which are far below those thought to be harmful (e.g. $\mathrm{ng} / \mathrm{L}$ to $\mu \mathrm{g} / \mathrm{L}$; Daughton \& Ternes 1999). However, the possibility exists that chronic exposures to these low levels may elicit ecotoxicological effects in many taxa (Fent et al. 2006; Vasquez et al. 2014).

Surfactants are an important class of ECs which have recently been recognised as priority contaminants of concern under the European Commission Water Framework Directive (European Commission 2008). These detergent-like compounds (e.g. dioctyl sodium sulfosuccinate) are the key component of oil dispersants which are used frequently in response to oil spills (Fiocco \& Lewis 1999). Although the acute effects of oil dispersants are low compared to crude oil (Fuller et al. 2004), the large amounts of dispersants used in response to oil 
spills (e.g. approximately $10-100$ ppb was used in the Deepwater Horizon oil spill in the Gulf of Mexico) pose significant health risks to a wide range of marine organisms (Kujawinski et al. 2011; Wise \& Wise 2011; GoodbodyGringley et al. 2013; Almeda et al. 2014).

The recent development of integrated bioanalytical approaches using in vitro bioassays has become a powerful tool to detect and identify bioactive compounds within complex environmental samples (Eggen \& Segner 2003). In vitro reporter gene bioassays based on the mechanism of action for chemicals (e.g. hormone receptor activation) are now commonly used as screening tools for sensitive and specific detection of xenobiotic-like activities in complex samples (Kinani et al. 2010). To date, most attention has been directed towards identifying endocrine-disrupting chemicals (EDCs), because these compounds can disturb the endocrine system via interaction with steroid hormone receptors (Kojima et al. 2010; De Coster \& van Larebeke 2012), such as the estrogen (ER) and androgen receptors (Thomas et al. 2002; Kinani et al. 2010; Colosi \& Kney 2011). Other key nuclear receptors (NRs) such as pregnane $X$ receptor (PXR; Timsit \& Negishi 2007), peroxisome proliferator-activated receptor (Grun \& Blumberg 2009) or glucocorticoid receptor (Odermatt et al. 2006), are also known to be directly activated by environmental contaminants, and have potential to be used as xenobiotic sensors for environmental bioanalytical purposes.

Pregnane $\mathrm{X}$ receptor is of high interest since it is activated by a diverse range of environmental ligands including steroids, pharmaceutical drugs, pesticides and polybromodiethylethers (Jacobs et al. 2005; Lemaire et al. 2006; Sinz et al. 2006). Reporter gene bioassays, based on cultured HeLa cells that permanently express the luciferase reporter gene under the control of a chimeric human PXR, have been developed for the detection of several pesticides (Lemaire et al. 2006), as well as numerous environmental chemicals (i.e. alkylphenols, hormones, pharmaceuticals, pesticides, polychlorinated biphenyls and bisphenol A) in aquatic samples (Creusot et al. 2010; Mnif et al. 2011; Creusot et al. 2013). Due to the extreme number of organic pollutants present in the environment, in silico models (e.g. quantitative structure-activity relationship, QSAR) based on human PXR and other NRs have been developed (Vedani et 
al. 2009; Dybdahl et al. 2012). These models are used for initial screening of compounds before selecting a smaller set of potentially harmful candidates for future in vitro reporter assays. Similar QSAR in silico models are available for various aquatic taxa, although these models are based on experimentally measured in vivo toxicities (Agatonovic-Kustrin et al. 2014; Golbamaki et al. 2014; Singh et al. 2014).

The aim of this chapter was to explore the use of the tunicate VDR/PXR $\alpha$ yeast bioassay (Chapter Three) for the detection of anthropogenic chemicals of environmental relevance. This in vitro bioassay is based on recombinant yeast (Saccharomyces cerevisiae) strains that express chimeric proteins containing the ligand-binding domains (LBDs) of two tunicate PXR orthologues (Ciona intestinalis and Botryllus schlosseri VDR/PXRa) which mediate liganddependent transcription of a reporter gene (lacZ). Thirteen synthetic chemicals were tested in the yeast bioassays. These compounds were selected based on (i) their aromatic, planar structures and hydrophobic features (Ekins et al. 2008) and (ii) their classification as ECs (e.g. pesticides, pharmaceutical drugs and preservatives). In addition, five complex chemical mixtures that are frequently used to mitigate marine oil spills were analysed. The tunicate VDR/PXRa yeast bioassay may provide a reliable and inexpensive tool for environmental monitoring of anthropogenic pollutants.

\subsection{Materials and methods}

\subsubsection{Compounds tested in the yeast bioassay}

The synthetic compounds tested in the yeast bioassay are given in Table 5.1 and their structures in Figure 5.1. All synthetic compounds were purchased from Sigma-Aldrich (St. Louis, MO, U.S.A.) with the following exceptions: ketoconazole (EMD Chemicals, Gibbstown, NJ, U.S.A.), 2-phenylphenol (Acros Organics, Geel, Belgium), radicicol (Cayman Chemical, Ann Arbor, MI, U.S.A) and $n, n$-diethyl-m-toluamide (DEET; Fluka, St. Louis, MO, U.S.A.). All 
compounds were dissolved in analytical grade ethanol (Merck, Whitehouse Station, NJ, U.S.A.; Table 5.1) or dimethyl sulfoxide (DMSO; Sigma-Aldrich; Appendix 16) to form stock solutions (Bovee et al. 2004). Commercial oil dispersants were sourced from within the Cawthron Institute ( $\mathrm{Dr}$ Louis Tremblay) and were diluted in ethanol only (Table 5.2). Serial dilutions were added to the yeast bioassay media at a final solvent concentration of $1 \%(\mathrm{v} / \mathrm{v})$.

\subsubsection{Yeast bioassay procedure}

The tunicate VDR/PXRa LBD-based yeast bioassays, data analyses and development of recombinant yeast strains were performed as described in Chapter Three (Section 3.2.2-3.2.7).

Based on published $\mathrm{EC}_{50}$ values and in vivo toxicity data, a wide concentration range $(0-1 \mathrm{mM})$ was selected for initial analyses of test compounds in the bioassays. Each compound was tested at least twice in separate assays. These data were then used to identify a concentration range that induced $\beta$ galactosidase enzymatic activity in the bioassay while being non-toxic to the yeast cells themselves. Using the identified concentration range, all yeast bioassays were performed in pseudo-triplicates (triplicate measurements within the same assay). Solvents, ethanol or DMSO (final concentration $1 \%(\mathrm{v} / \mathrm{v})$; Bovee et al. 2004), were tested alongside each test compound in each assay. To facilitate intra-plate comparisons, the coefficient of variance (CV) was calculated for each test compound as described in Chapter Three (Section 3.2.7). 


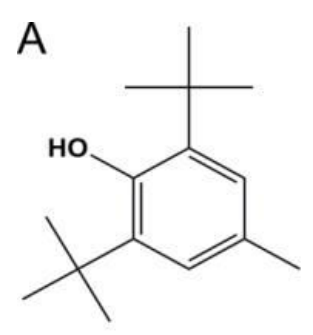<smiles>Cc1cc(O)cc(C)c1Cl</smiles><smiles>Oc1cc(Cl)ccc1Oc1ccc(Cl)cc1Cl</smiles>

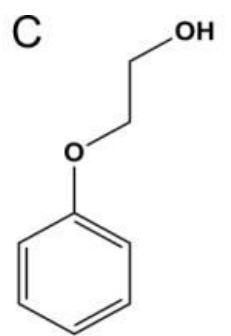<smiles>CCCOC(=O)c1ccc(O)cc1</smiles>

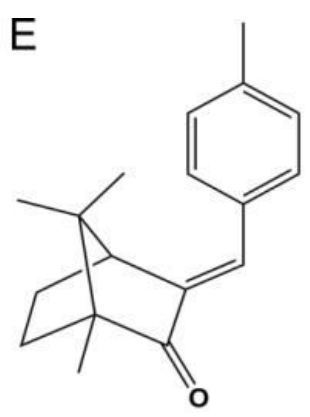<smiles>CCCCC(CC)COC(=O)/C=C/c1ccc(O)cc1</smiles><smiles>O=C(c1ccccc1)c1ccccc1</smiles>

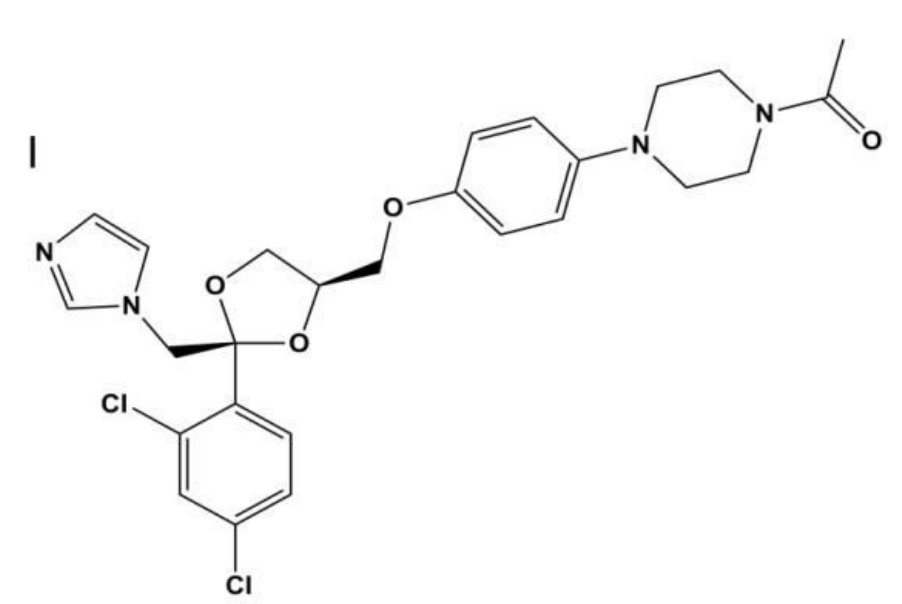

Figure 5.1 Structures of 13 emerging contaminants tested in the yeast bioassays.

The emerging contaminants tested in tunicate VDR/PXRa LBD-based yeast bioassays were: (A) butylated hydroxytoluene, (B) 4-chloro3,5-dimethylphenol, (C) 2-phenoxyethanol, (D) propyl-4-hydroxybenzoate, (E) 4-methylbenzylidene camphor, (F) octyl methoxycinnamate, (G) triclosan, (H) benzophenone and (I) ketoconazole. 
$\mathrm{J}$<smiles>Oc1ccccc1-c1ccccc1</smiles><smiles>[NH3+]OC(=O)Cc1ccccc1Nc1c(Cl)cccc1Cl</smiles>

$\mathrm{K}$<smiles>CC1CC2OC2/C=C/C=C/C(=O)Cc2c(Cl)c(O)cc(O)c21</smiles><smiles>[M]CN(CC)C(=O)c1cccc(C)c1</smiles>

Figure 5.1 (cont.) Structures of 13 emerging contaminants tested in the yeast bioassays.

The emerging contaminants tested in tunicate VDR/PXRa LBD-based yeast bioassays were: (J) 2-phenylphenol, (K) radicicol, (L) diclofenac sodium salt and (M) n,n-diethyl$m$-toluamide (DEET). 


\subsection{Results and discussion}

\subsubsection{Activity of synthetic chemicals in the tunicate yeast bioassays}

The first aim of this study was to explore the potential use of the tunicate VDR/PXRa LBD-based yeast bioassay (Chapter Three) for the detection of synthetic chemicals (e.g. pharmaceuticals, pesticides and fungicides) classified as ECs (Figure 5.1). Of the 13 compounds tested, nine activated both the $C$. intestinalis and $B$. schlosseri VDR/PXRa LBD-based yeast bioassays generating $\mathrm{EC}_{50}$ values in the $\mu \mathrm{M}$ range (Table 5.1).

\subsubsection{Activity of synthetic preservatives}

The four structurally simple synthetic chemicals, 2-phenoxyethanol, 4-chloro3,5-dimethylphenol, propyl-4-hydroxybenzoate and butylated hydroxytoluene (Figure 5.1A-D), are frequently used as preservatives in foods, drugs and cosmetics (Soni et al. 2001; Meyer et al. 2007) and these were active in the yeast bioassays (Table 5.1, Figure 5.2A-D). However, only the antioxidant butylated hydroxytoluene exhibited sigmoidal concentration-response curves (Figure 5.2A) which were consistent across assays (CV $<20 \%$; Table 5.1). Identification of butylated hydroxytoluene as an activator of both tunicate VDR/PXRa LBDs tested $\left(E_{50}=15-30 \mu \mathrm{M}\right.$; Table 5.1) was reasonably consistent with its reported in vivo toxicity towards the colonial tunicate $B$. schlosseri at concentrations $<68 \mu \mathrm{M}$ (Voskoboynik et al. 2002). 
Table 5.1 Activation of the tunicate yeast bioassays by 13 emerging contaminants.

Ligand-dependent induction of $\beta$-galactosidase enzymatic activity was measured in yeast strains carrying plasmids pGAL4.CILBD 431.VP16, pGAL4.CILBD.VP16 or pGAL4.BsLBD.VP16. Mean effective concentrations (EC 50 values) and 95\% confidence intervals $(95 \% \mathrm{Cl})$ are given in $\mu \mathrm{M}$. 95\% confidence intervals represent variation within a triplicate measurement $(\mathrm{n}$ = 3). Coefficients of variance (CV) are given in \% for triplicate intra-plate measurements. All compounds were dissolved in ethanol.

\begin{tabular}{|c|c|c|c|c|c|c|c|c|}
\hline \multirow[t]{2}{*}{ Chemical name } & \multirow[t]{2}{*}{ Toxin type } & \multirow{2}{*}{$\begin{array}{l}\text { Supplier } \\
\text { (Catalogue No.) }\end{array}$} & \multicolumn{2}{|c|}{ pGAL4.CiLBD $\triangle 31 . V P 16$} & \multicolumn{2}{|c|}{ pGAL4.CiLBD.VP16 } & \multicolumn{2}{|c|}{ pGAL4.BsLBD.VP16 } \\
\hline & & & $\mathrm{EC}_{50}(95 \% \mathrm{Cl})$ & CV & $\mathrm{EC}_{50}(95 \% \mathrm{Cl})$ & CV & $\mathrm{EC}_{50}(95 \% \mathrm{Cl})$ & CV \\
\hline $\begin{array}{l}\text { Butylated } \\
\text { hydroxytoluene }^{\text {a }}\end{array}$ & Antioxidant & $\begin{array}{l}\text { Sigma-Aldrich } \\
\text { (B1378) }\end{array}$ & $30(21-42)$ & 20 & $22(20-24)$ & 4 & $15(11-21)$ & 11 \\
\hline $\begin{array}{l}\text { 4-chloro-3,5- } \\
\text { dimethylphenol }^{\text {b }}\end{array}$ & Antimicrobial & $\begin{array}{l}\text { Sigma-Aldrich } \\
\text { (C-4394) }\end{array}$ & $\sim 50$ (DNC) & 77 & $62(26-147)$ & 55 & $25(2.2-286)$ & 41 \\
\hline 2-phenoxyethanol ${ }^{\mathrm{b}}$ & Antimicrobial & Sigma-Aldrich (77699) & $\mathrm{NI}(\mathrm{DNC})$ & DNC & $130(1.2-13,907)$ & 117 & $97(49-193)$ & 154 \\
\hline $\begin{array}{l}\text { Propyl-4-hydroxy- } \\
\text { benzoate }^{\mathrm{b}}\end{array}$ & Antimicrobial & $\begin{array}{l}\text { Sigma-Aldrich } \\
\text { (P53357) }\end{array}$ & $\sim 388$ (DNC) & DNC & $\mathrm{NI}(\mathrm{NI})$ & DNC & $\sim 979$ (DNC) & DNC \\
\hline $\begin{array}{l}\text { 4-methyl-benzylidene } \\
\text { camphor }^{\text {b }}\end{array}$ & EDC & $\begin{array}{l}\text { Sigma-Aldrich } \\
\text { (78551) }\end{array}$ & $140(103-189)$ & 33 & $\sim 125$ (DNC) & DNC & $107(84-137)$ & 6 \\
\hline $\begin{array}{l}\text { Octyl methoxy- } \\
\text { cinnamate }^{b}\end{array}$ & EDC & Sigma-Aldrich (78848) & $20(4.6-87)$ & 30 & $55(5.4-561)$ & 51 & $46(37-58)$ & 19 \\
\hline
\end{tabular}


Table 5.1 (cont.) Activation of the tunicate yeast bioassays by 13 emerging contaminants.

\begin{tabular}{|c|c|c|c|c|c|c|c|c|}
\hline \multirow[t]{2}{*}{ Chemical name } & \multirow[t]{2}{*}{ Toxin type } & \multirow{2}{*}{$\begin{array}{l}\text { Supplier } \\
\text { (Catalogue No.) }\end{array}$} & \multicolumn{2}{|c|}{ pGAL4.CiLBD $\Delta 31 . V P 16$} & \multicolumn{2}{|c|}{ pGAL4.CiLBD.VP16 } & \multicolumn{2}{|c|}{ pGAL4.BsLBD.VP16 } \\
\hline & & & $\mathrm{EC}_{50}(95 \% \mathrm{Cl})$ & CV & $\mathrm{EC}_{50}(95 \% \mathrm{Cl})$ & CV & $\mathrm{EC}_{50}(95 \% \mathrm{Cl})$ & CV \\
\hline Triclosan $^{a}$ & EDC & Sigma-Aldrich (72779) & $318(241-422)$ & 35 & $242(195-299)$ & 11 & $\mathrm{NI}(\mathrm{DNC})$ & DNC \\
\hline Benzophenone ${ }^{\mathrm{b}}$ & EDC & Sigma-Aldrich (B9300) & $221(194-253)$ & DNC & $63(38-108)$ & 30 & $\mathrm{NI}(\mathrm{DNC})$ & DNC \\
\hline Ketoconazole $^{a}$ & Antifungal & $\begin{array}{l}\text { EMD Chemicals } \\
(420600)\end{array}$ & $337(303-375)^{\mathrm{c}}$ & 5 & $400(341-470)^{\mathrm{c}}$ & 18 & $\sim 400(\mathrm{DNC})^{\mathrm{c}}$ & DNC \\
\hline 2-phenylphenol ${ }^{\mathrm{b}}$ & Antifungal & $\begin{array}{l}\text { Acros Organics } \\
(130760050)\end{array}$ & $\mathrm{NI}(\mathrm{DNC})$ & DNC & $79(57-112)$ & 37 & $26(19-35)$ & 18 \\
\hline Radicicol $^{\text {b }}$ & Antifungal & $\begin{array}{l}\text { Cayman Chemical } \\
\text { (13089) }\end{array}$ & $13(9.1-20)$ & 17 & $11(10-12)$ & 9 & $9.5(8.5-11)$ & 9 \\
\hline DEET ${ }^{\mathrm{b}}$ & Insecticide & Fluka (36542) & $99(52-191)$ & 29 & $54(32-89)$ & 34 & $\mathrm{NI}(\mathrm{NI})$ & DNC \\
\hline $\begin{array}{l}\text { Diclofenac sodium } \\
\text { salt }^{\text {b }}\end{array}$ & $\begin{array}{l}\text { Anti- } \\
\text { inflammatory }\end{array}$ & $\begin{array}{l}\text { Sigma-Aldrich } \\
\text { (06899) }\end{array}$ & $91(68-121)$ & 41 & $55(26-121)$ & 44 & $11(0.7-151)$ & 38 \\
\hline
\end{tabular}

Abbreviations: DEET, $n, n$-diethyl-m-toluamide; DNC, did not compute; EDC, endocrine disrupting chemical; NI, no induction of $\beta$-galactosidase enzymatic activity.

${ }^{a}$ Strains were incubated for 24 hours.

${ }^{\mathrm{b}}$ Strains were incubated for 48 hours.

${ }^{\mathrm{c}} \mathrm{EC}_{50}$ and $\mathrm{Cl}$ values for ketoconazole are given in $\mathrm{nM}$. 
Both 2-phenoxyethanol and 4-chloro-3,5-dimethylphenol activated strains carrying plasmids pGAL4.CILBD.VP16 and pGAL4.BSLBD.VP16 although the responses were non-sigmoidal and inconsistent as indicated by very wide $95 \%$ confidence intervals $(95 \% \mathrm{Cl})$ and $\mathrm{CVs}>25 \%$ (Figure 5.2B, C, Table 5.1). It appeared that 2-phenoxyethanol was two orders of magnitude more potent in the tunicate VDR/PXR $\alpha$ LBD-based yeast bioassays $\left(\mathrm{EC}_{50}=97-130 \mu \mathrm{M}\right.$; Table 5.1) when compared to the frog (Xenopus laevis) embryo teratogenesis bioassay $\left(E_{50}=2.5 \mathrm{mM}\right.$; Vrskova \& Modra 2012). However, this comparison is highly tentative due to the considerable variability of $\beta$-galactosidase induction by 2-phenoxyethanol ( $\mathrm{CV}=117-154 \%)$. Activation of both tunicate yeast bioassays by 4-chloro-3,5-dimethylphenol $\left(\mathrm{EC}_{50}=25-62 \mu \mathrm{M}\right.$; Table 5.1) was also highly variable $(\mathrm{CV}=41-554 \%)$ but the values were consistent with previous studies which showed that concentrations $>50 \mu \mathrm{M}$ were highly toxic to fish (Houtman et al. 2004a).

Paraben (propyl-4-hydroxybenzoate) activated strains carrying plasmids pGAL4.CLBD 31 .VP16 and pGAL4.BSLBD.VP16, but there was high variability (Table 5.1). The concentration-response curves were non-sigmoidal and showed a negative effect on the induction of $\beta$-galactosidase activity. This suppression effect was observed in strains carrying the plasmids pGAL4.CLBD $31 . V P 16$ and PGAL4.BsLBD.VP16, but not in the negative control strains (Figure 5.2D). Examination of $\mathrm{OD}_{620}$ values suggests a general toxicity of propyl-4-hydroxybenzoate towards yeast cells (Appendix 17A). In strains carrying plasmids pGAL4.CLBD $\triangle 31$.VP16 and pGAL4.BSLBD.VP16 this toxic effect was most apparent within a concentration range of $50-350 \mu \mathrm{M}$. At concentrations $<350 \mu \mathrm{M}$ the toxic effect decreased which coincided with an increase in $\beta$-galactosidase activity at higher concentrations (Figure 5.2D, Appendix 17A). In contrast, a suppression effect was obtained at high concentrations $(350-500 \mu \mathrm{M})$ in strains carrying plasmid pGAL4.CLBD 31 .VP16 (Appendix 17A), with these strains showing no liganddependent $\beta$-galactosidase enzymatic activity (Figure 5.2D). Despite the considerable assay inconsistencies (Table 5.1), the $C$. intestinalis $\mathrm{EC}_{50}$ value reported in this study $(388 \mu \mathrm{M})$ was comparable to those obtained using acute immobilisation assays with Daphnia magna $\left(\mathrm{EC}_{50}=127 \mu \mathrm{M}\right.$; Terasaki et al. 2009). 
Collectively, the data from the in vitro yeast bioassay and previous in vivo studies (Voskoboynik et al. 2002; Houtman et al. 2004a; Vrskova \& Modra 2012) highlight the high toxicity of these synthetic chemicals towards a wide range of aquatic organisms. This is of particular concern since these compounds are being detected with increasing frequency in aquatic environments (Daughton \& Ternes 1999; Kolpin et al. 2004; Kasprzyk-Hordern et al. 2008; Kimura et al. 2014). 2-phenoxyethanol can cause serious immunogenic and toxic side-effects in humans (Bis \& Mallela 2014), whilst 4chloro-3,5-dimethylphenol, butylated hydroxytoluene and propyl-4hydroxybenzoate are practically non-toxic to mammals including humans (Soni et al. 2001; Houtman et al. 2004a). However, some of these compounds were shown to have weak estrogenic activity when tested in yeast estrogen reporter gene assays and may therefore interrupt normal endocrine functions in animals (Routledge et al. 1998; Miller et al. 2001). 

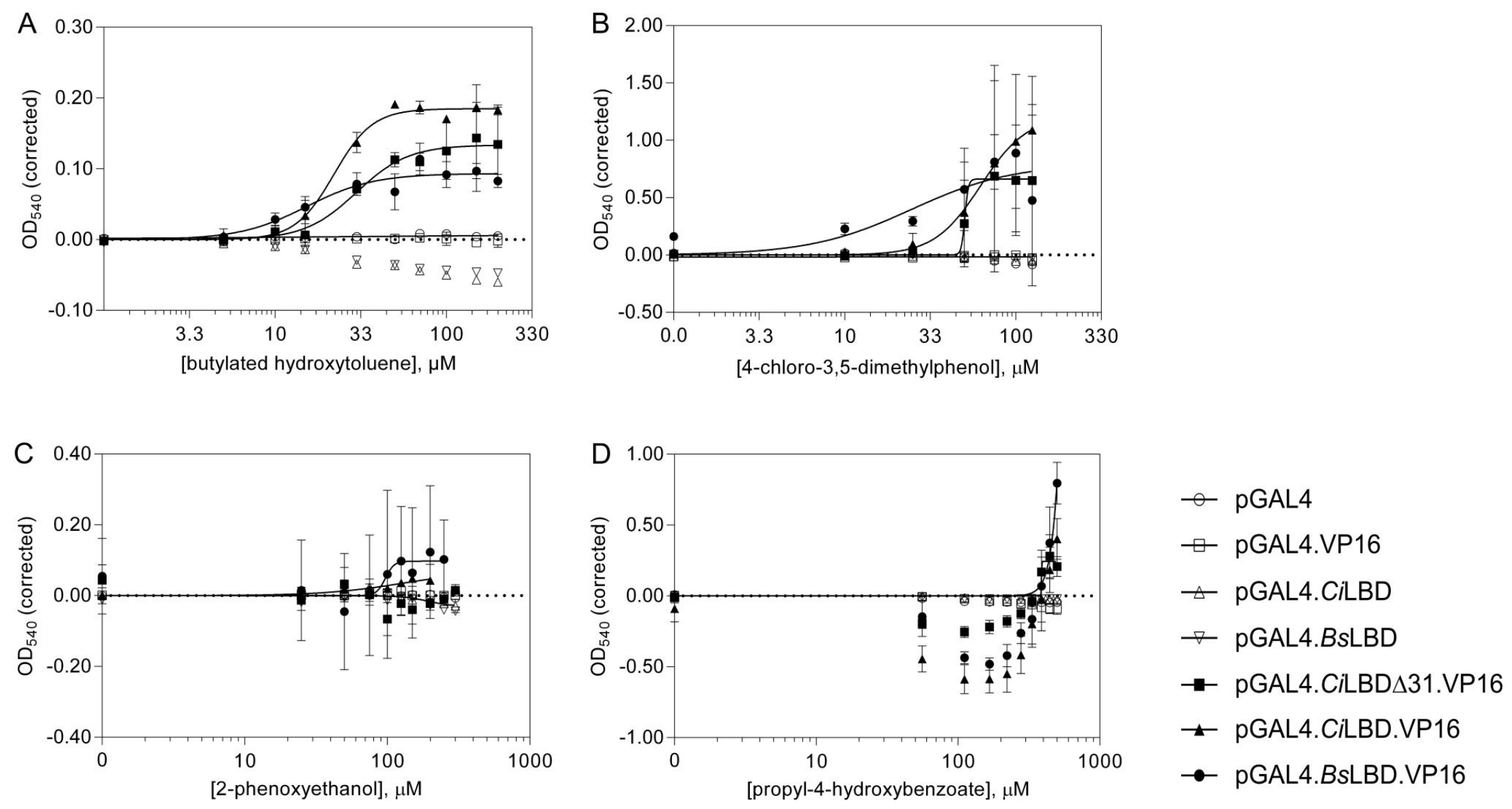

Figure 5.2 Activities of synthetic preservatives tested in the yeast bioassays.

Responses of yeast strains carrying one of seven different pGBKT7-based expression vectors during exposure to a range of concentrations of: (A) butylated hydroxytoluene, (B) 4-chloro-3,5-dimethylphenol, (C) 2-phenoxyethanol and (D) propyl-4hydroxybenzoate. $\beta$-galactosidase enzymatic activities, as measured by $\mathrm{OD}_{540}$ (corrected) values, were determined after $24 \mathrm{~h}$ or $48 \mathrm{~h}$ (Table 5.1). Data points represent means of $n=3$ replicates. Error bars show \pm one standard deviation. Dotted lines indicate baseline. 


\subsubsection{Activity of endocrine disrupting chemicals}

Endocrine disrupting chemicals (EDCs) are of particular concern since they can interfere with hormonal regulation and the normal endocrine system (Kojima et al. 2010; De Coster \& van Larebeke 2012). It is well-known that most EDCs disrupt normal endocrine functions via interaction with sex steroid hormone receptors such as ERa (Routledge \& Sumpter 1996; Houtman et al. 2004b; Hill et al. 2010). Some EDCs may also affect sex steroid hormone receptors indirectly by induction of metabolic enzymes via PXR, leading to disturbance of the endocrine system (Mikamo et al. 2003; Jacobs et al. 2005).

The two organic ultraviolet absorbing chemicals, 4-methylbenzylidene camphor and octyl methoxycinnamate, tested in this study are used widely in personal care products (e.g. sunscreens) and are known to have estrogenic activity (Miller et al. 2001). Both compounds were active in yeast strains carrying plasmids pGAL4.CLBDA31.VP16, pGAL4.CLBD.VP16 and pGAL4.BSLBD.VP16 (Figure 5.3A-B) although the 95\% Cls and CVs of the $E_{50}$ values were very high (Table 5.1). 4-methylbenzylidene camphor has previously been shown to be highly toxic to $D$. magna using an acute toxicity test, with a reported $\mathrm{LC}_{50}$ value of $2.2 \mu \mathrm{M}$ (Fent et al. 2010) being two orders of magnitude lower than the $\mathrm{EC}_{50}$ values observed in this study $\left(E C_{50}=107-140\right.$ $\mu \mathrm{M}$; Table 5.1). Identification of 4-methylbenzylidene camphor and octyl methoxycinnamate as activators of both tunicate VDR/PXRa LBDs (Table 5.1) is consistent with their previously reported anti-estrogenic activities (4methylbenzylidene camphor, $\mathrm{IC}_{50}=87.3 \mu \mathrm{M}$; octyl methoxycinnamate, $\mathrm{IC}_{50}=$ $4.3 \mathrm{mM}$ ) and anti-androgenic activities (4-methylbenzylidene camphor, $\mathrm{IC}_{50}=$ $11.8 \mu \mathrm{M}$; octyl methoxycinnamate, $\mathrm{IC}_{50}=312 \mu \mathrm{M}$ ) using a human $\mathrm{ER \alpha}$ yeast transactivation assay (Gomez et al. 2005; Kunz \& Fent 2006).

Two additional synthetic EDCs (triclosan and benzophenon), with planar structures and at least one hydrogen bond acceptor, were tested in this study (Table 5.1, Figure 5.1G, H). Triclosan was identified as an activator of yeast strains expressing the $C$. intestinalis VDR/PXRa LBD (pGAL4.CIBD $\triangle 31$.VP16 and pGAL4.CLBD.VP16), although this activation was relatively weak as indicated by the plateau levels of $\beta$-galactosidase production (Figure $5.3 \mathrm{C}$ ). In 
strains that express the $B$. schlosseri VDR/PXRa LBD, reduced $\beta$-galactosidase enzymatic activity at higher concentrations was observed (Figure 5.3C). This suppression appeared to be an antagonistic effect mediated through the tunicate VDR/PXRa LBD as it was apparent in all strains that carry a tunicate VDR/PXRa LBD but not in any of the negative control strains (Appendix 17B). Activation of tunicate VDR/PXR orthologues by triclosan $\left(\mathrm{EC}_{50}=242-318 \mu \mathrm{M}\right.$; Table 5.1) is consistent with that reported by Jacobs et al. (2005) who showed that triclosan moderately activates human PXR (46.2\% activity relative to the prototypical inducer rifampicin) when transiently transfected into $\mathrm{HuH} 7$ cell lines.

Benzophenone activated yeast strains carrying plasmids pGAL4.CLBDA31.VP16 and pGAL4.CILBD.VP16 (Table 5.1). However, negative control strains also showed induced $\beta$-galactosidase activity (Figure 5.3D). These results suggest that $\beta$-galactosidase activity is induced by a process that is not dependant on binding of benzophenone to the tunicate VDR/PXRa LBD. In addition, two positive strains (pGAL4.CIBD 31 .VP16 and pGAL4.BSLBD.VP16) showed concentration-dependent reduction of $\beta$ galactosidase activity (Figure 5.3D). Examination of $\mathrm{OD}_{620}$ values indicated a toxic effect towards yeast cells only in one strain (pGAL4.BsLBD.VP16), while all other strains showed normal growth rates at high concentrations of benzophenone (Appendix 17C). The $\mathrm{EC}_{50}$ values obtained from two of the strains (pGAL4.CLBD $31 . V P 16$ and pGAL4.CLBD.VP16) need to be treated with caution as they may reflect a ligand-independent activation (Table 5.1). Identification of benzophenone as a $C$. intestinalis VDR/PXRa ligand is consistent with previous research which showed that benzophenone interacts with vertebrate PXR LBDs (e.g. rat PXR) when using a yeast-two hybrid assay (Mikamo et al. 2003).

Collectively, these results suggest the potential of EDCs to interact with tunicate PXR orthologues. Endocrine disrupting chemicals such as benzophenone, triclosan and 4-methylbenzylidene camphor are important environmental pollutants which are frequently detected in rivers, lakes and coastal areas (Kolpin et al. 2002; Lindstrom et al. 2002; Langford \& Thomas 2008). These environmental contaminants can exhibit their endocrine disruptor activities by altering PXR-regulated steroid hormone metabolism. Therefore, yeast 
bioassays utilising PXR LBDs may represent a valuable tool to detect EDCs with PXR-like activities in the environment (Mikamo et al. 2003). Further research is required to validate the data presented here due to the high variability and limited assay sensitivity to some EDC compounds. 

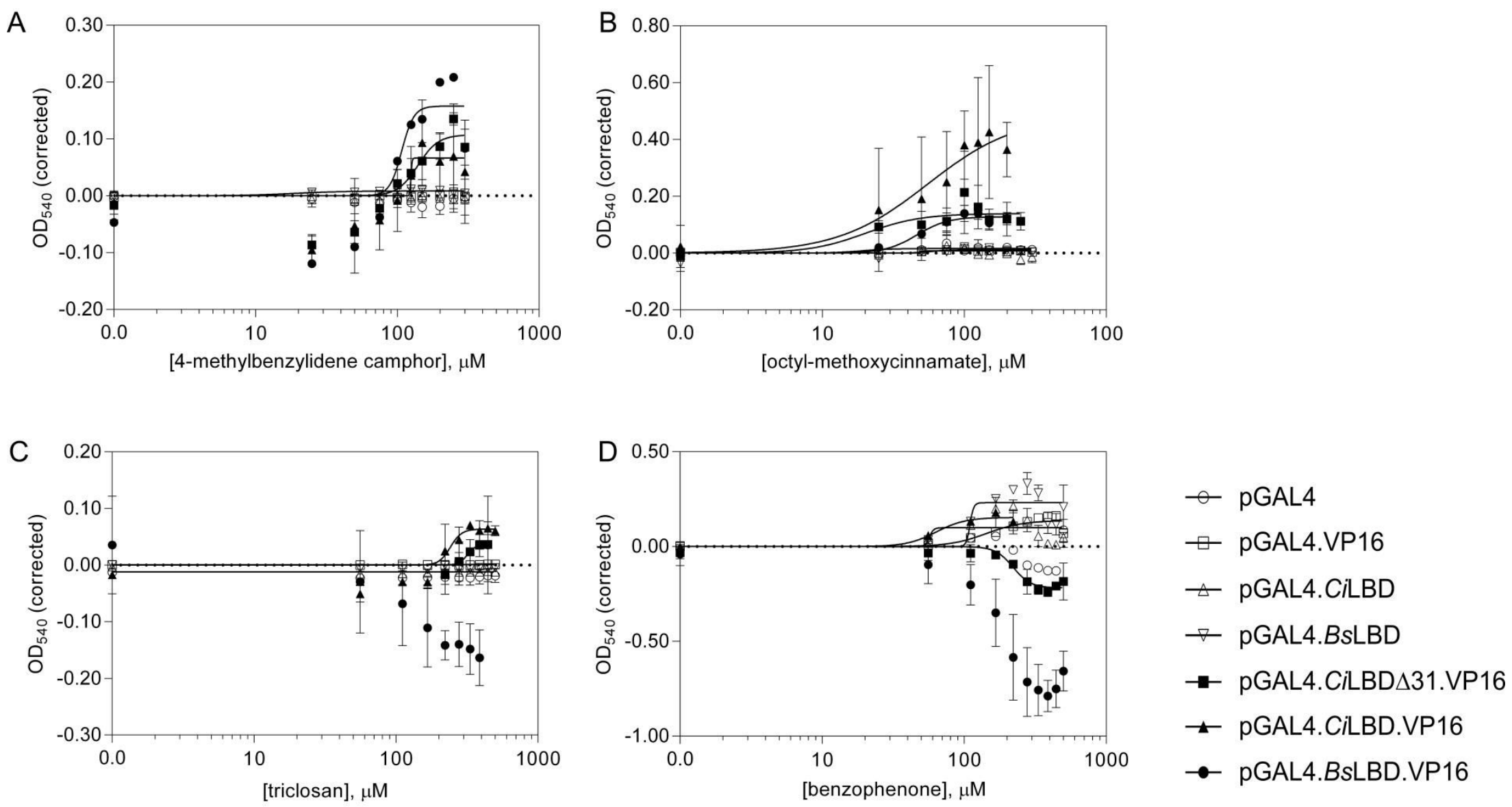

Figure 5.3 Activities of endocrine disrupting chemicals tested in the yeast bioassays.

Responses of yeast strains carrying one of seven different pGBKT7-based expression vectors during exposure to a range of concentrations of: (A) 4-methylbenzylidene camphor, (B) octyl-methoxycinnamate, (C) triclosan and (D) benzophenone. $\beta$-galactosidase enzymatic activities, as measured by $\mathrm{OD}_{540 \text { (corrected) }}$ values, were determined after $24 \mathrm{~h}$ or $48 \mathrm{~h}$ (Table 5.1). Data points represent means of $\mathrm{n}=3$ replicates. Error bars show \pm one standard deviation. Dotted lines indicate baseline. 


\subsubsection{Activity of fungicides}

Antifungals are commonly used as pesticides and pharmaceuticals and can pose a significant risk to terrestrial and aquatic organisms (Kookana et al. 1998; Komarek et al. 2010; Peng et al. 2012). Among the three antifungal chemicals tested in this study (Figure 5.4), ketoconazole, an antifungal agent used in human and veterinary pharmaceuticals (Zarn et al. 2003), was the most potent activator of both tunicate VDR/PXRa yeast bioassays generating $\mathrm{EC}_{50}$ values in the mid $\mathrm{nM}$ range (Table 5.1). Yeast strains expressing the $C$. intestinalis VDR/PXRa LBD were consistently activated by ketoconazole $\left(E_{50}=337-400\right.$ $\mu \mathrm{M}, \mathrm{CV}=5-18 \%$; Table 5.1). These results are comparable to in vivo studies showing acute toxicity of ketoconazole towards $D$. magna neonates $\left(E_{50}=2.8\right.$ $\mu \mathrm{M}$; Haeba et al. 2008). In contrast, the human PXR LBD was not activated by ketoconazole when tested in a transactivation assay using HepG2 cell lines (Sinz et al. 2006). This may be attributed to the fact that ketoconazole acts as a general inhibitor of human PXR by disrupting the interaction of human PXR with the co-activator, steroid receptor co-activator-1 (SRC-1; Huang et al. 2007). A SRC-1 orthologue could not be identified in the $C$. intestinalis genome sequence (A. Fidler, unpublished) which may allow activation of the tunicate VDR/PXRa LBD yeast bioassays by ketoconazole. These data suggest that the yeast bioassay may be used to detect pharmaceutical drugs that cannot be detected in mammalian cell lines expressing human PXR (Sinz et al. 2006). Detection of ketoconazole and other pharmaceutical compounds in the aquatic environment is of increasing importance because they can be relatively persistent leading to bioaccumulation in surface and waste waters (Kahle et al. 2008; Lindberg et al. 2010; Peng et al. 2012). Long-term consumption of these environmental pollutants can cause adverse health effects in most organisms, including humans (Oros et al. 2003). For example, ketoconazole has been implicated in affecting the endocrine systems of aquatic vertebrates since they interact with several cytochrome P-450 enzymes such as CYP19 which takes part in hormone communication pathways (Zarn et al. 2003; Hasselberg et al. 2008). 
The agricultural fungicide 2-phenylphenol was also active in yeast bioassays carrying the $C$. intestinalis and $B$. schlosseri VDR/PXRa LBD (pGAL4.CILBD.VP16, EC $\mathrm{E}_{50}=79 \mu \mathrm{M}$; pGAL4.BSLBD.VP16, $\mathrm{EC}_{50}=26 \mu \mathrm{M}$; Table 5.1). The responses were sigmoidal (Figure 5.4B) and relatively consistent (CV = 18-37\%; Table 5.1). Similarly, human PXR has been shown to be activated by 2-phenylphenol in a transactivation assay using COS-7 simian kidney cells which was slightly more sensitive than the yeast bioassay $\left(E_{20}=9.8 \mu \mathrm{M}\right.$; Kojima et al. 2011).

The fungal antibiotic radicicol, a potent and selective inhibitor of heat shock protein 90 (HSP90; Feldman et al. 2009), reliably induced $\beta$-galactosidase enzymatic activity in strains carrying plasmids pGAL4.CLBD $31 . \mathrm{VP} 16$, pGAL4.CILBD.VP16 and pGAL4.BSLBD.VP16 (CV = 9-17\%; Table 5.1, Figure $5.4 C$ ). Identification of radicicol as an activator of both tunicate VDR/PXRa LBDs tested $\left(E_{50}=9.5-13 \mu \mathrm{M}\right.$; Table 5.1) was consistent with its reported toxicity using tunicate larval metamorphosis bioassays (Ciona savignyi $\mathrm{IC}_{99}=$ $2.2 \mu \mathrm{M}$, Boltenia villosa $\mathrm{IC}_{99}=2.5 \mu \mathrm{M}$ ) suggesting that it may be toxic towards a wide range of chordates, including tunicates (Bishop et al. 2002; Cahill et al. 2012). These data indicate that the yeast bioassay may be a useful tool for monitoring these potent toxicants in the marine environment. 

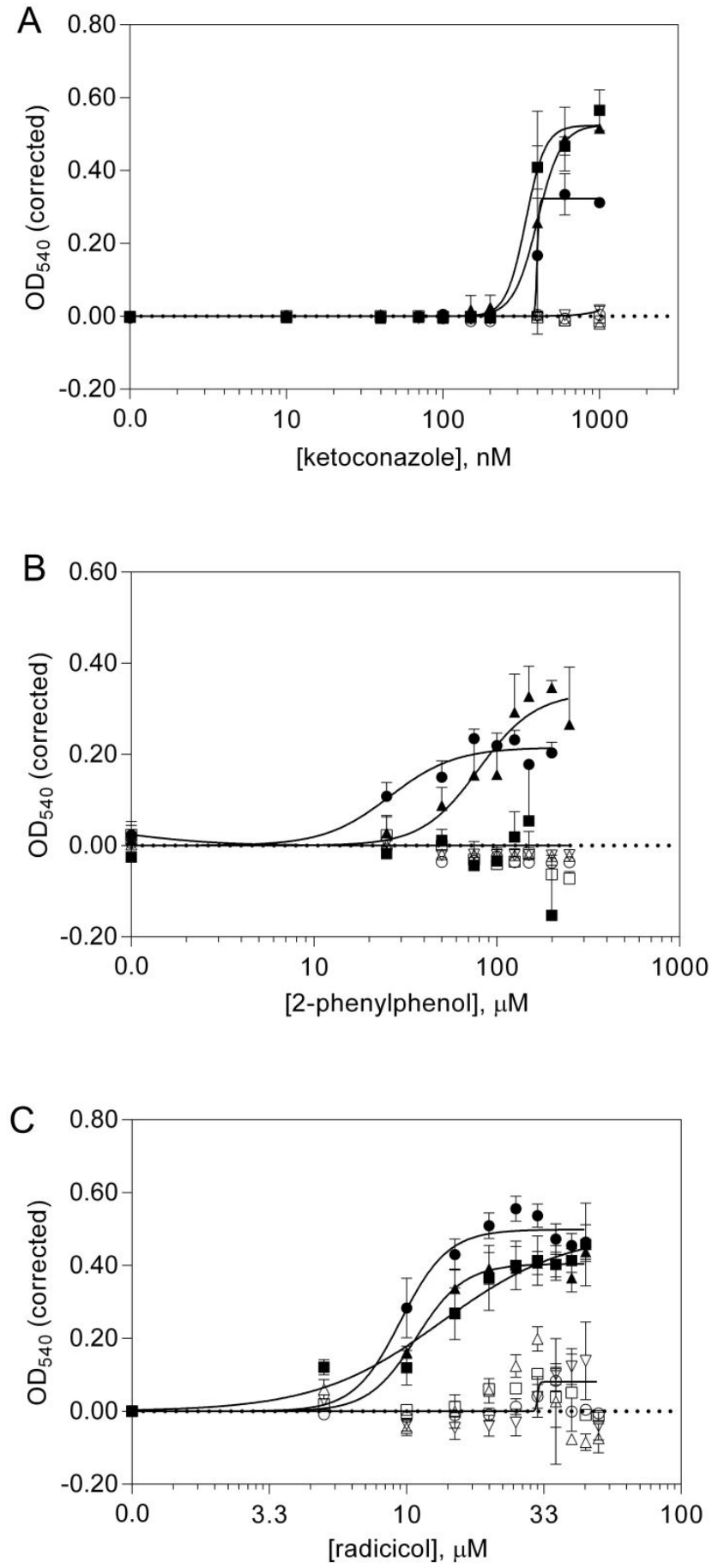

$\multimap$ pGAL4

$\neg$ pGAL4.VP16

$\triangle$ pGAL4.CiLBD

$\rightarrow$ pGAL4.BsLBD

$\rightarrow$ pGAL4.CiLBD $\Delta 31 . \mathrm{VP} 16$

$\leftarrow$ pGAL4.CiLBD.VP16

$\rightarrow$ pGAL4.BsLBD.VP16

Figure 5.4 Activities of fungicides tested in the yeast bioassays.

Responses of yeast strains carrying one of seven different pGBKT7-based expression vectors during exposure to a range of concentrations of: (A) ketoconazole, (B) 2phenylphenol and $(\mathbf{C})$ radicicol. $\beta$-galactosidase enzymatic activities, as measured by $\mathrm{OD}_{540}$ (corrected) values, were determined after $24 \mathrm{~h}$ or $48 \mathrm{~h}$ (Table 5.1). Data points represent means of $n=3$ replicates. Error bars show \pm one standard deviation. Dotted lines indicate baseline. 


\subsubsection{Activity of insecticide}

One of the most commonly used active ingredients in insect repellents, $n, n-$ diethyl- $m$-toluamide (DEET), was tested for its ability to activate the tunicate yeast bioassays. Yeast strains expressing the $C$. intestinalis VDR/PXR LBD (pGAL4.ClLBD 31 .VP16 and pGAL4.CILBD.VP16) showed concentrationdependent induction of $\beta$-galactosidase activity (Table 5.1, Figure 5.5A). In contrast, a negative effect on $\beta$-galactosidase induction was observed in strains expressing the $B$. schlosseri VDR/PXRa LBD (pGAL4.BsLBD.VP16; Figure $5.5 A)$. This suppression effect was also apparent in reduced $O_{620}$ values at high concentrations of DEET, suggesting a general toxicity towards this particular yeast strain (Appendix 17D). The $\mathrm{EC}_{50}$ values observed in this in vitro yeast bioassay (54-99 $\mu \mathrm{M}$; Table 5.1) were comparable to previously reported toxicity measures towards $D$. magna $\left(\mathrm{EC}_{50}=177-836 \mu \mathrm{M}\right.$; Seo et al. 2005; Weeks et al. 2012), the green alga Pseudokirchnierella subcapitata $\left(E_{50}=21\right.$ $\mu \mathrm{M}$; Harada et al. 2008) and the marine luminescent bacterium Photobacterium phosphoreum $\left(\mathrm{EC}_{50}=355 \mu \mathrm{M}\right.$; Kaiser \& Palabrica 1991) using in vivo acute toxicity bioassays. Although DEET is present in the aquatic environment worldwide (e.g. drinking water, streams, open seawater, groundwater and treated effluent), concentrations in surface waters are several hundreds of times lower than those toxic to aquatic organisms, and the probability for adverse effects to aquatic species is low (Costanzo et al. 2007; Weeks et al. 2012). However, identification of DEET as an activator of the $C$. intestinalis VDR/PXR LBD suggests that it may interact with invertebrate PXR orthologues. Due to the close phylogenetic relationship of tunicates and vertebrates (Delsuc et al. 2008), it could have adverse effects on vertebrate physiology via interaction with PXR. 


\subsubsection{Activity of pharmaceutical drugs}

Interest in the presence of pharmaceutical drugs in the environment has increased significantly over the last decade because hundreds of tonnes of these compounds are dispensed every year. For example, diclofenac sodium salt (diclofenac) is one of the most abundant pharmaceutically active compounds present in the water cycle (Heberer et al. 2002). Diclofenac induced $\beta$-galactosidase enzymatic activity in strains carrying plasmids pGAL4.ClBDA31.VP16, pGAL4.CLBD.VP16 and pGAL4.BsLBD.VP16 (Table 5.1 , Figure $5.5 \mathrm{~B})$ albeit with high variability $(\mathrm{CV}=38-44 \%$; Table 5.1$)$. The yeast strain expressing the $B$. schlosseri VDR/PXRa LBD, pGAL4.BsLBD.VP16, was more strongly activated by diclofenac than the strain expressing the $C$. intestinalis VDR/PXR $\alpha$ LBD, as indicated by the plateau levels of $\beta$ galactosidase production and $\mathrm{EC}_{50}$ values (Figure 5.5A, Table 5.1). The $\mathrm{EC}_{50}$ values observed in this study (11-91 $\mu \mathrm{M}$; Table 5.1) were in the same range as those previously reported for zebrafish (Danio rerio) using embryo toxicity tests $\left(E_{50}=17 \mu \mathrm{M}\right), D$. magna using acute immobilisation tests $\left(E_{50}=213 \mu \mathrm{M}\right)$ and planktonic green alga (Desmodesmus subspicatus) using growth inhibition tests $\left(E C_{50}=226 \mu \mathrm{M}\right.$; van den Brandhof \& Montforts 2010). Since these concentrations are classified as being potentially harmful to aquatic organisms (Cleuvers 2004), the yeast bioassay may provide an alternative method of detecting pharmaceuticals and harmful synthetic chemicals in the environment. 

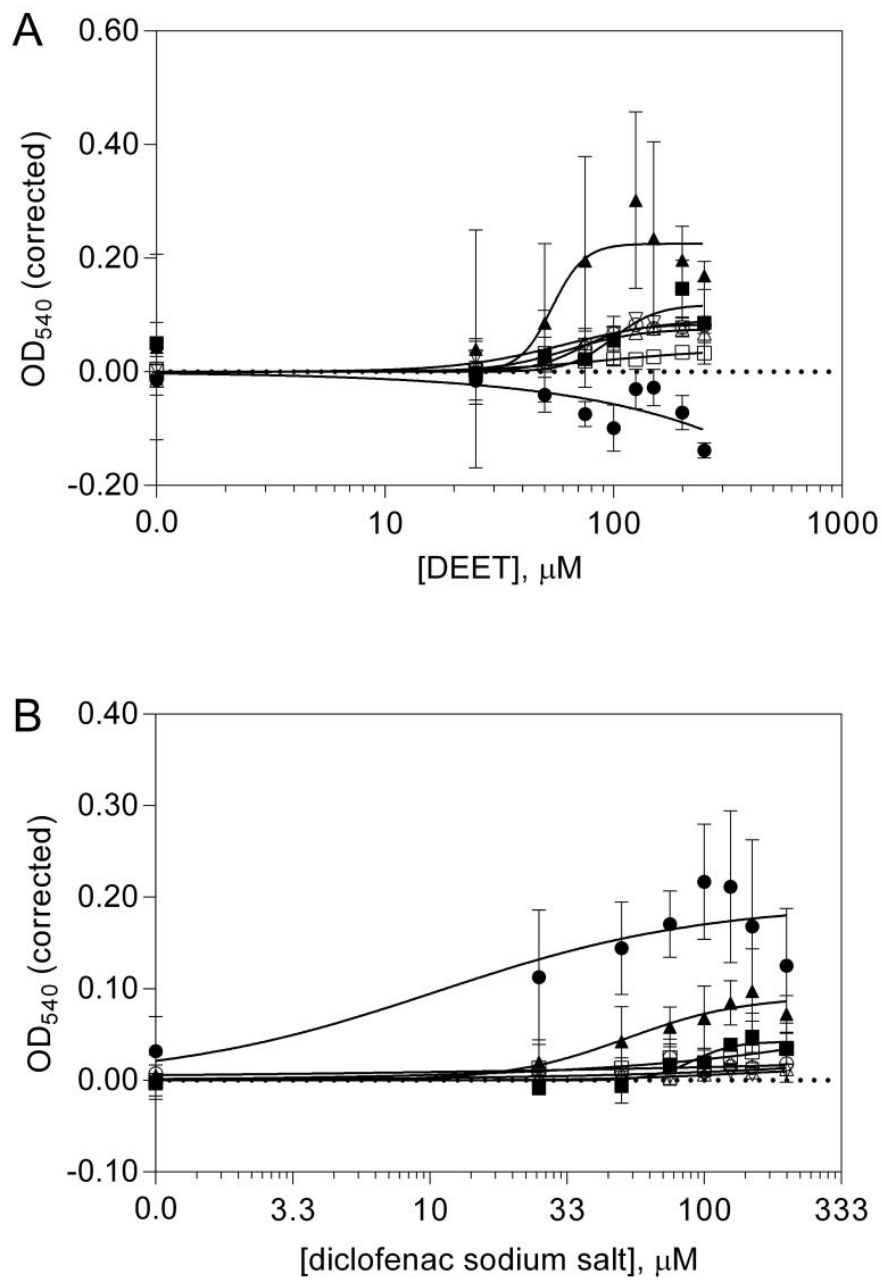

$$
\begin{aligned}
& \rightarrow \text { pGAL4 } \\
& \mp \text { pGAL4.VP16 } \\
& \star \text { pGAL4.CiLBD } \\
& \rightarrow \text { pGAL4.BSLBD } \\
& \leftarrow \text { pGAL4.CiLBD } 31 . V P 16 \\
& \leftarrow \text { pGAL4.CiLBD.VP16 } \\
& \rightarrow \text { pGAL4.BSLBD.VP16 }
\end{aligned}
$$

Figure 5.5 Activities of $n, n$-diethyl- $m$-toluamide (DEET) and diclofenac sodium salt tested in the yeast bioassays.

Responses of yeast strains carrying one of seven different pGBKT7-based expression vectors during exposure to a range of concentrations of $(\mathbf{A}) n, n$-diethyl- $m$-toluamide (DEET) and (B) diclofenac sodium salt. $\beta$-galactosidase enzymatic activities, as measured by $\mathrm{OD}_{540 \text { (corrected) }}$ values, were determined after $48 \mathrm{~h}$ (Table 5.1). Data points represent means of $n=3$ replicates. Error bars show \pm one standard deviation. Dotted lines indicate baseline. 


\subsubsection{Activity of commercial oil dispersants in the tunicate yeast bioassays}

Based on the findings that synthetic chemicals (e.g. pharmaceuticals, pesticides, etc.) can be detected using the yeast bioassay, five complex chemical mixtures that are frequently used to mitigate marine oil spills were analysed (Table 5.2). Three of the tested dispersants showed complete sigmoidal concentration-response curves in the tunicate yeast bioassays (Table 5.2, Figure 5.6A-C). Dispersant $D$ only activated the $C$. intestinalis VDR/PXRa LBD-based yeast bioassay but not the $B$. schlosseri VDR/PXRa equivalent (Figure 5.6D). Dispersant $E$ showed no ligand-dependent induction of $\beta$ galactosidase activity in any of the yeast strains tested (Figure 5.6E). The CVs of the $\mathrm{EC}_{50}$ values for oil dispersants that induced $\beta$-galactosidase activity in yeast strains were within the acceptable range of $6-19 \%$ (Table 5.2). These data $\left(E_{50}=67-207 \mathrm{mg} / \mathrm{L}\right)$ were comparable to previous in vivo studies using the marine rotifer Brachionus plicatilis ( $\mathrm{LC}_{50}=0.5-40 \mathrm{mg} / \mathrm{L}$; Kujawinski et al. 2011) indicating that oil dispersants are highly toxic towards micro-zooplankton. In addition, it has been shown that oil dispersants are also toxic towards a wide range of marine organisms including anemones, corals, crustaceans, starfish, molluscs, fish, and sperm whale (Wise \& Wise 2011; Goodbody-Gringley et al. 2013; Almeda et al. 2014). In addition, three commercially available dispersants have been shown to increase cytotoxicity in vitro $\left(\mathrm{LC}_{50}=250-400 \mathrm{mg} / \mathrm{L}\right)$ using HepG2 cells in a hepatotoxicity screening assay suggesting that dispersants can also cause adverse health effects in humans (Bandele et al. 2012; D'Andrea \& Reddy 2013). Further research is needed before clear conclusions can be drawn regarding the toxicity of dispersants towards marine organisms. However, efficient monitoring tools that allow early detection of these environmental contaminants are required to assist in mitigating the environmental impact and long-term consequences of these surfactants. 
Table 5.2 Activation of the tunicate yeast bioassays by five commercial oil dispersants.

Ligand-dependent induction of $\beta$-galactosidase enzymatic activity was measured in yeast strains carrying plasmids pGAL4.CILBD 431.VP16, pGAL4.CILBD.VP16 or pGAL4.BsLBD.VP16. Mean effective concentrations (EC 50 values) and 95\% confidence intervals $(95 \% \mathrm{Cl})$ are given in $\mathrm{mg} / \mathrm{L}$. 95\% confidence intervals represent variation within a triplicate measurement $(n=3)$. Coefficients of variance $(C V)$ are given in \% for triplicate intra-plate measurements. All oil dispersants were dissolved in ethanol.

\begin{tabular}{|c|c|c|c|c|c|c|c|c|}
\hline \multirow[t]{2}{*}{ Oil dispersant } & \multirow[t]{2}{*}{ Toxin type } & \multirow[t]{2}{*}{ Supplier } & \multicolumn{2}{|c|}{ pGAL4.CILBD $\triangle 31 . V P 16$} & \multicolumn{2}{|c|}{ pGAL4.CiLBD.VP16 } & \multicolumn{2}{|c|}{ pGAL4.BsLBD.VP16 } \\
\hline & & & $\mathrm{EC}_{50}(95 \% \mathrm{Cl})$ & CV & $\mathrm{EC}_{50}(95 \% \mathrm{Cl})$ & CV & $\mathrm{EC}_{50}(95 \% \mathrm{Cl})$ & CV \\
\hline Dispersant $A^{a}$ & Surfactant & Dr Tremblay & $101(88-117)$ & 11 & $67(53-83)$ & 10 & $118(21-660)$ & 6 \\
\hline Dispersant $\mathrm{B}^{\mathrm{a}}$ & Surfactant & Dr Tremblay & $163(138-193)$ & 13 & $151(120-191)$ & 16 & 169 (107-266l) & 6 \\
\hline Dispersant $\mathrm{C}^{\mathrm{a}}$ & Surfactant & Dr Tremblay & $137(105-179)$ & 11 & $126(104-152)$ & 19 & $207(161-266)$ & 12 \\
\hline Dispersant $\mathrm{D}^{\mathrm{a}}$ & Surfactant & Dr Tremblay & $92(63-135)$ & 14 & $146(123-174)$ & 10 & $\mathrm{NI}(\mathrm{NI})$ & DNC \\
\hline Dispersant $\mathrm{E}^{\mathrm{a}}$ & Surfactant & Dr Tremblay & $\mathrm{NI}(\mathrm{DNC})$ & DNC & $\mathrm{NI}(\mathrm{DNC})$ & DNC & $\sim 11$ (DNC) & 30 \\
\hline
\end{tabular}

Abbreviations: DNC, did not compute; NI, no induction of $\beta$-galactosidase enzymatic activity.

${ }^{\text {a }}$ Strains were incubated for 48 hours. 

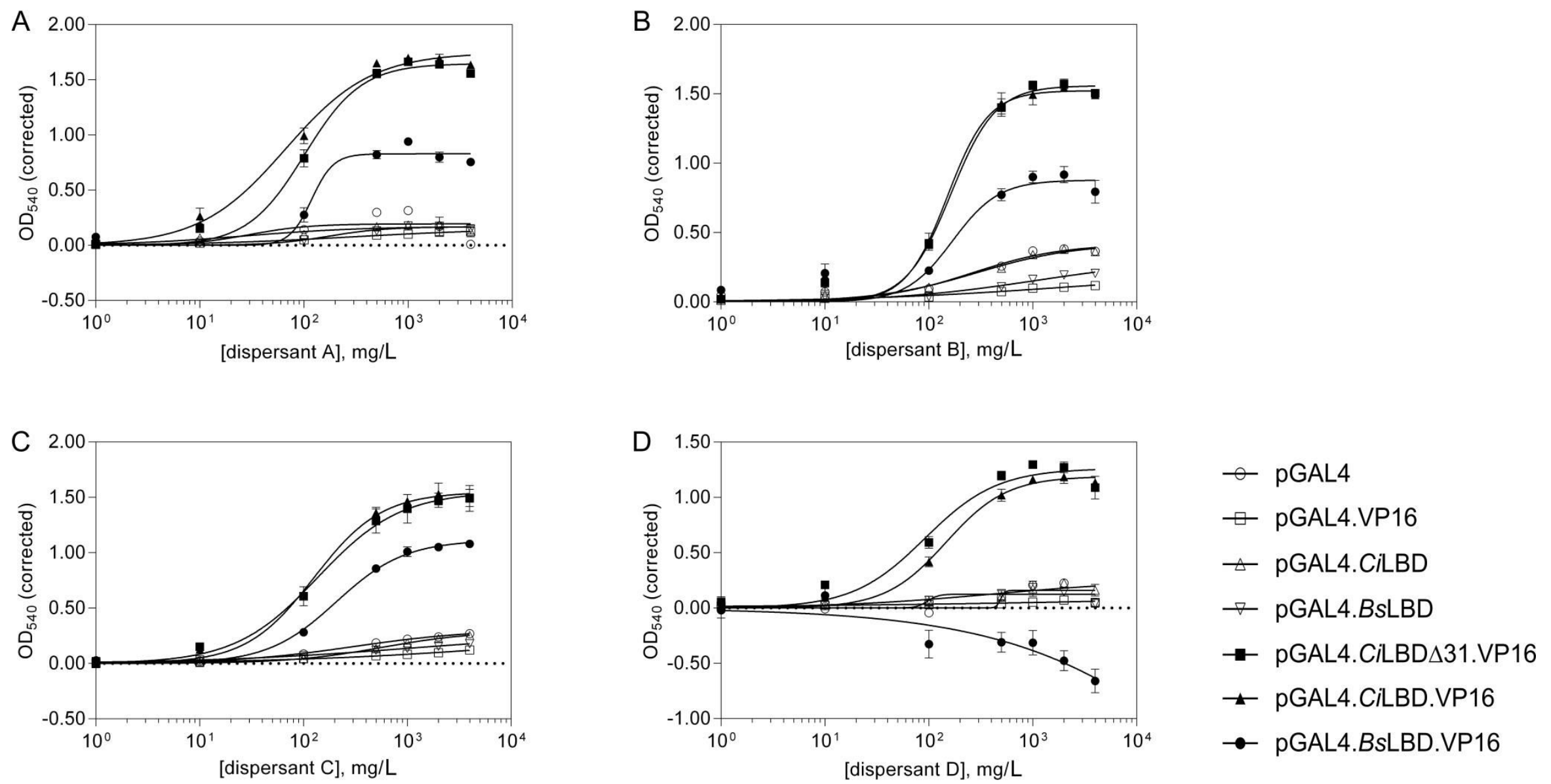

Figure 5.6 Activities of oil dispersants tested in the yeast bioassays.

Responses of yeast strains carrying one of seven different pGBKT7-based expression vectors during exposure to a range of concentrations of: (A) dispersant $A,(B)$ dispersant $B,(\mathbf{C})$ dispersant $\mathbf{C}$ and (D) dispersant $\mathrm{D}$. $\beta$-galactosidase enzymatic activities, as

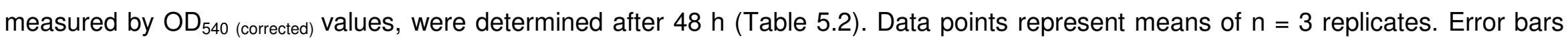
show \pm one standard deviation. Dotted lines indicate baseline. 


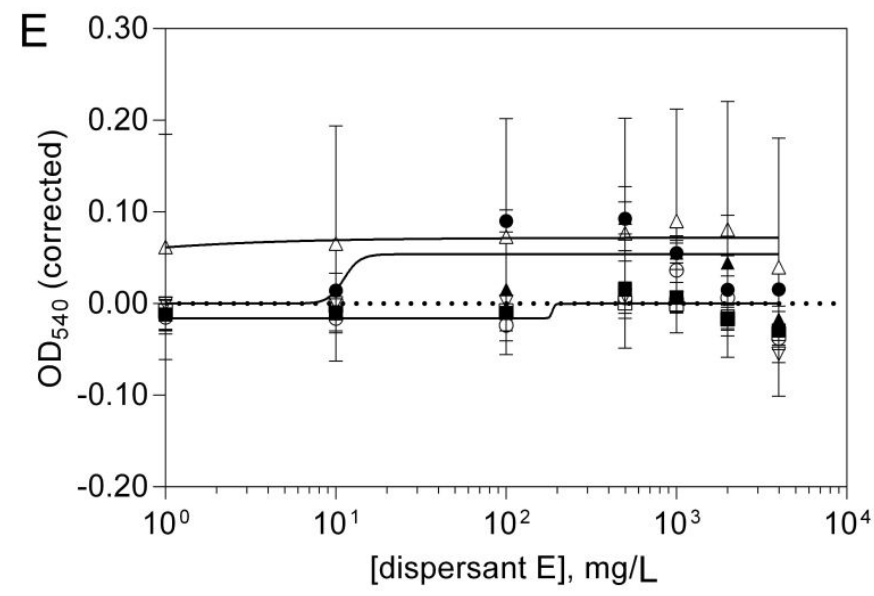

$\neg$ pGAL4

$\square$ pGAL4.VP16

$\triangle$ pGAL4.CiLBD

$\rightarrow$ pGAL4.BSLBD

$\rightarrow$ pGAL4.CiLBD $\triangle 31 . V P 16$

$\mp$ pGAL4.CiLBD.VP16

$\rightarrow$ pGAL4.BsLBD.VP16

Figure 5.6 (cont.) Activities of oil dispersants tested in the yeast bioassays.

Responses of yeast strains carrying one of seven different pGBKT7-based expression vectors during exposure to a range of concentrations of (E) dispersant E. $\beta$ galactosidase enzymatic activities, as measured by $\mathrm{OD}_{540}$ (corrected) values, were determined after $48 \mathrm{~h}$ (Table 5.2). Data points represent means of $\mathrm{n}=3$ replicates. Error bars show \pm one standard deviation. Dotted lines indicate baseline.

\subsubsection{Characteristics of the yeast bioassays}

Although the yeast bioassays were activated by a wide range of synthetic chemicals, there was considerable variation of activity between different test compounds (Table 5.1). This may be due to structural differences between the chemicals analysed, which are likely to affect the receptor-binding affinity. As has been previously reported for a $C$. intestinalis VDR/PXRa LBD-based mammalian cell line assay (Fidler et al. 2012; Chapter Three), the tunicate VDR/PXRa LBD-based yeast bioassay appeared to be more sensitive towards structurally complex chemicals (e.g. ketoconazole, radicicol; Figure 5.1l\&K and 5.4A\&C) Among the remaining compounds, a clear correlation between assay sensitivity and compound structure could not be identified. For example, yeast bioassays were highly sensitive towards butylated hydroxytoluene but were less sensitive towards the structurally similar 2-phenoxyethanol (Figure 5.1A\&C and 5.2A\&C, Table 5.1). Another possible explanation for the high assay variability is the solubility of the test compounds in ethanol. 2-phenylphenol, 4-chloro-3,5- 
dimethylphenol and triclosan are practically insoluble in ethanol. Consequently, follow-up studies were undertaken where all 13 synthetic chemicals were dissolved in DMSO and retested in the yeast bioassays. Compounds that were dissolved in DMSO generally showed reduced sensitivity (higher $\mathrm{EC}_{50}$ values) but with increased variability (greater $\mathrm{CV}$ ) when compared to data generated from compounds dissolved in ethanol (Appendix 16). It also appeared that the high assay variability was more evident with the low level $\beta$-galactosidase enzymatic activities (corrected $\mathrm{OD}_{540}<0.2$ ) as indicated by shallow log-dose response curves (Table 5.1, Figures 5.2-5.5).

To overcome these differences in sensitivity, additional organic solvents (e.g. acetone) should be considered in future bioassays as it is likely to result in improved assay sensitivity and consistency for certain compounds. In addition, an increase in replicate numbers (e.g. five replicates) and robotic liquid handling may improve reliability and repeatability of the bioassay as high intra-assay variability often reflects issues arising from pipetting very small volumes (Schultheiss \& Stanton 2009).

The data from this chapter shows that tunicate yeast bioassays represent an accurate, sensitive and reliable alternative to traditional in vivo toxicity screening assays for some of the compounds tested (e.g. butylated hydroxytoluene, ketoconazole and radicicol). Based on biologically relevant receptors, the tunicate VDR/PXRa LBD-based yeast bioassay may provide consistent proxies for assessing the effects of environmental contaminants on invertebrate members of coastal ecosystems (Stewart et al. 2014).

One of the main advantages of the yeast bioassay over in vivo assays is the ability to assess the toxicity of complex mixtures rather than single, wellcharacterised pure compounds. Analysis of complex mixtures is particularly important when assessing toxicity of environmental pollutants because the additive interaction of these compounds in mixtures can cause different biological effects compared to pure compounds (Kinani et al. 2010; Bandele et al. 2012). Additive mixture effects have been demonstrated in vitro for antiandrogenic pesticides, for compounds with estrogenic properties, and for compounds tested in vivo (Payne et al. 2000; Birkhoj et al. 2004). Low exposure to multiple EDCs with similar modes of action has also been shown to deliver a 
toxicological response, even though the effect of the individual compounds was below the limit of detection (Silva et al. 2002; Christiansen et al. 2008). The tunicate yeast bioassay may provide an alternative method to fill this gap.

\subsection{Conclusion}

The tunicate VDR/PXRa LBD-based yeast bioassays tested in this study were activated by a wide range of structurally diverse synthetic chemicals. These chemicals included bioactive compounds frequently used as preservatives in; foods, drugs and cosmetics, as UV-filters in personal care products, and as antifungal agents in pesticides and pharmaceuticals. This is of particular importance because many of these chemicals are frequently discharged into the environment, and can disrupt normal physiological functions (Oros et al. 2003; Kinani et al. 2010; Stewart et al. 2014).

This chapter highlights the feasibility of using tunicate VDR/PXRa LBD-based yeast bioassays for detecting anthropogenic chemicals. Future research should focus on testing mixtures, formulations and environmental samples in the yeast bioassays. Combining these in vitro yeast bioassays with detailed chemical analyses will provide a powerful tool to detect and identify bioactive compounds within complex samples (Eggen \& Segner 2003). An important aspect to be considered when developing the tunicate yeast bioassay into an environmental monitoring tool is the extreme permissiveness of the tunicate VDR/PXR LBD towards a wide range of ligands (Watkins et al. 2001; Watkins et al. 2003; Chrencik et al. 2005). It is likely that the yeast bioassay is less sensitive towards certain classes of pollutants (e.g. EDCs) when compared to other NR-reporter assays (e.g. ERa reporter assay), which use highly selective NR LBDs that bind a structurally limited range of ligands (Routledge \& Sumpter 1996; Zhang et al. 2004; Krasowski et al. 2005). However, the ability to detect a wide range of structurally diverse bioactive chemicals makes the tunicate yeast bioassay a valuable tool for high-throughput screening of environmental samples. 


\subsection{References}

Agatonovic-Kustrin S., Morton D.W., Razic S. 2014. In silico modelling of pesticide aquatic toxicity. Combinatorial Chemistry and High Throughput Screening 17(9): 808-18. doi: 10.2174/1386207317666141021110738

Almeda R., Hyatt C., Buskey E.J. 2014. Toxicity of dispersant Corexit 9500A and crude oil to marine microzooplankton. Ecotoxicology and Environmental Safety 106: 76-85. doi: 10.1016/j.ecoenv.2014.04.028

Ball A., Truskewycz A. 2013. Polyaromatic hydrocarbon exposure: an ecological impact ambiguity. Environmental Science and Pollution Research International 20(7): 4311-26. doi: 10.1007/s11356-013-1620-2

Bandele O.J., Santillo M.F., Ferguson M., Wiesenfeld P.L. 2012. In vitro toxicity screening of chemical mixtures using HepG2/C3A cells. Food and Chemical Toxicology 50(5): 1653-9. doi: 10.1016/j.fct.2012.02.016

Birkhoj M., Nellemann C., Jarfelt K., Jacobsen H., Andersen H.R., Dalgaard M., Vinggaard A.M. 2004. The combined antiandrogenic effects of five commonly used pesticides. Toxicology and Applied Pharmacology 201(1): 10-20. doi:10.1016/j.taap.2004.04.016

Bis R.L., Mallela K.M. 2014. Antimicrobial preservatives induce aggregation of interferon alpha-2a: the order in which preservatives induce protein aggregation is independent of the protein. International Journal of Pharmaceutics 472(1-2): 356-61. doi: 10.1016/j.ijpharm.2014.06.044

Bishop C.D., Bates W.R., Brandhorst B.P. 2002. HSP90 function is required for morphogenesis in ascidian and echinoid embryos. Development Genes and Evolution 212(2): 70-80. doi: 10.1007/s00427-002-0212-9

Bovee T.F.H., Helsdingen R.J.R., Koks P.D., Kuiper H.A., Hoogenboom R.L.A.P., Keijer J. 2004. Development of a rapid yeast estrogen bioassay, based on the expression of green fluorescent protein. Gene 325(0): 187-200. doi: 10.1016/j.gene.2003.10.015

Cahill P., Heasman K., Jeffs A., Kuhajek J., Mountfort D. 2012. Preventing ascidian fouling in aquaculture: screening selected allelochemicals for anti-metamorphic properties in ascidian larvae. Biofouling 28(1): 39-49. doi: 10.1080/08927014.2011.648624

Chrencik J.E., Orans J., Moore L.B., Xue Y., Peng L., Collins J.L., Wisely G.B., Lambert M.H., Kliewer S.A., Redinbo M.R. 2005. Structural disorder in the complex of human pregnane $X$ receptor and the macrolide antibiotic rifampicin. Molecular Endocrinology 19(5): 1125-34. doi: 10.1210/me.2004-0346

Christiansen S., Scholze M., Axelstad M., Boberg J., Kortenkamp A., Hass U. 2008. Combined exposure to anti-androgens causes markedly increased frequencies of hypospadias in the rat. International Journal of Andrology 31(2): 241-8. doi: 10.1111/j.1365-2605.2008.00866.x.

Cleuvers M. 2004. Mixture toxicity of the anti-inflammatory drugs diclofenac, ibuprofen, naproxen and acetylsalicylic acid. Ecotoxicology and Environmental Safety 59(3): 309-15. doi: 10.1016/s0147-6513(03)001416

Colosi J.C., Kney A.D. 2011. A yeast estrogen screen without extraction provides fast, reliable measures of estrogenic activity. Environmental Toxicology and Chemistry 30(10): 2261-9. doi: 10.1002/etc.618 
Costanzo S.D., Watkinson A.J., Murby E.J., Kolpin D.W., Sandstrom M.W. 2007. Is there a risk associated with the insect repellent DEET ( $n, n-$ diethyl- $m$-toluamide) commonly found in aquatic environments? Science of the Total Environment 384(1-3): 214-20. doi:

10.1016/j.scitotenv.2007.05.036

Creusot N., Budzinski H., Balaguer P., Kinani S., Porcher J.-M., Ait-Aissa S. 2013. Effect-directed analysis of endocrine-disrupting compounds in multi-contaminated sediment: identification of novel ligands of estrogen and pregnane $\mathrm{X}$ receptors. Analytical and Bioanalytical Chemistry 405(8): 2553-66. doi: 10.1007/s00216-013-6708-5

Creusot N., Kinani S., Balaguer P., Tapie N., LeMenach K., Maillot-Marechal E., Porcher J.-M., Budzinski H., Ait-Aissa S. 2010. Evaluation of an hPXR reporter gene assay for the detection of aquatic emerging pollutants: screening of chemicals and application to water samples. Analytical and Bioanalytical Chemistry 396(2): 569-83. doi: 10.1007/s00216-009-3310-y

D'Andrea M.A., Reddy G.K. 2013. Health consequences among subjects involved in gulf oil spill clean-up activities. American Journal of Medicine 126(11): 966-74. doi: 10.1016/j.amjmed.2013.05.014

Daughton C.G. 2002. Environmental stewardship and drugs as pollutants. Lancet 360(9339): 1035-6. doi: 10.1016/s0140-6736(02)11176-7

Daughton C.G., Ternes T.A. 1999. Pharmaceuticals and personal care products in the environment: agents of subtle change? Environmental Health Perspectives 107: 907-38. doi: 10.2307/3434573

De Coster S., van Larebeke N. 2012. Endocrine-disrupting chemicals: associated disorders and mechanisms of action. Journal of Environmental and Public Health 2012: 52. doi: 10.1155/2012/713696

Delsuc F., Tsagkogeorga G., Lartillot N., Philippe H. 2008. Additional molecular support for the new chordate phylogeny. Genesis 46(11): 592-604. doi: $10.1002 / \mathrm{dvg} .20450$

Dybdahl M., Nikolov N.G., Wedebye E.B., Jonsdottir S.O., Niemela J.R. 2012. QSAR model for human pregnane $X$ receptor (PXR) binding: screening of environmental chemicals and correlations with genotoxicity, endocrine disruption and teratogenicity. Toxicology and Applied Pharmacology 262(3): 301-9. doi: 10.1016/j.taap.2012.05.008

Eggen R.I., Segner H. 2003. The potential of mechanism-based bioanalytical tools in ecotoxicological exposure and effect assessment. Analytical and Bioanalytical Chemistry 377(3): 386-96. doi: 10.1007/s00216-003-2059-y

Ekins S., Reschly E.J., Hagey L.R., Krasowski M.D. 2008. Evolution of pharmacologic specificity in the pregnane $\mathrm{X}$ receptor. BMC Evolutionary Biology 8. doi: 10.1186/1471-2148-8-103

European Commission. 2008. Priority substances and certain other pollutants according to Annex II of Directive 2008/105/EC.

Feldman R.I., Mintzer B., Zhu D., Wu J.M., Biroc S.L., Yuan S., Emayan K., Chang Z., Chen D., Arnaiz D.O.et al. 2009. Potent triazolothione inhibitor of heat-shock protein-90. Chemical Biology and Drug Design 74(1): 4350. doi: 10.1111/j.1747-0285.2009.00833.x.

Fent K., Weston A.A., Caminada D. 2006. Ecotoxicology of human pharmaceuticals. Aquatic Toxicology 76(2): 122-59. doi: 10.1016/j.aquatox.2005.09.009

Fent K., Kunz P.Y., Zenker A., Rapp M. 2010. A tentative environmental risk assessment of the UV-filters 3-(4-methylbenzylidene-camphor), 2-ethyl- 
hexyl-4-trimethoxycinnamate, benzophenone-3, benzophenone-4 and 3benzylidene camphor. Marine Environmental Research 69, Supplement 1(0): S4-S6. doi: 10.1016/j.marenvres.2009.10.010

Fidler A.E., Holland P.T., Reschly E.J., Ekins S., Krasowski M.D. 2012. Activation of a tunicate (Ciona intestinalis) xenobiotic receptor orthologue by both natural toxins and synthetic toxicants. Toxicon 59: 365-72. doi: 10.1016/j.toxicon.2011.12.008

Fiocco R.J., Lewis A. 1999. Oil spill dispersants. Pure and Applied Chemistry 71(1): 27-42. doi: 10.1351/pac199971010027

Fuller C., Bonner J., Page C., Ernest A., McDonald T., McDonald S. 2004. Comparative toxicity of oil, dispersant and oil plus dispersant to several marine species. Environmental Toxicology and Chemistry 23(12): 29419. doi: 10.1897/03-548.1

Gavrilescu M., Demnerova K., Aamand J., Agathos S., Fava F. 2015. Emerging pollutants in the environment: present and future challenges in biomonitoring, ecological risks and bioremediation. New Biotechnology 32(1): 147-56. doi: 10.1016/j.nbt.2014.01.001

Golbamaki A., Cassano A., Lombardo A., Moggio Y., Colafranceschi M., Benfenati E. 2014. Comparison of in silico models for prediction of Daphnia magna acute toxicity. SAR QSAR Environmental Research 25(8): 673-94. doi: 10.1080/1062936X.2014.923041

Gomez E., Pillon A., Fenet H., Rosain D., Duchesne M.J., Nicolas J.C., Balaguer P., Casellas C. 2005. Estrogenic activity of cosmetic components in reporter cell lines: parabens, UV screens and musks. Journal of Toxicology and Environmental Health 68(4): 239-51. doi: 10.1080/15287390590895054

Goodbody-Gringley G., Wetzel D.L., Gillon D., Pulster E., Miller A., Ritchie K.B. 2013. Toxicity of Deepwater Horizon source oil and the chemical dispersant, Corexit (R) 9500, to coral larvae. PloS One 8(1). doi: 10.1371/journal.pone.0045574

Grun F., Blumberg B. 2009. Endocrine disrupters as obesogens. Molecular and Cellular Endocrinology 304(1-2): 19-29. doi: 10.1016/j.mce.2009.02.018

Haeba M.H., Hilscherova K., Mazurova E., Blaha L. 2008. Selected endocrine disrupting compounds (vinclozolin, flutamide, ketoconazole and dicofol): effects on survival, occurrence of males, growth, molting, and reproduction of Daphnia magna. Environmental Science and Pollution Research International 15(3): 222-7.

Harada A., Komori K., Nakada N., Kitamura K., Suzuki Y. 2008. Biological effects of PPCPs on aquatic lives and evaluation of river waters affected by different wastewater treatment levels. Water Science and Technology 58(8): 1541-6. doi: 10.2166/wst.2008.742

Hasselberg L., Westerberg S., Wassmur B., Celander M.C. 2008.

Ketoconazole, an antifungal imidazole, increases the sensitivity of rainbow trout to 17alpha-ethynylestradiol exposure. Aquatic Toxicology 86(2): 256-64. doi: 10.1016/j.aquatox.2007.11.006

Heberer T., Reddersen K., Mechlinski A. 2002. From municipal sewage to drinking water: fate and removal of pharmaceutical residues in the aquatic environment in urban areas. Water Science and Technology 46(3): 81-8.

Hill E.M., Evans K.L., Horwood J., Rostkowski P., Oladapo F.O., Gibson R., Shears J.A., Tyler C.R. 2010. Profiles and some initial identifications of 
(anti)androgenic compounds in fish exposed to wastewater treatment works effluents. Environmental Science and Technology 44(3): 1137-43. doi: $10.1021 /$ es $901837 n$

Houtman C.J., van Oostveen A.M., Brouwer A., Lamoree M.H., Legler J. 2004a. Identification of estrogenic compounds in fish bile using bioassaydirected fractionation. Environmental Science and Technology 38(23): 6415-23. doi: 10.1021/es049750p

Houtman C.J., Cenijn P.H., Hamers T., Lamoree M.H., Legler J., Murk A.J., Brouwer A. 2004b. Toxicological profiling of sediments using in vitro bioassays, with emphasis on endocrine disruption. Environmental Toxicology and Chemistry 23(1): 32-40. doi: 10.1897/02-544

Huang H., Wang H., Sinz M., Zoeckler M., Staudinger J., Redinbo M.R., Teotico D.G., Locker J., Kalpana G.V., Mani S. 2007. Inhibition of drug metabolism by blocking the activation of nuclear receptors by ketoconazole. Oncogene 26(2): 258-68. doi: 10.1038/sj.onc.1209788

Jacobs M.N., Nolan G.T., Hood S.R. 2005. Lignans, bacteriocides and organochlorine compounds activate the human pregnane $\mathrm{X}$ receptor (PXR). Toxicology and Applied Pharmacology 209(2): 123-33. doi: 10.1016/j.taap.2005.03.015

Järup L. 2003. Hazards of heavy metal contamination. British Medical Bulletin 68(1): 167-82. doi: 10.1093/bmb/ldg032

Kahle M., Buerge I.J., Hauser A., Muller M.D., Poiger T. 2008. Azole fungicides: occurrence and fate in wastewater and surface waters. Environmental Science and Technology 42(19): 7193-200. doi: 10.1021/es8009309

Kaiser K.L.E., Palabrica V.S. 1991. Photobacterium phosphoreum toxicity data index. Water Pollution Research Journal of Canada 26(3): 361-431.

Karami-Mohajeri S., Abdollahi M. 2011. Toxic influence of organophosphate, carbamate and organochlorine pesticides on cellular metabolism of lipids, proteins and carbohydrates: a systematic review. Human and Experimental Toxicology 30(9): 1119-40. doi:

$10.1177 / 0960327110388959$

Kasprzyk-Hordern B., Dinsdale R.M., Guwy A.J. 2008. The occurrence of pharmaceuticals, personal care products, endocrine disruptors and illicit drugs in surface water in South Wales, UK. Water Research 42(13): 3498-518. doi: 10.1016/j.watres.2008.04.026

Kimura K., Kameda Y., Yamamoto H., Nakada N., Tamura I., Miyazaki M., Masunaga S. 2014. Occurrence of preservatives and antimicrobials in Japanese rivers. Chemosphere 107: 393-9. doi:

10.1016/j.chemosphere.2014.01.008

Kinani S., Bouchonnet S., Creusot N., Bourcier S., Balaguer P., Porcher J.-M., Ait-Aissa S. 2010. Bioanalytical characterisation of multiple endocrineand dioxin-like activities in sediments from reference and impacted small rivers. Environmental Pollution 158(1): 74-83. doi:

10.1016/j.envpol.2009.07.041

Kojima H., Takeuchi S., Nagai T. 2010. Endocrine-disrupting potential of pesticides via nuclear receptors and aryl hydrocarbon receptor. Journal of Health Science 56(4): 374-86. doi: 10.1016/j.reprotox.2010.07.009

Kojima H., Sata F., Takeuchi S., Sueyoshi T., Nagai T. 2011. Comparative study of human and mouse pregnane $X$ receptor agonistic activity in 200 pesticides using in vitro reporter gene assays. Toxicology 280(3): 77-87. doi: $10.1016 /$ j.tox.2010.11.008 
Kolpin D.W., Skopec M., Meyer M.T., Furlong E.T., Zaugg S.D. 2004. Urban contribution of pharmaceuticals and other organic wastewater contaminants to streams during differing flow conditions. Science of the Total Environment 328(1-3): 119-30. doi:

10.1016/j.scitotenv.2004.01.015

Kolpin D.W., Furlong E.T., Meyer M.T., Thurman E.M., Zaugg S.D., Barber L.B., Buxton H.T. 2002. Pharmaceuticals, hormones and other organic wastewater contaminants in U.S. streams, 1999-2000: a national reconnaissance. Environmental Science and Technology 36(6): 1202-11. doi: $10.1021 /$ es011055j

Komarek M., Cadkova E., Chrastny V., Bordas F., Bollinger J.C. 2010. Contamination of vineyard soils with fungicides: a review of environmental and toxicological aspects. Environment International 36(1): 138-51. doi: 10.1016/j.envint.2009.10.005

Kookana R.S., Baskaran S., Naidu R. 1998. Pesticide fate and behaviour in Australian soils in relation to contamination and management of soil and water: a review. Australian Journal of Soil Research 36(5): 715-64. doi: 10.1071/s97109

Krasowski M.D., Yasuda K., Hagey L.R., Schuetz E.G. 2005. Evolutionary selection across the nuclear hormone receptor superfamily with a focus on the NR1I subfamily (vitamin D, pregnane $X$ and constitutive androstane receptors). Nuclear Receptor 3: 2. doi: 10.1186/1478-1336-32

Kujawinski E.B., Soule M.C.K., Valentine D.L., Boysen A.K., Longnecker K., Redmond M.C. 2011. Fate of dispersants associated with the Deepwater Horizon oil spill. Environmental Science and Technology 45(4): 1298306. doi: 10.1021/es103838p

Kunz P.Y., Fent K. 2006. Multiple hormonal activities of UV-filters and comparison of in vivo and in vitro estrogenic activity of ethyl-4aminobenzoate in fish. Aquatic Toxicology 79(4): 305-24. doi: 10.1016/j.aquatox.2006.06.016

Langford K.H., Thomas K.V. 2008. Inputs of chemicals from recreational activities into the Norwegian coastal zone. Journal of Environmental Monitoring 10(7): 894-8. doi: 10.1039/B806198J

Lemaire G., Mnif W., Pascussi J.M., Pillon A., Rabenoelina F., Fenet H., Gomez E., Casellas C., Nicolas J.C., Cavailles V.et al. 2006. Identification of new human pregnane $X$ receptor ligands among pesticides using a stable reporter cell system. Toxicological Sciences 91(2): 501-9. doi: 10.1093/toxsci/kfj173

Lindberg R.H., Fick J., Tysklind M. 2010. Screening of antimycotics in Swedish sewage treatment plants- waters and sludge. Water Research 44(2): 649-57. doi: 10.1016/j.watres.2009.10.034

Lindstrom A., Buerge I.J., Poiger T., Bergqvist P.A., Muller M.D., Buser H.R. 2002. Occurrence and environmental behavior of the bactericide triclosan and its methyl derivative in surface waters and in wastewater. Environmental Science and Technology 36(11): 2322-9. doi: 10.1021/es0114254

Meyer B.K., Ni A., Hu B., Shi L. 2007. Antimicrobial preservative use in parenteral products: past and present. Journal of Pharmaceutical Sciences 96(12): 3155-67. doi: 10.1002/jps.20976 
Mikamo E., Harada S., Nishikawa J., Nishihara T. 2003. Endocrine disruptors induce cytochrome $\mathrm{P} 450$ by affecting transcriptional regulation via pregnane X receptor. Toxicology and Applied Pharmacology 193(1): 6672. doi: 10.1016/j.taap.2003.08.001

Miller D., Wheals B.B., Beresford N., Sumpter J.P. 2001. Estrogenic activity of phenolic additives determined by an in vitro yeast bioassay. Environmental Health Perspectives 109(2): 133-8.

Mnif W., Hassine A.I.H., Zidi I., Dagnino S., Bouaziz A., Fenet H., Hamouda Y.H., Balaguer P., Bartegi A. 2011. Occurrence of estrogenic and pregnane $\mathrm{X}$ receptor specific activities in Tunisian sewage treatment plants using a panel of bioassays. Immuno-Analyse and Biologie Specialisee 26(1): 1-5. doi: 10.1016/j.immbio.2010.10.003

Odermatt A., Gumy C., Atanasov A.G., Dzyakanchuk A.A. 2006. Disruption of glucocorticoid action by environmental chemicals: potential mechanisms and relevance. Journal of Steroid Biochemistry and Molecular Biology 102(1-5): 222-31. doi:10.1016/j.jsbmb.2006.09.010

Oros D.R., Jarman W.M., Lowe T., David N., Lowe S., Davis J.A. 2003. Surveillance for previously unmonitored organic contaminants in the San Francisco Estuary. Marine Pollution Bulletin 46(9): 1102-10. doi: 10.1016/s0025-326x(03)00248-0

Payne J., Rajapakse N., Wilkins M., Kortenkamp A. 2000. Prediction and assessment of the effects of mixtures of four xenoestrogens. Environmental Health Perspectives 108(10): 983-7. doi: 10.1289/ehp.00108983

Peng X., Huang Q., Zhang K., Yu Y., Wang Z., Wang C. 2012. Distribution, behavior and fate of azole antifungals during mechanical, biological and chemical treatments in sewage treatment plants in China. Science of the Total Environment 426: 311-7. doi: 10.1016/j.scitotenv.2012.03.067

Routledge E.J., Sumpter J.P. 1996. Estrogenic activity of surfactants and some of their degradation products assessed using a recombinant yeast screen. Environmental Toxicology and Chemistry 15(3): 241-8. doi: 10.1002/etc.5620150303

Routledge E.J., Parker J., Odum J., Ashby J., Sumpter J.P. 1998. Some alkyl hydroxy benzoate preservatives (parabens) are estrogenic. Toxicology and Applied Pharmacology 153(1): 12-9. doi: 10.1006/taap.1998.8544

Schultheiss O.C., Stanton S.J. 2009. Assessment of salivary hormones. In: Harmon-Jones E, Beer JS ed. Methods in Social Neuroscience. New York, Guilford Press.

Seo J., Lee Y.G., Kim S.D., Cha C.J., Ahn J.H., Hur H.G. 2005. Biodegradation of the insecticide $n, n$-diethyl- $m$-toluamide by fungi: identification and toxicity of metabolites. Archives of Environmental Contamination and Toxicology 48(3): 323-8. doi: 10.1007/s00244-004-0029-9

Silva E., Rajapakse N., Kortenkamp A. 2002. Something from "nothing"- eight weak estrogenic chemicals combined at concentrations below NOECs produce significant mixture effects. Environmental Science and Technology 36(8): 1751-6. doi: 10.1021/es0101227

Singh K.P., Gupta S., Kumar A., Mohan D. 2014. Multispecies QSAR modeling for predicting the aquatic toxicity of diverse organic chemicals for regulatory toxicology. Chemical Research in Toxicology 27(5): 741-53. doi: $10.1021 / \mathrm{t} \times 400371 \mathrm{w}$ 
Sinz M., Kim S., Zhu Z., Chen T., Anthony M., Dickinson K., Rodrigues A.D. 2006. Evaluation of 170 xenobiotics as transactivators of human pregnane $\mathrm{X}$ receptor (hPXR) and correlation to known CYP3A4 drug interactions. Current Drug Metabolism 7(4): 375-88. doi: $10.2174 / 138920006776873535$

Soni M.G., Burdock G.A., Taylor S.L., Greenberg N.A. 2001. Safety assessment of propyl paraben: a review of the published literature. Food and Chemical Toxicology 39(6): 513-32. doi: 10.1016/S0278-6915(00)001629

Stewart M., Olsen G., Hickey C.W., Ferreira B., Jelic A., Petrovic M., Barcelo D. 2014. A survey of emerging contaminants in the estuarine receiving environment around Auckland, New Zealand. Science of the Total Environment 468: 202-10. doi: 10.1016/j.scitotenv.2013.08.039

Terasaki M., Makino M., Tatarazako N. 2009. Acute toxicity of parabens and their chlorinated by-products with Daphnia magna and Vibrio fischeri bioassays. Journal of Applied Toxicology 29(3): 242-7. doi: 10.1002/jat.1402

Thomas K.V., Hurst M.R., Matthiessen P., McHugh M., Smith A., Waldock M.J. 2002. An assessment of in vitro androgenic activity and the identification of environmental androgens in United Kingdom estuaries. Environmental Toxicology and Chemistry 21(7): 1456-61. doi: 10.1002/etc.5620210717

Timsit Y.E., Negishi M. 2007. CAR and PXR: the xenobiotic-sensing receptors. Steroids 72(3): 231-46. doi: 10.1016/j.steroids.2006.12.006

van den Brandhof E.-J., Montforts M. 2010. Fish embryo toxicity of carbamazepine, diclofenac and metoprolol. Ecotoxicology and Environmental Safety 73(8): 1862-6. doi: 10.1016/j.ecoenv.2010.08.031

Vasquez M.I., Lambrianides A., Schneider M., Kuemmerer K., Fatta-Kassinos D. 2014. Environmental side effects of pharmaceutical cocktails: what we know and what we should know. Journal of Hazardous Materials 279: 169-89. doi: 10.1016/j.jhazmat.2014.06.069

Vedani A., Smiesko M., Spreafico M., Peristera O., Dobler M. 2009. VirtualToxLab - in silico prediction of the toxic (endocrine-disrupting) potential of drugs, chemicals and natural products. Two years and 2,000 compounds of experience: a progress report. Alternatives to Animal Experimentation 26(3): 167-76.

Voskoboynik A., Reznick A.Z., Rinkevich B. 2002. Rejuvenescence and extension of an urochordate life span following a single, acute administration of an anti-oxidant, butylated hydroxytoluene. Mechanisms of Ageing and Development 123(9): 1203-10. doi: 10.1016/s00476374(02)00002-7

Vrskova D., Modra H. 2012. Evaluation of the developmental toxicity of 2 phenoxyethanol and clove oil anaesthetics using the Frog Embryo Teratogenesis Assay: Xenopus (FETAX). Veterinarni Medicina 57(5): 245-50.

Watkins R.E., Wisely G.B., Moore L.B., Collins J.L., Lambert M.H., Williams S.P., Willson T.M., Kliewer S.A., Redinbo M.R. 2001. The human nuclear xenobiotic receptor PXR: structural determinants of directed promiscuity. Science 292(5525): 2329-33. doi: 10.1126/science.1060762

Watkins R.E., Maglich J.M., Moore L.B., Wisely G.B., Noble S.M., DavisSearles P.R., Lambert M.H., Kliewer S.A., Redinbo M.R. 2003. 2.1 angstrom crystal structure of human PXR in complex with the St. John's 
wort compound hyperforin. Biochemistry 42(6): 1430-8. doi: 10.1021/bi0268753

Weeks J.A., Guiney P.D., Nikiforov A.I. 2012. Assessment of the environmental fate and ecotoxicity of $n, n$-diethyl-m-toluamide (DEET). Integrated Environmental Assessment and Management 8(1): 120-34. doi: 10.1002/ieam. 1246

Wise J., Wise J.P., Sr. 2011. A review of the toxicity of chemical dispersants. Reviews on Environmental Health 26(4): 281-300. doi: 10.1515/reveh.2011.035

Zarn J.A., Bruschweiler B.J., Schlatter J.R. 2003. Azole fungicides affect mammalian steroidogenesis by inhibiting sterol 14 alpha-demethylase and aromatase. Environmental Health Perspectives 111(3): 255-61. doi: 10.1289/ehp.5785

Zhang Z.D., Burch P.E., Cooney A.J., Lanz R.B., Pereira F.A., Wu J.Q., Gibbs R.A., Weinstock G., Wheeler D.A. 2004. Genomic analysis of the nuclear receptor family: new insights into structure, regulation and evolution from the rat genome. Genome Research 14(4): 580-90. doi:

10.1101/gr.2160004 


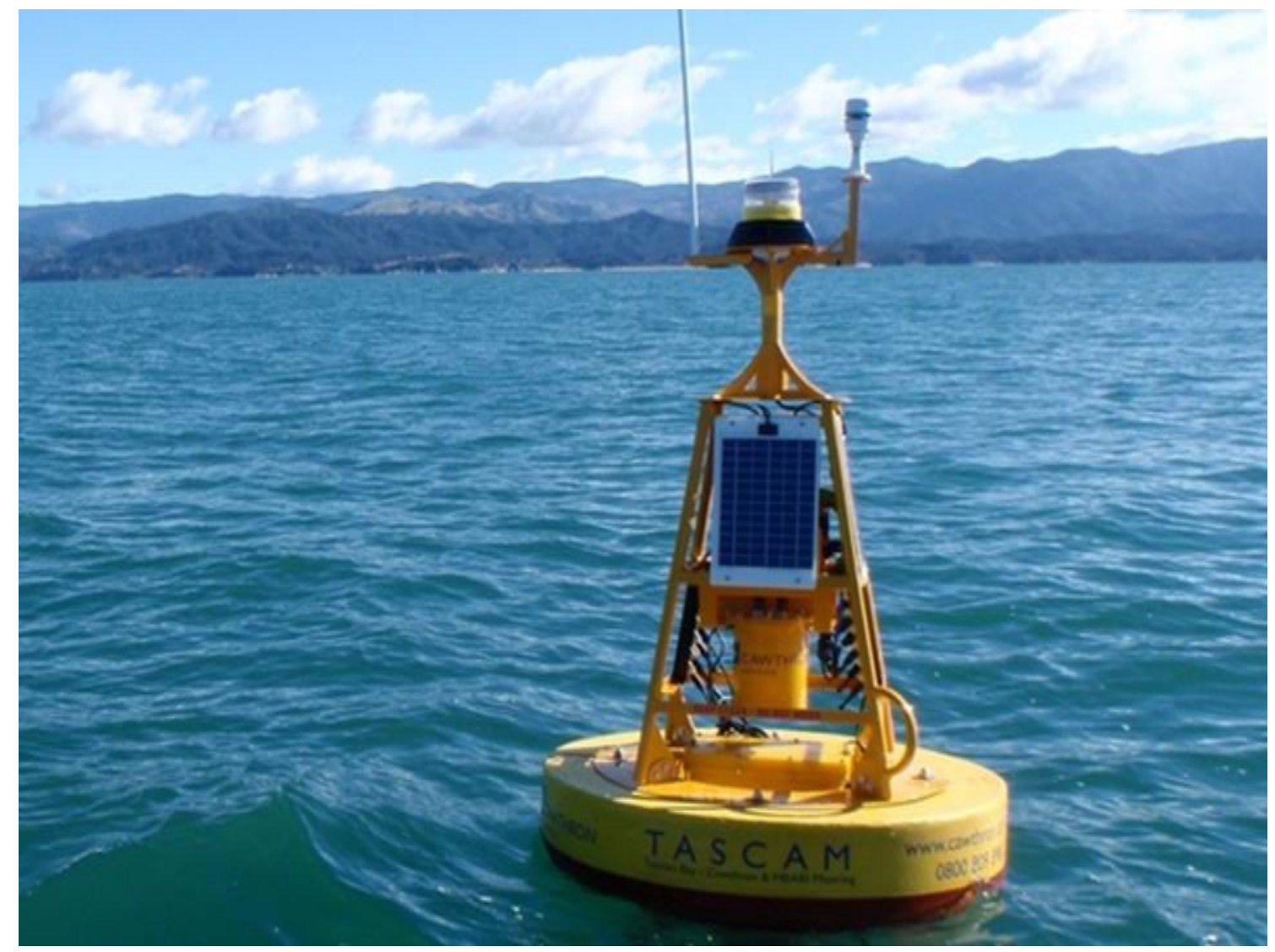

Coastal monitoring buoy used to remotely collect environmental data on the water quality of Tasman Bay, New Zealand.

Photo provided by Cawthron Institute. 



\section{CHAPTER SIX}

\section{Conclusions}

\subsection{Thesis synthesis}

Detailed discussions are provided at the end of each chapter of this thesis. The purpose of this section is to collate and summarise the major conclusions arising from the key findings and recommend areas for further research.

The research outcomes from this thesis demonstrate that a specific group of ligand-activated transcription factors, namely tunicate xenobiotic receptors, may provide a source of sensor elements that have been pre-moulded by natural selection for detecting bioactive chemicals. High intra-taxa genetic diversity within tunicate xenobiotic receptor genes, orthologues to vertebrate vitamin $D$ receptor (VDR) and pregnane $X$ receptor (PXR), was reported in Chapter Two. These data supported the notion that tunicate VDR/PXR receptors might enhance binding of dietary exogenous bioactives/toxins typically encountered by these organisms. This provided the foundation for the development of a generic bioassay for assessing the activity of these biological compounds (Chapter Three). Recombinant yeast strains were developed in which liganddependent activation of tunicate VDR/PXRs was transduced into easily quantifiable phenotypic changes in the yeast strains. In this thesis, I showed that tunicate yeast bioassays have the potential for detecting microalgal biotoxins (Chapter Three), bioactive compounds that may provide templates for drug development (Chapter Four) and environmental contaminants (Chapter Five).

Previously, sequence comparisons between vertebrate taxa have suggested positive selection is acting on ligand-binding domain (LBD) coding sequences of PXR and its orthologues (Zhang et al. 2004; Krasowski et al. 2005). In Chapter Two, high intra-species sequence variation in VDR/PXR LBD coding sequences of two tunicates, Ciona intestinalis and Botryllus schlosseri, was reported. To 
characterise the effect of natural selection at the molecular level, nonsynonymous variation (i.e. amino acid changing) was compared to synonymous changes (McDonald \& Kreitman 1991) which indicated a strong purifying selection process. While this result was unexpected, recent studies have suggested that multiple evolutionary forces can affect amino acid substitution rates (Bromham 2009; Lourenco et al. 2013), with one critical factor being mutation. An elevated per-year mutation rate, due to a large effective population size and a short generation time, may explain the extremely highly levels of genetic diversity within tunicates (Kimura 1983; Lynch 2008; Tsagkogeorga et al. 2012; Berná \& Alvarez-Valin 2014). In addition, the rate of environmental change and dimensionality of the phenotypic space (organism complexity) can affect adaptive rates in some species (Lourenco et al. 2013). While it is difficult to evaluate how these factors translate to changes in fitness, it is assumed that fast evolving tunicates are able to adapt rapidly to environmental challenges (Tsagkogeorga et al. 2012). Adaptive genetic signatures (e.g. mutation rates over time) of tunicates can be very informative of changes to our marine environment because the influence of environmental factors can lead to accumulation of genetic variation (Dalziel et al. 2009; Barrett \& Hoekstra 2011). One such factor is climate change which imposes novel selection pressures on organisms by altering the abiotic and biotic environmental conditions. Although studies demonstrating genetic adaptation to climate change-mediated selection are still scarce (Merila 2012; Merila \& Hendry 2014), the frequencies of some well-characterised genetic polymorphisms in Drosophila species have been shown to shift with climate change (Umina et al. 2005; Balanya et al. 2006).

Alternatively, a selective advantage in the marine environment may be facilitated through different processes. Ciona intestinalis VDR/PXR protein variants consisting of a DNA-binding domain (DBD) but lacking a LBD were identified in this study. The persistence of these variants may indicate constitutive expression of detoxification genes, thus providing a biochemical protection mechanism in a ligand-independent manner. In addition, tunicate genomes encode multiple VDR/PXR paralogues, with each subtype potentially binding a differing range of ligand structures (Dehal et al. 2002; Denoeud et al. 2010; Voskoboynik et al. 2013). 
In previous studies, Fidler et al. (2012) demonstrated that LBDs from a tunicate xenobiotic receptor can be activated by microalgal biotoxins when expressed in mammalian cell lines. In Chapter Three, C. intestinalis and B. schlosseri VDR/PXRa LBDs were functionally expressed in recombinant yeast (Saccharomyces cerevisiae) strains as chimeric proteins. Three biotoxins, produced by microalgae that are expected to be part of the tunicate diet, were identified as putative VDR/PXRa LBD ligands. This supported the idea that the natural tunicate $V D R / P X R \alpha$ receptor may be activated by exogenous compounds relevant to its marine environment. The sensitivity of the yeast bioassay towards both microalgal biotoxins and synthetic compounds appeared to depend on several factors, such as the organic solvent used, duration of exposure and type of recombinant protein expressed (e.g. C. intestinalis verses B. schlosseri VDR/PXRa). This implies that assay sensitivity can be improved by changing or varying these factors (Eichbaum et al. 2014). Assay sensitivity may also be improved by using a different type of reporter gene. For example, yeast-enhanced green fluorescence protein and luciferase reporter assays are more sensitive than lacZ (Fan \& Wood 2007) and these could be investigated in the future for this bioassay.

The development of robust high-throughput screening (HTS) assays for identifying natural marine bioactive compounds for pharmaceutical purposes is technically challenging (Martins et al. 2014). Chapter Four, showed that the yeast bioassays were highly sensitive towards a small number of marine and terrestrial bioactive compounds. These yeast bioassays have a number of characteristics that may be beneficial for pharmaceutical screening. The orthology of the tunicate VDR/PXRa with vertebrate PXR (Ekins et al. 2008) suggests that compounds active in the yeast bioassay may also affect vertebrate physiology. This is an important consideration when developing bioassays for drug discovery, because the natural biological activities of putative drug compounds influence their potential medical applications (Imhoff et al. 2011; Martins et al. 2014). The yeast bioassays are also well-suited for HTS, which is the most widely used screening approach for the identification of novel drug lead compounds (Hughes et al. 2011; Martins et al. 2014). Therefore, if sensitivity and reliability of yeast bioassays can be improved in future, these assays may provide a template for the development of cost- 
effective methods for the detection of bioactive compounds during the early phases of drug discovery.

Recently, a new class of environmental pollutants, termed emerging contaminants (ECs), has received scientific and public interest because they may pose a health risk to many organisms, including humans (Gavrilescu et al. 2015). In Chapter Five, a wide range of synthetic chemicals, such as pesticides, pharmaceuticals and surfactants, were shown to activate the tunicate yeast bioassays. Although the responses for some of the aforementioned compounds were highly variable, the in vitro bioassay has the potential to be a reliable and inexpensive tool for monitoring chemical mixtures such as surfactants (e.g. oil dispersants; Bandele et al. 2012). By evaluating data generated in the yeast in vitro bioassay, it may be possible to predict the effects of environmental surfactants on coastal invertebrates, especially when combined with in vivo toxicity studies. This is of particular importance since most in vitro bioassays focus on assessing the physiological effects that environmental contaminants can cause in humans, while overlooking the far-reaching impacts on aquatic organisms.

\subsection{Future research}

The data presented in this thesis provide a foundation for further investigations into the molecular evolution, population genetics and function of tunicate xenobiotic receptors thought to be involved in the detection of marine bioactive compounds. If dietary bioactive xenobiotics act as selective agents in shaping the structure of tunicate VDR/PXR LBDs, then it is reasonable to expect that exposure to different dietary toxins in species occupying different ecological niches will be reflected in intra-species sequence variation in VDR/PXR orthologues. This is supported by the fact that geographical location and exposure to toxic compounds can generate different mutational variants of a species (Whitehead et al. 2003). Genetic analysis of tunicate VDR/PXR orthologues from populations from different geographical locations and ecological niches may allow identification of targets of adaptive natural selection 
(Li et al. 2008). Another possible approach could involve measuring differences in gene expression between taxa, by considering the hypothesis that at least some differences in the pattern or level of transcript abundance represent phenotypic traits contributing to adaptation (Ranz \& Machado 2006).

The role of VDR/PXR orthologues in intact, living tunicates has not been determined and the toxicity of microalgal biotoxins towards tunicates is currently unknown. The development of a tunicate in vivo bioassay, using $C$. intestinalis adults, would assist in addressing these knowledge gaps. These assays would complement current in vivo bioassays used to investigate toxicological effects of chemicals on metamorphosis and larval development (Bellas et al. 2003; Cahill et al. 2012). Another interesting approach for studying the functional role of tunicate xenobiotic receptors could involve selective breeding of $C$. intestinalis adults that are homozygous for the VDR/PXR allelic variants that produce VDR/PXR proteins lacking a LBD. Exposure of these organisms to a range of known xenobiotics, including microalgal biotoxins, may reveal if VDR/PXR target genes are constitutively transcribed providing an advantage over ligandactivated transcription.

The tunicate yeast bioassay developed in this study represents a promising template for the development of a broad-spectrum screening tool which could be used for routine microalgal biotoxin detection and bioprospecting. To assess the bioassay's reliability, extracts of toxic shellfish should be tested for presence of microalgal biotoxins. It would be particularly useful to compare these in vitro data with detailed chemical liquid chromatography-mass spectrometry analyses to confirm the yeast bioassay's value in an applied context. If this can be achieved, then the yeast bioassay has the potential to significantly reduce the costs associated with current chemistry-based methods and may assist in the detection of unknown toxins (Humpage et al. 2010; Nicolas et al. 2014). This novel method may also be used by those with little expertise and who have only minimal capital to invest in equipment (i.e. developing countries).

To test the reliability of the yeast bioassay for bioprospecting, libraries of natural products and environmental extracts should be analysed for bioactive compounds and the resulting data compared with established ligand-binding assays used during drug discovery (Pinne \& Raucy 2014). Compound libraries 
are usually composed of crude extracts, simplified extract fractions and pure compounds for a well-balanced drug discovery programme (Kingston 2011). Crude extracts are complex mixtures of several compounds that may have synergistic interaction causing false negative read-outs (Parker et al. 2010). Due to the broad-spectrum activity of xenobiotic receptors, interference of other compounds that bind to the receptor is likely to cause false positives. Screening pre-fractionated libraries would be an effective strategy to avoid these problems (Carter 2011). To be applied in bioprospecting, the yeast bioassay needs to be amenable to HTS formats (Hughes et al. 2011). All yeast bioassay experiments conducted in this thesis were performed in 96-well plate formats which have the potential to be miniaturised to high-throughput 1536-well microplate formats (Rajasarkka \& Virta 2011). The yeast bioassay could easily be adapted to robotic liquid handling, one of the main requirements for HTS. Assay miniaturisation and robotic liquid handling would likely reduce inter-plate variability, thus increasing the over-all reliability of the yeast bioassay. In addition, the $S$. cerevisiae host strain allows for refinements in the detection specificities and sensitivities of the yeast bioassay using laboratory-based in vitro mutagenesis and directed evolution (Chen \& Zhao 2003). Using this approach VDR/PXR LBD variant sequences, generated by in vitro mutagenesis, can be selected for enhancement of growth rates in the presence of a cognate ligand. For example, Chen \& Zhao (2003) used random in vitro mutagenesis combined with directed evolution to generate novel variants of the human estrogen receptor $(E R \alpha)$ LBD that had significantly modified ligand-binding properties.

To be used in environmental monitoring, it is crucial that the yeast bioassays meet analytical goals and/or regulatory guidelines. Most ECs generally occur in surface and ground waters at very low concentrations (e.g. $\mathrm{ng} / \mathrm{L}$ to $\mu \mathrm{g} / \mathrm{L}$; Stewart et al. 2014). Thus, the relatively low sensitivity and reliability of the tunicate yeast bioassays towards a wide range of environmental contaminants is currently the major limitation. These limitations could be overcome by developing biosensors that are based on tunicate VDR/PXR receptors (Rodriguez-Mozaz et al. 2006; Holdgate et al. 2010; Senveli \& Tigli 2013). The main advantage of biosensors is their cell-free nature, thereby removing the limitations associated with cell-based bioassays, such as the need for test 
compounds to cross cell membranes (Norcliffe et al. 2013). Biosensors typically consist of a macromolecule immobilised on a surface via either covalent or strong non-covalent bonds (Fechner et al. 2010). An important consideration is that such attachments should not significantly influence the natural structure of the macromolecule or change its functionality in unpredictable ways (Fechner et al. 2010). A wide range of techniques exist to detect and quantify interactions between the immobilised macromolecules and potential ligands including; calorimetric, acoustic, electrical, magnetic and optical sensing techniques (Senveli \& Tigli 2013). Development of such biosensor techniques utilising tunicate VDR/PXR $\alpha$ receptors as the sensor element may result in a more universal and generic detection system compared to yeast-based bioassays. Numerous nuclear receptor (NR) LBD-based biosensors have been developed for use in environmental monitoring (Dutta et al. 2007) and drug development (Fechner et al. 2010; Raucy \& Lasker 2013). Among the established xenobiotic receptors, the human PXR LBD has been used successfully as the sensor element in a number of differing biosensor formats (Moore et al. 2000; Hill et al. 2011; Lin et al. 2014). These biosensors have confirmed a number of human PXR ligands such as; hyperforin, clotrimazole, ginkgolide A, SR12813 and 5bpregnane-3,20-dione (Jones et al. 2000; Moore et al. 2000; Lin et al. 2014). The successful development of human PXR LBD-based biosensors supports the theoretical feasibility of using tunicate VDR/PXR LBDs in biosensor formats to screen for bioactive compounds. If routine production of correctly folded and soluble tunicate VDR/PXR LBDs can be achieved, they could be used in affinity chromatography to identify and isolate novel VDR/PXR ligands, as has recently been reported for the human PXR LBD (Dagnino et al. 2014).

Determining the three dimensional structure of the $C$. intestinalis and $B$. schlosseri VDR/PXRa receptor represents another important area of future research. Crystallisation of the VDR/PXR LBD in complex with a putative ligand may provide important insights into the permissiveness of these receptors as has been reported for vertebrate PXR (Wallace \& Redinbo 2013; Wu et al. 2013). Previous studies suggested that the $C$. intestinalis VDR/PXR LBD may have narrower ligand selectivity when compared to vertebrate, particularly human, PXRs (Ekins et al. 2008; Fidler et al. 2012). While only structural data can address these questions, it would be interesting to study the function of the 
second $C$. intestinalis VDR/PXR orthologue (VDR/PXR $\beta$ ) using the recombinant yeast strains developed in this study. This gene had a higher genetic diversity than VDR/PXR $\alpha$, and the predicted protein sequence suggested a larger LBD than its VDR/PXRa paralogue. Therefore, the VDR/PXR $\beta$ LBD may be able to bind larger compounds or a complete different set of ligands.

Due to the highly modular structure of the tunicate yeast bioassay, the VDR/PXR LBDs could be exchanged with virtually any suitable PXR orthologues from species other than $C$. intestinalis and B. schlosseri. By selecting, on the basis of taxonomy and ecology, the organism to source PXR LBDs from, it may be possible to tailor bioassays to search for bioactive compounds from differing sources. Ligand binding-domains of potential PXR orthologues have been identified in the genomes of other tunicates (e.g. six VDR/PXR-like genes were identified in Oikopleura diocia; Denoeud et al. 2010). There is no a priori reason why taxon selection should be restricted to the tunicates. For example, filter-feeding bivalves use a somewhat different mechanism for filtering seawater than tunicates. Therefore, these two groups ingest different profiles/size-ranges of marine microorganisms, thus being exposed to different toxins (Roje-Busatto \& Ujević 2014). Some bioactive chemicals may be produced by marine organisms that adhere to hard surfaces. Benthic microalgae, such as the dinoflagellate Gambierdiscus toxicus can produce highly toxic compounds (e.g. ciguatera-associated toxins; Parsons et al. 2011). To detect such toxins, PXR LBDs from surface-grazing animals are likely to be more suitable for the yeast bioassay (Richter \& Fidler 2014). 


\subsection{References}

Balanya J., Oller J.M., Huey R.B., Gilchrist G.W., Serra L. 2006. Global genetic change tracks global climate warming in Drosophila subobscura. Science 313(5794): 1773-5. doi: 10.1126/science.1131002

Bandele O.J., Santillo M.F., Ferguson M., Wiesenfeld P.L. 2012. In vitro toxicity screening of chemical mixtures using HepG2/C3A cells. Food and Chemical Toxicology 50(5): 1653-9. doi: 10.1016/j.fct.2012.02.016

Barrett R.D.H., Hoekstra H.E. 2011. Molecular spandrels: tests of adaptation at the genetic level. Nature Reviews Genetics 12(11): 767-80. doi: $10.1038 / \mathrm{nrg} 3015$

Bellas J., Beiras R., Vazquez E. 2003. A standardisation of Ciona intestinalis (Chordata, Ascidiacea) embryo-larval bioassay for ecotoxicological studies. Water Research 37(19): 4613-22. doi: 10.1016/s00431354(03)00396-8

Berná L., Alvarez-Valin F. 2014. Evolutionary genomics of fast evolving tunicates. Genome Biology and Evolution. doi: 10.1093/gbe/evu122

Bromham L. 2009. Why do species vary in their rate of molecular evolution? Biological Letters 5(3): 401-4. doi: 10.1098/rsbl.2009.0136

Cahill P., Heasman K., Jeffs A., Kuhajek J., Mountfort D. 2012. Preventing ascidian fouling in aquaculture: screening selected allelochemicals for anti-metamorphic properties in ascidian larvae. Biofouling 28(1): 39-49. doi: 10.1080/08927014.2011.648624

Carter G.T. 2011. Natural products and Pharma 2011: strategic changes spur new opportunities. Nature Product Reports 28(11): 1783-9. doi: 10.1039/c1np00033k

Chen Z.L., Zhao H.M. 2003. A highly efficient and sensitive screening method for trans-activation activity of estrogen receptors. Gene 306: 127-34. doi: 10.1016/s0378-1119(03)00431-1

Dagnino S., Bellet V., Grimaldi M., Riu A., Ait-Aissa S., Cavailles V., Fenet H., Balaguer P. 2014. Affinity purification using recombinant PXR as a tool to characterize environmental ligands. Environmental Toxicology 29(2): 207-15. doi: 10.1002/tox.20787

Dalziel A.C., Rogers S.M., Schulte P.M. 2009. Linking genotypes to phenotypes and fitness: how mechanistic biology can inform molecular ecology. Molecular Ecology 18(24): 4997-5017. doi: 10.1111/j.1365294X.2009.04427.x

Dehal P., Satou Y., Campbell R.K., Chapman J., Degnan B., De Tomaso A., Davidson B., Di Gregorio A., Gelpke M., Goodstein D.M.et al. 2002. The draft genome of Ciona intestinalis: insights into chordate and vertebrate origins. Science 298(5601): 2157-67. doi: 10.1126/science.1080049

Denoeud F., Henriet S., Mungpakdee S., Aury J.-M., Da Silva C., Brinkmann H., Mikhaleva J., Olsen L.C., Jubin C., Canestro C.et al. 2010. Plasticity of animal genome architecture unmasked by rapid evolution of a pelagic tunicate. Science 330(6009): 1381-5. doi: 10.1126/science.1194167

Dutta P., Hill K., Datskos P.G., Sepaniak M.J. 2007. Development of a nanomechanical biosensor for analysis of endocrine disrupting chemicals. Lab on a Chip 7(9): 1184-91. doi: 10.1039/B704723A 
Eichbaum K., Brinkmann M., Buchinger S., Reifferscheid G., Hecker M., Giesy J.P., Engwall M., van Bavel B., Hollert H. 2014. In vitro bioassays for detecting dioxin-like activity - application potentials and limits of detection, a review. Science of the Total Environment 487(0): 37-48. doi: 10.1016/j.scitotenv.2014.03.057

Ekins S., Reschly E.J., Hagey L.R., Krasowski M.D. 2008. Evolution of pharmacologic specificity in the pregnane $\mathrm{X}$ receptor. BMC Evolutionary Biology 8. doi: 10.1186/1471-2148-8-103

Fan F., Wood K.V. 2007. Bioluminescent assays for high-throughput screening. Assay and Drug Development Technologies 5(1): 127-36. doi: 10.1089/adt.2006.053

Fechner P., Gauglitz G., Gustafsson J.-A. 2010. Nuclear receptors in analytics - a fruitful joint venture or a wasteful futility? Trends in Analytical Chemistry 29(4): 297-305. doi: 10.1016/j.trac.2009.12.009

Fidler A.E., Holland P.T., Reschly E.J., Ekins S., Krasowski M.D. 2012. Activation of a tunicate (Ciona intestinalis) xenobiotic receptor orthologue by both natural toxins and synthetic toxicants. Toxicon 59: 365-72. doi: 10.1016/j.toxicon.2011.12.008

Gavrilescu M., Demnerova K., Aamand J., Agathos S., Fava F. 2015. Emerging pollutants in the environment: present and future challenges in biomonitoring, ecological risks and bioremediation. New Biotechnology 32(1): 147-56. doi: 10.1016/j.nbt.2014.01.001

Hill K.L., Dutta P., Long Z., Sepaniak M.J. 2011. Microcantilever-based nanomechanical studies of the orphan nuclear receptor pregnane $X$ receptor-ligand interactions. Journal of Biomaterials and Nanobiotechnology: 133-42. doi: 10.4236/jbnb.2011.22017

Holdgate G.A., Anderson M., Edfeldt F., Geschwindner S. 2010. Affinity-based, biophysical methods to detect and analyze ligand binding to recombinant proteins: matching high information content with high throughput. Journal of Structural Biology 172(1): 142-57. doi: 10.1016/j.jsb.2010.06.024

Hughes J.P., Rees S., Kalindjian S.B., Philpott K.L. 2011. Principles of early drug discovery. British Journal of Pharmacology 162(6): 1239-49. doi: 10.1111/j.1476-5381.2010.01127.x

Humpage A.R., Magalhaes V.F., Froscio S.M. 2010. Comparison of analytical tools and biological assays for detection of paralytic shellfish poisoning toxins. Analytical and Bioanalytical Chemistry 397(5): 1655-71. doi: 10.1007/s00216-010-3459-4

Imhoff J.F., Labes A., Wiese J. 2011. Bio-mining the microbial treasures of the ocean: new natural products. Biotechnology Advances 29(5): 468-82. doi: 10.1016/j.biotechadv.2011.03.001

Jones S.A., Moore L.B., Shenk J.L., Wisely G.B., Hamilton G.A., McKee D.D., Tomkinson N.C.O., LeCluyse E.L., Lambert M.H., Willson T.M.et al. 2000. The pregnane $X$ receptor: a promiscuous xenobiotic receptor that has diverged during evolution. Molecular Endocrinology 14(1): 27-39. doi: $10.1210 /$ me.14.1.27

Kimura M. 1983. The neutral theory of molecular evolution. first ed. Cambridge, Cambridge University Press. 208-33 p.

Kingston D.G. 2011. Modern natural products drug discovery and its relevance to biodiversity conservation. Journal of Natural Products 74(3): 496-511. doi: $10.1021 / \mathrm{np} 100550 \mathrm{t}$ 
Krasowski M.D., Yasuda K., Hagey L.R., Schuetz E.G. 2005. Evolution of the pregnane $\mathrm{X}$ receptor: adaptation to cross-species differences in biliary bile salts. Molecular Endocrinology 19(7): 1720-39. doi: 10.1210/me.2004-0427

Li Y.F., Costello J.C., Holloway A.K. 2008. "Reverse ecology" and the power of population genomics. Evolution 62: 2984-94. doi: 10.1111/j.15585646.2008 .00486

Lin W., Liu J., Jeffries C., Yang L., Lu Y., Lee R.E., Chen T. 2014. Development of BODIPY FL Vindoline as a novel and high-affinity pregnane $X$ receptor fluorescent probe. Bioconjugate Chemicals 18: 18. doi: $10.1021 / \mathrm{bc5002856}$

Lourenco J.M., Glemin S., Galtier N. 2013. The rate of molecular adaptation in a changing environment. Molecular Biology and Evolution 30(6): 1292-301. doi: $10.1093 / \mathrm{molbev} / \mathrm{mst026}$

Lynch M. 2008. The cellular, developmental and population-genetic determinants of mutation-rate evolution. Genetics 180(2): 933-43. doi: 10.1534/genetics.108.090456

Martins A., Vieira H., Gaspar H., Santos S. 2014. Marketed marine natural products in the pharmaceutical and cosmeceutical industries: tips for success. Marine Drugs 12(2): 1066-101. doi: 10.3390/md12021066

McDonald J.H., Kreitman M. 1991. Adaptive protein evolution at the Adh locus in Drosophila. Nature 351(6328): 652-4. doi:10.1038/351652a0

Merila J. 2012. Evolution in response to climate change: in pursuit of the missing evidence. Bioessays 34(9): 811-8. doi: 10.1002/bies.201200054

Merila J., Hendry A.P. 2014. Climate change, adaptation and phenotypic plasticity: the problem and the evidence. Evolutionary Applications 7(1): 1-14. doi: 10.1111/eva.12137

Moore L.B., Parks D.J., Jones S.A., Bledsoe R.K., Consler T.G., Stimmel J.B., Goodwin B., Liddle C., Blanchard S.G., Willson T.M.et al. 2000. Orphan nuclear receptors constitutive androstane receptor and pregnane $\mathrm{X}$ receptor share xenobiotic and steroid ligands. Journal of Biological Chemistry 275(20): 15122-7. doi: 10.1074/jbc.M001215200

Nicolas J., Hendriksen P.J.M., Gerssen A., Bovee T.F.H., Rietjens I. 2014. Marine neurotoxins: state of the art, bottlenecks and perspectives for mode of action based methods of detection in seafood. Molecular Nutrition and Food Research 58(1): 87-100. doi: 10.1002/mnfr.201300520

Norcliffe J.L., Alvarez-Ruiz E., Martin-Plaza J.J., Steel P.G., Denny P.W. 2013. The utility of yeast as a tool for cell-based, target-directed highthroughput screening. Parasitology 141(1): 8-16. doi: 10.1017/s0031182013000425

Parker C.N., Ottl J., Gabriel D., Zhang J.H. 2010. Advances in biological screening for lead discovery. Natural Product Chemistry for Drug Discovery. Cambridge, U.K., Royal Society of Chemistry.

Parsons M.L., Settlemier C.J., Ballauer J.M. 2011. An examination of the epiphytic nature of Gambierdiscus toxicus, a dinoflagellate involved in ciguatera fish poisoning. Harmful Algae 10(6): 598-605. doi: 10.1016/j.hal.2011.04.011

Pinne M., Raucy J.L. 2014. Advantages of cell-based high-volume screening assays to assess nuclear receptor activation during drug discovery. 
Expert Opinion on Drug Discovery 9(6): 669-86. doi: 10.1517/17460441.2014.913019

Rajasarkka J., Virta M. 2011. Miniaturization of a panel of high throughput yeast-cell-based nuclear receptor assays in 384-and 1536-well microplates. Combinatorial Chemistry and High Throughput Screening 14(1): 47-54. doi: 10.2174/1386207311107010047

Ranz J.M., Machado C.A. 2006. Uncovering evolutionary patterns of gene expression using microarrays. Trends in Ecology and Evolution 21(1): 29-37. doi: 10.1016/j.tree.2005.09.002

Raucy J.L., Lasker J.M. 2013. Cell-based systems to assess nuclear receptor activation and their use in drug development. Drug Metabolism Reviews 45(1): 101-9. doi: 10.3109/03602532.2012.737333

Richter I., Fidler A. 2014. Marine invertebrate xenobiotic-activated nuclear receptors: their application as sensor elements in high-throughput bioassays for marine bioactive compounds. Marine Drugs 12(11): 5590618. doi:10.3390/md12115590

Rodriguez-Mozaz S., Lopez de Alda M.J., Barcelo D. 2006. Biosensors as useful tools for environmental analysis and monitoring. Analytical and Bioanalytical Chemistry 386(4): 1025-41. doi: 10.1007/s00216-006-05743

Roje-Busatto R., Ujević I. 2014. PSP toxins profile in ascidian Microcosmus vulgaris (Heller, 1877) after human poisoning in Croatia (Adriatic Sea). Toxicon 79(0): 28-36. doi: 10.1016/j.toxicon.2013.12.014

Senveli S.U., Tigli O. 2013. Biosensors in the small scale: methods and technology trends. Nanobiotechnology 7(1): 7-21. doi: 10.1049/ietnbt.2012.0005

Stewart M., Olsen G., Hickey C.W., Ferreira B., Jelic A., Petrovic M., Barcelo D. 2014. A survey of emerging contaminants in the estuarine receiving environment around Auckland, New Zealand. Science of the Total Environment 468: 202-10. doi: 10.1016/j.scitotenv.2013.08.039

Tsagkogeorga G., Cahais V., Galtier N. 2012. The population genomics of a fast evolver: high levels of diversity, functional constraint and molecular adaptation in the tunicate Ciona intestinalis. Genome Biology and Evolution 4(8): 740-9. doi: 10.1093/gbe/evs054

Umina P.A., Weeks A.R., Kearney M.R., McKechnie S.W., Hoffmann A.A. 2005. A rapid shift in a classic clinal pattern in Drosophila reflecting climate change. Science 308(5722): 691-3. doi: 10.1126/science.1109523

Voskoboynik A., Neff N.F., Sahoo D., Newman A.M., Pushkarev D., Koh W., Passarelli B., Fan H.C., Mantalas G.L., Palmeri K.J.et al. 2013. The genome sequence of the colonial chordate, Botryllus schlosseri. eLife 2. doi: 10.7554/eLife.00569

Wallace B.D., Redinbo M.R. 2013. Xenobiotic-sensing nuclear receptors involved in drug metabolism: a structural perspective. Drug Metabolism Reviews 45(1): 79-100. doi: 10.3109/03602532.2012.740049

Whitehead A., Anderson S.L., Kuivila K.M., Roach J.L., May B. 2003. Genetic variation among interconnected populations of Catostomus occidentalis: implications for distinguishing impacts of contaminants from biogeographical structuring. Molecular Ecology 12(10): 2817-33. doi: 10.1046/j.1365-294X.2003.01933.x 
Wu B., Li S., Dong D. 2013. 3D structures and ligand specificities of nuclear xenobiotic receptors CAR, PXR and VDR. Drug Discovery Today 18(1112): 574-81. doi: 10.1016/j.drudis.2013.01.001

Zhang Z.D., Burch P.E., Cooney A.J., Lanz R.B., Pereira F.A., Wu J.Q., Gibbs R.A., Weinstock G., Wheeler D.A. 2004. Genomic analysis of the nuclear receptor family: new insights into structure, regulation and evolution from the rat genome. Genome Research 14(4): 580-90. doi: 10.1101/gr.2160004 


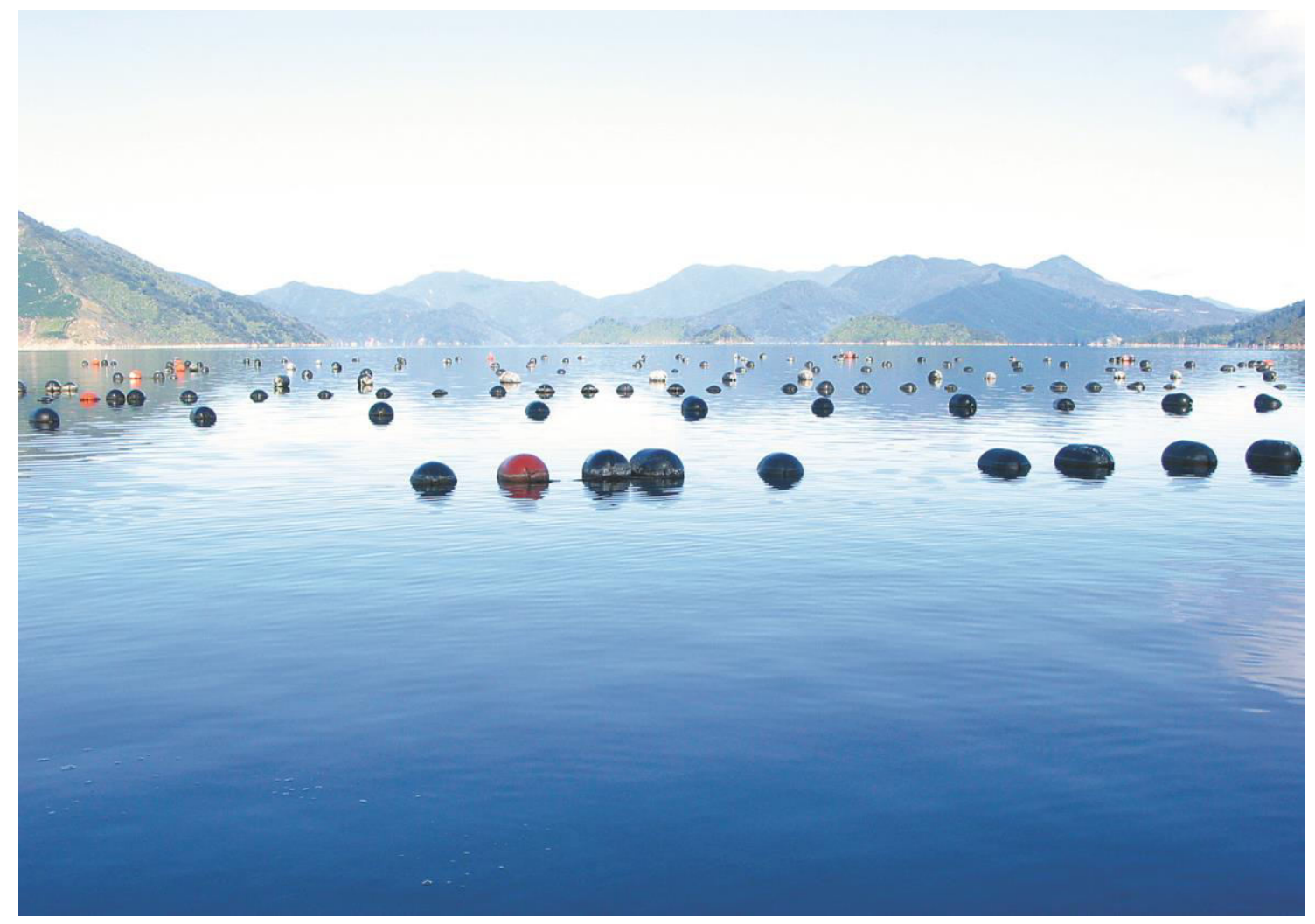

Aquaculture buoys in Brightlands Bay, Tawhitinui Reach, Marlborough Sounds, New Zealand. Photograph provided by Cawthron Institute. 


\section{APPENDIX ONE}

Appendix 1 Primers used for amplification of tunicate xenobiotic receptor orthologues.

(A) Primers used for the amplification of Ciona intestinalis vitamin $\mathrm{D}$ receptor/pregnane $\mathrm{X}$ receptor $\alpha(C N D R / P X R \alpha), C$. intestinalis VDR/PXR $\beta$ (CNDR/PXR $\beta$ ), C. intestinalis actin (Ciactin) and Botryllus schlosseri VDR/PXR $(B s V D R / P X R \alpha)$ partial coding sequences in polymerase chain reactions (PCRs). (B) Primers used for quantitative polymerase chain reactions (qPCR).

A

\begin{tabular}{|c|c|c|c|c|}
\hline Gene & GenBank acc. no. & & Primer sequence (5'-3') & Amplicon (bp) ${ }^{\mathrm{a}}$ \\
\hline \multirow[t]{2}{*}{ CIVDR/PXRa } & NM 001078379 & Forward & AAGTATTTCTGATCCATCGCTGG & 1326 \\
\hline & & Reverse & GTGGGTTGAACTTCTTTAACAAGAGG & \\
\hline \multirow[t]{2}{*}{$C N D R / P X R \beta$} & NM 001044366 & Forward & ATGTCAAATCCTCAAGGCCCATC & $1569^{b}$ \\
\hline & & Reverse & CATGATGTTTTGAGCGAATAGC & \\
\hline \multirow[t]{2}{*}{ BsVDR/PXRa } & Not available & Forward & TGCGARGGCTGYAAAGGITTYTTCAG & 1006 \\
\hline & & Reverse & CACGTTGGGCATACATTCAAATAC & \\
\hline \multirow[t]{2}{*}{ Ciactin } & $\underline{\text { AJ297725 }}$ & Forward & CTTAGGCAGTTTTAATGCAAGCG & 1454 \\
\hline & & Reverse & TAGCAGCTGAAGCCGGTTTAGGAA & \\
\hline
\end{tabular}


Appendix 1 (cont.) Primers used for amplification of tunicate xenobiotic receptor orthologues.

B

\begin{tabular}{|c|c|c|c|c|c|}
\hline Transcript & GenBank acc. no. & & Primer sequence $\left(5^{\prime}-3^{\prime}\right)$ & Amplicon (bp) ${ }^{\mathrm{a}}$ & Reference \\
\hline \multirow[t]{2}{*}{ CIVDR/PXRa } & NM 001078379 & Forward & GTTCACTTCACCGACATTATGGAG & 103 & This study \\
\hline & & Reverse & CGCGATTTGATCTTTTAGGTCC & & \\
\hline \multirow[t]{2}{*}{ CINDR/PXR $\beta$} & $\underline{\text { NM } 001044366}$ & Forward & GCTAAACAACTTTTCCAACATTTC & 100 & This study \\
\hline & & Reverse & GGATAGTTGGACAAACTGTGGTA & & \\
\hline \multirow[t]{2}{*}{ Ci actin } & $\underline{\text { AJ297725 }}$ & Forward & СTTCCTGACGGACAGGTTATCACC & 213 & Kulman, et al. 2006 \\
\hline & & Reverse & TAGCAGCTGAAGCCGGTTTAGGAA & & Kulman, et al. 2006 \\
\hline
\end{tabular}

Abbreviations: Ci, Ciona intestinalis; Bs, Botryllus schlosseri.

${ }^{\text {a }}$ Predicted amplicon size in base pair (bp).

${ }^{\mathrm{b}} \mathrm{PCR}$ generated a longer CNDR/PXR $\beta$ amplicon than predicted by the primer positions in the reference sequence ( $\underline{\mathrm{NM} 001044366)}$. 


\section{APPENDIX TWO}

Appendix 2 Conserved domain predictions in three tunicate xenobiotic receptor protein sequences generated in this study. Predicted locations of DNA-binding domain (DBD) and ligand-binding domain (LBD) within the Ciona intestinalis vitamin D receptor/pregnane $X$ receptor (CNDR/PXRa, KC561370), $C$. intestinalis VDR/PXR $(C N D R / P X R \beta, \underline{K C 561371)}$ ) and Botryllus schlosseri VDR/PXRa (BsVDR/PXRa, KC561372) conceptual proteins using the Simple Modular Architecture Research Tool (SMART; Letunic et al. 2012), the National Center for Biotechnology Information (NCBI) Conserved Domain (CD) database (NCBI CD; Marchler-Bauer et al. 2011) and the Protein Families database (Pfam; Punta et al. 2012).

\begin{tabular}{lllllll}
\hline & SMART & \multicolumn{3}{c}{ NCBI CD } & Pfam & \\
& DBD & LBD & DBD & LBD & DBD & LBD \\
\hline CNDR/PXRa & $56-127$ & $244-402$ & $59-130$ & $238-409$ & $57-126$ & $223-414$ \\
CiVDR/PXR $\beta$ & $1-59$ & $320-480$ & $2-88$ & $312-480$ & $1-58$ & $305-480$ \\
BsVDR/PXRa & $2-42$ & $153-311$ & $1-44$ & $145-311$ & $1-40$ & $135-325$ \\
\hline
\end{tabular}




\section{APPENDIX THREE}

VDR_human

VDR_mouse

VDR_rhesus

VDR_cow

VDR rat

VDR_xenopus_laevis

VDR_stickleback

VDR_medaka

VDR_zebrafis

VDR_lamprey

VDR_quail

VDR_halibut

VDR_salmon

PXR human

PXR mouse

PXR rat

PXR_rabbit

PXR chicken

PXR rhesus

PXR medaka

PXR xenopus tropicalis

BXRā xenopus

BXRb xenopus

PXR zebrafish

PXR_opossum

PXR_cow

PXR_chimpanzee

VDR/PXRa_ciona_(NP_001071847)

VDR/PXRb_ciona_(NP_001037831)

VDR/PXRa_ciona_(KC561370)

VDR/PXRb ciona (KC561371)

VDR/PXRa_botrulilus_(KC561372)

CAR_human

CAR mouse

CAR rat

CAR rhesus

CAR chimpanzee

CAR_COW
30

40

50

70

100

50 RICGVCGDRATGFHFNAMTCEGCKGFFRR--SMKRKALFTCPFNG-DCRITKDNRRHCOACRLKRCVDIGMMKDOIVLIKSSAIEVIMLRSNOS ETIDDM RICGVCGDRATEHF

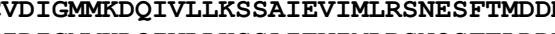
RICGVCGDRATGH RICGVGDAT

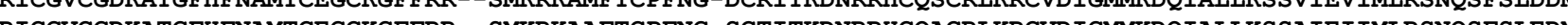
RICGVGDAI FFFAM CEGCKGFRR--SMKRKAAFICPFNG-SCIIIKDNRRHCQACRLKRCVDIGMMDQIALLKSSAIEIIMLRSNQSFSLEDM RICGVCGDKAIGFHFAMICEGCKGFFRRRRSMKRKASFTCPFNG-SCNITKDNRRHCQACRLKRCIDIGMMRDQIALLKSSAIEIIMLRSNQSFSLEDM PICGVCGDKATGFHFNAMTCEGCKGFFRR--SMKRKASFTCPFNG-NCTITKDNRRHCQACRLKRCIDIGMMKDQIALLKSSAIEIIMLRSNQSFSLEDM KVCGVCGDKATGYHFNAMTCEGCKGFFRR--SMKRSASFTCPFEG-KCNITKDNRRHCQACRLKRCRDIGMMKDQISLLKASAIEIIILRSNESFTMEDN RICGVCGDRATGFHFNAMTCEGCKGFFRR--SMKRKAMFTCPFSG-DCKITKDNRRHCQACRLKRCVDIGMMKDQIALLKSSAIEVIMLRSNQSFTMEDM RICGVCGDKATGFHFNAMTCEGCKGFFRR--SMKRKASFTCPFNG-SCTITKDNRRHCQACRLKRCIDIGMMKDQIALLKSSAIEIIMLRSNQSFSLEDM RICGVCGDKATGFHFNAMTCEGCKGFFRR--SMKRKASFTCP FNG-SCTITKDNRRHCQACRLKRCVDIGMMKDQTALLKSSAIEIIMLRSNQSFNPEDM QICRVCGDKATGYHFNVMTCEGCKGFFRR--AMKRNARLRCPFRKGACEITRKTRRQCQACRLRKCLESGMKKDQISLLKGAAFELCQLRFNTVFNAETG QICRVCGDKANGYHFNVMTCEGCKGFFRR--AMKRNVRLRCPFRKGTCEITRKTRRQCQACRLRKCLESGMKKDQISLLKGATFEMCILRFNTMFDTETG QICRVCGDKANGYHFNVMTCEGCKGFFRR--AMKRNVRLRCPFRKGTCEITRKTRRQCQACRLRKCLESGM--DQISLLKGATFEMCILRFNTMFDTETG OTCRVCGDKANGYHFNVLTCEGCKGFFRR--TVKRNARLRCPFRKGACEITRKTRROCOACRLRKCLESGM--DOISLLKGATLELCLLRFNTVFNAETG KVCAVCGDRATGYHFHVMSCEGCKGFFRR--SILKGVHFTCPFTR-SCPITKAKRROCOACRLOKCLDVGM--DOISLLKGATLGICOIOFNTVFNEETM OICRVCGDKATGYHFNVMTCEGCKGFFRR--AMKRNARLRCPFRKGACEITRKTRROCOACRLRKCLESGMKKDOISLIKGATFELCOLRFNTVFNAETG KACGVCGDIAKGYHFNAIC KICRACGDRATGYHF KICRAC KICRACGDRAT KICRACGDAIGYH KICQVCGDKSIGIFNATCEGCKGECRR O QICRVCGDKATGYHFNVMTCEGCKGFFRR--AMKRNAQPRCPFRKGTCEITQKTRRQCQACRLRKCLESGMRKDQISLLKGAAFELCQLRFNTVFNAETR

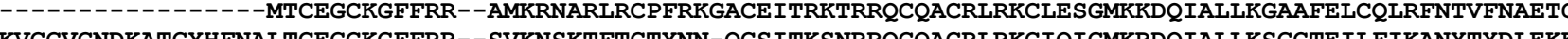
KVCGVCNDKATGYHFNALTCEGCKGFFRR--SVKNSKTFTCTYNN-QCSITKSNRRQCQACRLRKCIQIGMKRDQIALLKSGCTEILFIKANYTYDLEKK ----------MHFGAITCEGCKGFFRR--SVKKNASF SCAFEK-KCE INKNNRKHCQACRFNACLAAGMNSDQIVLLRGGCLEMLVLRSYFAFSCNEI KVCGVCNDKATGYHFNALTCEGCKGFFRR--SVKNSKTFTCTYNN-QCSITKSNRRQCQACRLRKCIQIGMKRDQIALLKSGCTE ILFIKANYTYDLEKK KICVVCNDKATGMHFGAITCEGCKGFFRR--SVKKNASFSCAFEK-KCEINKNNRKHCQACGFNACLAAGMNSDQIVLLRGGCLEMLVLRSYFAFSCNEN

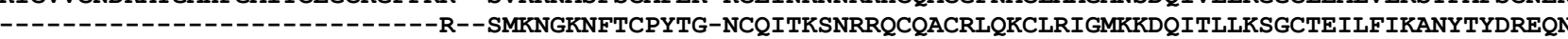
RNCVVCGDQATGYHFNALTCEGCKGFFRR--TVSKSIGPTCPFAG-SCEVSKTQRRHCPACRLOKCLDAGMRKDQISLLKGAAVEICHIVLNTTFCLQTQ RNCVVCGDRATGYHFHALTCEGCKGFFRR--TVSKTIGPICPFAG-RCEVSKAQRRHCPACRLQKCLNVGMRKDQISLLKGAAVEILHISLNTTFCLQTE RNCVVCGDRATGYHFHALTCEGCKGFFRR--TVSKTIGPICPFAG-RCEVSKAQRRHCPACRLQKCLNVGMRKDQISLLKGAAVEILHISLNTTFCLQTQ RNCVVCGDOATGYHFNALTCEGCKGFFRR--TVSKSIGPTCPFAG-SCEVSKIQRRHCPACRLQKCLDAGMRKDQISLLKGAAVE ICHIVLNTTFCLOTQ RNCVVCGDOATGYHFNALTCEGCKGFFRR--TVSKS IGPTCPFAG-SCEVSKTORRHCPACRLOKCLDAGMRKDOISLIKGAAVEICHIVINTTFCLOTQ RNCAVCGDRATGYHFHALTCEGCKGFFRR--TVNKSTSLTCPFAG-SCEVNKAQRRHCPACRLQKCLDAGMKKDQISLLKGAAIEICHIALNTTFCLQTQ 
CAR_opossum

seal

CAR_baikal_seal

CAR_dog

CAR_pig

ECR ${ }^{-}$silkworm

ECR/FXR_sea_urchin
RSCVVCGDRATGYHFHALTCEGCKGFFRR--TINKGMGLTCPFDQ-CCEVSKNQRRHCPACRLQKCLDVGMKKDQISLLKGAALEICHIELNTIFCPQSO RSCMVCGDRATGYHFHALTCEGCKGFFRR--TVSKNTGLTCPFAG-NCKVNKAQRRHCPACRLQKCLDAGMKKDQISLLKGAAVEICHIALNTTFCLQTR RSCMVCGDRATGYHFHALTCEGCKGFFRR--TVSKNTGLTCPFAG-SCKVNKAORRHCPACRLOKCLDAGMKKDQISLLKGAAVE ICHIALNTTFCLQTR RNCMVCGDRATGYHFHALTCEGCKGFFRR--TVSKSTGLTCPFAG-SCKVNKAQRRHCPACRLQKCLDAGMRKDQISLLKGAAVEICHIALITFCLQIR

RNCAVCGDRATGYHFHALTCEGCKGFFRR--IVNKTSLICPFAG-SCKVNKAQRRHCPACRLQKCLDAGMKKDQISLLKGAAVEICQIVLNTTFCLQTQ

ELCLVCGDRASGYHYNALTCEGCKGFFRR--SITKNAVYQCKYGN-NCDIDMYMRRKCQECRLKKCLSVGMRPDQITLLKACSSEVMLRGARKYDVKTD

ELCLVCGDRASGYHYNALTCEGCKGFFRR--SVTKNAVY ICKFGH-ACEMDMYMRRKCQECRLKKCLAVGMRPDQITLLKASSSEVMMLRVARRYDAASD ELCLVCGDRASGFHYNALSCEGCKGFFRR--SITKNAKYNCTRGG-NCEMDMYMRRKCQECRLRKCREVGMLADQILLLKGSAIEVIMLRVALRYDRELD 
VDR_human

VDR_mouse

VDR_rhesus

VDR_chimpanzee

VDR_COW

VDR_rat

VDR_xenopus_laevis

VDR_stickleb̄ack

VDR medaka

VDR_zebrafish

VDR lamprey

VDR_quail

VDR_halibut

VDR_salmon

PXR_human

PXR_mouse

PXR_rat

PXR_rabbit

PXR_chicken

PXR_rhesus

PXR_medaka

PXR_xenopus_tropicalis

BXRa_xenopus

$\mathrm{BXRb}$-xenopus

PXR zebrafish

PXR opossum

PXR_COW

PXR chimpanzee

VDR/PXRa_ciona_(NP_001071847)

$\mathrm{VDR} / \mathrm{PXRb}$ ciona (NP-001037831)

VDR/PXRa_ciona_(KC5 61370)

VDR/PXRa_botrullus_(KC561372)

CAR_human

CAR_mouse

CAR_rat

CAR_rhesus

CAR_chimpanzee

CAR_COW

CAR_opossum

CAR_fur_seal

CAR_baik̄al_seal

CAR_dog

CAR_pig

ECR_tick

ECR silkworm

ECR/FXR sea urchin
110

120

130

140

150

160

170

180

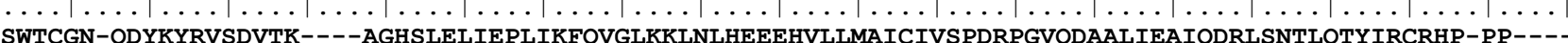
SWDCGS-QDYKYDITDVSR----AGHTLELIEPLIKFQVGLKKLNLHEEEHVLLMAICIVSPDRPGVQDAKLVEAIQDRLSNTLQTYIRCRHP-PP--SWTCGN-QDYKYRVSDVTK----AGHNLELIEPLIKFQVGLKKLNLHEEEHVLLMAICIVSPDRPGVQDAALIEA IQDRLSNTLQTYIRCRHP-PP--SWTCGN-QDYKYRVSDVTK----AGHSLELIEPLIKFQVGLKKLNLHEEEHVLLMAICIVSPDRPGVQDAALIEAIQDRLSNTLQTYIRCRHP-PP--SWTCGS-PDYKYQVSDVTR----AGHSLELIEPLIKFQVGLKKLNLHEEEHVLLMAICIVSPDRPGVQDAALVEAIQDRLSNTLQTYIRCRHP-PP---SWDCGS-QDYKYDVTDVSK----AGHTLELIEPLIKFQVGLKKLNLHEEEHVLLMAICIVSPDRPGVQDAKLVEAIQDRLSNTLQTYIRCRHP-PP---SWTCGS-EDFKYKVDDVTQ----AGHNMELLEPLVKFQVGLKKLDLHEEEHVLLMAICILSPDRPGLQDKALVES IQDRLSSTLQTYILCKHP-PP---SWSCGG-PDFKYCINDVTK----AGHTLDLLEPLVKFQVGLKKLNLHEEEHVLLMAICLLSPDRPGVQDHGRVEQLQDHLSETLQAYIQVNHP-----SWSCGG-PDFKYCVNDVTK----AGHTLELLEPLVKFQVGLKKLNLHEEEHVLLMAICLLSPDRPGVQDHARIEQLQDRLSEALQAYIRVNHP-----SWSCGG-PDFKYCINDVTK----AGHTLELLEPLVKFQVGLKKLKLHEEEHVLLMAICLLSPDRPGVQDHVRIEALQDRLCDVLQAYIRIQHP-----SWTCGS-NEFKYQIGDVMQ----AGHKLELLEPLVKFQVNMKKLDLHEAEHVLLMAICLFSPDRPGVQDRCRVEEVQEHLTETLRAYIACRHP-LS---SWTCGS-NDFKYKVSDVTO----AGHSMDLLEPLVKFOVGLKKLNLHEEEHVLLMAICILSPDRPGVODT SLVES IODRLSDTLOTY IRCRHP-PP--SWSCGG-PDFKYCINDVTK----AGHTLEILEPLVKFOVGLKKLNLHEEEHVLLMGICLLSPDRPGVODHARVEOLODRLPEALOAYIRINHPSWSCGGGPDFKYCVNDVTK- - AGHTIDI TWECRR TWECRR TWECGR TWECGR---LAYCFED-PN----GGFQKLLLDPLMKFHCMLKKLQLREEEYVLMQAISLFSPDR GVQRSVVDLLERFALTLKAYIECSRP-YP--IWECR - L Y AWECGQ---HCFTIKDGAL----AGFQQIYLEPLLKFHISLKKLRLHEAEYVLLVAMLLFSPDHASVTQRDF IDQLQEKVALTLKSYIDHRHP-MP--TWECGR---LSYCLED-PA----GGFQQLLLEPMLKFHYMLKKLQLHEEEYVLMQAISLFSPDRPGVVQHRVVDQLQEQYAITLKSYIECNRP-QP--QWKCGS---INYCIHDAFR----AGFQPFLLDPLFKFHHTLRKLGLGEEEYALIQALSLFSPDRPGVQEHQVIDKIHEKMALALKTWIDCRRT-GP--SWECGP---ITYNTEDMTM----AGFRQLFLEPLLRMHRMMRKLNLHNEEYALMAAMALFASDRPGVQDCKKIQNLQEHIALMLKRYIECQRPLSP--TWECGP---FTYDTEDMFL----AGFRQLFLEPLVRIHRMMRKLNLQSEEYAMMAALSIFASDRPGVCDWEKIQKLQEHIALTLKDFIDSQRPPSL--TWECGA---FTYNADDMTM----AGFSQQFLEPLLRIHCMMTKLNLESEAYALMATMALFSSDRPGVSDCEKIQNLQEHIALMLKAFIESHRPPSP---IWECGP---LQYCMDDAFR----AGFQHHLLDPMMNFHYTLRKLRLHEEEYVLMQALSLFSPDRPGVTDHKVIDRNQETLALTLKTY IEAKRN-GP---SWECGR---LSYYLD--PE----GALQQLLLEPVLKFHYMLKKLQLHSEEYVLMQAISLFSPDRPGVVQRRVVGQLQERFTVALKAY IECKRP-QP---TWECGR---LSYCVED-PA----GGFQQLLLEPVLKFHYMLKKLQLHKEEYVLMQAISLFSPDRPGVVQRLVVDQLQERFAMTLKAYIEFNRP-QP---TWECGR---LSYCLED-TA----GGFQQLLLEPMLKFHYMLKKLQLHEEEYVLMQAISLFSPDRPGVVQHRVVDQLQEKFAITLKSYIECNRP-QP---ALTLGP--DILYTRDSFLQG----GMSVEYTDNYLKFHEDLSALQLDDVEMSSLSAIALFSADRADLVDQQRVENQQEALALCLQAYSESSWK------KYMSDK---FQYKPSDFLQ----AGGNKEFVEKYNSLHIRMRKMKLQVEEICLLLALVLFSPDRPGLEDQAKVEQMQDCVANTLQAYEYTHKPPNESSFI ALTLGP--DIIYTRDSFIOG----GMSVEYTDNYLKFHEDLSALOLDDVEMSSLSAIALFSADRADLVDOORVENOOEALALCLOAYSESSWK--KYMSDK---FOYKPSDFL O----AGGNKFFVEKYNSLHIRMRKMKLOVEEICLLLALVLFSPDRPGLEDOAKVEOMODCVANTLOAYEYTHKPPNEALMCGPL NFLCGP - LRYTIEDGARW NFP NCO NFLCGP---LRYT IEDGAR----VGFQVEFLELLFHFHGTLRKLQLQEPEYVLLAAMALF SPDRPGTQRDE IDQLQEEMALTLQSY IKGQQR-RP---NFLCGP---LRYTIEDAAQ----AGFQEEFLEFLFGFHRTLRQLQLQEPEYVLMAAMALF SPDRPGITCREEIDQLQEEMALTLQNY IQGQQP-RP-TFLCGP---LRYTFQDGAH----VGFQEHFLELLLRFHMTLRRLKLQEPEYVLMAALALFSPDRPGVTQREQIDQFQEEMALTLQNYIRSQQA-RP---NFLCGP---LCYALEDGVH----VGFQEEFLELLFRFHATLRRLQLQEPEYVLMAAMALF SPDRPGVTRREEIDRLQEVTALTLQSYIKGQPP-RP--NFLCGP---LRYTLEDGVH----VGFQEEFLELLFRFHATLRRFQLQEPEYVLMAAMALFSPDRPGVTQKEEIDRLQEMMALTLQSYIKGQPP-RH---HFLCGP---LRYTMEDGVHGLSPAGFQEEFLELLFRFHGTLKRLQLQEPEYVLLAAMALF SPDRPGTRREE IDHLQEVMALTLQSYIRGQQP-RP---KFLCGP---LRYTIEDGAH----VGFQEEFLELLFGFHKTLRRLQLQEPEYVLMVAVALFSPDRPGVTQRKE IDQLQEEMALTLQSYIKGQQP-SL---SIVFAN--NQPYTRDNYRS-----ASVGDSADALFRFCRKMCQLRVDNAEYALLTAIVIFS-ERPSLVDPHKVERIQEYYIETLRMYSENHRPPGK---SVLFAN--NKAYTRDNYRQ-----GGMAYVIEDLLHFCRCMFAMGMDNVHFALLTAIVIFS-DRPGLEQPSLVEE IQRYYLNTLRIYI INQNSASSR--AIMFGN--EMPYTRKOLLE-----GGIGDLVDPMYNFAKSMSELDLDYAEF ILLMAIT ILSPDRPAINERERVEOMOETYLDMLRSYLKLRRPHEV---- 
VDR_human

VDR_mouse

VDR_rhesus

VDR_chimpanzee

VDR_COW

VDR_rat

VDR_xenopus laevis

VDR stickleback

VDR_medaka

VDR_zebrafish

VDR_lamprey

VDR quail

VDR_halibut

VDR_halibut

VDR_salmo

PXR_human

PXR_mouse

PXR_rat

PXR_rabbit

PXR_chicken

PXR_rhesus

PXR_medaka

PXR_xenopus_tropicalis

BXRā_xenopus

BXRb xenopus

PXR zebrafish

PXR opossum

PXR_cow

PXR chimpanzee

VDR/PXRa_ciona_(NP_001071847)

$\mathrm{VDR} / \mathrm{PXRb}$ ciona (NP-001037831)

VDR/PXRa_ciona_(KC5 61370$)$

VDR/PXRb ciona (KC561371)

VDR/PXRa botrullus (KC561372)

CAR_human

CAR_mouse

CAR_rat

CAR_rhesus

CAR_chimpanzee

CAR_COW

CAR_opossum

CAR_fur_seal

CAR_baik̄al_seal

CAR_dog

CAR_pig

ECR_tick

ECR silkworm

ECR/FXR sea urchin
210

220

230

240

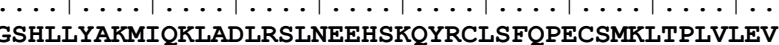

STH

GSHQYAKMIQKLADLRSLNEEHSKQYRSLSFQPENSMKLTPLVLEV GSHLLYAKMIQKL_ADLRSLNEEHSKQYRCLSFQPECSMKLTPLVLEV

GSHLLYAKMIGSHQL YAKMIQKLADDLRSLNEEHSKQYRSLSFQPENSMKLTPLVLEV GSRLLYAKMIQKLADLRSLNEEHSKQYRS ISFLPEHSMKLTPLMLEV GGRLLYAKMIQKLADLRSLNEEHSKQYRSLSFQPEHSMQLTPLVLEV GGRLLYAKMIQKLADLRSLNEEHSKQYRSLSFQPEHSMQLTPLVLEV GGRLLYAKMIQKLADLRSLNEEHSKQYRSLSFQPEHSMQLTPLVLEV CKHML YTKMVEKLTELRSLNEEHSKQYLQISQDAVNKEDLPPLLLEV GSRLIYAKMIOKLADLRSLNEEHSKOYRCLSFQPEHSMOLTPLVIEV GGRLIYAKMIOKLADLRSLNEEHSKOYRSLSFOPEHSMOLTPIVI VV GGRLL YARMIOKL ADLRSLNEEHSKOYRSISFOPEHSMOLI GR AHRFLFLKIMAMLTELRSINAQH TRLLRIQDIHP---FATPLMQEL AHR L FKIA AHRFLFLKIMAVIELRSINAQQ QQLLRIDDTHP---FATPLMQEL TMR L LKIMAVLTELRT INAQHQRLLRIQDTHP- - FATPLMREL EGRFLYAKLLLLLTELQTLKMENTRQILHIQDLSS----MTPLLSEI AHRFLFLKIMAMLTELRSINAQHQRLLRIQDIHP-- FATPLMQEL GKHLLY PKI IACLTELRSMSEEHSKQILQ IQDIQPD--TITPLLMEV QNRLLYPKIMECLTELRTVNDIHSKQLLEIWDIQP---DATPLLREV QNRLLYPKIMECLTELRTVNDIHSKQLLEIWDIQP---DATPLMREV QNRLLYPKIMECLTELRT IND IHSKQLMEIWDIQP---DVTPLMREV EKHLLFPKIMGCLTEMRSMNEEYTKQVLKIQDMQP---EVSPLWLEI AHRFLFLKIIAILTELRTINAOHTKRLLOIEDIHP---FATPLMREL AHRFLFLKIMAILTELRSLSAEHTQQLLRIHDVHP---FATPLMQEL AHRFLFLKIMAMLTELRS INAQHTQRLIRIQDIHP---FATPLMQEL -VRNRFAI IMSFLPRLRTLNSLCTTAFSQVK-KQFGE-EIRPLVKEV OARTMYCELLLILPILRT INMLFAONIMSLKQTNEK--DMNPI ILFV -IRNRFAI IMSFLPRLRTLNSLCTTAFSOVK-KOFGETRYCELLIILPILRTIN-

-SRVRFPKIMAYLTRIRTINWHISKTLDRIOSTSEAN-DIKPI-RDRFLYAKLIGLIAETRS INEAYGYOIOHIOGISA----MMPIIOEI TYRT QSR QSR IYAKLMLIALRSINAYSYEIHRIQGLSA-- MMLLGEI RDR IYAKLLGLIALLRSINEAYGQIQHIQGLSA-O-MMPLLEI RDRFLAKLLGLLAELRS INAYGYQIQH QGLSA----MMPLLQEI RDRFLYAKLLGLLADLRSIHDAFWQI RN QGLSI--MMPLLQEI QGRFLYAKMLGLLAELRSLSTEYGRQLQRIQELSA----LMPLLQEI RDRFLYAKLLGLLAELRSIDNAYGYQIQH IQGLSA----MMPLLQEI RDRFLYAKLLGLLAELRSINNAYGYQIQHIQGLSA----MMPLLQEI RDRFLYAKLLGLLAELRS INNAYGHQIQHIQGLSA----MMPLLQEI RDRFLYAKLLGLIAELRSINKEYWYQIONIOGLST---MMPLLOEI ---NYFARLLSILTELRTLGNMNAEMCFSLKVONK---KLPPFLAEI -CAVIYGRILSVLTEIRTLGTONSNMCISLKLKNR---KLPPFIEEI ---LLLPKVLMKLTELRSLNNSHSELLFOLKVKDQ---KI PPLLOEI 
Appendix 3 Alignment of predicted DNA-binding domains (DBDs) and ligand-binding domains (LBDs) from pregnane $X$ receptor (PXR)-related proteins from vertebrates, invertebrate chordates and protostome taxa. GenBank accession numbers are as follows: human (Homo sapiens) VDR [NM 00376], rhesus monkey (Macaca mulatta) VDR [Ensembl:

ENSMMUT00000009414], cow (Bos primigenius) VDR [Ensembl: ENSBTAT00000021832], mouse (Mus musculus) VDR [NM 008504], rat (Rattus norvegicus) VDR [NM 009504], Xenopus laevis VDR [U91849], zebrafish (Danio rerio) VDR [AF164512], medaka (Oryzias latipes) VDR [Ensembl: ENSORLT00000001311], stickleback fish (Gastrosteus aculeatus) VDR [Ensembl: ENSGACT00000006308], sea lamprey (Petromyzon marinus) VDR [AY249863], quail (Coturnix japonica) VDR [AAA56725], halibut (Paralichthys olivaceus) VDR [BAA95016], salmon (Salmo salar) VDR [CAG47089], Ciona intestinalis VDR/PXRa [NP 001071847 and KC561370], Ciona intestinalis VDR/PXRß [NP 001037831 and KC561371], Botryllus schlosseri VDR/PXRa [KC561372], human PXR [AF061056], rhesus monkey PXR [AF454671], mouse PXR [NP 035066], rat PXR [AF151377], rabbit (Oryctolagus cuniculus) PXR [AF31165], chicken (Gallus gallus) PXR [AF276753], Xenopus laevis BXRa [BC041187], Xenopus laevis BXRß [AF305201], Xenopus tropicalis PXR [Ensembl: ENSXETT00000039109], medaka PXR [Ensembl: ENSORLT00000022473], zebrafish PXR [AF454674], opossum PXR [Ensembl: ENSMODT00000023109], cow (Bos taurus) PXR [Ensembl: ENSBTAT00000026059], chimpanzee (Pan troglodytes) PXR [Ensembl:

ENSPTRT00000028510], human CAR [NP 005113], mouse CAR [NP 033933], rat CAR [NP_075230], rhesus monkey CAR [AAM76230], chimpanzee CAR [NP 001129087], cow CAR [NP 001073236], opossum CAR [Ensembl:

ENSMODT00000006393], fur seal (Callorhinus ursinus) CAR [BAD00039], Baikal seal (Phoca sibirica) CAR [BAD00038], dog (Canis sp.) CAR [Ensembl: ENSCAFT00000020528], pig (Sus scrofa) CAR [BAE54304], domestic silkworm (Bombyx mori) EcR [AAA87341], tick (Amblyomma americanum) EcR [AAB94566] and sea urchin (Strongylocentrotus purpuratus) EcR/FXR [NP 001123279]. Alignment was modified from Ekins et al. (2008). 


\section{APPENDIX FOUR}

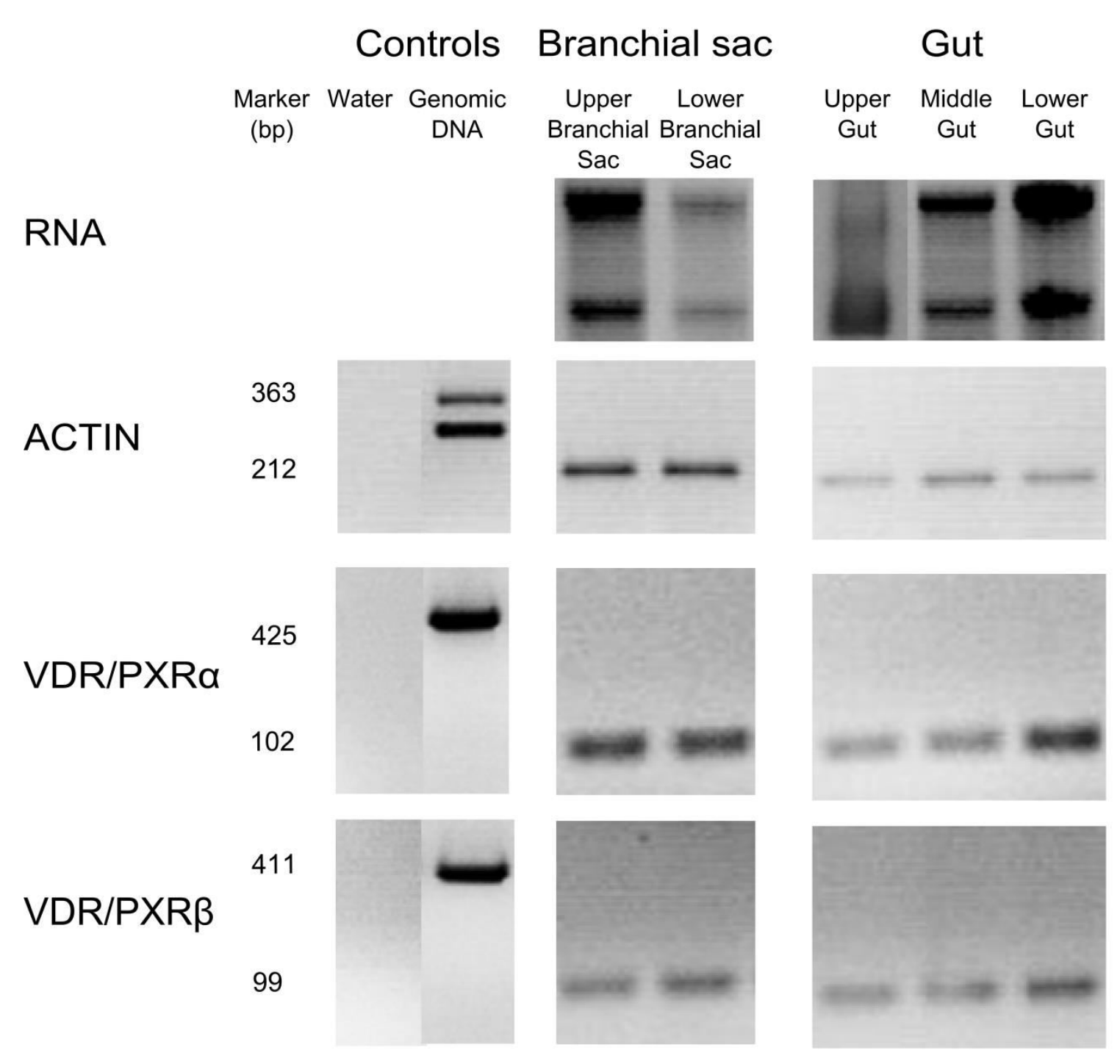

Appendix 4 Amplification of Ciona intestinalis vitamin $D$ receptor/pregnane $X$ receptor $\alpha(V D R / P X R \alpha), V D R / P X R \beta$ and actin partial coding sequences in five tissues using end-point polymerase chain reaction (PCR). Primers used for amplification are listed in Appendix 1B. Abbreviations: bp, base pair. 


\section{APPENDIX FIVE}

Appendix 5 Approximate probabilities $(p)$ of Cochran's C Test (A) and Tukey HSD Post Hoc Test (B) for the expression of Ciona intestinalis vitamin D receptor/pregnane $X$ receptor a (CNDR/PXRa), C. intestinalis VDR/PXR $\beta$ (CNDR/PXR $\beta$ ) and $C$. intestinalis actin (Ciactin) in various gut tissues. (A) Homogeneous data (non-significant Cochran $\mathrm{C}$ ) is shown in black numbers and non-homogeneous data (significant Cochran $\mathrm{C}$ ) is highlighted in red numbers. (B) P-values with $p<0.01$ were considered statistically significant (red numbers).

\begin{tabular}{|c|c|c|c|c|c|}
\hline $\mathbf{A}$ & $\begin{array}{l}\text { Hartley } \\
\text { F-max }\end{array}$ & $\begin{array}{l}\text { Cochran } \\
\text { C }\end{array}$ & $\begin{array}{l}\text { Bartlett } \\
\text { Chi-Sq. }\end{array}$ & df & $\mathbf{p}$ \\
\hline CNDR/PXRa & 21.62 & 0.63 & 5.14 & 4 & 0.273 \\
\hline$C N D R / P X R \beta$ & 1312 & 0.91 & 21.8 & 4 & 0.001 \\
\hline Ciactin & 35.36 & 0.51 & 5.08 & 4 & 0.278 \\
\hline
\end{tabular}

\begin{tabular}{llllll}
\hline B $\quad$ CNDR/PXRa & $\begin{array}{l}\text { Upper } \\
\text { Branchial } \\
\text { Sac }\end{array}$ & $\begin{array}{l}\text { Lower } \\
\text { Branchial } \\
\text { Sac }\end{array}$ & $\begin{array}{l}\text { Upper } \\
\text { Gut }\end{array}$ & $\begin{array}{l}\text { Middle } \\
\text { Gut }\end{array}$ & $\begin{array}{l}\text { Lower } \\
\text { Gut }\end{array}$ \\
\hline Upper Branchial Sac & & & & & \\
Lower Branchial Sac & 0.42 & & & & \\
Upper Gut & 0.01 & 0.01 & & & \\
Middle Gut & 0.01 & 0.01 & 0.01 & & \\
Lower Gut & 0.01 & 0.01 & 0.01 & 0.01 & \\
\hline
\end{tabular}

\section{CINDR/PXR $\beta$}

\begin{tabular}{|c|c|c|c|c|}
\hline \multicolumn{5}{|l|}{ Upper Branchial Sac } \\
\hline Lower Branchial Sac & 0.92 & & & \\
\hline Upper Gut & 0.37 & 0.80 & & \\
\hline Middle Gut & 0.02 & 0.07 & 0.37 & \\
\hline Lower Gut & 0.01 & 0.00 & 0.01 & 0.01 \\
\hline \multicolumn{5}{|l|}{ Ciactin } \\
\hline \multicolumn{5}{|l|}{ Upper Branchial Sac } \\
\hline Lower Branchial Sac & 1.00 & & & \\
\hline Upper Gut & 0.01 & 0.01 & & \\
\hline Middle Gut & 0.01 & 0.01 & 0.01 & \\
\hline Lower Gut & 0.01 & 0.01 & 0.01 & 0.01 \\
\hline
\end{tabular}




\section{APPENDIX SIX}

Appendix 6 Illumina ${ }^{\mathrm{TM}}$ sequencing read mapping statistics for Ciona intestinalis vitamin D receptor/pregnane $X$ receptor a (CNDR/PXRa, KC561370), $C$. intestinalis VDR/PXR (CNDR/PXR,$\underline{\mathrm{KC561371}}$ ) and Botryllus schlosseri VDR/PXRa (BsVDR/PXRa, KC561372).

\begin{tabular}{|c|c|c|c|}
\hline & \multicolumn{2}{|c|}{ Reads mapping to reference sequences } & \multirow[b]{2}{*}{ Single end read 2} \\
\hline & Pair end & Single end read 1 & \\
\hline CNDR/PXR $\alpha$ & $2,795,419(36 \%)$ & $1,380,943(35 \%)$ & $1,376,933(35 \%)$ \\
\hline CNDR/PXR $\beta$ & $1,923,917(24 \%)$ & $944,154(24 \%)$ & $940,214(24 \%)$ \\
\hline$B s \vee D R / P X R a$ & $2,892,225(37 \%)$ & $1,334,773(35 \%)$ & $1,363,318(34 \%)$ \\
\hline Unmapped & $262,741(3 \%)$ & $277,283(6 \%)$ & $256,688(7 \%)$ \\
\hline Total & $7,874,302$ & $3,937,153$ & $3,937,153$ \\
\hline
\end{tabular}




\section{APPENDIX SEVEN}

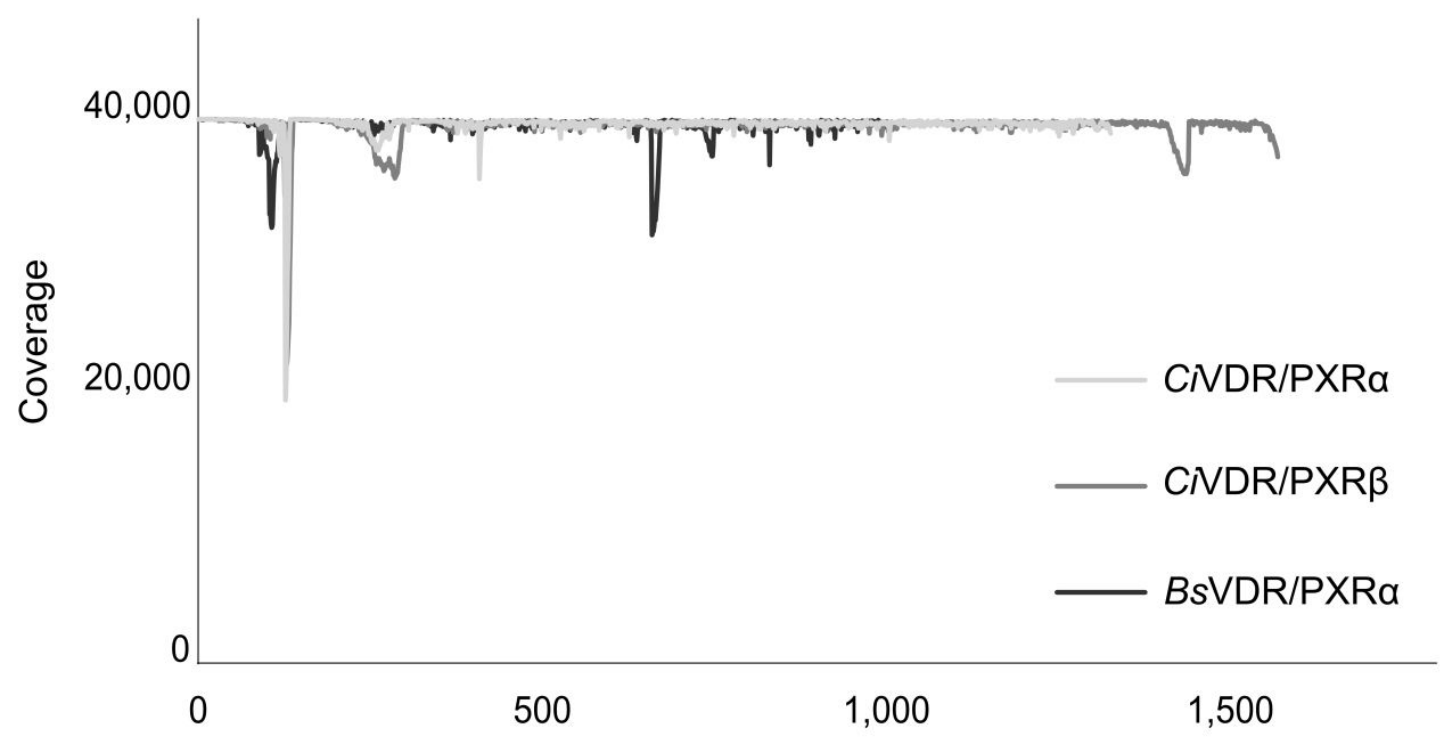

Nucleotide position in reference sequence in base pairs

Appendix 7 Coverage by position for Ciona intestinalis vitamin $D$ receptor/pregnane $\mathrm{X}$ receptor a (CNDR/PXRa, KC561370), C. intestinalis VDR/PXR $\beta$ (CNDR/PXR $\beta, \underline{\text { KC561371) }}$ and Botryllus schlosseri VDR/PXRa (BsVDR/PXRa, KC561372) using a maximum coverage of 40,000. 


\section{APPENDIX EIGHT}

Appendix 8 Nucleotide polymorphisms detected in Ciona intestinalis vitamin D receptor/pregnane $X$ receptor a (CNDR/PXRa, 1326 base pairs, bp), $C$. intestinalis VDR/PXRß (CNDR/PXRß, 1569 bp) and Botryllus schlosseri VDR/PXRa (BsVDR/PXRa, 1006 bp) using Illumina ${ }^{\text {TM }}$ sequencing. Number of single nucleotide polymorphisms (SNPs) and indels are given for various minimum variant frequencies $\left(f_{\min } \mathrm{var}\right)$. Shaded area indicates the minimum variant frequency 'cut-off' value (0.02) used in subsequent analyses.

\begin{tabular}{|c|c|c|c|c|c|c|c|c|c|c|c|}
\hline \multirow[b]{2}{*}{$f_{\min \text { var }}$} & \multicolumn{3}{|c|}{ CNDR/PXR $\alpha$} & \multicolumn{3}{|c|}{ CIVDR/PXR $\beta$} & \multicolumn{3}{|c|}{ BsVDR/PXRa } & \multicolumn{2}{|l|}{ Total } \\
\hline & $T_{\mathrm{SNPS}}$ & $f_{\text {SNPs }}$ & $T_{\text {indel }}$ & $T_{\text {SNPs }}$ & $f_{\mathrm{SNPs}}$ & $T_{\text {indel }}$ & $\mathrm{T}_{\mathrm{SNPS}}$ & $\mathbf{f}_{\mathrm{SNPs}}$ & $\mathbf{T}_{\text {indel }}$ & $\mathrm{T}_{\mathrm{SNPS}}$ & $\mathbf{T}_{\text {indel }}$ \\
\hline 0.01 & 28 & 2.11 & 2 & 53 & 3.38 & 5 & 42 & 4.17 & 1 & 42 & 3 \\
\hline 0.02 & 28 & 2.11 & 1 & 44 & 2.81 & 1 & 39 & 3.88 & & 38 & 1 \\
\hline 0.03 & 26 & 1.96 & 1 & 43 & 2.74 & & 38 & 3.78 & & 36 & 1 \\
\hline 0.04 & 26 & 1.96 & 1 & 40 & 2.55 & & 37 & 3.68 & & 35 & 1 \\
\hline 0.05 & 25 & 1.88 & 1 & 39 & 2.48 & & 37 & 3.68 & & 34 & 1 \\
\hline 0.06 & 25 & 1.88 & 1 & 38 & 2.55 & & 37 & 3.68 & & 34 & 1 \\
\hline 0.07 & 21 & 1.58 & 1 & 36 & 2.42 & & 37 & 3.68 & & 32 & 1 \\
\hline 0.08 & 19 & 1.43 & 1 & 34 & 2.29 & & 36 & 3.58 & & 30 & 1 \\
\hline 0.09 & 17 & 1.28 & & 32 & 2.17 & & 36 & 3.58 & & 29 & \\
\hline 0.1 & 16 & 1.21 & & 31 & 1.97 & & 35 & 3.48 & & 28 & \\
\hline 0.11 & 16 & 1.21 & & 30 & 1.91 & & 34 & 3.38 & & 27 & \\
\hline 0.12 & 16 & 1.21 & & 29 & 1.85 & & 34 & 3.38 & & 27 & \\
\hline 0.13 & 16 & 1.21 & & 29 & 1.85 & & 34 & 3.38 & & 27 & \\
\hline 0.14 & 15 & 1.13 & & 26 & 1.66 & & 34 & 3.38 & & 26 & \\
\hline 0.15 & 15 & 1.13 & & 26 & 1.66 & & 31 & 3.08 & & 25 & \\
\hline 0.16 & 15 & 1.13 & & 24 & 1.53 & & 31 & 3.08 & & 24 & \\
\hline 0.17 & 15 & 1.13 & & 24 & 1.53 & & 30 & 2.98 & & 24 & \\
\hline 0.18 & 15 & 1.13 & & 22 & 1.40 & & 29 & 2.88 & & 23 & \\
\hline 0.19 & 14 & 1.06 & & 19 & 1.21 & & 27 & 2.68 & & 21 & \\
\hline 0.2 & 14 & 1.06 & & 19 & 1.21 & & 27 & 2.68 & & 21 & \\
\hline
\end{tabular}




\section{APPENDIX NINE}

Appendix 9 Estimated transition/transversion rate ratios $(\mathrm{Ti} / \mathrm{TV})$ for Ciona intestinalis vitamin $\mathrm{D}$ receptor/pregnane $\mathrm{X}$ receptor a (CNDR/PXRa, 1326 base pairs, bp), C. intestinalis VDR/PXR $(C N D R / P X R \beta, 1569$ bp) and Botryllus schlosseri VDR/PXRa (BsVDR/PXRa, 1006 bp) using the Tamura-Nei model as implemented in the MEGA 5 software package.

\begin{tabular}{|c|c|c|c|c|c|c|c|c|c|}
\hline \multirow{2}{*}{$\begin{array}{l}\text { Substi- } \\
\text { tution }\end{array}$} & \multirow{2}{*}{$\begin{array}{l}\text { Counts } \\
\text { CivDR/PXRa }\end{array}$} & \multirow[b]{2}{*}{ CNDR/PXR $\beta$} & \multirow[b]{2}{*}{ BsVDR/PXRa } & \multicolumn{3}{|c|}{ Percent of total counts } & \multicolumn{3}{|c|}{ Rate of individual event } \\
\hline & & & & CINDR/PXRa & CIVDR/PXR $\beta$ & BsVDR/PXRa & CNDR/PXRa & CIVDR/PXR $\beta$ & BsVDR/PXRa \\
\hline \multicolumn{10}{|c|}{ Transitions } \\
\hline All & 22 & 31 & 31 & 78 & 71 & 80 & 0.017 & 0.022 & 0.032 \\
\hline$A->G$ & 9 & 14 & 17 & 32 & 32 & 44 & & & \\
\hline $\mathrm{T} \rightarrow \mathrm{C}$ & 13 & 17 & 14 & 46 & 39 & 36 & & & \\
\hline
\end{tabular}

\begin{tabular}{|c|c|c|c|c|c|c|c|c|c|}
\hline \multicolumn{10}{|c|}{ Transversions } \\
\hline All & 6 & 13 & 8 & 22 & 29 & 20 & 0.005 & 0.009 & 0.008 \\
\hline$A->T$ & 1 & 4 & 6 & 3.5 & 9 & 15 & & & \\
\hline$A \rightarrow C$ & 2 & 3 & 1 & 7.5 & 7 & 2.5 & & & \\
\hline $\mathrm{T} \rightarrow \mathrm{G}$ & 2 & 4 & 1 & 7.5 & 9 & 2.5 & & & \\
\hline$C \rightarrow \mathrm{G}$ & 1 & 2 & 0 & 3.5 & 4 & 0 & & & \\
\hline \multicolumn{7}{|c|}{$\mathrm{Ti} / \mathrm{T} v$ ratios } & 3.7 & 2.4 & 3.9 \\
\hline
\end{tabular}




\section{APPENDIX TEN}

Appendix 10 Nucleotide polymorphisms detected in Botryllus schlosseri vitamin D receptor/pregnane $X$ receptor a (BsVDR/PXRa, 1006 base pairs, bp) populations from Nelson Marina (New Zealand) and the eastern Mediterranean Coast (Michmoret Beach, Israel). One sequence from each population was selected as a reference sequence. Shaded areas indicate single nucleotide polymorphisms (SNPs) that were detected in a minimum of two sequences from independent amplification reactions.

\begin{tabular}{|c|c|c|c|c|c|c|c|}
\hline \multirow{2}{*}{$\begin{array}{l}\text { Position } \\
\text { (bp) }\end{array}$} & \multicolumn{3}{|c|}{ BsVDR/PXRa Nelson Marina } & \multirow{2}{*}{$\begin{array}{l}\text { Position } \\
\text { (bp) }\end{array}$} & \multicolumn{3}{|c|}{ BsVDR/PXRa Israel } \\
\hline & Ref & Var & aa subst. & & Ref & Var & aa subst. \\
\hline 9 & $\mathrm{~T}$ & C & $M->T$ & 8 & A & $G$ & $M->V$ \\
\hline 11 & $\mathrm{G}$ & A & $K \rightarrow E$ & 14 & A & $\mathrm{G}$ & $N \rightarrow D$ \\
\hline 32 & $\bar{T}$ & C & $C \rightarrow R$ & 31 & $T$ & A & \\
\hline 40 & $\mathrm{~T}$ & C & & 40 & C & $\mathrm{T}$ & \\
\hline 41 & A & $\mathrm{G}$ & $T \rightarrow A$ & 49 & $T$ & C & \\
\hline 59 & A & $\mathrm{G}$ & $\mathrm{T} \rightarrow \mathrm{A}$ & 57 & $\mathrm{~T}$ & C & $\mathrm{I}->\mathrm{T}$ \\
\hline 61 & $\mathrm{G}$ & A & & 60 & C & $\mathrm{T}$ & $T \rightarrow M$ \\
\hline 76 & A & $\mathrm{G}$ & & 61 & $\mathrm{G}$ & A & \\
\hline 80 & $\mathrm{~T}$ & C & $C \rightarrow R$ & 65 & A & G & $S \rightarrow G$ \\
\hline 82 & $\mathrm{~T}$ & C & & 76 & A & $\mathrm{G}$ & \\
\hline 86 & $\mathrm{G}$ & A & $A \rightarrow T$ & 82 & C & $\mathrm{T}$ & \\
\hline 94 & $\mathrm{G}$ & A & & 85 & A & $\mathrm{G}$ & \\
\hline 95 & $\mathrm{~T}$ & C & & 95 & C & $\mathrm{T}$ & \\
\hline 108 & T & C & $L \rightarrow P$ & 106 & C & $T$ & \\
\hline 115 & $\mathrm{~T}$ & C & & 115 & C & $\mathrm{T}$ & \\
\hline 130 & $\mathrm{G}$ & A & & 134 & A & G & $\mathrm{I}->\mathrm{V}$ \\
\hline 135 & C & $\mathrm{T}$ & $I->T$ & 144 & A & $\mathrm{G}$ & $H \rightarrow R$ \\
\hline 144 & $\mathrm{G}$ & A & $H \rightarrow R$ & 145 & C & $\mathrm{T}$ & \\
\hline 148 & $\bar{G}$ & A & & 148 & A & $G$ & \\
\hline 154 & $\mathrm{~T}$ & C & & 152 & A & $\mathrm{G}$ & I $->$ V \\
\hline 162 & A & $\mathrm{G}$ & $K \rightarrow R$ & 169 & $\mathrm{~T}$ & C & \\
\hline 181 & C & $\mathrm{T}$ & & 180 & A & $G$ & $N \rightarrow S$ \\
\hline 194 & C & $\mathrm{G}$ & $R \rightarrow G$ & 197 & A & $\mathrm{T}$ & $S \rightarrow C$ \\
\hline 197 & $\mathrm{~T}$ & A & $S \rightarrow C$ & 199 & $\mathrm{~T}$ & C & $S \rightarrow C$ \\
\hline 199 & $\mathrm{~T}$ & C & & 213 & A & $\mathrm{G}$ & $K \rightarrow R$ \\
\hline 209 & $T$ & C & $W \rightarrow R$ & 252 & C & $\widehat{T}$ & $T \rightarrow M$ \\
\hline 218 & $\mathrm{~T}$ & C & & 260 & $\mathrm{~T}$ & C & $S \rightarrow P$ \\
\hline 246 & $\mathrm{~T}$ & C & $L \rightarrow P$ & 271 & C & $\mathrm{T}$ & \\
\hline 252 & C & $\mathrm{T}$ & $\mathrm{T} \rightarrow \mathrm{M}$ & 281 & A & $\mathrm{G}$ & $\mathrm{N} \rightarrow \mathrm{D}$ \\
\hline 259 & C & $A$ & & 289 & $\mathrm{~T}$ & A & \\
\hline 271 & $\mathrm{~T}$ & C & & 303 & $A$ & $\mathrm{G}$ & $E \rightarrow G$ \\
\hline 281 & A & G & $N \rightarrow D$ & 305 & G & A & $E \rightarrow K$ \\
\hline 303 & A & $G$ & $E->G$ & 309 & A & $G$ & $D \rightarrow G$ \\
\hline 352 & $\mathrm{G}$ & A & & 333 & $\mathrm{G}$ & $\mathrm{T}$ & $R \rightarrow I$ \\
\hline 361 & A & G & & 349 & C & $\mathrm{T}$ & \\
\hline 405 & $\mathrm{~T}$ & $\mathrm{G}$ & $\mid->S$ & 361 & A & $\mathrm{G}$ & \\
\hline 421 & $\mathrm{G}$ & A & & 369 & $\mathrm{G}$ & A & $G \rightarrow E$ \\
\hline
\end{tabular}


Appendix 10 (cont.) Nucleotide polymorphisms detected in Botryllus schlosseri vitamin $\mathrm{D}$ receptor/pregnane $\mathrm{X}$ receptor $\alpha$ (BsVDR/PXRa, 1006 base pairs, bp) populations from Nelson Marina (New Zealand) and the eastern Mediterranean Coast (Michmoret Beach, Israel).

\begin{tabular}{|c|c|c|c|}
\hline \multirow{2}{*}{$\begin{array}{l}\text { Position } \\
\text { (bp) }\end{array}$} & \multicolumn{3}{|c|}{ BsVDR/PXRa Nelson Marina } \\
\hline & Ref & Var & (bp) \\
\hline 424 & $A$ & $\mathrm{G}$ & \\
\hline 456 & A & $\mathrm{G}$ & $\mathrm{H} \rightarrow \mathrm{R}$ \\
\hline 460 & $\mathrm{~T}$ & C & \\
\hline 489 & $A$ & $\mathrm{G}$ & $E \rightarrow G$ \\
\hline 517 & $\mathrm{~T}$ & C & \\
\hline 553 & G & A & \\
\hline 576 & A & G & $E \rightarrow G$ \\
\hline 580 & A & C & \\
\hline 607 & C & $\mathrm{T}$ & \\
\hline 625 & A & $\mathrm{T}$ & \\
\hline 643 & G & A & \\
\hline 658 & $\mathrm{G}$ & A & \\
\hline 661 & A & $\mathrm{T}$ & $D \rightarrow E$ \\
\hline 667 & $\mathrm{~T}$ & C & \\
\hline 686 & G & A & $E \rightarrow K$ \\
\hline 689 & $G$ & A & $E \rightarrow K$ \\
\hline 703 & C & $\mathrm{T}$ & \\
\hline 709 & G & A & \\
\hline 733 & $\mathrm{~T}$ & C & \\
\hline 734 & A & $\mathrm{G}$ & $M->V$ \\
\hline 742 & $\mathrm{~T}$ & $\mathrm{C}$ & \\
\hline 748 & A & $\mathrm{G}$ & \\
\hline 760 & $\mathrm{G}$ & $\mathrm{T}$ & \\
\hline 763 & C & $\mathrm{T}$ & \\
\hline 774 & C & $\mathrm{T}$ & $\mathrm{T}->\mathrm{I}$ \\
\hline 775 & A & G & \\
\hline 781 & $\mathrm{G}$ & A & \\
\hline 815 & A & $\mathrm{T}$ & $S \rightarrow T$ \\
\hline 822 & $\mathrm{~T}$ & C & $V \rightarrow A$ \\
\hline 865 & C & $\mathrm{T}$ & \\
\hline 896 & $\mathrm{~T}$ & C & $F \rightarrow L$ \\
\hline 903 & A & $\mathrm{G}$ & $K \rightarrow R$ \\
\hline 913 & $\mathrm{G}$ & A & \\
\hline 919 & $G$ & A & \\
\hline 945 & A & $\mathrm{G}$ & $\mathrm{H} \rightarrow \mathrm{R}$ \\
\hline 953 & A & G & $K \rightarrow E$ \\
\hline 954 & A & $\mathrm{G}$ & \\
\hline 999 & A & $\mathrm{G}$ & $K \rightarrow R$ \\
\hline 1000 & G & A & \\
\hline
\end{tabular}

\begin{tabular}{|c|c|c|c|}
\hline \multirow{2}{*}{$\begin{array}{l}\text { Position } \\
\text { (bp) }\end{array}$} & \multicolumn{3}{|c|}{ BsVDR/PXRa Israel } \\
\hline & Ref & Var & aa subst. \\
\hline 374 & $T$ & C & $S->P$ \\
\hline 387 & A & $\mathrm{G}$ & $N \rightarrow S$ \\
\hline 404 & A & $\mathrm{G}$ & \\
\hline 405 & T & C & $\mathrm{I}->\mathrm{V}$ or $\mathrm{T}$ \\
\hline 406 & C & $\mathrm{T}$ & \\
\hline 409 & A & G & \\
\hline 421 & G & A & \\
\hline 424 & $\mathrm{G}$ & A & \\
\hline 426 & C & $\mathrm{T}$ & $P \rightarrow L$ \\
\hline 443 & $\mathrm{~T}$ & C & $S \rightarrow P$ \\
\hline 447 & A & $\mathrm{G}$ & $H->R$ \\
\hline 465 & C & $\mathrm{T}$ & $A \rightarrow V$ \\
\hline 475 & G & A & \\
\hline 491 & $\mathrm{~T}$ & C & \\
\hline 536 & $\mathrm{~T}$ & C & \\
\hline 553 & G & A & \\
\hline 569 & $\mathrm{~T}$ & C & $C->R$ \\
\hline 573 & C & $T$ & $\mathrm{~T} \rightarrow \mathrm{M}$ \\
\hline 580 & C & A & \\
\hline 619 & G & A & \\
\hline 625 & $\mathrm{~T}$ & A & \\
\hline 627 & $\mathrm{~T}$ & C & $L->S$ \\
\hline 661 & T & A & $D \rightarrow E$ \\
\hline 667 & C & $T$ & \\
\hline 669 & $\mathrm{~T}$ & C & $\mathrm{I}->\mathrm{T}$ \\
\hline 673 & $A$ & $\mathrm{G}$ & \\
\hline 680 & A & $\mathrm{G}$ & $M->V$ \\
\hline 687 & A & $\mathrm{G}$ & $E \rightarrow G$ \\
\hline 703 & C & $\mathrm{T}$ & \\
\hline 715 & $\mathrm{~T}$ & C & \\
\hline 726 & T & C & $L->P$ \\
\hline 733 & T & C & \\
\hline 737 & C & T & \\
\hline 748 & G & A & \\
\hline 760 & $\mathrm{~T}$ & G & \\
\hline 763 & $\mathrm{~T}$ & C & \\
\hline 765 & $\mathrm{~T}$ & C & $M->T$ \\
\hline 772 & C & $T$ & \\
\hline 775 & G & A & \\
\hline 781 & G & A & \\
\hline 787 & $\mathrm{~T}$ & C & \\
\hline 800 & $\mathrm{G}$ & $-G$ & \\
\hline 819 & $\mathrm{~T}$ & C & $L \rightarrow P$ \\
\hline 826 & A & G & \\
\hline 835 & G & C & $Q \rightarrow H$ \\
\hline 837 & A & G & $E->G$ \\
\hline 853 & T & C & \\
\hline 865 & C & $\mathrm{T}$ & \\
\hline 896 & $\mathrm{~T}$ & C & $\mathrm{F} \rightarrow \mathrm{L}$ \\
\hline
\end{tabular}


Appendix 10 (cont.) Nucleotide polymorphisms detected in Botryllus schlosseri vitamin $\mathrm{D}$ receptor/pregnane $\mathrm{X}$ receptor $\alpha(B s \mathrm{VR} / \mathrm{PXR} \alpha, 1006$ base pairs, bp) populations from Nelson Marina (New Zealand) and the eastern Mediterranean Coast (Michmoret Beach, Israel).

\begin{tabular}{llll}
\hline Position & \multicolumn{3}{l}{ BsVDR/PXRa Nelson Marina } \\
(bp) & Ref & Var & (bp) \\
\hline
\end{tabular}

\begin{tabular}{llll}
\hline Position & \multicolumn{3}{l}{ BsVDR/PXRa Israel } \\
(bp) & Ref & Var & aa subst. \\
\hline 919 & G & A & \\
931 & A & G & \\
991 & C & T & \\
1000 & A & G & \\
\hline & & 89 & $42(47 \%)$ \\
& & 8.85 & 3.97 \\
\hline
\end{tabular}

\begin{tabular}{lll}
\hline $\mathbf{T}_{\text {SNPs }}$ & 76 & $34(44 \%)$ \\
$\mathbf{f}_{\text {SNPs }}$ & 7.55 & 3.38 \\
\hline
\end{tabular}

Abbreviations: aa subst., amino acid substitution caused by SNP in variant sequence; bp, base pair; $f_{S N P s}$, SNP frequency (SNP/100 bp); Ref, reference sequence; $T_{S N P s}$, total SNPs; Var, variant sequence. 


\section{APPENDIX ELEVEN}

Appendix 11 Activation of the tunicate yeast bioassays by four synthetic chemicals (A) and five algal toxins (B). Ligand-dependent induction of $\beta$-galactosidase enzymatic activity was measured in yeast strains carrying plasmids pGAL4.CILBD 431 .VP16, pGAL4.CILBD.VP16 or pGAL4.BSLBD.VP16. Mean effective concentrations (EC 50 values) and 95\% confidence intervals $(95 \% \mathrm{Cl})$ are given in $\mu \mathrm{M}$ for synthetic chemicals $(\mathbf{A})$ and in $\mathrm{nM}$ for algal toxins $(\mathbf{B})$. $95 \%$ confidence intervals represent variation within a triplicate measurement $(n=3)$. Coefficients of variance $(C V)$ are given in \% for triplicate intra-plate measurements. All compounds were dissolved in dimethyl sulfoxide (DMSO).

A

\begin{tabular}{|c|c|c|c|c|c|c|c|c|}
\hline \multirow[t]{2}{*}{ Chemical name } & \multirow[t]{2}{*}{ Toxin type } & \multirow{2}{*}{$\begin{array}{l}\text { Supplier } \\
\text { (Catalogue No.) }\end{array}$} & \multicolumn{2}{|c|}{ pGAL4.CiLBD $\Delta 31 . V P 16$} & \multicolumn{2}{|c|}{ pGAL4.CiLBD.VP16 } & \multicolumn{2}{|c|}{ pGAL4.BsLBD.VP16 } \\
\hline & & & $\mathrm{EC}_{50}(95 \% \mathrm{Cl})$ & CV & $\mathrm{EC}_{50}(95 \% \mathrm{Cl})$ & CV & $\mathrm{EC}_{50}(95 \% \mathrm{Cl})$ & CV \\
\hline $\begin{array}{l}N \text {-butyl-p-amino- } \\
\text { benzoate }^{\text {a }}\end{array}$ & $\begin{array}{l}\text { Local } \\
\text { anaesthetic }\end{array}$ & $\begin{array}{l}\text { Sigma-Aldrich } \\
\text { (B7753) }\end{array}$ & $9.4(7.8-11)$ & 25 & $6.1(4.9-7.5)$ & 10 & $2.4(0.5-12)$ & 28 \\
\hline Carbamazepine $^{\mathrm{b}}$ & Anti-depressant & $\begin{array}{l}\text { Sigma-Aldrich } \\
(\mathrm{C} 4024)\end{array}$ & $\mathrm{NI}(\mathrm{NI})$ & DNC & $\mathrm{NI}(\mathrm{NI})$ & DNC & $\mathrm{NI}(\mathrm{NI})$ & DNC \\
\hline$P$-aminobenzoic acid ${ }^{\mathrm{b}}$ & Supplement & $\begin{array}{l}\text { Sigma-Aldrich } \\
\text { (A9878) }\end{array}$ & $\mathrm{NI}(\mathrm{NI})$ & DNC & $\mathrm{NI}(\mathrm{NI})$ & DNC & $\mathrm{NI}(\mathrm{NI})$ & DNC \\
\hline Bisphenol-A (BPA) ${ }^{\mathrm{D}}$ & EDC & $\begin{array}{l}\text { Sigma-Aldrich } \\
\text { (B1760) }\end{array}$ & $76(67-85)$ & 11 & $66(59-73)$ & 17 & $58(48-69)$ & 16 \\
\hline
\end{tabular}


Appendix 11 (cont.) Activation of the tunicate yeast bioassays by four synthetic chemicals (A) and five algal toxins (B).

B

\begin{tabular}{|c|c|c|c|c|c|c|c|c|}
\hline \multirow[t]{2}{*}{ Chemical name } & \multirow[t]{2}{*}{ Toxin type } & \multirow{2}{*}{$\begin{array}{l}\text { Supplier } \\
\text { (Catalogue No.) }\end{array}$} & \multicolumn{2}{|c|}{ pGAL4.CiLBD $\triangle 31 . V P 16$} & \multicolumn{2}{|c|}{ pGAL4.CiLBD.VP16 } & \multicolumn{2}{|c|}{ pGAL4.BsLBD.VP16 } \\
\hline & & & $\mathrm{EC}_{50}(95 \% \mathrm{Cl})$ & CV & $\mathrm{EC}_{50}(95 \% \mathrm{Cl})$ & CV & $\mathrm{EC}_{50}(95 \% \mathrm{Cl})$ & CV \\
\hline Okadaic acid $^{\mathrm{b}}$ & $\begin{array}{l}\text { Microalgal } \\
\text { biotoxin }\end{array}$ & $\begin{array}{l}\text { Sapphire Bioscience } \\
\text { (AB120375) }\end{array}$ & $\mathrm{NI}(\mathrm{NI})$ & DNC & $\mathrm{NI}(\mathrm{NI})$ & DNC & $\mathrm{NI}(\mathrm{NI})$ & DNC \\
\hline Pectenotoxin- $11^{\mathrm{b}}$ & $\begin{array}{l}\text { Microalgal } \\
\text { biotoxin }\end{array}$ & $\begin{array}{l}\text { MacKenzie et al. } \\
2013\end{array}$ & $913(238-3506)$ & 154 & $545(150-1985))$ & 63 & $\mathrm{NI}(\mathrm{NI})$ & DNC \\
\hline Portimine ${ }^{b}$ & $\begin{array}{l}\text { Microalgal } \\
\text { biotoxin }\end{array}$ & Selwood et al. 2013 & $\mathrm{NI}(\mathrm{NI})$ & DNC & $\mathrm{NI}(\mathrm{NI})$ & DNC & $\mathrm{NI}(\mathrm{NI})$ & DNC \\
\hline Microcystin-RR ${ }^{b}$ & $\begin{array}{l}\text { Cyanobac-terial } \\
\text { toxin }\end{array}$ & $\begin{array}{l}\text { DHI Lab Products } \\
\text { (PPS-MCRR) }\end{array}$ & $\mathrm{NI}(\mathrm{NI})$ & DNC & $\mathrm{NI}(\mathrm{NI})$ & DNC & $\mathrm{NI}(\mathrm{NI})$ & DNC \\
\hline Anatoxin- $A^{b}$ & $\begin{array}{l}\text { Cyanobac-terial } \\
\text { toxin }\end{array}$ & $\begin{array}{l}\text { NRC Canada } \\
\text { (IMB-CRM-ATX) }\end{array}$ & $\mathrm{NI}(\mathrm{NI})$ & DNC & $\mathrm{NI}(\mathrm{NI})$ & DNC & $\mathrm{NI}(\mathrm{NI})$ & DNC \\
\hline
\end{tabular}

Abbreviations: BPA, bisphenol-A; DNC, did not compute; EDC, endocrine disrupting chemical; $\mathrm{NI}$, no induction of $\beta$-galactosidase enzymatic activity.

${ }^{a}$ Strains were incubated for 24 hours.

${ }^{\mathrm{b}}$ Strains were incubated for 48 hours. 


\section{APPENDIX TWELVE}

Appendix 12 Summary of the templates, primers and restriction enzymes used to generate the plasmids used in this study. Sites for restriction enzymes encoded within the primer sequences are underlined and regions annealing to target genes are indicated in grey. The base vector pGAL4 was obtained from Clontech (Clontech Laboratories Inc., Catalogue No. 630443).

\begin{tabular}{|c|c|c|c|c|c|}
\hline Plasmid & Template & Primers & Region amplified & Enzymes & Base vector \\
\hline pGAL4 & $\mathrm{N} / \mathrm{A}$ & $\mathrm{N} / \mathrm{A}$ & $\mathrm{N} / \mathrm{A}$ & $\mathrm{N} / \mathrm{A}$ & pGBKT7 \\
\hline pGAL4.VP16 & $\begin{array}{l}\text { pHCA/GAL4(1- } \\
\text { 93).ER.VP16 }{ }^{\text {a }}\end{array}$ & $\begin{array}{l}\text { forward: } \\
\text { 5'-CCGGATCCGTGAGCTCCACTTAGACGGCGAGGAC-3' } \\
\text { reverse: } \\
\text { 5'-CC. }\end{array}$ & $\begin{array}{l}103,274-103,480 \\
\text { of } \underline{\mathrm{HM} 585511}\end{array}$ & $\begin{array}{l}\text { BamHI/ } \\
\text { Pstl }\end{array}$ & pGBKT7 \\
\hline pGAL4.CiLBD & $\begin{array}{l}\text { pM2-GAL4- } \\
\text { CNDR/PXRa }^{\mathrm{b}}\end{array}$ & $\begin{array}{l}\text { forward: } \\
\text { 5'-CCCCATGGAGGGAATGAAGCGCGAATGTATCATGTCA-3' } \\
\text { reverse: } \\
\text { 5'-CCGGATCCCATTCAAGCGTTTTCCACGG-3' }\end{array}$ & $\begin{array}{l}860-1798 \text { of } \\
\text { NM } 001078379 \\
\end{array}$ & $\begin{array}{l}\text { Ncol/ } \\
\text { BamHI }\end{array}$ & pGBKT7 \\
\hline $\begin{array}{l}\text { pGAL4.CiLBD } \\
\text {.VP16 }\end{array}$ & $\begin{array}{l}\text { pM2-GAL4- } \\
\text { CNDR/PXRa }^{\mathrm{b}}\end{array}$ & $\begin{array}{l}\text { forward: } \\
\text { 5'-CCCCATGGAGGGAATGAAGCGCGAATGTATCATGTCA-3' } \\
\text { reverse: } \\
\text { 5'-CCGGATCCCAGCGTTTTCCACGGTGGGTTGAAC-3' }\end{array}$ & $\begin{array}{l}860-1795 \text { of } \\
\text { NM } 001078379 \\
\end{array}$ & $\begin{array}{l}\text { Ncol/ } \\
\text { BamHI }\end{array}$ & GAL4.VP16 \\
\hline
\end{tabular}


Appendix 12 (cont.) Summary of the templates, primers and restriction enzymes used to generate the plasmids used in this study.

\begin{tabular}{|c|c|c|c|c|c|}
\hline Plasmid & Template & Primers & Region amplified & Enzymes & Base vector \\
\hline $\begin{array}{l}\text { pGAL4.CiLBD } \\
\Delta 31 . \text { VP16 }\end{array}$ & $\begin{array}{l}\text { pM2-GAL4- } \\
\text { CNDR/PXRa }\end{array}$ & $\begin{array}{l}\text { forward: } \\
\text { 5'-CC.CCATGGAGGGAATGAAGCGCGAATGTATCATGTCA-3' } \\
\text { reverse: } \\
\text { 5'-CCEGATCCCACTGTTGAGGGTTCTCAGCCTTGG-3' }\end{array}$ & $\begin{array}{l}860-1702 \text { of } \\
\text { NM } 001078379 \\
\end{array}$ & $\begin{array}{l}\mathrm{Ncol} / \\
\text { BamHI }\end{array}$ & GAL4.VP16 \\
\hline $\begin{array}{l}\text { pGAL4.BsLBD } \\
\text {.VP16 }\end{array}$ & $\begin{array}{l}\text { Botryllus } \\
\text { schlosseri cDNA }\end{array}$ & $\begin{array}{l}\text { forward: } \\
\text { 5'-CC.CCATGGAGGGCATGAAGAAAGAGTGCATCATG-3' } \\
\text { reverse: } \\
\text { 5'-CCGGATCCCCACGTTGGGCATACATTCAAATAC-3' }\end{array}$ & $\begin{array}{l}116-1006^{\mathrm{C}} \text { of } \\
\mathrm{KC} 561372\end{array}$ & $\begin{array}{l}\text { Ncol/ } \\
\text { BamHI }\end{array}$ & GAL4.VP16 \\
\hline pGAL4.BsLBD & $\begin{array}{l}\text { Botryllus } \\
\text { schlosseri cDNA }\end{array}$ & $\begin{array}{l}\text { forward: } \\
\text { 5'-CC.CCATGGAGGGCATGAAGAAAGAGTGCATCATG-3' } \\
\text { reverse: } \\
\text { 5'-CCGGATCCTCATTACACGTTGGGCATACATTCAAATAC-3' }\end{array}$ & $\begin{array}{l}116-1006^{\mathrm{C}} \text { of } \\
\mathrm{KC} 561372\end{array}$ & $\begin{array}{l}\mathrm{Ncol} / \\
\text { BamHI }\end{array}$ & GAL4.VP16 \\
\hline
\end{tabular}

Abbreviations: N/A, not available.

${ }^{a}$ Plasmid pHCA/GAL4(1-93).ER.VP16 was generously provided by Prof Didier Picard, University of Geneva, Switzerland (Louvion et al. 1993).

${ }^{b}$ Plasmid pM2-GAL4-CIVDR/PXRa was generously provided by Prof Matthew Krasowski, University of lowa, U.S.A. (Reschly et al. 2007; Ekins et al. 2008; Fidler et al. 2012).

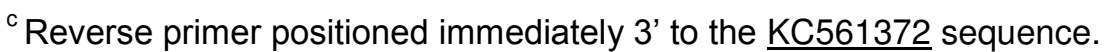




\section{APPENDIX THIRTEEN}

Appendix 13 Summary of the predicted proteins encoded by the seven plasmids used in this study. Stop codons are indicated $\left({ }^{*}\right)$.

\begin{tabular}{|c|c|c|c|c|}
\hline Plasmid name & $\begin{array}{l}\text { GAL4-DBD sequence } \\
\text { (147 residues) }\end{array}$ & $\begin{array}{l}\text { Linker } \\
\text { sequence }\end{array}$ & LBD sequence & $\begin{array}{l}\text { C-terminal/ } \\
\text { VP16 sequence }\end{array}$ \\
\hline pGAL4 & $\begin{array}{l}\text { MKLLSSIEQACDICRLKKLKCSKEK } \\
\text { PKCAKCLKNNWECRYSPKTKRSPL } \\
\text { TRAHLTEVESRLERLEQLFLLIFPRE } \\
\text { DLDMILKMDSLQDIKALLTGLFVQD } \\
\text { NVNKDAVTDRLASVETDMPLTLRQ } \\
\text { HRISATSSSEESSNKGQRQLTVS }\end{array}$ & $\begin{array}{l}\text { PEFVIRLTI } \\
\text { GRAAIMEE } \\
\text { QKLISEED } \\
\text { LHMAME }\end{array}$ & $\mathrm{N} / \mathrm{A}$ & $\begin{array}{l}\text { AEFPGIRRPAA } \\
\text { AA* }^{*}\end{array}$ \\
\hline pGAL4.VP16 & $\begin{array}{l}\text { MKLLSSIEQACDICRLKKLKCSKEK } \\
\text { PKCAKCLKNNWECRYSPKTKRSPL } \\
\text { TRAHLTEVESRLERLEQLFLLIFPRE } \\
\text { DLDMILKMDSLQDIKALLTGLFVQD } \\
\text { NVNKDAVTDRLASVETDMPLTLRQ } \\
\text { HRISATSSSEESSNKGQRQLTVS }\end{array}$ & $\begin{array}{l}\text { PEFVIRLTI } \\
\text { GRAAIMEE } \\
\text { QKLISEED } \\
\text { LHMAMEA } \\
\text { EFP }\end{array}$ & N/A & $\begin{array}{l}\text { GIRELHLDGED } \\
\text { VAMAHADALD } \\
\text { DFDLDMLGDG } \\
\text { DSPGPGFTPH } \\
\text { DSAPYGALDM } \\
\text { ADFEFEQMFT } \\
\text { DALGIDEYGG* }\end{array}$ \\
\hline pGAL4.CiLBD & $\begin{array}{l}\text { MKLLSSIEQACDICRLKKLKCSKEK } \\
\text { PKCAKCLKNNWECRYSPKTKRSPL } \\
\text { TRAHLTEVESRLERLEQLFLLIFPRE } \\
\text { DLDMILKMDSLQDIKALLTGLFVQD } \\
\text { NVNKDAVTDRLASVETDMPLTLRQ } \\
\text { HRISATSSSEESSNKGQRQLTVS }\end{array}$ & $\begin{array}{l}\text { PEFVIRLTI } \\
\text { GRAAIMEE } \\
\text { QKLISEED } \\
\text { LHMAME }\end{array}$ & $\begin{array}{l}\text { GMKRECIMSPDEIQMKKTLVLSNRIKRATMQWVPMELTN } \\
\text { DQKILLDTICSAFIQSNNLPEKGVIRGGEEVRQDADKGSP } \\
\text { CSSPSSSSNNSNFLELIKRIANDIAARTPSNMTNPAHVVHF } \\
\text { TDIMEFSIKEIIKFCKKIPTFMDLDLDQIALLKSGCTEILFIK } \\
\text { ANYTYDLEKKALTLGPDILYTRDSFLQGGMSVYTDNYLK } \\
\text { FHEDLSALQLDDVEMSSLSAIALFSADRADLVDQQRVEN } \\
\text { QQEALALCLQAYSESSWKVRNRFAIIMSFLPRLRTLNSLC } \\
\text { TTAFSQVKKQFGEEIRPLVKEVQPTVENA* }\end{array}$ & $\mathrm{N} / \mathrm{A}$ \\
\hline
\end{tabular}


Appendix 13 (cont.) Summary of the predicted proteins encoded by the seven plasmids used in this study.

\begin{tabular}{|c|c|c|c|c|}
\hline pGAL4.CiLBD.VP16 & $\begin{array}{l}\text { MKLLSSIEQACDICRLKKLKCSKE } \\
\text { KPKCAKCLKNNWECRYSPKTKRS } \\
\text { PLTRAHLTEVESRLERLEQLFLLIF } \\
\text { PREDLDMILKMDSLQDIKALLTGL } \\
\text { FVQDNVNKDAVTDRLASVETDMP } \\
\text { LTLRQHRISATSSSEESSNKGQR } \\
\text { QLTVS }\end{array}$ & $\begin{array}{l}\text { PEFVIR } \\
\text { LTIGRA } \\
\text { AIMEE } \\
\text { QKLISE } \\
\text { EDLHM } \\
\text { AME }\end{array}$ & $\begin{array}{l}\text { GMKRECIMSPDEIQMKKTLVLSNRIKRATMQWVPMELTND } \\
\text { QKILLDTICSAFIQSNNLPEKGVIRGGEEVRQDADKGSPCS } \\
\text { SPSSSSNNSNFLELIKRIANDIAARTPSNMTNPAHVVHFTDI } \\
\text { MEFSIKEIIKFCKKIPTFMDLDLKDQIALLKSGCTEILFIKANYT } \\
\text { YDLEKKALTLGPDILYTRDSFLQGGMSVYTDNLKFHEDL } \\
\text { SALQLDDVEMSSLSAIALFSADRADLVDQRVEQRQALA } \\
\text { LCLQAYSESSWKVRNRAIIMSFLPRLRTLNSLCTTAFSQV } \\
\text { KKQFGEEIRPLVKEVQPTVENA }\end{array}$ & $\begin{array}{l}\text { GIRELHLDGED } \\
\text { VAMAHADALD } \\
\text { DFDLDMLGDG } \\
\text { DSPGPGFTPH } \\
\text { DSAPYGALDM } \\
\text { ADFEFEQMFT } \\
\text { DALGIDEYGG* }\end{array}$ \\
\hline pGAL4.CiLBD $\triangle 31$.VP16 & $\begin{array}{l}\text { MKLLSSIEQACDICRLKKLKCSKE } \\
\text { KPKCAKCLKNNWECRYSPKTKRS } \\
\text { PLTRAHLTEVESRLERLEQLFLLIF } \\
\text { PREDLDMILKMDSLQDIKALLTGL } \\
\text { FVQDNVNKDAVTDRLASVETDMP } \\
\text { LTLRQHRISATSSSEESSNKGQR } \\
\text { QLTVS }\end{array}$ & $\begin{array}{l}\text { PEFVIR } \\
\text { LTIGRA } \\
\text { AIMEE } \\
\text { QKLISE } \\
\text { EDLHM } \\
\text { AME }\end{array}$ & $\begin{array}{l}\text { GMKRECIMSPDEIQMKKTLVLSNRIKRATMQWVPMELTND } \\
\text { QKILLDTICSAFIQSNNLPEKGVIRGGEEVRQDADKGSPCS } \\
\text { SPSSSSNNSNFLELIKRIANDIAARTPSNMTNPAHVVHFTDI } \\
\text { MEFSIKEIIKFCKKIPTFMDLDLKDQIALLKSGCTEILFIKANYT } \\
\text { YDLEKKALTLGPDILYTRDSFLQGGMSVEYTDNYLKFHEDL } \\
\text { SALQLDDVEMSSLSAIALFSADRADLVDQQRVENQQEALA } \\
\text { LCLQAYSESSWKVRNRFAIIMSFLPRLRTLNS }\end{array}$ & $\begin{array}{l}\text { GIRELHLDGED } \\
\text { VAMAHADALD } \\
\text { DFDLDMLGDG } \\
\text { DSPGPGFTPH } \\
\text { DSAPYGALDM } \\
\text { ADFEFEQMFT } \\
\text { DALGIDEYGG* }\end{array}$ \\
\hline pGAL4.BsLBD & $\begin{array}{l}\text { MKLLSSIEQACDICRLKKLKCSKE } \\
\text { KPKCAKCLKNNWECRYSPKTKRS } \\
\text { PLTRAHLTEVESRLERLEQLFLLIF } \\
\text { PREDLDMILKMDSLQDIKALLTGL } \\
\text { FVQDNVNKDAVTDRLASVETDMP } \\
\text { LTLRQHRISATSSSEESSNKGQR } \\
\text { QLTVS }\end{array}$ & $\begin{array}{l}\text { PEFVIR } \\
\text { LTIGRA } \\
\text { AIMEE } \\
\text { QKLISE } \\
\text { EDLHM } \\
\text { AME }\end{array}$ & $\begin{array}{l}\text { GMKKECIMSHTEIQMKKNLMLNNKIKRSVMEWKPLEYSAE } \\
\text { RRQLVTLISESHTQTNGPPDIREEDGMLTIRSRDPGASPAS } \\
\text { TSSGFSDLINQMTQDIVATAPGPSNPSKSHLRHFSAIMEFS } \\
\text { KEIIKFCKKIPSFTSLSLKDQITLLKSGCTEILFIKANYTYDRE } \\
\text { QNALMCGPGKYTRDSFILGGMSEEYDCYLQFHHDSH } \\
\text { MLLDESELACMCATSLFSGDRDGLENRSLVEEVQERITVAL } \\
\text { QSYTETIYHSRVRFPKIMAYLTRLRTLNWHISKTLDRIQSTS } \\
\text { EANDIKPLVFECMPNV** }\end{array}$ & N/A \\
\hline
\end{tabular}




\section{APPENDIX FOURTEEN}

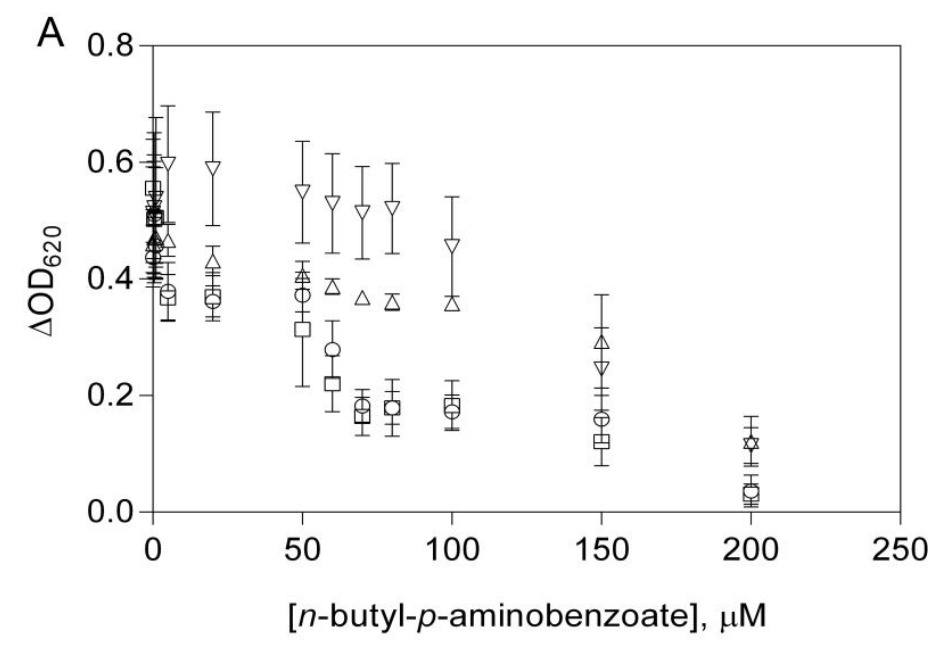

- pGAL4

- $\quad$ PGAL4.VP16

$\triangle \quad$ pGAL4.CiLBD.VP16

$\nabla \quad$ pGAL4.BsLBD.VP16

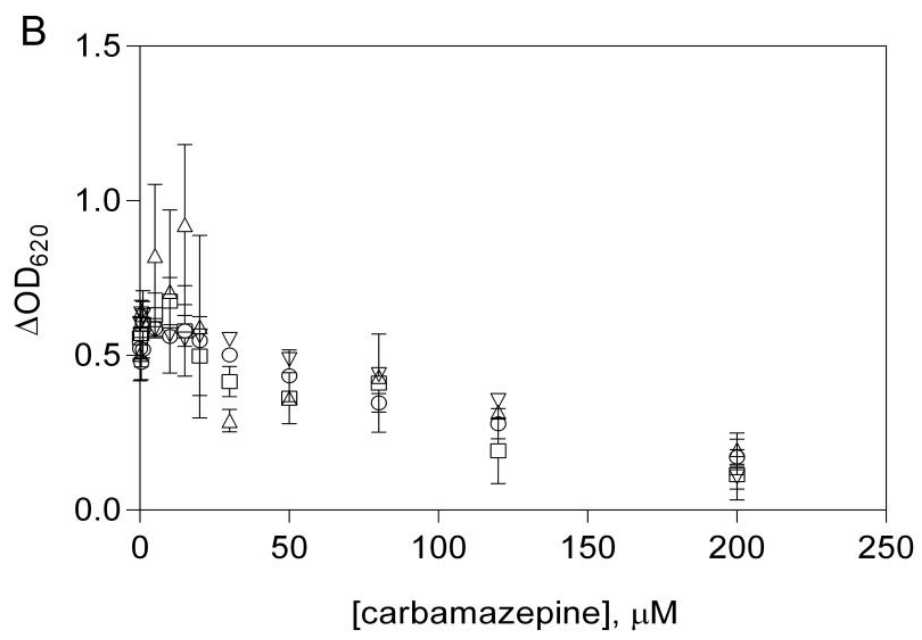

- $\quad$ GGAL4

口 $\quad$ pGAL4.VP16

$\triangle \quad$ PGAL4.CILBD

$\nabla \quad$ pGAL4.BSLBD

Appendix 14 Suppression of growth of recombinant yeast strains by: (A) $n$ butyl-p-aminobenzoate and (B) carbamazepine. Cell densities were quantified by $\mathrm{OD}_{620}$ values. Growth suppression (i.e. toxic) effects were detected by changes in $\mathrm{OD}_{620}$ values over time $\left(\Delta \mathrm{OD}_{620}=\mathrm{OD}_{620}\right.$ after $48 \mathrm{~h}-\mathrm{OD}_{620}$ after $16 \mathrm{~h})$. Data points represent means of $n=3$ replicates. Error bars show \pm one standard deviation. Strains carrying plasmids pGAL4.CILBD $\Delta 31 . V P 16$, pGAL4.CILBD.VP16 and pGAL4.BSLBD.VP16 were omitted from this analysis because the media colouration arising from hydrolysis of chlorophenol red- $\beta-D-$ galactopyranoside (CPRG) interfered with $\mathrm{OD}_{620}$ measurements. 


\section{APPENDIX FIFTEEN}

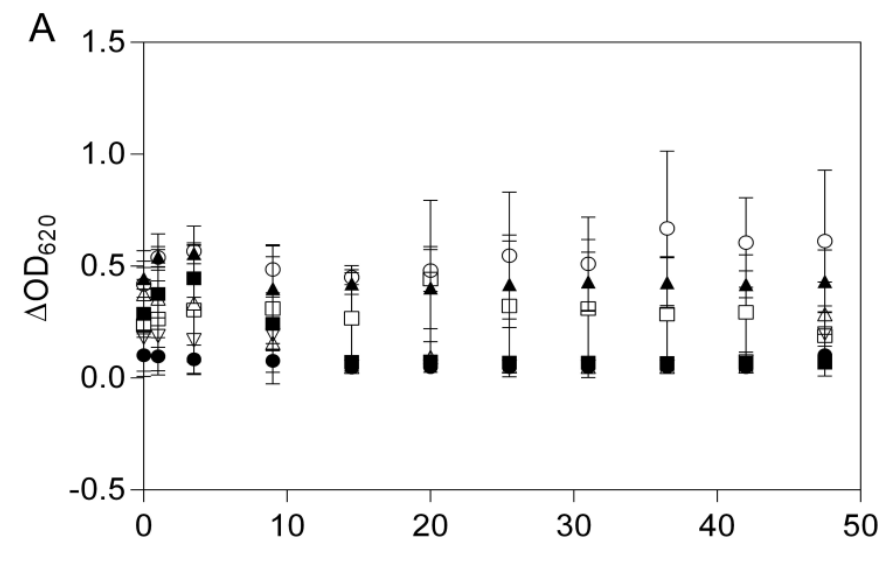

- pGAL4

- $\quad$ pGAL4.VP16

$\triangle \quad$ pGAL4.CiLBD.VP16

$\nabla \quad$ pGAL4.BsLBD.VP16

- pGAL4.CiLBDA31.VP16

- pGAL4.CiLBD

- pGAL4.BsLBD

[(-)-deoxydihydrokalafungin], $\mu \mathrm{M}$

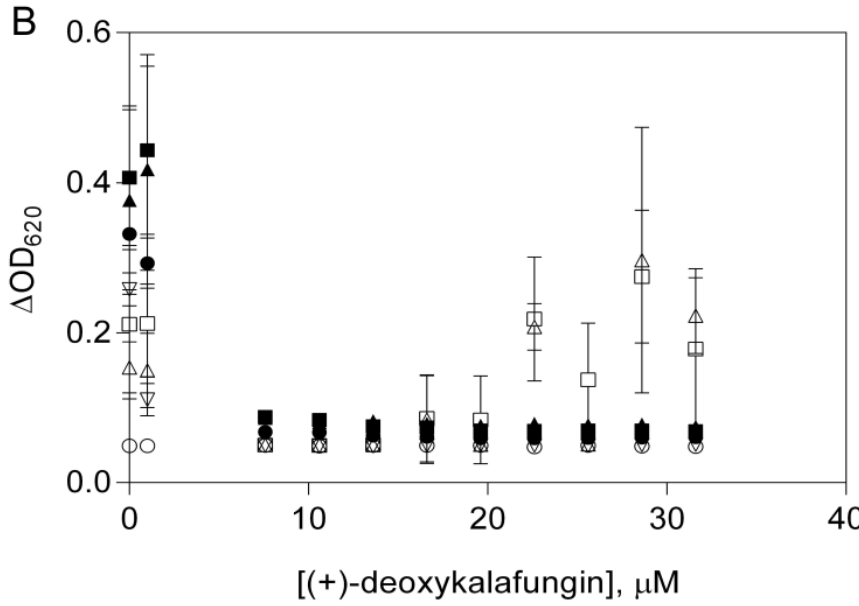

- pGAL4

- $\quad$ pGAL4.VP16

$\triangle \quad$ pGAL4.CiLBD

$\nabla \quad$ pGAL4.BsLBD

- pGAL4.CiLBDA31.VP16

- pGAL4.CiLBD.VP16

- pGAL4.BsLBD.VP16

Appendix 15 Suppression of growth of recombinant yeast strains by: (A) (-)deoxydihydrokalafungin and (B) (+)-deoxykalafungin. Cell densities were quantified by $\mathrm{OD}_{620}$ values. Growth suppression (i.e. toxic) effects were detected by changes in $O D_{620}$ values over time $\left(\Delta \mathrm{OD}_{620}=\mathrm{OD}_{620}\right.$ after $24 \mathrm{~h}-$ $\mathrm{OD}_{620}$ after $16 \mathrm{~h}$ ). Data points represent means of $\mathrm{n}=3$ replicates. Error bars show \pm one standard deviation. 


\section{APPENDIX SIXTEEN}

Appendix 16 Activation of the yeast bioassays by 13 emerging contaminants.

Ligand-dependent induction of $\beta$-galactosidase enzymatic activity was measured in yeast strains carrying plasmids pGAL4.CILBD431.VP16, pGAL4.CILBD.VP16 or pGAL4.BsLBD.VP16. Mean effective concentrations (EC 50 values) and 95\% confidence intervals $(95 \% \mathrm{Cl}$ ) are given in $\mu \mathrm{M}$. 95\% confidence intervals represent variation within a triplicate measurement $(\mathrm{n}$ = 3). Coefficients of variance (CV) are given in \% for triplicate intra-plate measurements. All compounds were dissolved in dimethyl sulfoxide (DMSO).

\begin{tabular}{|c|c|c|c|c|c|c|c|c|}
\hline \multirow[t]{2}{*}{ Chemical name } & \multirow[t]{2}{*}{ Toxin type } & \multirow{2}{*}{$\begin{array}{l}\text { Supplier } \\
\text { (Catalogue No.) }\end{array}$} & \multicolumn{2}{|c|}{ pGAL4.CILBD $\triangle 31 . V P 16$} & \multicolumn{2}{|c|}{ pGAL4.CiLBD.VP16 } & \multicolumn{2}{|c|}{ pGAL4.BsLBD.VP16 } \\
\hline & & & $\mathrm{EC}_{50}(95 \% \mathrm{Cl})$ & CV & $\mathrm{EC}_{50}(95 \% \mathrm{Cl})$ & CV & $\mathrm{EC}_{50}(95 \% \mathrm{Cl})$ & CV \\
\hline $\begin{array}{l}\text { Butylated } \\
\text { hydroxytoluene }\end{array}$ & Antioxidant & $\begin{array}{l}\text { Sigma-Aldrich } \\
\text { (B1378) }\end{array}$ & $7.1(2.3-22)$ & 37 & $7.2(3.1-17)$ & 41 & $\mathrm{NI}(\mathrm{DNC})$ & DNC \\
\hline $\begin{array}{l}\text { 4-chloro-3,5- } \\
\text { dimethylphenol }^{\text {b }}\end{array}$ & Antimicrobial & $\begin{array}{l}\text { Sigma-Aldrich } \\
(\mathrm{C}-4394)\end{array}$ & $15(4.8-43)$ & 39 & $61(37-98)$ & 41 & $29(16-50)$ & 25 \\
\hline 2-phenoxyethanol ${ }^{\mathrm{b}}$ & Antimicrobial & $\begin{array}{l}\text { Sigma-Aldrich } \\
(77699)\end{array}$ & $\mathrm{NI}(\mathrm{DNC})$ & DNC & $\mathrm{NI}(\mathrm{DNC})$ & DNC & $\mathrm{NI}(\mathrm{DNC})$ & DNC \\
\hline $\begin{array}{l}\text { Propyl-4-hydroxy- } \\
\text { benzoate }^{b}\end{array}$ & Antimicrobial & $\begin{array}{l}\text { Sigma-Aldrich } \\
\text { (P53357) }\end{array}$ & 237 (132-425) & 34 & 115 (DNC) & 39 & $\mathrm{NI}(\mathrm{DNC})$ & DNC \\
\hline
\end{tabular}


Appendix 16 (cont.) Activation of the yeast bioassays by 13 emerging contaminants.

\begin{tabular}{|c|c|c|c|c|c|c|c|c|}
\hline \multirow[t]{2}{*}{ Chemical name } & \multirow[t]{2}{*}{ Toxin type } & \multirow{2}{*}{$\begin{array}{l}\text { Supplier } \\
\text { (Catalogue No.) }\end{array}$} & \multicolumn{2}{|c|}{ pGAL4.CILBD $\triangle 31 . V P 16$} & \multicolumn{2}{|c|}{ pGAL4.CiLBD.VP16 } & \multicolumn{2}{|c|}{ pGAL4.BsLBD.VP16 } \\
\hline & & & $\mathrm{EC}_{50}(95 \% \mathrm{Cl})$ & CV & $\mathrm{EC}_{50}(95 \% \mathrm{Cl})$ & CV & $\mathrm{EC}_{50}(95 \% \mathrm{Cl})$ & CV \\
\hline $\begin{array}{l}\text { 4-methyl-benzylidene } \\
\text { camphor }^{\text {b }}\end{array}$ & EDC & $\begin{array}{l}\text { Sigma-Aldrich } \\
\text { (78551) }\end{array}$ & $\mathrm{NI}(\mathrm{DNC})$ & DNC & $101(78-131)$ & 34 & $103(75-141)$ & 36 \\
\hline $\begin{array}{l}\text { Octyl methoxy- } \\
\text { cinnamate }^{b}\end{array}$ & EDC & $\begin{array}{l}\text { Sigma-Aldrich } \\
\text { (78848) }\end{array}$ & $37(20-67)$ & 17 & $44(13-151)$ & 10 & $377(2.4-60,283)$ & 123 \\
\hline Triclosan $^{a}$ & EDC & $\begin{array}{l}\text { Sigma-Aldrich } \\
\text { (72779) }\end{array}$ & $\mathrm{NI}(\mathrm{DNC})$ & DNC & $\mathrm{NI}(\mathrm{DNC})$ & DNC & $\mathrm{NI}(\mathrm{DNC})$ & DNC \\
\hline Benzophenone $^{\mathrm{b}}$ & EDC & $\begin{array}{l}\text { Sigma-Aldrich } \\
\text { (B9300) }\end{array}$ & 382 (DNC) & DNC & $348(312-388)$ & 23 & $\mathrm{NI}(\mathrm{DNC})$ & DNC \\
\hline Ketoconazole ${ }^{a}$ & Antifungal & $\begin{array}{l}\text { EMD Chemicals } \\
(420600)\end{array}$ & $\sim 596(\mathrm{DNC})^{\mathrm{c}}$ & DNC & $509(455-570)^{\mathrm{c}}$ & 13 & $582(391-865)^{\mathrm{c}}$ & 18 \\
\hline 2-phenylphenol ${ }^{\mathrm{b}}$ & Antifungal & $\begin{array}{l}\text { Acros Organics } \\
(130760050)\end{array}$ & $\mathrm{NI}(\mathrm{DNC})$ & DNC & $\mathrm{NI}(\mathrm{DNC})$ & DNC & $\mathrm{NI}(\mathrm{DNC})$ & DNC \\
\hline Radicicol $^{\mathrm{b}}$ & Antifungal & $\begin{array}{l}\text { Cayman Chemical } \\
(13089)\end{array}$ & $\mathrm{NI}(\mathrm{DNC})$ & DNC & $\mathrm{NI}(\mathrm{DNC})$ & DNC & $\mathrm{NI}(\mathrm{DNC})$ & DNC \\
\hline $\mathrm{DEET}^{\mathrm{b}}$ & Insecticide & $\begin{array}{l}\text { Fluka } \\
\text { (36542) }\end{array}$ & $\mathrm{NI}(\mathrm{DNC})$ & DNC & $\mathrm{NI}(\mathrm{DNC})$ & DNC & $\mathrm{NI}(\mathrm{DNC})$ & DNC \\
\hline
\end{tabular}


Appendix 16 (cont.) Activation of the yeast bioassays by 13 emerging contaminants.

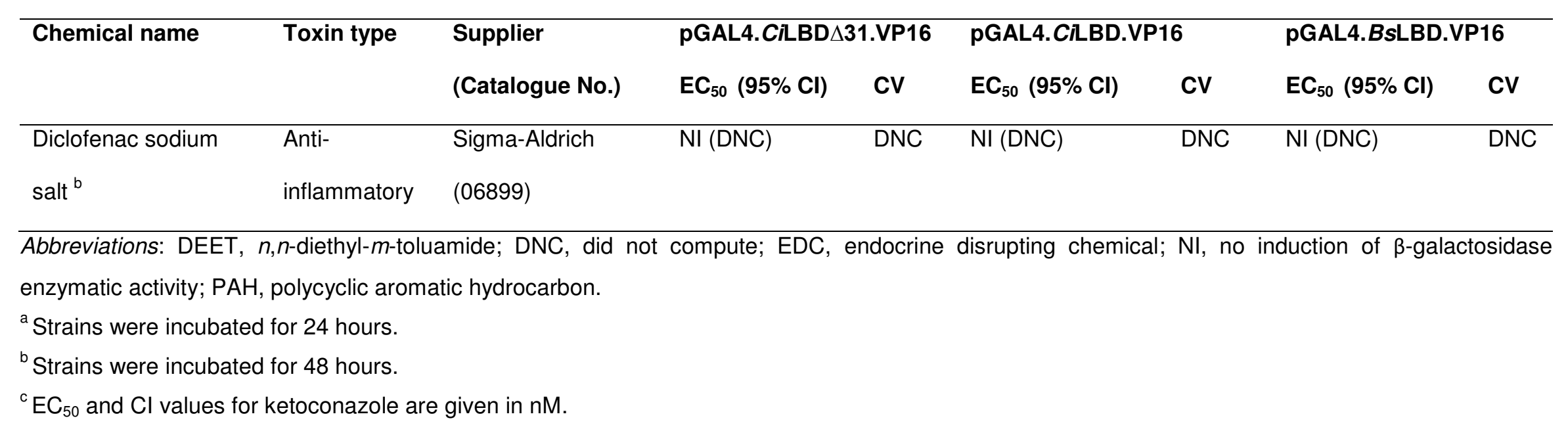




\section{APPENDIX SEVENTEEN}

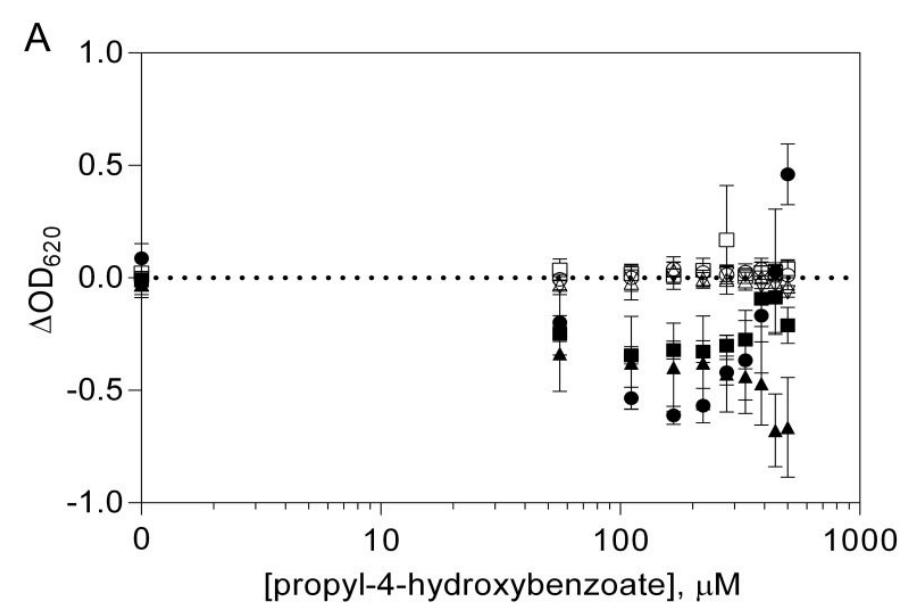

- pGAL4

- $\quad$ PGAL4.VP16

$\triangle \quad$ pGAL4.CiLBD

$\nabla \quad$ pGAL4.BsLBD

- pGAL4.CiLBD $\triangle 31 . V P 16$

- pGAL4.CiLBD.VP16

- pGAL4.BsLBD.VP16

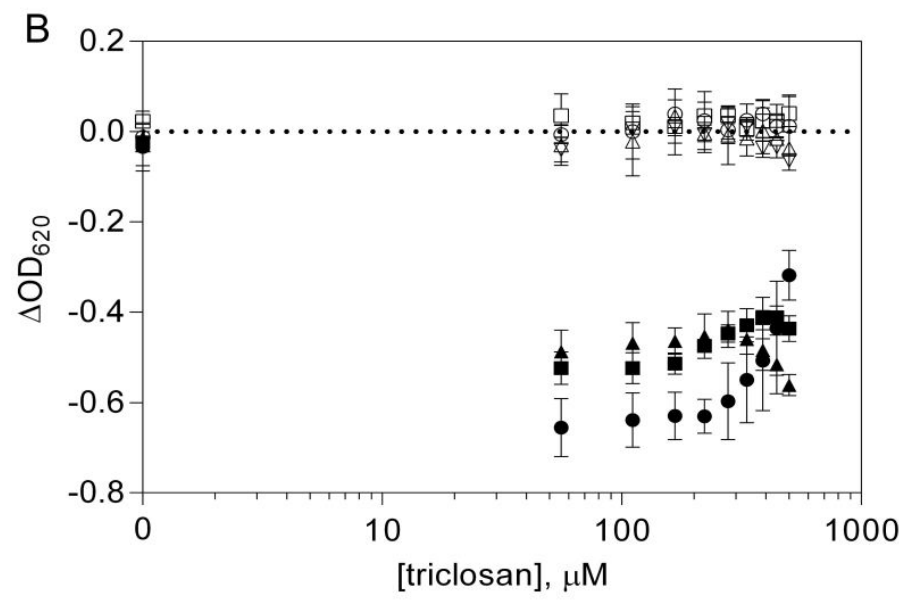

- pGAL4

- $\quad$ pGAL4.VP16

$\triangle \quad$ pGAL4.CiLBD

$\nabla \quad$ pGAL4.BsLBD

- pGAL4.CiLBD $\triangle 31 . V P 16$

- pGAL4.CiLBD.VP16

- pGAL4.BsLBD.VP16

Appendix 17 Suppression of growth of recombinant yeast strains by: (A) propyl-4-hydroxybenzoate and (B) triclosan. Cell densities were quantified by $\mathrm{OD}_{620}$ values. Growth suppression (i.e. toxic) effects were detected by changes in $\mathrm{OD}_{620}$ values over time $\left(\Delta O \mathrm{D}_{620}=\mathrm{OD}_{620}\right.$ after $48 \mathrm{~h}-\mathrm{OD}_{620}$ after $\left.0 \mathrm{~h}\right)$. Data points represent means of $n=3$ replicates. Error bars show \pm one standard deviation. Dotted lines indicate baseline. 


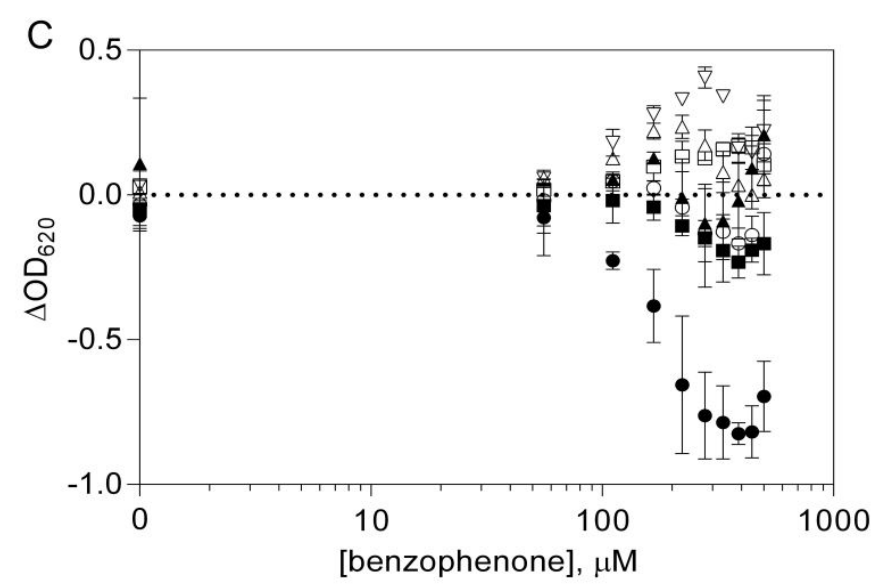

- pGAL4

- $\mathrm{pGAL4.VP16}$

$\triangle$ pGAL4.CiLBD

$\nabla \quad$ pGAL4.BsLBD

- pGAL4.CiLBDA31.VP16

- pGAL4.CilBD.VP16

- pGAL4.BsLBD.VP16

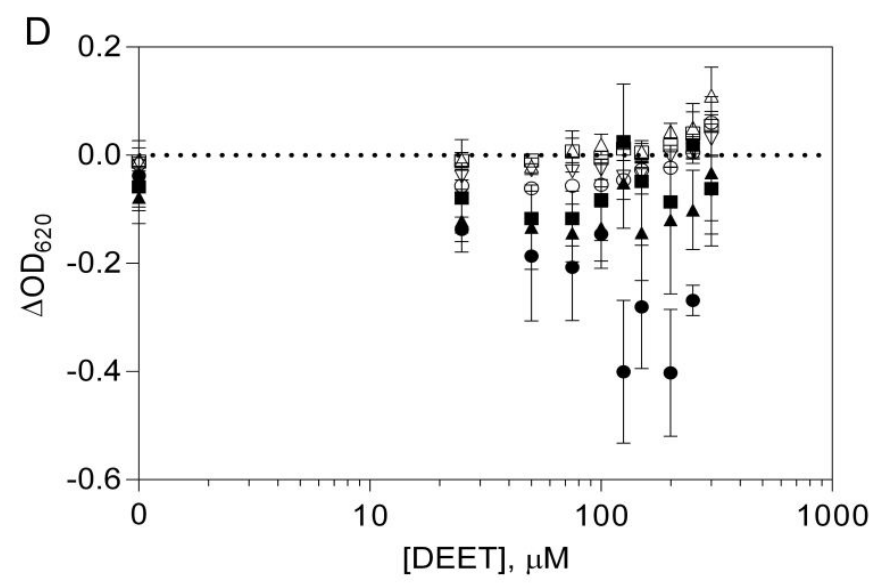

- pGAL4

口 $\quad$ PGAL4.VP16

$\triangle \quad$ pGAL4.CiLBD

$\nabla \quad$ pGAL4.BSLBD

- pGAL4.CiLBD $\Delta 31 . V P 16$

- pGAL4.CiLBD.VP16

- pGAL4.BsLBD.VP16

Appendix 17 (cont.) Suppression of growth of recombinant yeast strains by: (C) benzophenone and (D) $n, n$-diethyl- $m$-toluamide (DEET). Cell densities were quantified by $\mathrm{OD}_{620}$ values. Growth suppression (i.e. toxic) effects were detected by changes in $\mathrm{OD}_{620}$ values over time $\left(\Delta \mathrm{OD}_{620}=\mathrm{OD}_{620}\right.$ after $48 \mathrm{~h}-$ $\mathrm{OD}_{620}$ after $0 \mathrm{~h}$ ). Data points represent means of $\mathrm{n}=3$ replicates. Error bars show \pm one standard deviation. Dotted lines indicate baseline. 


\section{APPENDIX EIGHTEEN}

\section{Presentations at conferences and meetings:}

Richter I \& Fidler AE (2014) Tunicate xenobiotic receptors: application in highthroughput bioassays for bioactive compounds. Satellite Meeting Frontiers of Biology, Wellington, New Zealand.

Richter I \& Fidler AE (2014) Tunicate xenobiotic-activated nuclear receptors: their application as sensor elements in high-throughput bioassays for marine microalgal biotoxins. International Conference on Harmful Algae, Wellington, New Zealand.

Richter I \& Fidler AE (2014) Tunicate xenobiotic activated nuclear receptors: their application as sensor elements in high-throughput bioassays for marine microalgal biotoxins. New Zealand Marine Science Annual Conference, Nelson, New Zealand. Award for 'Best talk relevant to the Seafood Industry'.

Richter I \& Fidler AE (2014) Sea squirts in bioassays- a tool to detect harmful marine biotoxins. A Conference for Women in Science, Wellington, New Zealand.

Richter I \& Fidler AE (2013) Functionally significant polymorphisms in tunicate xenobiotic receptors. International Conference of Chemical Ecology, Melbourne, Australia.

Richter I \& Fidler AE (2012) Expression and genetic diversity of tunicate xenobiotic receptors. Next-Generation Sequencing Conference, Dunedin, New Zealand.

Richter I \& Fidler AE (2012) Expression and genetic diversity of tunicate xenobiotic receptors. Australian Centre for Evolutionary Biology and Biodiversity, University of Adelaide, Australia. 


\section{APPENDIX NINETEEN}

\section{Publications in refereed journals:}

Richter I \& Fidler AE (2014) Marine invertebrate xenobiotic-activated nuclear receptors: their application as sensor elements in high-throughput bioassays for marine bioactive compounds. Marine Drugs. 12(11): 5590618. doi: $10.3390 / \mathrm{md} 12115590$.

Richter, I \& Fidler AE (2015) Detection of marine microalgal biotoxins using bioassays based on functional expression of tunicate xenobiotic receptors in yeast. Toxicon 95(0): 13-22. doi: 10.1016/j.toxicon.2014.12.011. 


\title{
OPEN ACCESS \\ marine drugs \\ ISSN 1660-3397 \\ www.mdpi.com/joumal/marinedrugs
}

Review

\section{Marine Invertebrate Xenobiotic-Activated Nuclear Receptors: Their Application as Sensor Elements in High-Throughput Bioassays for Marine Bioactive Compounds}

Ingrid Richter ${ }^{1,2, *}$ and Andrew E. Fidler ${ }^{1,3,4}$

1 Environmental Technology Group, Cawthron Institute, Private Bag 2, Nelson 7012, New Zealand; E-Mail: andrew.fidler@cawthron.orgnz

2 School of Biological Science, Victoria University of Wellington, P.O. Box 600, Wellington 6140, New Zealand

3 Maurice Wilkins Centre for Molecular Biodiscovery, University of Auckland, Auckland 1142, New Zealand

4 Institute of Marine Science, University of Auckland, Auckland 1142, New Zealand

* Author to whom correspondence should be addressed; E-Mail: ingrid richter@ cawthron.orgnz; Tel.: +64-3-548-2319; Fax: +64-3-546-9464.

Extemal Editor: Paul Long

Received: 20 May 2014; in revised form: 31 October 2014 / Accepted: 11 November 2014 /

Published: 24 November 2014

\begin{abstract}
Developing high-throughput assays to screen marine extracts for bioactive compounds presents both conceptual and technical challenges. One major challenge is to develop assays that have well-grounded ecological and evolutionary rationales. In this review we propose that a specific group of ligand-activated transcription factors are particularly well-suited to act as sensors in such bioassays. More specifically, xenobiotic-activated nuclear receptors (XANRs) regulate transcription of genes involved in xenobiotic detoxification. XANR ligand-binding domains (LBDs) may adaptively evolve to bind those bioactive, and potentially toxic, compounds to which organisms are normally exposed to through their specific diets. A brief overview of the function and taxonomic distribution of both vertebrate and invertebrate XANRs is first provided. Proof-of-concept experiments are then described which confirm that a filter-feeding marine invertebrate XANR LBD is activated by marine bioactive compounds. We speculate that increasing access to marine invertebrate genome sequence data, in combination with the expression of functional recombinant marine invertebrate XANR LBDs, will facilitate the generation of
\end{abstract}




\section{Detection of marine microalgal biotoxins using bioassays based on functional expression of tunicate xenobiotic receptors in yeast}

Ingrid Richter ${ }^{a, b}{ }^{*}$, Andrew E. Fidler ${ }^{2, c, d}$

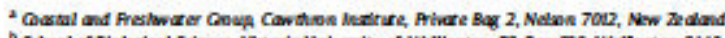

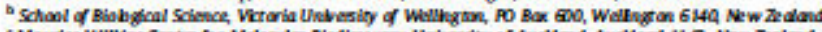

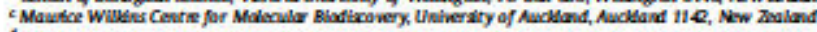

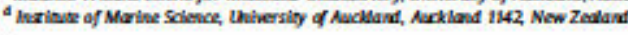

\section{A R TICLE IN FO}

Nvacke hizary

Secolved 21 cerober 2014

Mecolved in revised

22 December 2004

hocepsed 27 Decernber 2014

27 pecenter 2014

Neymonts:

China intsarsids

Bosylas scthasent

Pregnane X receptor

Succharamyces aseretis

Marine bicrodins

Biovasuy

\begin{abstract}
A B S T R ACT
Marine microal ge can produce biotoxins that cause widesprexd poisoning in marine ecosystems and may also affect human health. While established microalgal biotoxins are detectable using chemica methods, a need remains for robust, inexpensive biassays. ligand binding domains (LBDs) from a turicat nudear receptor, VDR/PXRn, which is arthalogous to both the vertebrate pregrane $\mathrm{X}$ receptor (PXR) and the vitamin D reasptar (VDR), can be activated by microalgal hiotoxins when oxpressed in mammalian all lines Building on this o berervation, we developed a gereric recombinant yeast biassay platform that expresses chimeric proteins containing turicat VDR/PXRa LBDs which medise ligand. dependent transcription of a reporter gene (lacZ) enoding an essily zsayed enzyme ( $\beta$-galatosi.

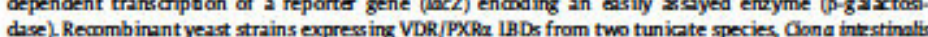

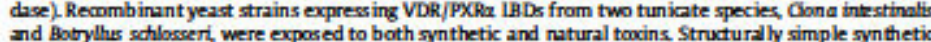

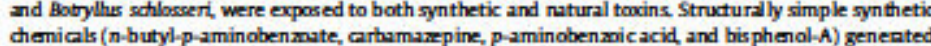

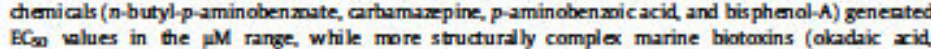
pecteno toxin-11, and portimine) activated the $x$ says in the $n M$ range. Given the large number of turicats species we propose that tunicate VDR/PXR IBDs may be used as 'sensar elements' in similar year t based high-throughtput bio assays for detection of established microalgal bio twoins and uncharacterised marine biasctive compounds.
\end{abstract}

Q 2014 Elsevier Let. All rights reserved.

\section{Introduction}

Some marine microalgal taxa can produce highly toxic chemicals that, on occasion, cause widespread poisoning within mar ine

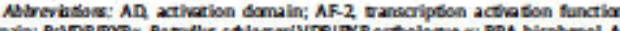

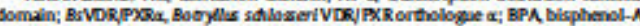

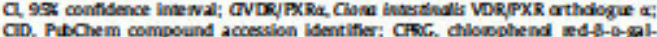

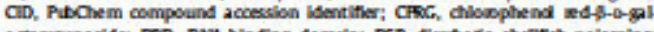

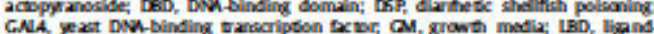
CNA, yext DNA binding trasciptipton bCwar, CM, growth medix; LBD, llgand binding Sama in; MCS, mukiple doning slix; MM minimal meflix; NQ nodear re.

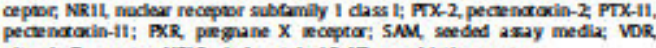

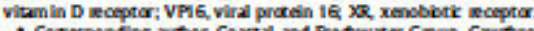

Copron Inestruse, Privale Bay 2, Netson 012 New Zealand

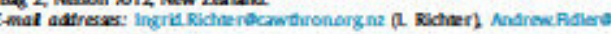
awthonagraz (AE Fiaer)

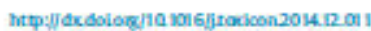

0041-0 DH/ 2014 Baevier Lat Nil righrs reservel ecosystems and which may also affect human health, particularly when accumulated in filter-feeding shellfish onsumed by humans (Mackenz ie et al . 2011: Wang, 2008; Wear and Gardner, 2001: Zhang et at, 2013). Over the past two decades internationally accepted methodologies for the detection of such natural marine biotoxins have become increasingly based on chemical, rather than biological assays (Gerssen et al__2010; McNabbet al_ 2012; Suzuk and Quilliam, 2011). While such chemistry-based detection methods are generally reliable and highly specific, they require specialised equipment and skills along with detailed structural knowledge regarding the targeted biotoxins, and so do not allow detection of unknown marine biotoxins (Humpage et al, 2010; Nicolas et al, 2014). Although a range of in vitro bioassays for microalgal biotoxins have been developed (Banerjee et aL, 2013; Bovee et al, 2011; Niolas et al, 2014), currently the most widelyused microalgal biobxin bioassyy remains the unreliatie and ethically questionable mouse mortality bioassry (Botana et al. 2009, Buckland, 2010; Stewart and McLeod, 2014). Consequently. 
